\title{
Classification, geometry and applications of supersymmetric backgrounds
}

\author{
U. Gran ${ }^{\text {a }}$, J. Gutowski ${ }^{\mathrm{b}}$, G. Papadopoulos ${ }^{\mathrm{c}, *, 1}$ \\ ${ }^{a}$ Department of Physics, Division for Theoretical Physics, Chalmers University of Technology, SE-412 96 Göteborg, Sweden \\ ${ }^{\mathrm{b}}$ Department of Mathematics, University of Surrey, Guildford, GU2 $7 \mathrm{XH}, \mathrm{UK}$ \\ ' Department of Theoretical Physics, CERN, 1211 Geneva 23, Switzerland
}

\section{A R T I C L E I N F O}

\section{Article history:}

Received 10 October 2018

Accepted 20 November 2018

Available online $\mathrm{xxxx}$

Editor: S. Stieberger

\begin{abstract}
A B S T R A C T
We review the remarkable progress that has been made the last 15 years towards the classification of supersymmetric solutions with emphasis on the description of the bilinears and spinorial geometry methods. We describe in detail the geometry of backgrounds of key supergravity theories, which have applications in the context of black holes, string theory, M-theory and the AdS/CFT correspondence unveiling a plethora of existence and uniqueness theorems. Some other aspects of supersymmetric solutions like the Killing superalgebras and the homogeneity theorem are also presented, and the non-existence theorem for certain smooth supergravity flux compactifications is outlined. Amongst the applications described is the proof of the emergence of conformal symmetry near black hole horizons and the classification of warped AdS backgrounds that preserve more than 16 supersymmetries.
\end{abstract}

(C) 2018 Elsevier B.V. All rights reserved.

\section{Contents}

1. Introduction.

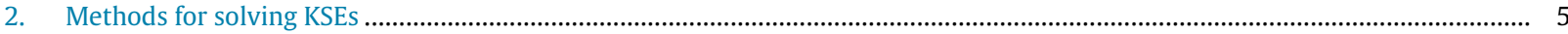

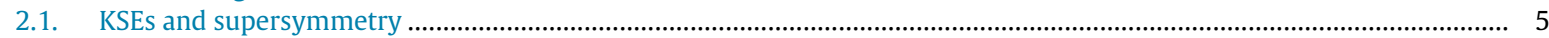

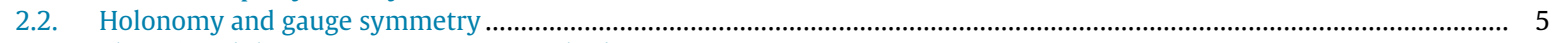

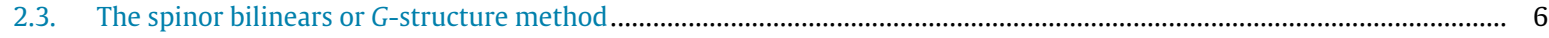

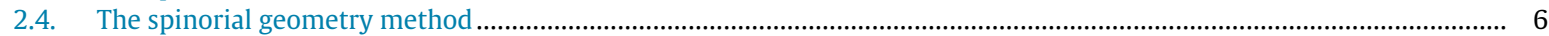

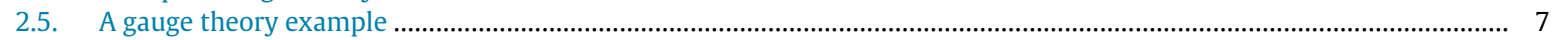

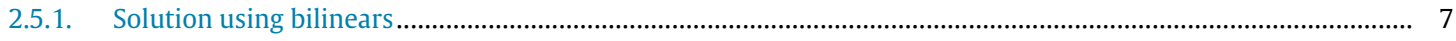

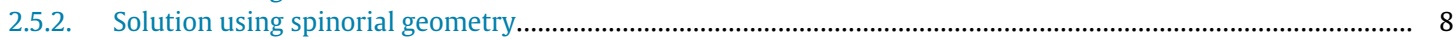

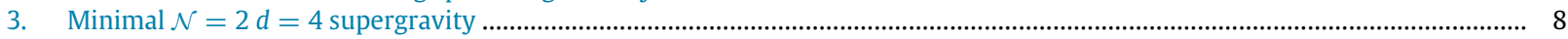

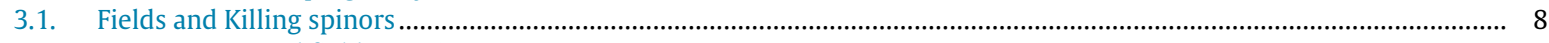

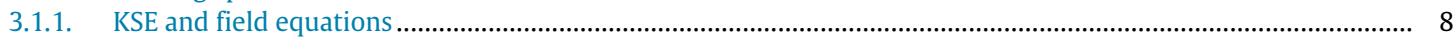

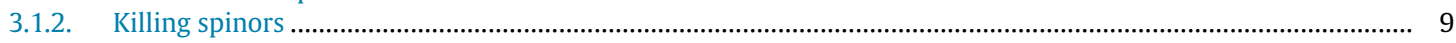

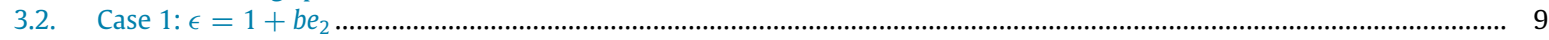

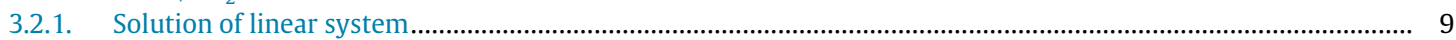

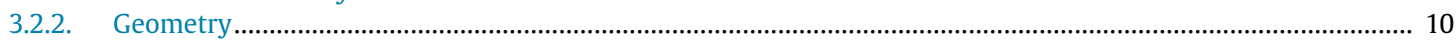

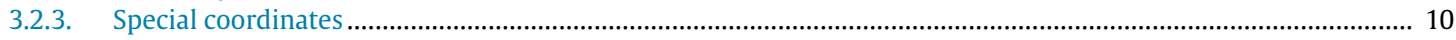

* Corresponding author.

E-mail address: george.papadopoulos@kcl.ac.uk (G. Papadopoulos).

1 On study leave: Department of Mathematics, King's College London, Strand, London WC2R 2LS, UK. 
3.2.4. Solutions.

3.3. Case $2: \epsilon=1+a e_{1}$

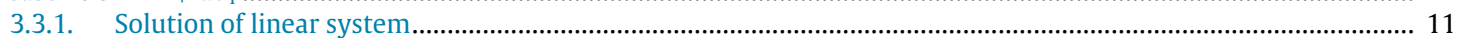

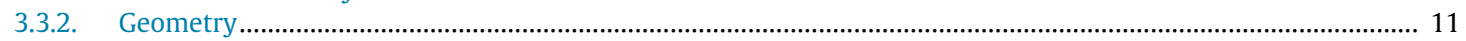

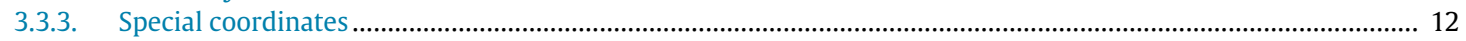

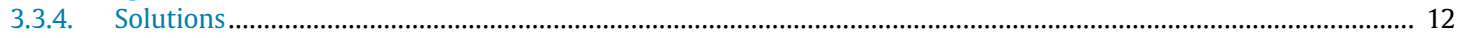

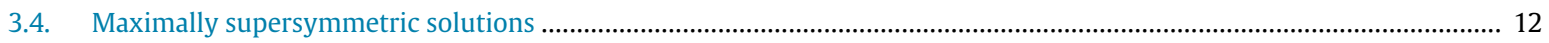

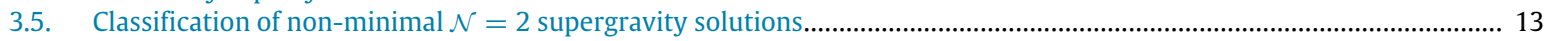

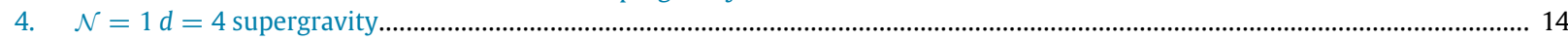

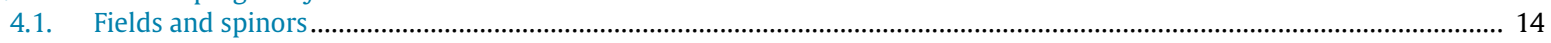

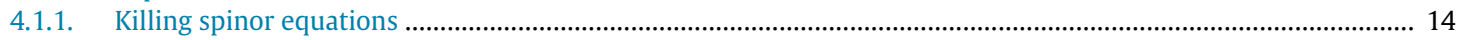

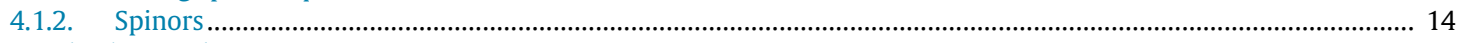

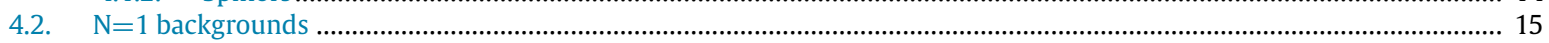

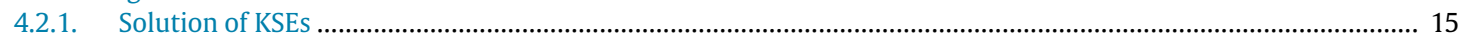

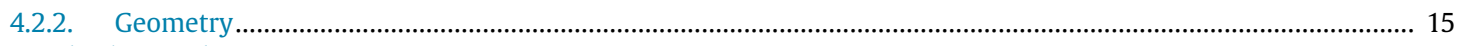

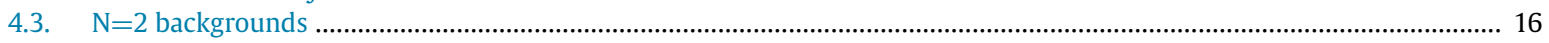

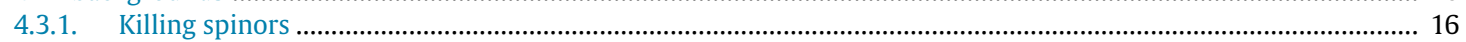

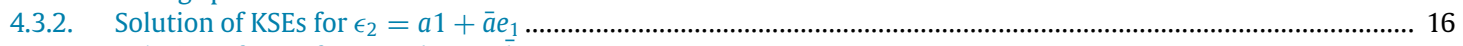

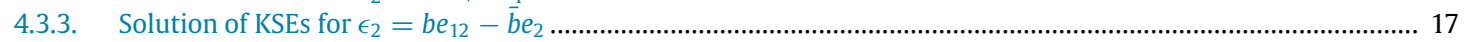

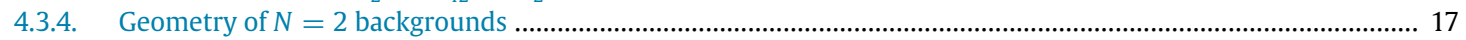

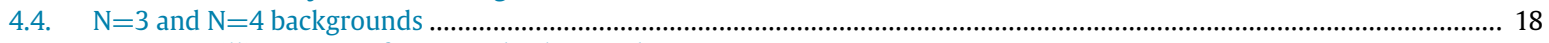

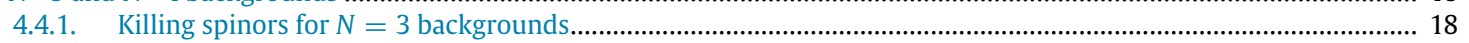

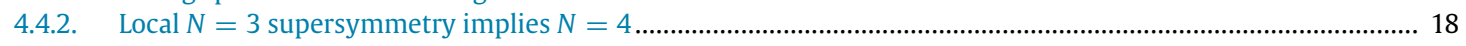

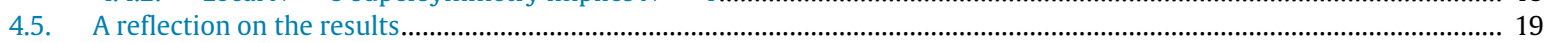

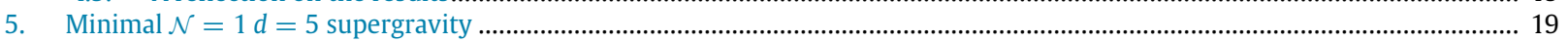

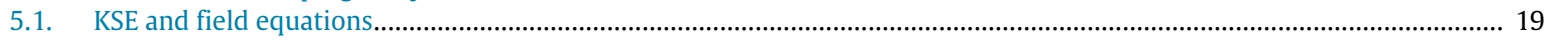

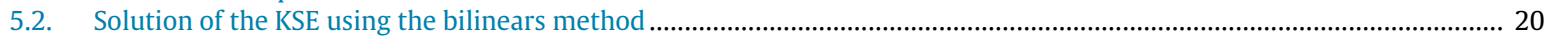

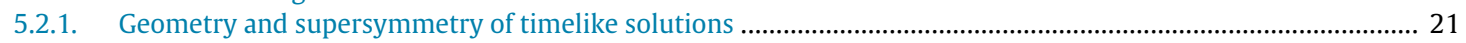

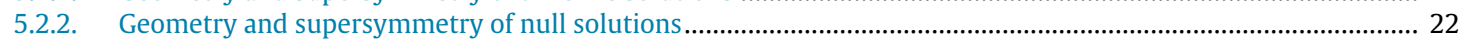

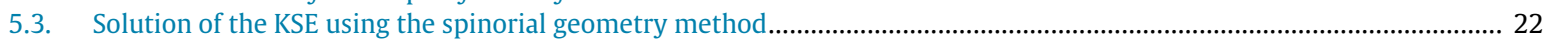

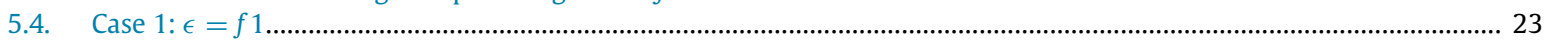

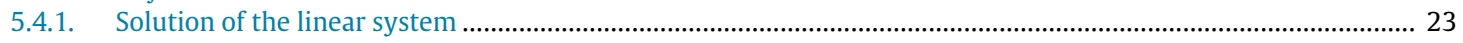

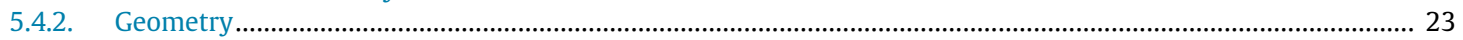

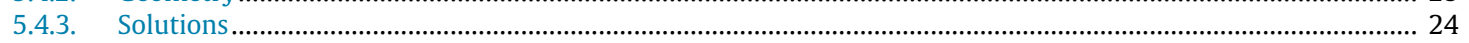

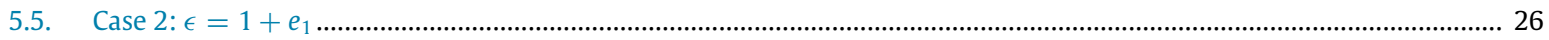

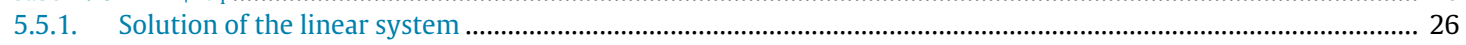

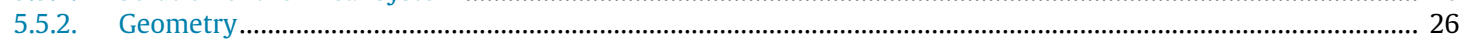

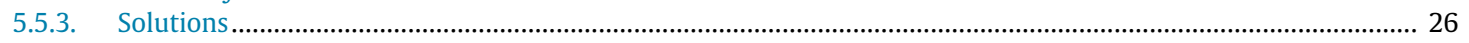

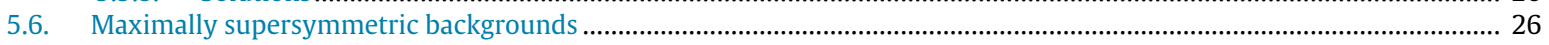

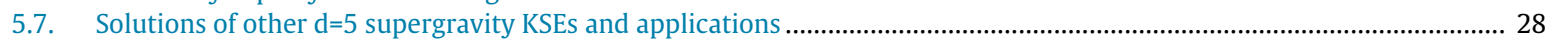

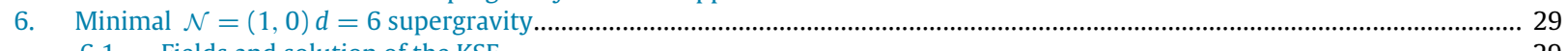

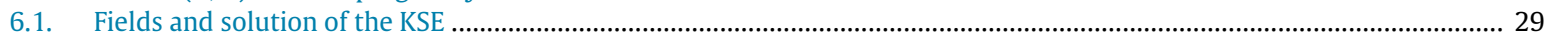

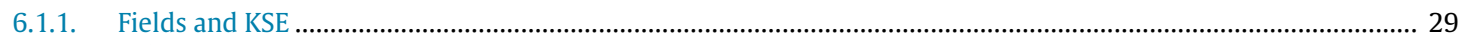

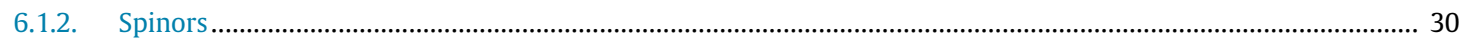

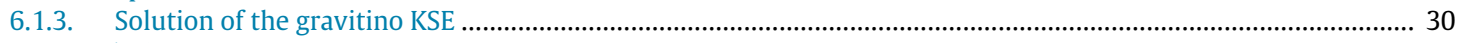

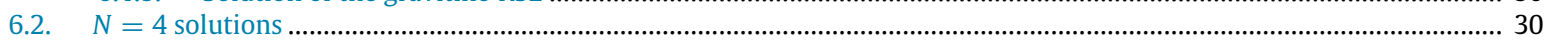

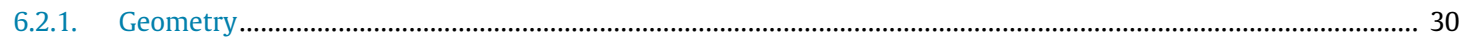

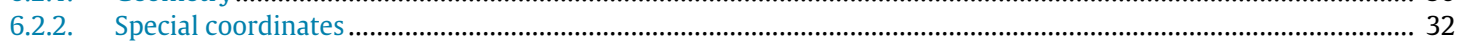

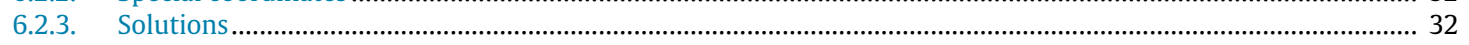

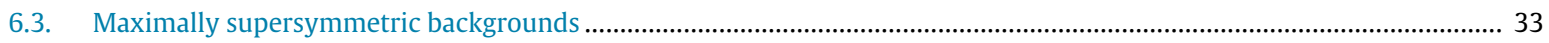

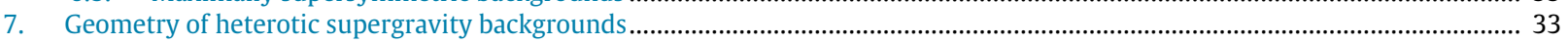

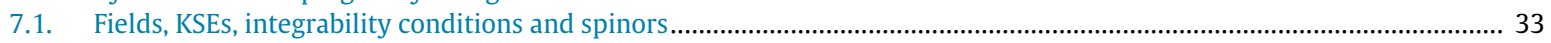

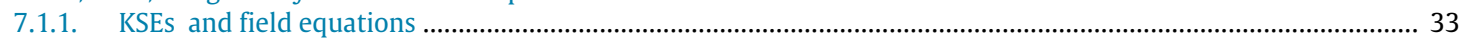

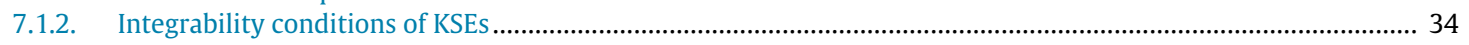

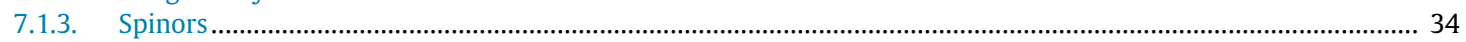

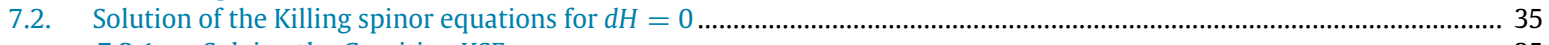

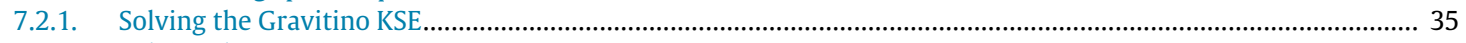

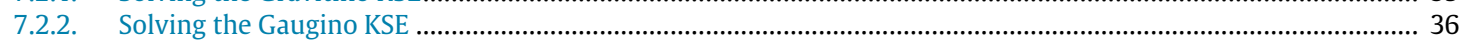

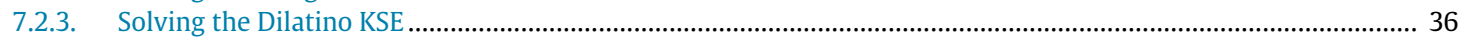

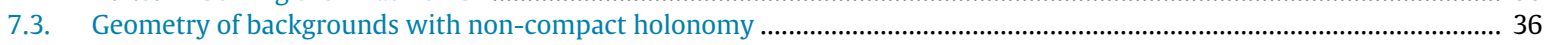

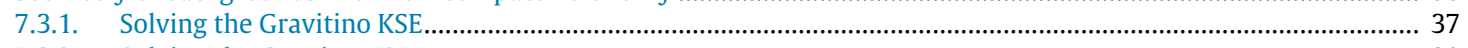

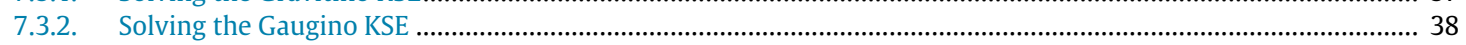

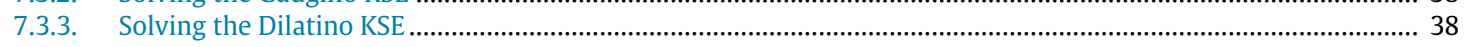

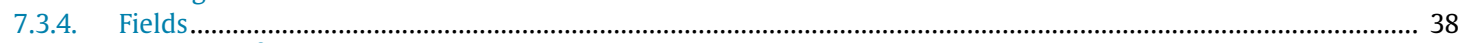

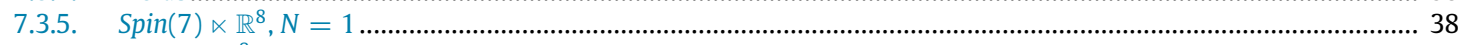

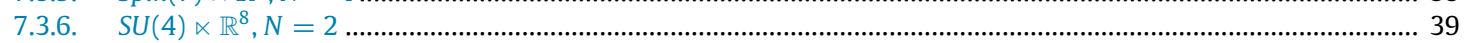




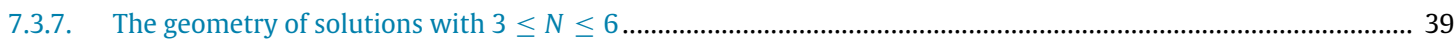

7.3.8. $\quad \mathbb{R}^{8}, N=8$

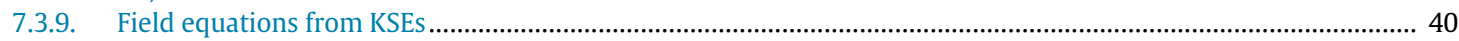

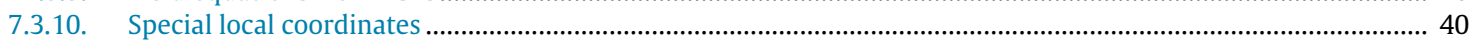

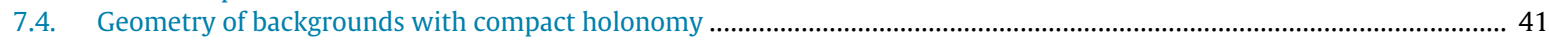

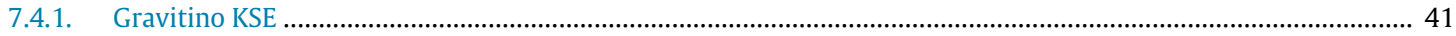

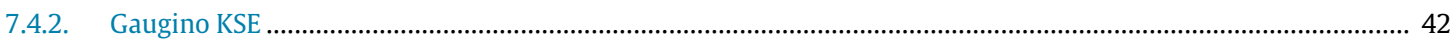

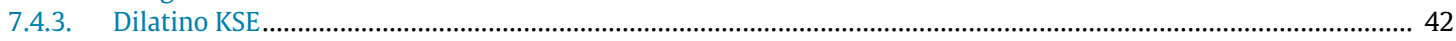

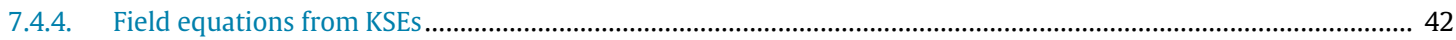

7.4.5. $\quad G_{2}, N=2$

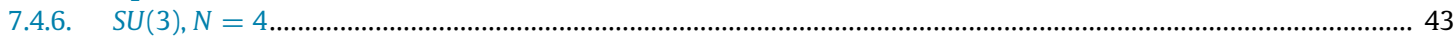

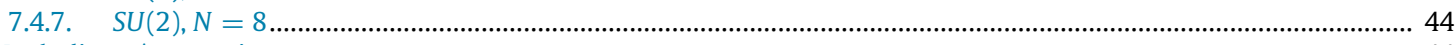

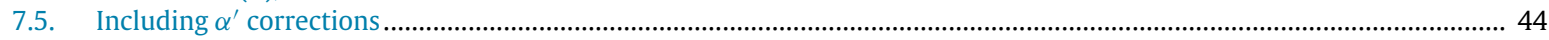

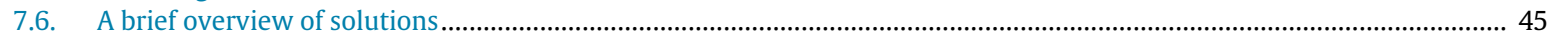

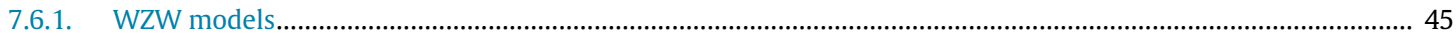

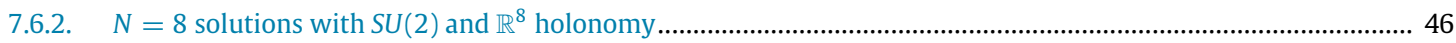

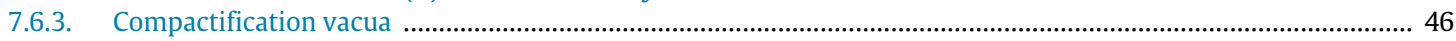

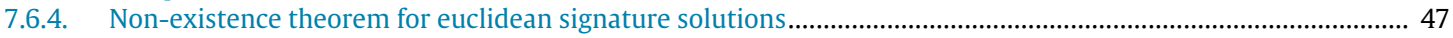

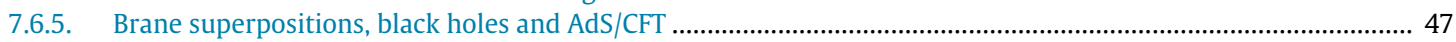

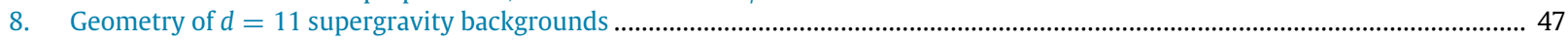

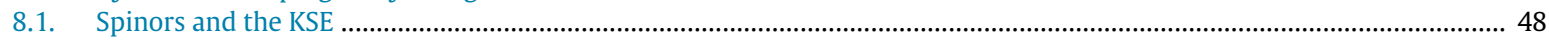

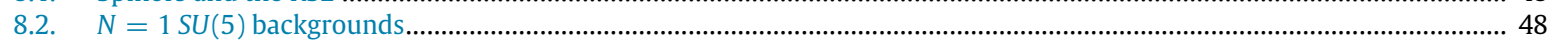

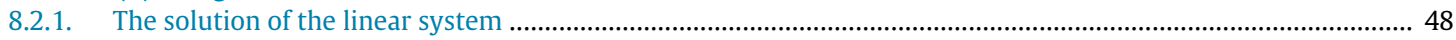

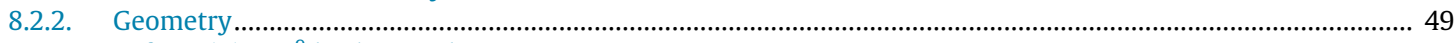

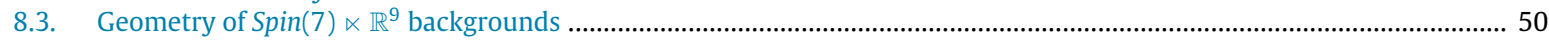

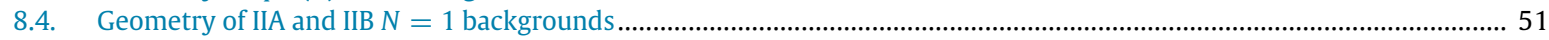

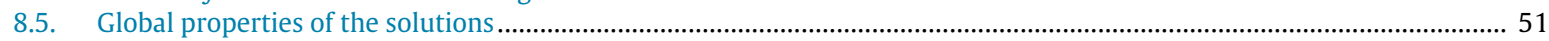

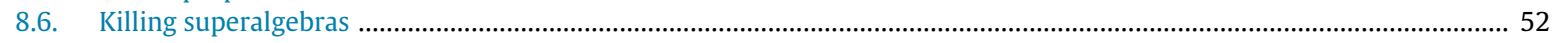

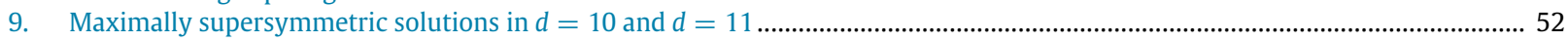

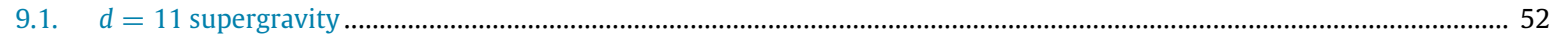

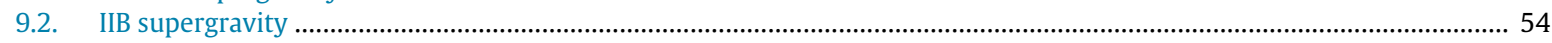

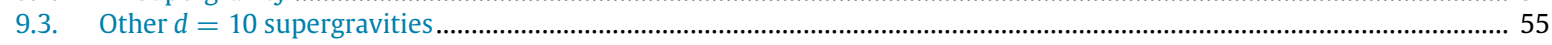

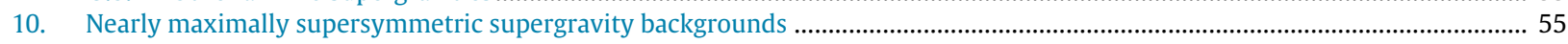

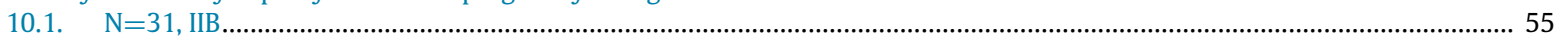

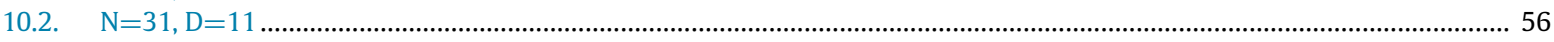

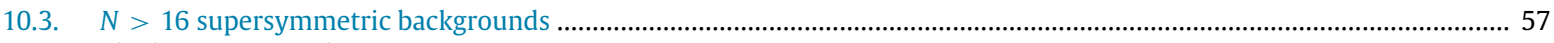

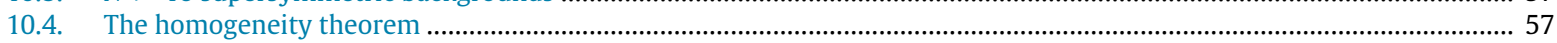

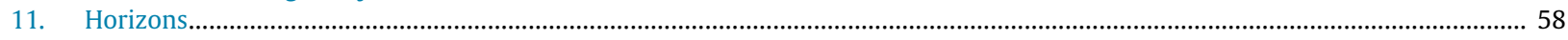

11.1. Symmetry enhancement near black hole and brane horizons................................................................................. 58

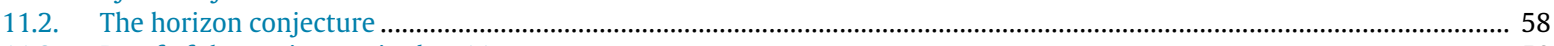

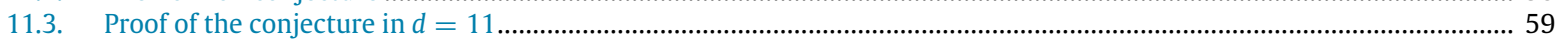

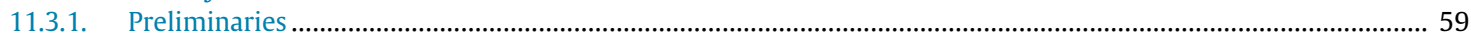

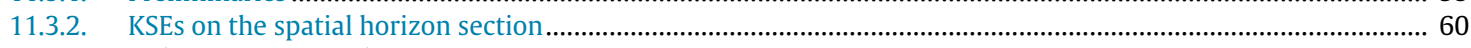

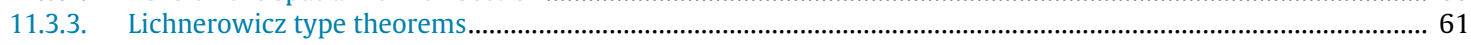

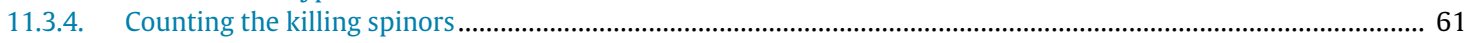

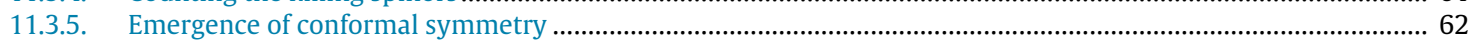

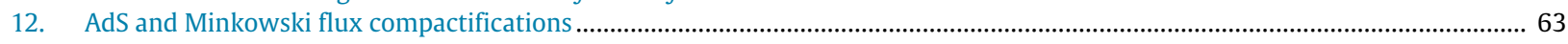

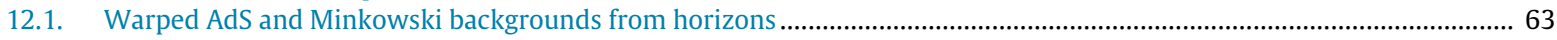

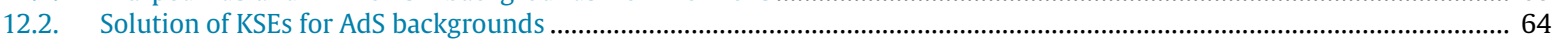

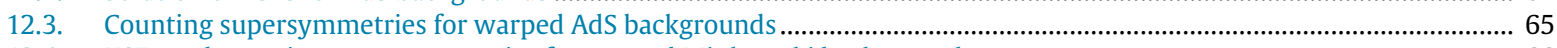

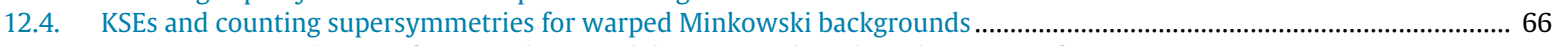

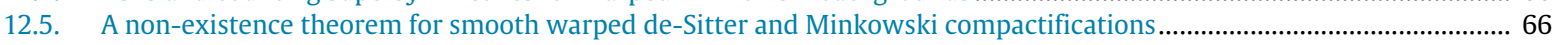

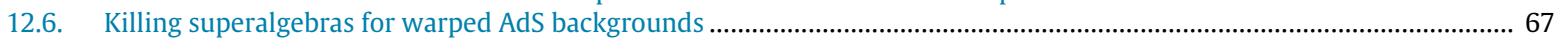

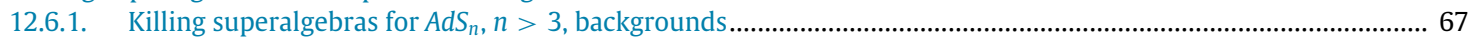

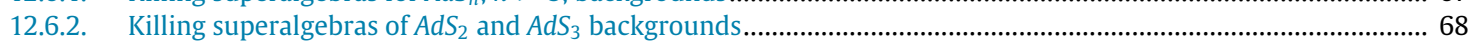

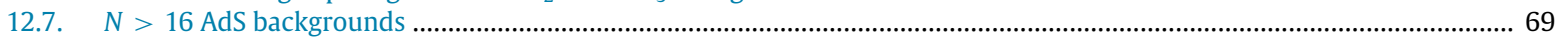

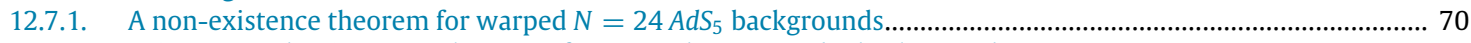

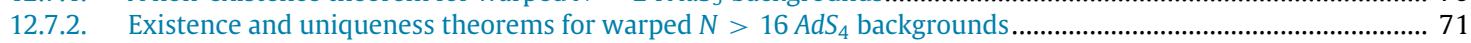

13. Conclusions.

Acknowledgements

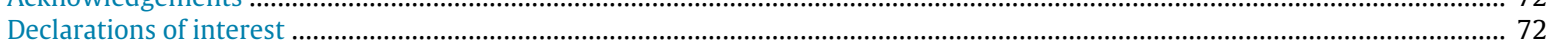

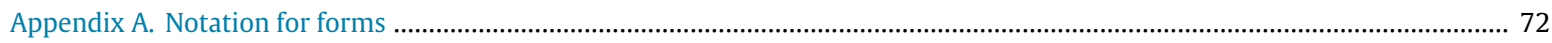

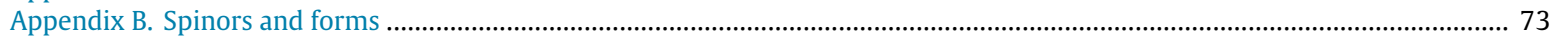

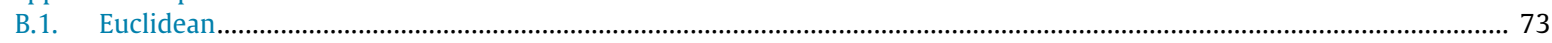

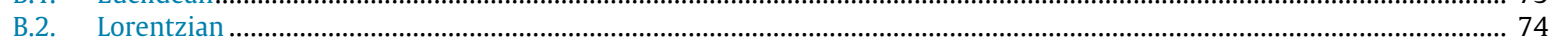

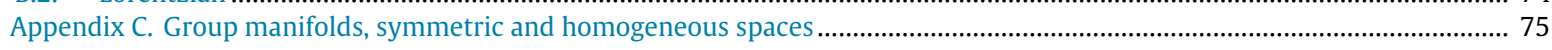


C.1. Homogeneous spaces
C.2. Cahen-Wallach spaces...
Appendix D. Fierz identities for $d=5$ supergravity
Appendix E. $d=11$ and type II $d=10$ supergravities.
E.1. $\quad d=11$ supergravity
E.2. $\quad$ IIB supergravity
References

\section{Introduction}

General relativity has brought a momentous change in the relationship between physics and geometry as the gravitational force at large scales is modelled in terms of geometry. The relevant geometry involved is that of manifolds equipped with a Lorentzian signature metric. The interplay between general relativity and manifold theory has led to the rapid development of both fields. The investigation of solutions of general relativity has had a profound impact on our understanding of the universe and the matter it contains. It has led to the introduction of black holes, the discovery of gravitational waves and to cosmological models which describe the evolution of our universe.

Following the general relativity paradigm, supersymmetric systems, which include string theory and M-theory, admit a new class of solutions, the "supersymmetric solutions", which in addition to the field equations also solve the Killing spinor equations (KSEs). These arise from the vanishing condition of the supersymmetry variations of the fermions of these theories. It was soon realized that such solutions may saturate certain Bogomol'nyi type bounds, and because of this they are also called "BPS" solutions. In gauge theories supersymmetric solutions include monopoles and instantons which play a central role in the understanding of strong coupling dynamics and non-perturbative corrections to these theories. In gravitational theories supersymmetric solutions include extreme black hole solutions for Einstein-Maxwell type theories, as well as gravitational waves. In string theory and M-theory supersymmetric solutions include compactification vacua, extreme black holes and brane solutions. The latter are considered as the solitons of these theories and they have played a central role in unravelling the string dualities and in the microstate counting of black hole entropy, see [1-3]. These applications have continued in the context of the AdS/CFT correspondence [4] as the gravitational backgrounds which correspond to the vacuum states of dual superconformal theories are supersymmetric. There is a plethora of supersymmetric solutions and the research is ongoing as they have widespread applications, for reviews see e.g. [5,6]. The first systematic investigation of supersymmetric solutions was done by Tod who used twistorial techniques to solve the KSE of minimal $\mathcal{N}=2 d=4$ supergravity and classify all such solutions [7].

The purpose of this review is to summarize the significant progress that has been made the last 15 years in classifying the supersymmetric backgrounds. The problem has been solved for a large number of supergravity theories and the aim is to present the development and produce a guide to the field. The focus will be to explain the methods that have been used for this as well as to describe some of the key results that have been obtained. These include insights into the structure of all supersymmetric solutions in some theories, and the proof of existence and uniqueness theorems for several classes of solutions. Other aspects of the supersymmetric solutions, like their Killing superalgebras, the homogeneity theorem and a non-existence theorem for de-Sitter and Minkowski supergravity flux compactifications, are also included. The classification of maximal and near maximal supersymmetric backgrounds of some supergravity theories is also described. Applications of the results will also be considered in the context of black holes, string theory, M-theory and the AdS/CFT correspondence. Some aspects of the fascinating connection between supersymmetric backgrounds and special geometric structures will be presented. A generalization of classic results like the Lichnerowicz theorem will also be given as part of the proof of the horizon conjecture which explains the emergence of conformal symmetry near supersymmetric Killing horizons. The classification of warped AdS backgrounds that preserve more than 16 supersymmetries in $d=11$ and $d=10$ type II supergravities is also included. As part of the review, many of the proofs of key statements and examples given in the literature have been extensively reworked. As a result, their description has become more concise and shorter than that in their original exposition.

The two methods that have been extensively used to classify supersymmetric backgrounds, and which will be reviewed here, are the bilinears or G-structure method proposed by Gauntlett, Gutowski, Hull, Pakis and Reall [8] and the spinorial geometry method proposed by Gillard, Gran and Papadopoulos [9]. The use of these two methods is sufficient to describe all results that have been obtained in the literature, apart from the maximally supersymmetric solutions which are classified using a technique introduced in [10]. Both methods are explained in a simple example, the solution of the gaugino KSE on $\mathbb{R}^{6}$, where their individual features are illustrated. Moreover, the solution of the KSE of minimal $\mathcal{N}=1 d=5$ supergravity is described employing both methods. The spinorial geometry method in particular is used in the classification of backgrounds which preserve a near maximal number of supersymmetries.

Apart from the description of the two methods, the classification of supersymmetric solutions of minimal $\mathcal{N}=2 d=4$, $\mathcal{N}=1, d=4, \mathcal{N}=1 d=5$ and $\mathcal{N}=(1,0) d=6$ supergravities will be presented. In addition, the solution of the KSEs of heterotic supergravity will be described together with general theorems on the existence of certain classes of solutions. Aspects of the supersymmetric solutions of $d=11$ and $d=10$ type II supergravities will be explained. These include the solutions of the KSEs for one and nearly maximal number of Killing spinors. The classification of nearly maximal and maximal 
supersymmetric backgrounds of $d=11$ and $d=10$ type II supergravities will also be given. As an application we present the horizon conjecture and its proof in the context of $d=11$ supergravity which amongst other things demonstrates that $S L(2, \mathbb{R})$ generically emerges as a symmetry of near horizon geometries. The review will conclude with the computation of the Killing superalgebras of warped AdS backgrounds, and the proof of existence and uniqueness theorems for AdS backgrounds that preserve more than 16 supersymmetries.

\section{Methods for solving KSEs}

\subsection{KSEs and supersymmetry}

The KSEs of supergravity theories are the vanishing conditions of the supersymmetry variations of the fields. These are evaluated in the sector where all fermions vanish, which in turn implies that the supersymmetry variations of the bosons are identically zero. The remaining equations are a parallel transport equation for the supercovariant connection, $\mathcal{D}$, which is associated with the supersymmetry variation of the gravitino, $\psi$, and some algebraic equations which are associated with the supersymmetry variations of the remaining fermions, $\lambda$. In particular, one has

$$
\left.\delta \psi_{M}\right|_{\psi, \lambda=0}=\mathcal{D}_{M} \epsilon=0,\left.\quad \delta \lambda\right|_{\psi, \lambda=0}=\mathcal{A} \epsilon=0,
$$

where the spinor indices have been suppressed,

$$
\mathcal{D}_{M}:=\nabla_{M}+\sigma_{M}(e, F),
$$

is the supercovariant connection, $\nabla$ is the spin connection of the spacetime acting on the spinors,

$$
\nabla_{M}:=\partial_{M}+\frac{1}{4} \Omega_{M, A B} \Gamma^{A B},
$$

and $\sigma(e, F)$ is a Clifford algebra element which depends on the spacetime coframe $e$ and the fluxes $F$. The expression of $\sigma(e, F)$ in terms of the fields is theory dependent. The second KSE in (1) does not involve derivatives on $\epsilon$, i.e. it is algebraic, and $\mathcal{A}$ is a Clifford algebra element that depends on the fields. We use the notation, unless otherwise is explicitly stated, that capital Latin letters from the middle of the alphabet and onwards denote spacetime indices while capital Latin letters from the beginning of the alphabet denote coframe indices, i.e. the relation between the spacetime metric $g$ and the coframe $e$ is $g_{M N}=\eta_{A B} e_{M}^{A} e_{N}^{B}$. The spinor $\epsilon$ should be thought of as the parameter of the supersymmetry transformations and is taken to be commuting, see Appendix B for our spinor conventions.

The KSEs (1) are clearly linear in $\epsilon$ and at most first order.

- The solutions of the field equations of supergravity theories that admit a non-vanishing $\epsilon$ which satisfies (1) are called supersymmetric.

- The number, $N$, of supersymmetries preserved by a background is the number of linearly independent solutions $\epsilon$ that the KSEs (1) admit when they are evaluated on the fields of the background.

Generically, there are always solutions which do not preserve any supersymmetry. Conversely, the maximal number of supersymmetries that a background can preserve is the number of supersymmetry charges of the theory.

\subsection{Holonomy and gauge symmetry}

To understand some of the properties of the KSEs, it is instructive to investigate their integrability conditions. The first order integrability conditions can be written schematically as

$$
\mathcal{R}_{\mathrm{MN}} \epsilon:=\left[\mathcal{D}_{\mathrm{M}}, \mathcal{D}_{N}\right] \epsilon=0,\left[\mathcal{D}_{M}, \mathcal{A}\right] \epsilon=0,[\mathcal{A}, \mathcal{A}] \epsilon=0,
$$

where $\mathcal{R}$ is the curvature of the supercovariant connection. As we shall describe later these integrability conditions are also used in the investigation of the field equations of supersymmetric backgrounds.

It is natural to focus first on the gravitino KSE, which is a parallel transport equation. For a d-dimensional spacetime, the (reduced) holonomy group, $\operatorname{hol}(\nabla)$, of the spin connection $\nabla$ is contained in $\operatorname{Spin}(d-1,1)$. However because of the presence of fluxes, and in particular of the sigma term in (201), the holonomy of the supercovariant connection, $\operatorname{hol}(\mathcal{D})$, is contained in an SL group rather than a Spin group. In particular, the (reduced) holonomy of the supercovariant connections of generic $d=11$ [11-13] and type II supergravity backgrounds [14] is contained in $\operatorname{SL}(32, \mathbb{R})$. A list of the holonomies of lower dimensional supergravities can be found in [15].

To see this, note that the Lie algebra of $\operatorname{hol}(\mathcal{D})$ is computed by evaluating the supercovariant curvature $\mathcal{R}$ and its covariant derivatives $\mathcal{D}^{k} \mathcal{R}$ on spacetime vector fields, and then look at the span of the resulting expressions. In particular for $d=11$ supergravity $\mathcal{R}$ is given in (E.3). Observe that $\mathcal{R}(X, Y)$, for any two vector field $X$ and $Y$, is a general Clifford algebra element as it is expanded in all possible skews-symmetric products of gamma matrices apart perhaps from that of the zeroth order. As a consequence of Clifford algebra representation theory, the Lie bracket algebra of all skew-symmetric products of gamma matrices of degree 1 and above is $\mathfrak{s l}(32, \mathbb{R})$ in $d=11$. This in turn leads to the assertion that $\operatorname{hol}(\mathcal{D})$ is $S L(32, \mathbb{R})$ as mentioned above. 
This property of the holonomy of the supercovariant connection has important implications in understanding the geometry of supersymmetric backgrounds, see e.g. Section 8.5. An immediate consequence is that standard techniques, like the Berger classification, which are used to investigate the geometry of manifolds that admit parallel spinors with respect to the Levi-Civita connection do not apply. As a result a new approach is needed to investigate the solutions of KSEs and determine the geometry of solutions that admit Killing spinors.

Another property of the KSEs, which is essential in understanding the supersymmetric solutions, is the gauge symmetry. The gauge transformations of the Killing spinor equations are defined as the local transformations which transform a spacetime coframe $e$, fluxes $F$ and spinor $\epsilon$ but leave the KSEs (1) covariant, i.e.

$$
\ell \mathcal{D}(e, F) \ell^{-1}=\mathcal{D}\left(e^{\ell}, F^{\ell}\right), \quad \ell \mathcal{A}(\mathbf{e}, F) \ell^{-1}=\mathcal{A}\left(e^{\ell}, F^{\ell}\right) .
$$

The gauge group $G$ of most supergravity theories is smaller than the $\operatorname{hol}(\mathcal{D})$ of generic backgrounds, and always contains $\operatorname{Spin}(d-1,1)$ as a subgroup. This will be one of the ingredients of the spinorial geometry method.

\subsection{The spinor bilinears or G-structure method}

The bilinears or $G$-structure method was the first one to be used to systematically find all the solutions of minimal $\mathcal{N}=1$ $d=5$ supergravity in [8]. It is based on the observation that for spinors $\epsilon_{1}$ and $\epsilon_{2}$, one can associate a k-form,

$$
\tau=\frac{1}{k !} \bar{\epsilon}_{1} \Gamma_{M_{1} M_{2} \ldots M_{k}} \epsilon_{2} d x^{M_{1}} \wedge d x^{M_{2}} \wedge \cdots \wedge d x^{M_{k}},
$$

which is clearly bilinear in the spinors $\epsilon_{1}$ and $\epsilon_{2}$, where any $\operatorname{Spin}(d-1,1)$ invariant inner product can be used instead of the Dirac inner product indicated here, see Appendix B. From here on, we shall refer to these forms either as "k-form bilinears" or simply "bilinears". The 1 -form bilinear is the familiar Dirac current.

The existence of parallel spinors on simply connected Riemannian manifolds is equivalent to the existence of parallel forms. Indeed, $\nabla \epsilon=0$ implies that $\nabla \tau=0$, where $\tau$ is any form constructed as a bi-linear of the parallel spinors. Conversely, the existence of certain parallel forms imply that the holonomy of the Levi-Civita connection, hol $(\nabla)$, reduces to a subgroup of $S O(d)$. Then the spinor representations decomposed under $h o l(\nabla)$ have singlets which correspond to the parallel spinors.

This way of solving parallel transport equations for spinors can be adapted to the context of supergravity. One of the ingredients is to turn the KSEs into equations for the form bilinears $\tau$. In particular, the gravitino and the algebraic KSEs in (1) imply that

$$
\begin{aligned}
\nabla_{A} \tau_{B_{1} \ldots B_{k}}-\bar{\epsilon}_{1} \Gamma_{B_{1} \ldots B_{k}} \sigma_{A} \epsilon_{2}-\bar{\epsilon}_{1} \sigma_{A} \Gamma_{B_{1} \ldots B_{k}} \epsilon_{2} & =0, \\
\bar{\epsilon}_{1} \Gamma_{B_{1} \ldots B_{k}} \mathcal{A} \epsilon_{2} & =0,
\end{aligned}
$$

respectively, for every pair of Killing spinors $\epsilon_{1}$ and $\epsilon_{2}$. Expanding the $\sigma$ and $\mathcal{A}$ dependent parts in skew-symmetric powers of gamma matrices, the above equations can be expressed as equations for the form bi-linears $\tau$ of $\epsilon$, their covariant derivatives $\nabla \tau$, and the fluxes of the supergravity theory. The resulting equations that typically contain bilinears of different degree are solved to express some of the fluxes in terms of the form bilinears $\tau$ and their spacetime derivatives. In addition, one also finds conditions on the spinor bi-linears themselves. These are interpreted as the geometric conditions on the spacetime geometry required so that it admits a Killing spinor $\epsilon$.

Another ingredient that it is used to understand the geometry and topology of spacetime and to solve (7) are the algebraic relations between the spinor bi-linears $\tau$. These arise as a result of Fierz identities. In particular, these can be used to relate the wedge products of the various form bilinears. In turn, these provide information about the topological $G$-structure of the underlying manifold. Because of this, this method of solving KSEs is also referred to as the $G$-structures method. An illustration of how the method works will be given in Section 2.5 to solve the KSE of $d=6$ gauge theory.

\subsection{The spinorial geometry method}

Spinorial geometry [9] is a method for solving the KSEs working directly with the spinors. It is based on three ingredients. The first is the gauge symmetry of the KSEs, the second is a description of spinors in terms of multi-forms and third an oscillator basis in the space of Dirac spinors. These three ingredients can be used to solve the KSEs as follows.

As the Killing spinor equations admit a gauge invariance, it is natural to identify two backgrounds which are related by such transformations. As a result, the gauge symmetry can be used to set the Killing spinors in a normal or canonical form. This is equivalent to choosing representatives of the orbits of the gauge group of the theory on the space of spinors.

The description of spinors in terms of multi-forms is used to explicitly give the canonical forms of the Killing spinors up to a gauge transformation and leads to a simplification of the computations. This realization of spinors in terms of multi-forms is described in Appendix B for both Euclidean and Lorentzian signatures.

Furthermore, an oscillator basis in the space of Dirac spinors, together with the linearity of the Killing spinor equations, are utilized to express the Killing spinor equation as a linear system in terms of the fluxes and the geometry. The latter is represented by components of the spin connection of spacetime. This system is solved to express some of the fluxes in terms of the geometry and to find the conditions on the geometry required for the existence of Killing spinors. 
The solution of the linear system for both the fluxes and geometry can always be organized in representations of the isotropy group of the Killing spinors in the gauge group of the supergravity theory under study. This is the case even when the linear system is not manifestly expressed in representations of the isotropy group of the Killing spinors but instead in representations of a subgroup.

In the spinorial geometry approach, there is also a spacetime coframe, the "spinorial geometry coframe", adapted to the choice of the Killing spinor representatives and to the spinor oscillator basis that is used to solve the KSEs. The solution of the linear system is initially expressed in this coframe. Typically, the conditions on the spacetime geometry can be re-expressed as differential relations between the form bilinears of the Killing spinors. Similarly, the solution for the fluxes can be given in terms of the form bilinears, their derivatives and the metric.

As spinorial geometry is rather efficient for solving KSEs for a small as well as a large number $N$ of supersymmetries, many of the results in this review have been described in this method. However, the illustrative example below, as well as the solution of the KSEs of minimal $d=5$ supergravity in Section 5 , have been described employing both methods to provide a description of both approaches.

\subsection{A gauge theory example}

Before we proceed to describe the solution of the KSEs of supergravity theories, it is instructive to provide a simple example to illustrate how the bilinears and spinorial geometry methods work. For this consider the gaugino KSE

$$
F_{A B} \Gamma^{A B} \epsilon=0,
$$

on $\mathbb{R}^{6}$ equipped with the standard Euclidean metric, where we have suppressed the gauge and spinor indices and $\epsilon$ is a constant Weyl spinor, $\epsilon \in{ }^{c} \Delta^{+}$.

\subsubsection{Solution using bilinears}

To solve the gaugino KSE (8) in the bilinears method, consider the Fierz identity given by

$$
\left\langle\epsilon_{1}, \epsilon_{2}\right\rangle\left\langle\epsilon_{3}, \epsilon_{4}\right\rangle=\frac{1}{4}\left\langle\epsilon_{1}, \epsilon_{4}\right\rangle\left\langle\epsilon_{3}, \epsilon_{2}\right\rangle-\frac{1}{8}\left\langle\epsilon_{1}, \Gamma_{A B} \epsilon_{4}\right\rangle\left\langle\epsilon_{3}, \Gamma^{A B} \epsilon_{2}\right\rangle,
$$

where $\epsilon_{1}, \epsilon_{2}, \epsilon_{3}, \epsilon_{4} \in{ }^{c} \Delta^{+}\left(\mathbb{R}^{6}\right)$ and thus satisfy $\Gamma_{7} \epsilon_{r}=i \epsilon_{r}, r=1,2,3,4$ with respect to chirality operator, $\Gamma_{7}:=\Gamma_{1} \cdots \Gamma_{6}$, and indices are raised and lowered with the Euclidean metric. This is also equivalent to

$$
\left\langle\epsilon_{3}, \epsilon_{4}\right\rangle \epsilon_{2}=\frac{1}{4}\left\langle\epsilon_{3}, \epsilon_{2}\right\rangle \epsilon_{4}-\frac{1}{8}\left\langle\epsilon_{3}, \Gamma^{A B} \epsilon_{2}\right\rangle \Gamma_{A B} \epsilon_{4}
$$

for $\epsilon_{2}, \epsilon_{3}, \epsilon_{4} \in{ }^{c} \Delta^{+}\left(\mathbb{R}^{6}\right)$.

To proceed with the analysis, suppose that $\epsilon \in{ }^{c} \Delta^{+}\left(\mathbb{R}^{6}\right)$. We define the real 2-form $\omega$

$$
\omega=\frac{i}{2}\left\langle\epsilon, \Gamma_{M N} \epsilon\right\rangle d x^{M} \wedge d x^{N} .
$$

For convenience, we choose the normalization $\|\epsilon\|=1$. Then, on setting $\epsilon_{1}=\epsilon_{2}=\epsilon_{3}=\epsilon_{4}=\epsilon$ in (9), we find

$$
\omega_{A B} \omega^{A B}=6,
$$

and on setting $\epsilon_{2}=\epsilon_{3}=\epsilon_{4}=\epsilon$ in (10), one obtains

$$
\omega_{M N} \Gamma^{M N} \epsilon=6 i \epsilon .
$$

Next, substituting $\epsilon_{1}=\epsilon_{3}=\epsilon$ and $\epsilon_{2}=\Gamma_{A}^{L} \epsilon, \epsilon_{4}=\Gamma_{B L} \epsilon$ in (9), and making use of (12) together with the convention

$$
\Gamma_{L_{1} \ldots L_{6}} \epsilon=i \epsilon_{L_{1} \ldots L_{6}} \epsilon
$$

one derives the condition

$$
\omega_{A}{ }^{L} \omega_{B L}=\delta_{A B} .
$$

Therefore, on defining $I$ by $\omega_{A B}=\delta_{A C} I_{B}{ }_{B}, I$ is a complex structure on $\mathbb{R}^{6}$.

In order to find the conditions on $F$ for the gaugino $\operatorname{KSE}(8)$ to admit a Killing spinor, first note that this condition implies that

$$
F_{A B}\left\langle\epsilon, \Gamma^{A B} \epsilon\right\rangle=0,
$$

and hence

$$
F_{A B} \omega^{A B}=0 .
$$

Furthermore, (8) also implies that

$$
F_{C_{1} C_{2}}\left\langle\epsilon, \Gamma_{A B} \Gamma^{C_{1} C_{2}} \epsilon\right\rangle=0 \text {. }
$$


On taking the imaginary part of this identity, we find

$$
F_{C D} I^{C} I^{D}{ }_{B}=F_{A B} \text {. }
$$

The conditions (17) and (19) imply that $F$ is traceless and $(1,1)$ with respect to $I$, i.e. $F$ satisfies the Hermitian-Einstein condition on $\mathbb{R}^{6}$.

The conditions (17) and (19) are also sufficient to ensure that there is a solution $\epsilon \neq 0$ to (8) with no further conditions imposed on $F$. This can be straightforwardly shown by computing

$$
\left\|F_{A B} \Gamma^{A B} \epsilon\right\|^{2}=2 F^{2}-\left\langle\epsilon, F_{A B} F_{C D} \Gamma^{A B C D} \epsilon\right\rangle .
$$

The Fierz identities (12) and (13) together with (14) imply that

$$
*(\omega \wedge \omega)=2 \omega,
$$

from which it follows that

$$
\left\langle\epsilon, \Gamma_{A B C D} \epsilon\right\rangle=-\frac{1}{2}(\omega \wedge \omega)_{A B C D} .
$$

On substituting (22) into (20) and making use of the conditions (17) and (19), one can show that $\left\|F_{A B} \Gamma^{A B} \epsilon\right\|=0$, and hence (8) holds.

\subsubsection{Solution using spinorial geometry}

As $\operatorname{Spin}(6)=S U(4)$, the Weyl representation can be identified with the fundamental representation of $S U(4)$ on $\mathbb{C}^{4}$. Clearly (8) is covariant under rigid Spin(6) transformations. This can be used to choose $\epsilon$ as follows. Observe that Spin(6) has a single type of non-trivial orbit on $\mathbb{C}^{4}$ which is $S^{7}$. As a result $\epsilon$ can be chosen to lie along any direction in $\mathbb{C}^{4}$. Identifying $\mathbb{C}^{4}={ }^{c} \Delta^{+}\left(\mathbb{R}^{6}\right)=\Lambda^{* \mathrm{ev}}\left(\mathbb{C}^{3}\right)$, see Appendix B, one can choose $\epsilon$ as $\epsilon=1$.

In such a case, the gaugino $\operatorname{KSE}(8)$ can be written in the oscillator basis of Appendix B as

$$
\left(F_{\bar{\alpha} \bar{\beta}} \gamma^{\bar{\alpha} \bar{\beta}}+2 F_{\alpha \bar{\beta}} \delta^{\alpha \bar{\beta}}\right) 1=0,
$$

where we have used that $\gamma^{\alpha} 1=\sqrt{2} i_{e_{\alpha}} 1=0$. This implies that

$$
F_{\bar{\alpha} \bar{\beta}}=0, \quad F_{\alpha \bar{\beta}} \delta^{\alpha \bar{\beta}}=0,
$$

which can be recognized as the Hermitian-Einstein conditions on $F$ written in complex coordinates on $\mathbb{R}^{6}$. If $F$ is real, then $F_{\alpha \beta}=0$ and so in the language of complex geometry $F$ is a $(1,1)$ - and Hermitian traceless form.

Clearly the conditions (24) on $F$ are written in representations of the $S U(3)$ isotropy group of the Killing spinor 1 . They can also be written covariantly after using the 2 -form bilinear

$$
\omega=\frac{i}{2}\left\langle 1, \Gamma_{M N} 1\right\rangle d x^{M} \wedge d x^{N}=-i \delta_{\alpha \bar{\beta}} d z^{\alpha} \wedge d z^{\bar{\beta}},
$$

where $z$ are complex coordinates on $\mathbb{R}^{6}$ with respect to the complex structure $I$ defined by $\omega_{A B}=\delta_{A C} I_{B}{ }_{B}$. In particular, the conditions (24) can be rewritten as (17) and (19). This type of procedure for finding solutions to the linear system presented above can also be applied to all linear systems that arise in the solutions of the KSEs of supergravity theories.

\section{Minimal $\mathcal{N}=2 d=4$ supergravity}

The bosonic fields of the gravitational multiplet are a metric and an abelian 2-form gauge field strength $F, d F=0$. The bosonic action is the Einstein-Maxwell system. Since it describes the long range force fields of the cosmos, it has been extensively investigated and its solutions include black holes with electric and/or magnetic charges and gravitational waves. It also arises in various limits of higher dimensional theories which include string- and M-theory. As a result, many of the brane configurations of these theories reduce upon dimensional reduction to solutions of this minimal $\mathcal{N}=2$ supergravity.

Furthermore, this is the theory for which the KSE was first solved in full generality [7] using twistorial techniques. Because of this, we shall begin the investigation of gravitational theories with solving the KSE of this theory. Here, we shall present the analysis employing the spinorial geometry method.

\subsection{Fields and Killing spinors}

\subsubsection{KSE and field equations}

The only fermionic field in the theory is a gravitino whose supersymmetry variation gives the KSE

$$
\mathcal{D}_{M} \epsilon=0,
$$

where the supercovariant connection, $\mathcal{D}$, is

$$
\mathcal{D}_{M}:=\nabla_{M}+\frac{i}{4} F_{A B} \Gamma^{A B} \Gamma_{M},
$$

$\nabla$ is the spin connection of the spacetime and the supersymmetry parameter $\epsilon$ is a Dirac spinor of $\operatorname{Spin}(3,1)$. 
The supercovariant curvature, $\mathcal{R}$, is

$$
\begin{aligned}
\mathcal{R}_{M N}:=\left[\mathcal{D}_{M}, \mathcal{D}_{N}\right]= & \frac{1}{4} R_{M N, A B} \Gamma^{A B}-\frac{1}{2} F_{M A} F_{N B} \Gamma^{A B}-\frac{1}{2}{ }^{*} F_{M A}{ }^{*} F_{N B} \Gamma^{A B} \\
& \left.-\frac{1}{2} i \Gamma_{A B}\left[{ }_{M} \nabla_{N}\right]^{A B}-i \nabla_{[M} F_{N}\right]^{A} \Gamma_{A},
\end{aligned}
$$

where $R$ is the Riemann tensor of the spacetime which arises from the spin connection term $\nabla$ in $\mathcal{D}$ and ${ }^{*} F$ is the Hodge dual of $F,{ }^{*} F_{A B}=(1 / 2) F_{C D} \epsilon^{C D}{ }_{A B}$, with $\epsilon^{0123}=1$. To derive (28), one also uses $d F=0$.

For generic backgrounds the (reduced) holonomy of $\mathcal{D}$ is contained in $\operatorname{SL}(2, \mathbb{H})$ [15]. The enhancement in holonomy from $\operatorname{Spin}(3,1)$ of $\nabla$ to $S L(2, \mathbb{H})$ of $\mathcal{D}$ is due to the linear and cubic terms in gamma matrices in the expression for $\mathcal{R}$ above. This is a characteristic property of many supergravity theories and some of its consequences will be explained below in Section 3.3.2, see also Section 8.5 .

The field equations of the theory can be derived from the supercovariant curvature $\mathcal{R}$. In particular one has

$$
\Gamma^{N} \mathcal{R}_{M N}=-\frac{1}{2} E_{M N} \Gamma^{N}-\frac{i}{2} L F_{N} \Gamma^{N} \Gamma_{M},
$$

where

$$
E_{M N}:=R_{M N}-2 F_{P M} F_{N}^{P}+\frac{1}{2} F^{2} g_{M N}=0, \quad L F_{M}:=\nabla^{N} F_{N M}=0,
$$

are the Einstein and Maxwell field equations, respectively. It is significant for the investigation of solutions that some of the components of $\mathcal{R}$ are proportional to the field equations. This will be used to demonstrate that some of the field equations are implied from the KSEs.

\subsubsection{Killing spinors}

To solve the KSEs in the spinorial geometry approach, [9], one has to choose a normal form for the Killing spinors. As described in Appendix B, the space of Dirac spinors can be identified with $\Lambda^{*}\left(\mathbb{C}^{2}\right)$. The Weyl representation of $\operatorname{Spin}(3,1)=$ $\operatorname{SL}(2, \mathbb{C})$ is the fundamental representation of $\operatorname{SL}(2, \mathbb{C})$ on $\mathbb{C}^{2}$. The chiral and anti-chiral spinors are identified with the even degree, $\Lambda^{\mathrm{ev}}\left(\mathbb{C}^{2}\right)$, and odd degree, $\Lambda^{\text {odd }}\left(\mathbb{C}^{2}\right)$, forms, respectively. Observe though that in contrast to the spinors of $\mathcal{N}=1$ $d=4$ supergravity that will be investigated next, the chiral and anti-chiral representations are not complex conjugate to each other as the components of a Dirac spinor are independent. Let us assume without loss of generality that the positive chirality component of $\epsilon$ does not vanish. As $S L(2, \mathbb{C})$ acts transitively on $\mathbb{C}^{2}-\{0\}$, the positive chirality component of $\epsilon$ can always be chosen as the spinor 1 . The isotropy group of the spinor 1 in $S L(2, \mathbb{C})$ is $\mathbb{C}$ whose Lie algebra is spanned by $\left\{\gamma^{1-}, \gamma^{1-}\right\}$, see Appendix B. The most general anti-chiral component of $\epsilon$ is $a e_{1}+b e_{2}$ for $a, b \in \mathbb{C}$. If $b \neq 0$, then the $\mathbb{C}$ isotropy group can be used to set $a=0$. Thus the first Killing spinor can locally be chosen as

$$
\text { either } \epsilon=1+b e_{2} \text {, or } \epsilon=1+a e_{1} \text {, }
$$

where $a, b$ become complex-valued functions on spacetime. The isotropy groups of $1+b e_{2}$ and $1+a e_{1}$ are $\{1\}$ and $\mathbb{C}$ in $S L(2, \mathbb{C})$, respectively. As we shall demonstrate, the two Killing spinors give rise to two distinct types of geometries on spacetime; one is associated with a time-like Killing vector field and the other with a null one. Because of this, they are also referred to as the time-like and null cases, respectively.

Before we proceed with the solution of the gravitino $\operatorname{KSE}(26)$, observe that it is linear over $\mathbb{C}$. This means that if $\epsilon$ is a Killing spinor, then $i \epsilon$ will also be a Killing spinor. Furthermore, if $\epsilon$ is a Killing spinor, then both $r_{\mathrm{B}} \epsilon=-\Gamma_{3} * \epsilon$ and $i r_{\mathrm{B}} \epsilon=-i \Gamma_{3} * \epsilon$ will also be Killing spinors because $r_{\mathrm{B}}$ commutes with the KSE, see Appendix B for the definition of $r_{\mathrm{B}}$. As these four spinors are linearly independent over $\mathbb{R},(26)$ admits either four or eight Killing spinors as solutions. Therefore the Einstein-Maxwell system admits supersymmetric solutions which preserve either half or all of the supersymmetry of the theory.

3.2. Case 1: $\epsilon=1+b e_{2}$

\subsubsection{Solution of linear system}

To construct the linear system apply the gravitino $\operatorname{KSE}(26)$ to the spinor $\epsilon=1+b e_{2}$ and expand the resulting expression in the basis $\left\{1, e_{12}, e_{1}, e_{2}\right\}$ in the space of Dirac spinors. The vanishing of each component in this basis yields

$$
\begin{aligned}
-\frac{1}{2} \Omega_{M,+-}+\frac{1}{2} \Omega_{M, 1 \overline{1}}+\frac{i}{\sqrt{2}} b\left(F_{-M}-i^{*} F_{-M}\right) & =0, \\
-\Omega_{M,+\overline{1}}+\frac{i}{\sqrt{2}} b\left(F_{\overline{1} M}-i^{*} F_{\overline{1} M}\right) & =0, \\
-b \Omega_{M,-\overline{1}}+\frac{i}{\sqrt{2}}\left(F_{\overline{1} M}+i^{*} F_{\overline{1} M}\right) & =0, \\
\partial_{M} b+\frac{b}{2}\left(\Omega_{M,+-}+\Omega_{M, 1 \overline{1}}\right)+\frac{i}{\sqrt{2}}\left(F_{+M}+i^{*} F_{+M}\right) & =0,
\end{aligned}
$$

where $* F$ is the Hodge dual of $F$ and $\epsilon_{-+1 \overline{1}}=-i$. 
This linear system can be solved to give

$$
\begin{aligned}
\Omega_{+,-+} & =\partial_{+} \log b+\partial_{+} \log \bar{b}, \quad \Omega_{-,-+}=0, \quad \Omega_{1,-+}=\partial_{1} \log \bar{b}, \\
\Omega_{+,-\overline{1}} & =\Omega_{\overline{1},-\overline{1}}=0, \quad \Omega_{-,-\overline{1}}=-(b \bar{b})^{-1} \partial_{\overline{1}} \log b, \quad \Omega_{1,-\overline{1}}=\partial_{-} \log b, \\
\Omega_{+,+\overline{1}} & =-b \partial_{\overline{1}} \bar{b}, \quad \Omega_{-,+\overline{1}}=\Omega_{\overline{1},+\overline{1}}=0, \quad \Omega_{1,+\overline{1}}=\partial_{+} \log \bar{b}, \\
\Omega_{+, 1 \overline{1}} & =\partial_{+} \log \bar{b}-\partial_{+} \log b, \quad \Omega_{-, 1 \overline{1}}=0, \quad \Omega_{1,1 \overline{1}}=\partial_{1} \log \bar{b},
\end{aligned}
$$

with

$$
\partial_{+} b=|b|^{2} \partial_{-} b,
$$

and

$$
\begin{aligned}
F=- & \frac{i}{\sqrt{2}} \partial_{-}(b-\bar{b}) \mathbf{e}^{-} \wedge \mathbf{e}^{+}+\frac{i}{\sqrt{2}} \partial_{-}(b+\bar{b}) \mathbf{e}^{1} \wedge \mathbf{e}^{\overline{1}} \\
& +i \frac{1}{\sqrt{2}}|b|^{-2}\left(\partial_{\overline{1}} b \mathbf{e}^{\overline{1}}-\partial_{1} \bar{b} \mathbf{e}^{1}\right) \wedge \mathbf{e}^{-}+i \frac{1}{\sqrt{2}}\left(\partial_{\overline{1}} \bar{b} \mathbf{e}^{\overline{1}}-\partial_{1} b \mathbf{e}^{1}\right) \wedge \mathbf{e}^{+} .
\end{aligned}
$$

Therefore all of the components of the spin connection and those of the flux $F$ are determined in terms of the complex function $b$.

\subsubsection{Geometry}

To identify the geometry of the spacetime as a consequence of the conditions (33) that arise from the KSE, it is useful to consider the 1 -form bilinears of the Killing spinors $\epsilon$ and $\tilde{\epsilon}=\Gamma_{3} * \epsilon$. These can easily be computed to find that the linearly independent ones can be chosen as

$$
\begin{aligned}
& X=\frac{1}{2} D\left(\epsilon, \Gamma_{A} \epsilon\right) \mathbf{e}^{A}=\frac{1}{\sqrt{2}}\left(|b|^{2} \mathbf{e}^{+}-\mathbf{e}^{-}\right), \\
& W^{2}=\frac{1}{2} D\left(\epsilon, \Gamma_{5} \Gamma_{A} \epsilon\right) \mathbf{e}^{A}=\frac{1}{\sqrt{2}}\left(|b|^{2} \mathbf{e}^{+}+\mathbf{e}^{-}\right), \\
& W^{3}+i W^{1}=-\frac{1}{2} D\left(\tilde{\epsilon}, \Gamma_{5} \Gamma_{A} \epsilon\right) \mathbf{e}^{A}=\sqrt{2} i b \mathbf{e}^{1},
\end{aligned}
$$

which give rise to four real 1-forms $X, W^{1}, W^{2}, W^{3}$ on spacetime. These are orthogonal and $X$ is timelike, $g(X, X)=-|b|^{2}$, and the remaining three are spacelike, $g\left(W^{i}, W^{j}\right)=|b|^{2} \delta^{i j}$.

The conditions on the geometry can be rewritten in terms of the 1 -form bilinears as

$$
\mathcal{L}_{X} g=0, d W^{i}=0,
$$

i.e. $X$ is Killing and $W^{i}$ are closed. Furthermore, a consequence of $d F=0$ and (35) is that $\mathcal{L}_{X} F=0$ and therefore the flux $F$ is invariant as well. It can be shown that $\mathcal{L}_{X} \epsilon=0$, where $\mathcal{L}_{X}$ is the spinorial Lie derivative

$$
\mathcal{L}_{X}=\nabla_{X}+\frac{1}{8}(d X)_{A B} \Gamma^{A B},
$$

along the Killing vector field $X$. The significance of $\mathcal{L}_{X} \epsilon=0$ will become apparent in the description of the Killing superalgebras of supersymmetric backgrounds.

From here on, we denote by $X$ both the 1 -form bilinears of the Killing spinors and their associated vector fields which leave all fields of supersymmetric backgrounds invariant. Such an identification is justified because the spacetime metric induces an isomorphism between the cotangent and tangent bundles of a spacetime.

\subsubsection{Special coordinates}

One can introduce a set of local coordinates $\left(t, x^{i}\right)$ on the spacetime as $X=\partial_{t}$ and $W^{i}=d x^{i}$. (34) implies that $\partial_{t} b=0$. In these coordinates, the metric and flux $F$ are written as

$$
\begin{aligned}
& d s^{2}=-|b|^{2}\left(d t+\omega_{i} d x^{i}\right)^{2}+|b|^{-2} \delta_{i j} d x^{i} d x^{j}, \\
& F=-d(\operatorname{Im} b) \wedge\left(d t+\omega_{i} d x^{i}\right)+\frac{1}{2}|b|^{-2} *_{3} d(\operatorname{Re} b),
\end{aligned}
$$

where the Hodge duality operation $*_{3}$ is taken with respect to the Euclidean 3-metric and

$$
d \omega=-*_{3} Y, \quad Y_{i}=i|b|^{-2} \partial_{x^{i}} \log \frac{b}{\bar{b}} .
$$

Eqs. (39) and (40) summarize all the conditions on the fields implied by the KSE. 


\subsubsection{Solutions}

To find solutions, one has to solve the field equations and the Bianchi identity, $d F=0$, of the theory. However, the Einstein equation is implied by the KSE, the Maxwell equation of $F$ and $d F=0$. To see this, assuming the field equation for $F$ one has from the integrability condition of the $\operatorname{KSE}(29)$ that $E_{A B} \Gamma^{B} \epsilon=0$. Taking the Dirac inner product with the Killing spinor $\epsilon$, one deduces that

$$
E_{A B} X^{B}=0 .
$$

So everywhere that $|b| \neq 0, E_{A 0}=0$ as $X=|b| \mathbf{e}^{0}$ is along the coframe direction $\mathbf{e}^{0}$. Next acting on $E_{A B} \Gamma^{B} \epsilon=0$ with $E_{A C} \Gamma^{C}$ and using that $E_{A 0}=0$, one finds that

$$
E_{A i} E_{A}{ }^{i}=0 \text {, no summation over } A \text {, }
$$

as $\epsilon \neq 0$. In turn (41) and (42) imply that $E_{A B}=0$ and the Einstein equation is satisfied. Therefore to find solutions, one has to solve the field equation of $F$ and $d F=0$.

To find electric or magnetic solutions, one has to take $b$ to be imaginary or real, respectively. In such a case, either the field equation for $F$, or $d F=0$, implies that $|b|^{-1}$ is a harmonic function on $\mathbb{R}^{3}$. The solutions are static, $d \omega=0$. For $|b|^{-1}=1+Q /|x|$, one recovers the electric or magnetic extreme Reissner-Nordström black holes. If $|b|^{-1}$ is chosen to be a multi-centred harmonic function on $\mathbb{R}^{3}$, one finds the Majumdar and Papapetrou [16,17], multi-black hole solutions.

The theory admits dyonic black holes for $b=|b| e^{i \alpha}$, where $\alpha$ is a constant phase and $|b|^{-1}$ is a harmonic function on $\mathbb{R}^{3}$. The solutions are again static. It can be seen from (40) that these are the most general static black hole solutions that preserve some supersymmetry [18]. Rotating black hole solutions, $d \omega \neq 0$, have been considered by Israel, Wilson and Perjes [19,20]. These are the most general stationary supersymmetric black hole solutions [7] of the theory.

\subsection{Case $2: \epsilon=1+a e_{1}$}

\subsubsection{Solution of linear system}

Evaluating the KSE on $\epsilon=1+a e_{1}$, and expanding the resulting equation in the spinor basis $\left\{1, e_{1}, e_{2}, e_{12}\right\}$, one finds the linear system

$$
\begin{aligned}
& \frac{1}{2}\left(\Omega_{M,-+}+\Omega_{M, 1 \overline{1}}\right)+\frac{i}{\sqrt{2}} a\left(F_{1 M}-i^{*} F_{1 M}\right)=0, \\
& \Omega_{M, \overline{1}+}+\frac{i}{\sqrt{2}} a\left(-F_{+M}+i^{*} F_{+M}\right)=0, \\
& \partial_{M} a+\frac{1}{2} a\left(\Omega_{M,-+}-\Omega_{M, 1 \overline{1}}\right)+\frac{1}{\sqrt{2}}\left(F_{\overline{1}_{M}}+i^{*} F_{\overline{1} M}\right)=0, \\
& -a \Omega_{M, 1+}+\frac{i}{\sqrt{2}}\left(F_{+M}+i^{*} F_{+M}\right)=0,
\end{aligned}
$$

where ${ }^{*} F$ is the spacetime Hodge dual of $F$ as in (32).

Suppose first that $a \neq 0$. In such case, the non-vanishing components of the spin connection are $\Omega_{M,-1}, \Omega_{M,-\overline{1}}$ and

$$
\Omega_{-,-+}=-\partial_{-} \log (a \bar{a}+1), \quad \Omega_{-, 1 \overline{1}}=\frac{\bar{a} \partial_{-} a-a \partial_{-} \bar{a}}{a \bar{a}+1},
$$

with

$$
\partial_{1} a=\partial_{\overline{1}} a=\partial_{+} a=0,
$$

and the flux is given by

$$
F=-\frac{i}{\sqrt{2}(a \bar{a}+1)}\left(\partial_{-} \bar{a} \mathbf{e}^{1}-\partial_{-} a \mathbf{e}^{\overline{1}}\right) \wedge \mathbf{e}^{-} .
$$

On the other hand, if $a=0$, then $F=0$ and the non-vanishing components of the spin connection are $\Omega_{M,-1}$ and $\Omega_{M,-\overline{1}}$.

\subsubsection{Geometry}

To investigate the geometry of spacetime, one can compute the form bilinears or equivalently explore the restrictions on the coframe that arise from the vanishing conditions on the components of the spin connection. In particular one finds that the null 1-form bilinear

$$
X=(1+a \bar{a}) \mathbf{e}^{-},
$$

is $\nabla$-parallel, $\nabla_{A} X=0$. Therefore these backgrounds are pp-waves. The rest of the conditions on the spacetime can be recovered by asserting that

$$
\alpha=\beta \mathbf{e}^{-} \wedge \mathbf{e}^{1}, \quad \partial_{-} \log \beta=-2(1+a \bar{a})^{-1} a \partial_{-} \bar{a},
$$


is parallel, $\nabla_{A} \alpha=0$, with $\partial_{+} \beta=\partial_{1} \beta=\partial_{\overline{1}} \beta=0$. Therefore the full geometric content of spacetime is to admit a parallel real null 1 -form and a parallel complex null 2-form. The geometry of backgrounds with $a=0$ can be described in a similar way.

A feature of the geometry of supersymmetric backgrounds in $d=4$ is that the orbit type of the Killing spinor can change under parallel transport. This is due to the fact that the holonomy of the supercovariant connection is contained in $\operatorname{SL}(2, \mathbb{H})$ instead of $\operatorname{Spin}(3,1)$. So it is possible to begin with a Killing spinor with isotropy group $\{1\}$ and after parallel transport with the supercovariant connection $\mathcal{D}$ to end up with a Killing spinor with isotropy group $\mathbb{C}$. In such a case, the 1 -form bilinear $X$ will change from timelike to null. Such a phenomenon occurs in black hole solutions with a Killing horizon for which the stationary Killing vector field coincides with the vector bilinear. Therefore the description of the geometry here and in Section 3.2.2 is local. A more detailed discussion of this, and how it is related to G-structures, will be given in Section 8.5.

\subsubsection{Special coordinates}

As $d \mathbf{e}^{-}=0$, introduce a coordinate $v$ and set $\mathbf{e}^{-}=d v$. Furthermore, adapt a coordinate $u$ along $X, X=\partial_{u}$. As all the fields and form bilinears are invariant under $u$, a coframe can be chosen as

$$
\mathbf{e}^{-}=d v, \quad \mathbf{e}^{+}=(1+a \bar{a})\left(d u+V d v+n_{I} d y^{I}\right), \quad \mathbf{e}^{i}=e_{I}^{i} d y^{I}+p^{i} d v,
$$

where all components are $u$ independent. A further simplification is possible as the choice of the coframe $\left\{\mathbf{e}^{-}, \mathbf{e}^{+}, \mathbf{e}^{i}: i=\right.$ $1, \overline{1}\}$, is not unique. Indeed the local coframe rotation

$$
\mathbf{e}^{-} \rightarrow \mathbf{e}^{-}, \quad \mathbf{e}^{+} \rightarrow \mathbf{e}^{+}-q_{i} \mathbf{e}^{i}-\frac{1}{2} q^{2} \mathbf{e}^{-}, \mathbf{e}^{i} \rightarrow \mathbf{e}^{i}+q^{i} \mathbf{e}^{-},
$$

leaves all the geometric data invariant, including the form bilinears and the fields of the theory. The parameter $q$ is a local gauge transformation which takes values in the isotropy group $\mathbb{C}$ of the Killing spinor. A more formal treatment of this will be given in the discussion of the geometry of $d=6$ supergravity backgrounds in Section 6.2.1. Thus up to a possible rotation (50), one can choose a coframe (49) with $p=0$. The remaining description of the geometry and solutions will be conducted in such a coframe.

The condition $\nabla_{i} \alpha=0$ in (48) implies that the coframe $\left\{\mathbf{e}^{1}, \mathbf{e}^{\overline{1}}\right\}$ can be chosen to be independent of $y^{I}$. To summarize, the metric and flux can be chosen as

$$
\begin{aligned}
d s^{2} & =2(1+a \bar{a}) d v\left(d u+V d v+n_{I} d y^{I}\right)+\delta_{i j} e_{I}^{i}(v) e_{J}^{j}(v) d y^{I} d y^{J}, \\
F & =-\frac{i}{\sqrt{2}(a \bar{a}+1)}\left(\partial_{-} \bar{a} e_{I}^{1}(v)-\partial_{-} a e_{I}^{\overline{1}}(v)\right) d y^{I} \wedge d v,
\end{aligned}
$$

where $V$ can depend on both $v$ and $y$ coordinates and $\tilde{d} n$ depends only on $v$, where $\tilde{d} n:=\frac{1}{2}(d n)_{i j} \mathbf{e}^{i} \wedge \mathbf{e}^{j}$. The latter property arises after computing the spin connection in this coframe and comparing it with the second condition in (44).

\subsubsection{Solutions}

To find solutions, one has to solve the field equations and the Bianchi identity $d F=0$ of the theory. Observe that the field equation for $F$ and its Bianchi identity are automatically satisfied. Furthermore, some components of the Einstein equation are also implied from the KSE. The argument for this is similar to that presented in Section 3.2.4. In particular, one has from the integrability condition of the KSE (29) that $E_{A B} \Gamma^{B} \epsilon=0$. Taking the Dirac inner product with the Killing spinor $\epsilon$, one deduces that $E_{A B} X^{B}=0$. So $E_{A+}=0$ as $X=(1+a \bar{a}) \mathbf{e}^{-}$is along the coframe direction $\mathbf{e}^{-}$. Next, acting on $E_{A B} \Gamma^{B} \epsilon=0$ with $E_{A C} \Gamma^{C}$ and using that $E_{A+}=0$, one finds that $E_{A i} E_{A}{ }^{i}=0$ as $\epsilon \neq 0$, where there is no summation over the index $A$. Therefore all the field equations are satisfied provided that $E_{--}=0$.

A large class of solutions can be found after assuming in addition that $\partial_{v}$ leaves all the fields invariant, i.e. $a$ is constant. Then $F=0, \tilde{d} n=0$ and $\mathbf{e}^{i}=d y^{i}$. The spacetime can locally be viewed as a fibration having fibre $\mathbb{R}^{2}$ with coordinates $(u, v)$ over a base space $B^{2}=\mathbb{R}^{2}$ with coordinates $y^{i}$. The Einstein equation $E_{--}=0$ gives that

$$
\partial^{2} V=0,
$$

i.e. $V$ is a harmonic function on $\mathbb{R}^{2}$. A large class of solutions can be found for $V=Q \log |y|^{2}+A_{i j} y^{i} y^{j}$ and $n=\delta_{i j} y^{i} d y^{j}$, where $Q$ is a constant and the constant real matrix $\left(A_{i j}\right)$ is traceless, $\delta^{i j} A_{i j}=0$.

\subsection{Maximally supersymmetric solutions}

For maximally supersymmetric solutions the supercovariant curvature $\mathcal{R}$ in (28) must vanish. In particular the linear term in gamma matrices gives that $\nabla_{[M} F_{N]_{L}}=0$ which together with $d F=0$ imply that $\nabla F=0$. Thus $F$ is parallel. Then the terms quadratic in the gamma matrices in (28) imply that the spacetime curvature $R$ is parallel as well, $\nabla R=0$. Therefore the spacetime is a Lorentzian symmetric space and $F$ is an invariant 2-form. Lorentzian symmetric spaces, up to discrete identifications, are products of de-Sitter $d S_{n}$, anti-de-Sitter $A d S_{n}$, Cahen-Wallach $C W_{n}$ and Minkowski $\mathbb{R}^{n-1,1}$ spaces with Euclidean signature symmetric spaces [21]. A description of de-Sitter $d S_{n}$ and anti-de-Sitter $A d S_{n}$ spaces can be found in [22], and for the Cahen-Wallach spaces see Appendix C.2.

After some investigation, the maximal supersymmetric solutions of minimal $\mathcal{N}=2$ supergravity are locally isometric to 
- $A d S_{2} \times S^{2}$ with metric and flux

$$
\begin{aligned}
& d s^{2}=\ell^{2} d \dot{s}^{2}\left(A d S_{2}\right)+\ell^{2} d \dot{s}^{2}\left(S^{2}\right), \\
& F=\mu \operatorname{dvol}\left(A d S_{2}\right)+v \operatorname{diol}\left(S^{2}\right),
\end{aligned}
$$

with $\ell^{2}=\mu^{2}+v^{2}$ and $\ell, \mu, v \in \mathbb{R}, \ell \neq 0$.

- the plane wave $C W_{4}$ with metric and flux

$$
\begin{aligned}
& d s^{2}=2 d v d u+A_{i j} x^{i} x^{j} d v^{2}+\delta_{i j} d x^{i} d x^{j}, \\
& F=\mu_{i} d v \wedge d x^{i},
\end{aligned}
$$

with $A=-\mu^{2} \operatorname{diag}(1,1)$ and $\mu \neq 0$.

- and Minkowski spacetime $\mathbb{R}^{3,1}$ for which $F=0$.

where $d s^{2}$ and dvol denote the metrics and volume forms of the indicated spaces with radii normalized to one, respectively.

\subsection{Classification of non-minimal $\mathcal{N}=2$ supergravity solutions}

After the first classification of $\mathcal{N}=2$ supergravity solutions in [7], further extensions of this work to include dilaton and axion scalar fields were constructed in [23], using the same techniques. The solutions again split into timelike and null cases; and the timelike solutions have a metric whose form is identical to that given in (39), though the conditions on the terms appearing in the metric receive modifications if additional scalar fields are present. The next theory to be considered was the minimal gauged supergravity. It was known for some time that this theory contains dyonic black holes [24], so a systematic understanding of the supersymmetric solutions was of particular interest. In [25] the classification was performed using the bilinears method; the presence of the negative cosmological constant deforms the transverse 3-manifold, which appears in the timelike class of solutions as $\mathbb{R}^{3}$, to a more general class of 3-manifold, which admits a Riemannian Weyl structure. Other interesting solutions whose supersymmetry was investigated using these techniques are supersymmetric Plebanski-Demianski geometries, and the C-metric [26,27].

The minimal gauged supergravity analysis was then further extended in [28], both in terms of constructing new examples of solutions, and in terms of investigating solutions with extended supersymmetry. In particular, solutions of the ungauged theory preserve either $N=4$ or $N=8$ supersymmetry, whereas in the gauged theory $N=2, N=4, N=6$ and $N=8$ solutions are in principle allowed. It was shown in [28] by considering explicitly the integrability conditions of the KSE that all null solutions with $N=6$ solutions must be locally isometric to the unique maximally supersymmetric solution $A_{d} S_{4}$; this result was also shown to hold for the timelike class in [29], using spinorial geometry techniques. Further classification, via spinorial geometry, of the solutions with $N=4$ supersymmetry was done in [30], subject to the assumption, for solutions entirely in the timelike class, that the spinorial Lie derivative of the additional Killing spinor with respect to the isometry generated by the first spinor vanishes.

Numerous solutions have also been found for $\mathcal{N}=2$ supergravity coupled to vector multiplets. For the case of the ungauged theory, a large class of solutions in the timelike class, including electrically and magnetically charged black holes, were found in [31]. These solutions, for which the metric takes the same form as in the minimal theory (39), were found by proposing a particular ansatz. Later, it was shown in the classification of [32], using the bilinears method, that the timelike solutions found in [31] are in fact the most general possible, and all null solutions were also determined.

Black hole solutions in gauged supergravity coupled to vector multiplets were also constructed in [33,34] and [35]. The systematic classifications of solutions of gauged supergravity coupled to vector multiplets were constructed in [36] and [37]. In this case, solutions with $N=2$ supersymmetry were classified using spinorial geometry techniques, and in the timelike class the general form of the metric is again a $U(1)$ fibration over a 3-dimensional transverse manifold admitting a Riemannian Weyl structure. Using these results, novel examples of black holes were found [38,39]; further examples of supersymmetric asymptotically $\mathrm{AdS}_{4}$ black holes were considered in [40] and [41]. Solutions in the timelike class with extended $N=4$ supersymmetry were then classified using spinorial geometry techniques in [42], again subject to the assumption that the spinorial Lie derivative of the additional Killing spinor with respect to the isometry generated by the first spinor vanishes. A further generalization to include non-abelian vector multiplets, using the bilinears method, was made in [43].

Additional generalizations have also been made to include both vector and hypermultiplets. Supersymmetric solutions in the ungauged theory coupled to arbitrary vector and hypermultiplets were classified in [44]. One novel feature of the results of this work is that solutions in the timelike class no longer have a metric of the form given in (39), but instead have geometries which are $U(1)$ fibrations over a 3-manifold whose spin connection is determined by the pull-back of a certain quaternionic $S U(2)$ connection. The extension of this analysis to gauged supergravity coupled to vector and hypermultiplets was carried out in [45], and supersymmetric black holes coupled to hypermultiplets were constructed in [46], which also included a (partial) classification of solutions. These classifications all employ the bilinears method.

Higher derivative solutions have also been considered in specific examples. In [47], an ansatz for stationary solutions in supergravity coupled to vector and hypermultiplets, including higher derivative terms was considered. The conditions imposed by supersymmetry were derived, assuming that the Killing spinors satisfied a certain projection. It would be 
of interest to construct a systematic classification of supersymmetric solutions of higher derivative supergravity in four dimensions.

Supersymmetric solutions of various $\mathcal{N}>2$ theories have also been classified in [48], further generalizing the earlier analysis of [23]; as well as that in [49]. Theories with novel signature have been considered as well. The simplest case is minimal Euclidean supergravity with a single Maxwell field, [50] and [51], whose supersymmetric solutions were classified via spinorial geometry. Solutions of minimal gauged supergravity with $(2,2)$ signature were also classified using the bilinears method in [52]. These include geometries involving Gauduchon-Tod structures, which also appear in various other types of $d=4$ and $d=5$ supergravity theories with non-standard couplings, such as de-Sitter supergravities and other pseudosupergravities; we shall not consider such theories here.

\section{4. $\mathcal{N}=1 d=4$ supergravity}

Next we shall describe the solutions to the KSEs of $\mathcal{N}=1 d=4$ supergravity coupled to any number of (non-abelian) vector and scalar multiplets [53], see also [54] for the treatment of a special case. For the construction of the theory see [55] and references therein. This theory is one of the most phenomenologically attractive in the context of supersymmetry. Furthermore, as we shall demonstrate the KSEs can be solved exactly for any number of supersymmetries.

The bosonic fields of the theory, in addition to the metric, are the vector gauge potentials $A^{a}$ and the scalars $\phi^{i}$. The latter are functions on the spacetime with values in a Kähler manifold $S$. We shall refer to $S$ as the "scalar" or "sigma model" manifold. The Kähler geometry on $S$ arises as a consequence of the invariance of the action under the supersymmetry transformations of the theory. The rest of the relevant properties of the theory, including the couplings, will be described below along with the KSEs.

\subsection{Fields and spinors}

\subsubsection{Killing spinor equations}

The KSEs of $\mathcal{N}=1$ supergravity coupled to any number of (non-abelian) vector and matter multiplets can easily be read off from the supersymmetry transformations of the fermions of the theory. These are the gravitino KSE

$$
\nabla_{M} \epsilon_{L}+\frac{1}{4}\left(\partial_{i} \mathcal{K} \mathcal{D}_{M} \phi^{i}-\partial_{i} \mathcal{K} \mathcal{D}_{M} \phi^{\bar{i}}\right) \epsilon_{L}+\frac{i}{2} e^{\frac{\mathcal{K}}{2}} W \Gamma_{M} \epsilon_{R}=0,
$$

the gaugino KSE

$$
F_{M N}^{a} \Gamma^{M N} \epsilon_{L}-2 i \mu^{a} \epsilon_{L}=0,
$$

and the KSE associated with the scalar multiplets

$$
i \Gamma^{M} \epsilon_{R} \mathcal{D}_{M} \phi^{i}-e^{\frac{\mathcal{K}}{2}} G^{\bar{i}} D_{j} \bar{W} \epsilon_{L}=0,
$$

where $\nabla$ is the spin connection, $\phi^{i}$ is a complex scalar field, $\mathcal{K}=\mathcal{K}\left(\phi^{i}, \phi^{\bar{j}}\right)$ is the Kähler potential of the scalar manifold $S$, whose metric is $G_{i j}=\partial_{i} \partial_{j} \mathcal{K}, W=W\left(\phi^{i}\right)$ is a (local) holomorphic function on $S$,

$$
D_{i} W=\partial_{i} W+\partial_{i} \mathcal{K} W, \quad \mathcal{D}_{M} \phi^{i}=\partial_{M} \phi^{i}-A_{M}^{a} \xi_{a}^{i},
$$

$\xi_{a}$ are holomorphic Killing vector fields on $S, A^{a}$ is the gauge connection with field strength $F^{a}$ and $\mu^{a}$ is the moment map, i.e.

$$
G_{i j} \xi_{a}^{\bar{j}}=i \partial_{i} \mu_{a} .
$$

We mostly follow the metric and spinor conventions of [55]. In particular, the spacetime metric has signature mostly plus, $\epsilon$ is a Majorana spinor and $\epsilon_{L, R}=\frac{1}{2}\left(1 \pm \Gamma_{5}\right) \epsilon$, where $\Gamma_{5}^{2}=1$. We have set the gauge coupling to 1 .

The gravitino KSE is a parallel transport equation for a connection which, apart from the Levi-Civita part, contains additional terms that depend on the matter couplings. The gauge group of the KSEs is $\operatorname{Spin}_{c}(3,1)=\operatorname{Spin}(3,1) \times_{\mathbb{Z}_{2}} U(1)$. The $\operatorname{Spin}(3,1)$ part acts on $\epsilon$ with the Majorana representation while $U(1)$ acts on the chiral component $\epsilon_{L}$ with the standard 1-dimensional representation and on the anti-chiral $\epsilon_{R}$ with its conjugate. The additional $U(1)$ gauge transformation is due to the coupling of the spinor $\epsilon$ to the $U(1)$ connection constructed from the Kähler potential $\mathcal{K}$ associated with the matter couplings. In what follows, we use only the $\operatorname{Spin}(3,1)$ component of the gauge group to locally choose the representatives of the Killing spinors.

\subsubsection{Spinors}

We have already described the Dirac spinors of $\operatorname{Spin}(3,1)$ in the context of $\mathcal{N}=2$ theory in Section 3.1.2. Here the difference is that the supersymmetry parameter $\epsilon$ is in the Majorana representation of $\operatorname{Spin}(3,1)$. To impose the reality condition required, let us identify the chiral representation with the even forms, $\Lambda^{\mathrm{ev}}\left(\mathbb{C}^{2}\right)$, and the anti-chiral with odd ones, $\Lambda^{\text {odd }}\left(\mathbb{C}^{2}\right)$. The complex conjugation operation is imposed by the anti-linear map, $r_{A}=-\Gamma_{012} *, r_{A}^{2}=1$, see Appendix B. There 
is one orbit of $\operatorname{Spin}(3,1)=\operatorname{SL}(2, \mathbb{C})$ on $\Lambda^{\mathrm{ev}}\left(\mathbb{C}^{2}\right)-\{0\}$, and so the chiral component of $\epsilon$ can be locally chosen as 1 . Applying $r_{A}$ to the spinor 1 , one finds that a Majorana representative for the orbit is

$$
\epsilon=1+e_{1}, \epsilon_{L}=1, \epsilon_{R}=e_{1} .
$$

This can be chosen as the first Killing spinor of the theory. The isotropy group of the spinor 1 in $\operatorname{SL}(2, \mathbb{C})$ is $\mathbb{C}$. This remaining gauge symmetry will be used later to choose the second Killing spinor.

\section{2. $N=1$ backgrounds}

\subsubsection{Solution of KSES}

Evaluating the KSEs on the first Killing spinor $\epsilon=1+e_{1}$, one finds a linear system which relates some components of the spin connection to the fluxes and matter couplings, and restricts the geometry of the spacetime. The construction of the linear system is similar to that already described for in the minimal $\mathcal{N}=2 d=4$ theory and therefore we shall not give more details. The solution of this linear system for the gravitino KSE gives

$$
\begin{aligned}
& \Omega_{+,+-}=\Omega_{+, 1 \overline{1}}=\Omega_{+,+1}=\Omega_{-,++}=\Omega_{1,+\overline{1}}=\Omega_{1,+1}=0 \\
& \Omega_{-,+1}+\Omega_{1,+-}=0,
\end{aligned}
$$

and

$$
\begin{aligned}
\Omega_{-, 1 \overline{1}}+\frac{1}{2}\left(\partial_{i} \mathcal{K} \mathcal{D}_{-} \phi^{i}-\partial_{\bar{i}} \mathcal{K} \mathcal{D}_{-} \phi^{\bar{i}}\right) & =0, \\
i \sqrt{2} e^{\frac{\mathcal{K}}{2}} W+2 \Omega_{-,+\overline{1}} & =0, \\
\Omega_{-,+1}+\Omega_{1,1 \overline{1}}+\frac{1}{2}\left(\partial_{i} \mathcal{K} \mathcal{D}_{1} \phi^{i}-\partial_{\bar{i}} \mathcal{K} \mathcal{D}_{1} \phi^{\bar{i}}\right) & =0 .
\end{aligned}
$$

The conditions in (61) are considered as restrictions on the geometry of spacetime while the conditions in (62) are thought of as an expression of the fluxes in terms of the geometry.

Similarly, the solution of the linear system for the gaugino (56) and the matter multiplet (57) KSEs gives

$$
F_{+1}^{a}=F_{+-}^{a}=0, \quad F_{1 \overline{1}}^{a}-i \mu^{a}=0,
$$

and

$$
\mathcal{D}_{+} \phi^{i}=0, \quad \sqrt{2} i \mathcal{D}_{1} \phi^{i}=e^{\frac{\mathcal{K}}{2}} G^{\bar{j}} D_{j} \bar{W},
$$

respectively. In what follows, we explore the consequences of the above conditions on the geometry of spacetime.

\subsubsection{Geometry}

To proceed, the metric in the spinorial geometry coframe is

$$
d s^{2}=2 \mathbf{e}^{-} \mathbf{e}^{+}+2 \mathbf{e}^{1} \mathbf{e}^{\overline{1}} .
$$

The form bilinears associated with the Killing spinor $\epsilon$ are

$$
X=\mathbf{e}^{-}, \quad \tau=\mathbf{e}^{-} \wedge\left(\mathbf{e}^{1}+\mathbf{e}^{\overline{1}}\right),
$$

and their spacetime duals. Observe that $X$ is invariant under the $U(1)$ transformations generated by the Kähler potential, while $\tau$ is not, and thus it is a section of a $U(1)$ bundle. The conditions on the geometry (61) can now be re-written as

$$
\mathcal{L}_{X} g=0, \quad \mathbf{e}^{-} \wedge d \mathbf{e}^{-}=0, \quad \mathbf{e}^{-} \wedge \mathbf{e}^{\overline{1}} \wedge d \mathbf{e}^{1}=0 .
$$

Observe also that $\mathbf{e}^{-} \wedge \mathbf{e}^{1} \wedge d \mathbf{e}^{1}=0$ and $\mathcal{L}_{X} \epsilon=0$, where $\mathcal{L}_{X}$ is the spinorial Lie derivative (38).

The first condition in (67) implies that the metric admits a null Killing vector field. While the second implies that the distribution defined by $X$ is integrable. Therefore, there is locally a function $h$ such that $\mathbf{e}^{-}=h d v$ for some coordinate $v$. Adapting also a coordinate along $X, X=\partial_{u}$, and after a coframe rotation as in (50), the metric can be written as in (65) with

$$
\mathbf{e}^{-}=h d v, \quad \mathbf{e}^{+}=d u+V d v+w_{I} d x^{I}, \quad \mathbf{e}^{1}=\beta_{I} d x^{I},
$$

where $u, v, x^{I}, I=1,2$, are real coordinates, and $h, V, w_{I}$ are real and $\beta_{1}, \beta_{2}$ are complex spacetime functions, respectively. As the metric and the form bilinears are invariant under the action of $X$, the coframe above can be chosen to be $u$ independent. 
The conditions that relate the fluxes to the geometry in (62) can be rewritten in terms of the form bilinears $X$ and $\tau$ as

$$
\begin{aligned}
& \frac{1}{2}\left(\partial_{i} \mathcal{K} \mathcal{D}_{-} \phi^{i}-\partial_{\bar{i}} \mathcal{K} \mathcal{D}_{-} \phi^{\bar{i}}\right)+\nabla_{-} \tau_{-1}=0, \\
& \begin{aligned}
\sqrt{2} e^{\frac{\mathcal{K}}{2}} W \mathbf{e}^{-}-\star\left(\mathbf{e}^{1} \wedge d \mathbf{e}^{-}\right) & =0, \\
\star d\left(\mathbf{e}^{-} \wedge \mathbf{e}^{\overline{1}}\right)-\frac{1}{\sqrt{2}} e^{\frac{\mathcal{K}}{2}} \bar{W} \mathbf{e}^{-}-\frac{i}{2}\left(\partial_{i} \mathcal{K} \mathcal{D}_{1} \phi^{i}-\partial_{i} \mathcal{K} \mathcal{D}_{1} \phi^{\bar{i}}\right) \mathbf{e}^{-} & =0,
\end{aligned}
\end{aligned}
$$

where the orientation of the spacetime is chosen as $\epsilon_{-+1 \overline{1}}=-i$.

To solve (63), one can locally always choose the gauge $A_{+}^{a}=0$. The first two conditions in (63) will then imply that the remaining components of $A$ are independent of $u$. There is no general procedure to give an explicit solution for the last condition (63).

Next we turn to the conditions (64) that arise from the KSEs of the matter multiplet. In the gauge $A_{+}^{a}=0$, the first condition in (64) implies that the scalar fields can be taken to be independent of $u, \partial_{u} \phi=0$. The last condition in (64) can be interpreted as a holomorphic flow equation. The construction of explicit solutions will depend on the form of the Kähler potential and $W$, i.e. on the details of the model. This concludes the solution of the KSEs for one Killing spinor of $\mathcal{N}=1$ $d=4$ supergravity coupled to any number of vector and scalar multiplets.

\section{3. $N=2$ backgrounds}

\subsubsection{Killing spinors}

The first Killing spinor is the same as that of the $N=1$ case investigated above. So we set $\epsilon_{1}=\epsilon$, where $\epsilon$ is given in (60). To choose the second Killing spinor, consider the most general Majorana spinor

$$
\epsilon_{2}=a 1+b e_{12}+r_{A}\left(a 1+b e_{12}\right), \quad a, b \in \mathbb{C} .
$$

The isotropy group of $\epsilon_{1}$ in $\operatorname{Spin}(3,1)$ is $\mathbb{C}$. This can be used to simplify the expression for $\epsilon_{2}$. There are two cases to consider. First if $b=0$, the $\mathbb{C}$ isotropy transformations leave $\epsilon_{2}$ invariant. Therefore, one can set

$$
\epsilon_{2}=a 1+\bar{a} e_{1} .
$$

Linear independence of $\epsilon_{1}$ and $\epsilon_{2}$ requires that $\operatorname{Im} a \neq 0$.

Next suppose that $b \neq 0$. Acting on $\epsilon_{2}$ with the isotropy group $\mathbb{C}$ of the first Killing spinor with parameter $\lambda$, one has

$$
\epsilon_{2}^{\prime}=(a+\lambda b) 1+b e_{12}+r_{A}\left[(a+\lambda b) 1+b e_{12}\right] .
$$

Setting $\lambda=-\frac{a}{b}$, one can choose a normal form for $\epsilon_{2}$ as

$$
\epsilon_{2}=b e_{12}-\bar{b} e_{2} \text {. }
$$

So the second Killing spinor $\epsilon_{2}$ can be locally chosen either as in (71) or as in (73) with $a, b$ promoted to complex spacetime functions.

\subsubsection{Solution of KSEs for $\epsilon_{2}=a 1+\bar{a} e_{1}$}

Consider first the case for which $\epsilon_{2}=a 1+\bar{a} e_{1}$. The linear system is easy to read off from that of the $N=1$ case. In particular, the supercovariant connection along the - light-cone direction gives

$$
2 a \Omega_{-,+\overline{1}}+i \sqrt{2} \bar{a} e^{\frac{\mathcal{K}}{2}} W=0 .
$$

Comparing this condition with those of the $N=1$ case in (62), one concludes that either $W=0$ on the field configurations $\phi$ of the solution or $a=\bar{a}$. If the latter is the case, then it turns out that $a$ is also constant and so $\epsilon_{2}$ is not linearly independent of $\epsilon_{1}$.

Therefore, for these $N=2$ solutions, we have to choose $W=0$. After some further investigation of the gravitino and scalar multiplets KSEs, we find that the conditions for $N=2$ supersymmetry are

$$
\Omega_{A,+B}=\Omega_{+, 1 \overline{1}}=0, \quad \partial_{A} a=0,
$$

and

$$
\begin{array}{r}
\Omega_{-, 1 \overline{1}}+\frac{1}{2}\left(\partial_{i} \mathcal{K} \mathcal{D}_{-} \phi^{i}-\partial_{\bar{i}} \mathcal{K} \mathcal{D}_{-} \phi^{\bar{i}}\right)=0, \quad \Omega_{1,1 \overline{1}}-\frac{1}{2} \partial_{\bar{i}} \mathcal{K} \mathcal{D}_{1} \phi^{\bar{i}}=0, \\
W=\partial_{j} W=0, \quad \mathcal{D}_{1} \phi^{i}=\mathcal{D}_{+} \phi^{i}=0 .
\end{array}
$$

Therefore $a$ is constant. There are no additional conditions that arise from the gaugino Killing spinor equation apart from those that we have found in the $N=1$ case (63). 
4.3.3. Solution of KSEs for $\epsilon_{2}=b e_{12}-\bar{b} e_{2}$

A direct substitution of $\epsilon_{2}=b e_{12}-\bar{b} e_{2}$ into the gravitino KSE reveals that

$$
\begin{aligned}
\partial_{+} b=0, \quad b \Omega_{+,-1}+\bar{b} \Omega_{-,+\overline{1}} & =0, \\
\partial_{-} b-\Omega_{-, 1 \overline{1}} b=0, & \Omega_{-,-1}=0, \\
\partial_{1} b-b\left(\Omega_{1,-+}+\Omega_{+,-1}+\Omega_{1,1 \overline{1}}\right)=0, & \Omega_{1,-1}=0, \\
\partial_{\overline{1}} b-b \Omega_{\overline{1}, 1 \overline{1}}=0, & \Omega_{\overline{1},-1}=0,
\end{aligned}
$$

where we have used the $N=1$ relations in (61) and (62) to simplify the expressions. Similarly, the gaugino KSE gives

$$
F_{-1}^{a}=0, \quad F_{1 \overline{1}}^{a}+i \mu^{a}=0 .
$$

Furthermore, the KSEs associated with the matter multiplets evaluated on $\epsilon_{2}$ reveal that

$$
\mathcal{D}_{-} \phi^{i}=0, \quad i \sqrt{2} \bar{b} \mathcal{D}_{\overline{1}} \phi^{i}+b e^{\frac{\mathcal{K}}{2}} G^{i \bar{j}} D_{\bar{j}} \bar{W}=0 .
$$

One can easily combine the above conditions with those described in (61)-(64) which arise from demanding that $\epsilon_{1}$ is a Killing spinor. Below we shall describe the consequences that all these conditions have on the spacetime geometry and the restrictions they impose on the fields.

\subsubsection{Geometry of $N=2$ backgrounds}

Case 1: $\epsilon_{2}=a 1+\bar{a} e_{1}$

The geometric conditions (75) imply that

$$
\nabla_{A} X_{B}=0, \quad \mathbf{e}^{-} \wedge \mathbf{e}^{\overline{1}} \wedge d \mathbf{e}^{1}=0,
$$

where $X=\mathbf{e}^{-}$. Thus the spacetime admits a null parallel vector field $X$. Note also that as a consequence of the above conditions $\mathcal{L}_{X} \epsilon_{1}=\mathcal{L}_{X} \epsilon_{2}=0$, where $\mathcal{L}_{X}$ is the spinorial Lie derivative defined in (38).

Apart from $X$, the spacetime admits a 2-form bilinear $\rho=\mathbf{e}^{-} \wedge \mathbf{e}^{1}$. Observe that $\tau$ in (66) is the real part of $\rho$. The investigation of the conditions in (76) is similar to those in (62) and (64) in the $N=1$ case. In particular, we have

$$
\begin{aligned}
\frac{1}{2}\left(\partial_{i} \mathcal{K} \mathcal{D}_{-} \phi^{i}-\partial_{\bar{i}} \mathcal{K} \mathcal{D}_{-} \phi^{\bar{i}}\right)+\nabla_{-} \tau_{-1} & =0, \\
\star d\left(\mathbf{e}^{-} \wedge \mathbf{e}^{\overline{1}}\right)+\frac{i}{2} \partial_{\bar{i}} \mathcal{K} \mathcal{D}_{1} \phi^{\bar{i}} \mathbf{e}^{-} & =0, \\
W=\partial_{j} W=0, \quad \mathcal{D}_{1} \phi^{i}=\mathcal{D}_{+} \phi^{i} & =0 .
\end{aligned}
$$

It is apparent from this that the scalar fields must lie on both the vanishing locus and critical points of $W$. Furthermore, they obey a light-cone holomorphicity condition as a consequence of the last two conditions in the above equation. Observe that the distribution spanned by $\left(\partial_{+}, \partial_{1}\right)$ is integrable as $d \mathbf{e}^{-}=0$, and $d \mathbf{e}^{\overline{1}}\left(\partial_{+}, \partial_{1}\right)=0$. A coframe can be chosen as in (68) but now with $h=1$. Further simplifications are possible in special gauges. For example one can choose $A_{+}^{a}=A_{1}^{a}=0$ as $F_{+1}=0$. For more details on the geometry of these solutions see [53].

The physical interpretation of spacetimes with a null parallel Killing vector field is that of a pp-wave. However this class also includes the cosmic string solutions [56] and their generalizations [57,58].

Case $2: \epsilon_{2}=b e_{12}-\bar{b} e_{2}$

To analyse the conditions (77) which arise from the KSEs, it is convenient to define the 1 -forms

$$
X=\mathbf{e}^{-}, \quad Y=|b|^{2} \mathbf{e}^{+}, \quad Z=\bar{b} \mathbf{e}^{1}+b \mathbf{e}^{\overline{1}}, \quad V=i \bar{b} \mathbf{e}^{1}-i b \mathbf{e}^{\overline{1}} .
$$

Observe that $Z$ is orthogonal to $X, Y, V$, and $V$ is orthogonal to $X, Y, Z$. Then it is straightforward to show that the Killing spinor equations imply that $X, Y$ and $Z$ are all Killing vectors. Furthermore, $V$ is closed, $d V=0$. In addition, one finds the following commutators

$$
[V, X]=[V, Y]=[V, Z]=0,
$$

and

$$
[X, Y]=c Z, \quad[X, Z]=-2 c K, \quad[Y, Z]=2 c Y,
$$

where $c=b\left(\Omega_{-,+1}-\Omega_{+,-1}\right)$.

Consider the commutator $[X, Y]=c Z$. Since $V$ commutes with the other three vector field, the Jacobi identity implies that $V c=0$. Similarly, the Jacobi identity for $Z, X$ and $Y$ together with the linear independence of these three vector field imply that $X c=Y c=Z c=0$. So $c$ can be taken to be a constant.

Next, since $Z$ and $V$ commute one can choose coordinates $x, y$ such that $Z=\partial_{x}$ and $V=\partial_{y}$. Moreover, the rest of the commutators imply that there are additional coordinates $u, v$ such that

$$
X=e^{2 c x} \partial_{u}, \quad Y=e^{-2 c x}\left(c^{2} u^{2} \partial_{u}+c u \partial_{x}+\partial_{v}\right) .
$$


Using (82), one can compute the coframe in terms of the coordinates $x, y, v, u$ to find

$$
\begin{aligned}
& \mathbf{e}^{-}=e^{2 c x}|b|^{2} d v, \quad \mathbf{e}^{+}=e^{-2 c x}\left(d u-c^{2} u^{2} d v\right), \\
& \mathbf{e}^{1}=b[(d x-i d y)-c u d v], \quad \mathbf{e}^{\overline{1}}=\bar{b}[(d x+i d y)-c u d v] .
\end{aligned}
$$

Hence the spacetime metric can be written as

$$
d s^{2}=2|b|^{2}\left[d s^{2}\left(M_{3}\right)+d y^{2}\right],
$$

where

$$
d s^{2}\left(M_{3}\right)=d v\left(d u-c^{2} u^{2} d v\right)+(d x-c u d v)^{2} .
$$

Thus $M_{3}$ is either $\mathbb{R}^{2,1}$ if $c=0$, or $A d S_{3}$ if $c \neq 0$.

The function $b$ depends only on $y$. After some computation, one finds that

$$
\frac{d b}{d y}=\sqrt{2}|b|^{2} e^{\frac{\mathcal{K}}{2}} W+\frac{1}{\sqrt{2}} e^{\frac{\mathcal{K}}{2}} b\left(b \partial_{i} \mathcal{K} G^{i j} D_{j} \bar{W}-\bar{b} \partial_{i} \mathcal{K} G^{i j} D_{j} W\right) .
$$

which relates the only unknown component of the metric to the scalar fields.

Combining the conditions that arise from gaugino KSE on both $\epsilon_{1}$ and $\epsilon_{2}$ spinors, one finds that

$$
F^{a}=0, \quad \mu^{a}=0 .
$$

So the gauge connection is flat and can locally be set to zero. The vanishing of the moment map restricts the scalars to lie on a Kähler quotient $S / / H$ of $S$, where $H$ is the gauge group.

Setting $A=0$ locally, the conditions on $\mathcal{D} \phi^{i}$ imply that $\partial_{u} \phi^{i}=\partial_{x} \phi^{i}=\partial_{v} \phi^{i}=0$. Moreover, the remaining Killing spinor equations of the scalar multiplet (79) gives

$$
\frac{d \phi^{i}}{d y}=-\sqrt{2} b e^{\frac{\mathcal{K}}{2}} G^{\bar{i}} D_{j} \bar{W} .
$$

Observe that this expression depends on $b$. This is again a flow equation driven by the holomorphic potential $W$. One can change parameterization to simplify the flow equations (89) and (91). The construction of explicit solutions depends on the details of the models.

Clearly, the spacetime is of cohomogeneity one with a homogeneous section either $A d S_{3}$ or $\mathbb{R}^{2,1}$. So this class of $N=2$ solutions can be thought of as domain wall spacetimes. For a review of the domains walls in supergravity theories as well as their applications see [59].

\section{4. $N=3$ and $N=4$ backgrounds}

\subsubsection{Killing spinors for $N=3$ backgrounds}

Let us first consider the $N=3$ backgrounds. It is clear that after choosing the first two Killing spinors using the $\operatorname{Spin}(3,1)$ covariance of the theory, there is little or no more gauge symmetry left to restrict the choice of the third Killing spinor. This could potentially lead to difficulties with solving the KSEs. Because of this, we use instead a technique which was originally applied to classify the near maximally supersymmetric backgrounds of IIB supergravity in [60]. As at each point in spacetime, the three Killing spinors span a hyperplane in the space of Majorana spinors, we use the $\operatorname{Spin}(3,1)$ gauge symmetry of the theory to restrict the form of the normal $v$ to the hyper-plane of the Killing spinors. As $\operatorname{Spin}(3,1)$ has a single non-trivial orbit on the space of Majorana spinors, we can always chose $v=i\left(e_{2}+e_{12}\right)$. The orthogonal directions to $v$ with respect to Majorana inner product, $A(\zeta, \eta)=\left\langle\Gamma_{12} \zeta^{*}, \eta\right\rangle$, see Appendix B, are $\left\{\eta_{r}\right\}=\left\{1+e_{1}, e_{2}-e_{12}, i\left(e_{2}+e_{12}\right)\right\}$. So the three Killing spinors can be chosen as

$$
\epsilon_{r}=\sum_{s} f_{r s} \eta_{s}, \quad r, s=1,2,3,
$$

where $f_{r s}$ is a real $3 \times 3$ invertible matrix of spacetime functions. Schematically we write $\epsilon=f \eta$.

In $N=4$ backgrounds, the Killing spinors can again be written as a linear combination of the basis $\left\{1+e_{1}, i\left(1-e_{1}\right), e_{2}-\right.$ $\left.e_{12}, i\left(e_{2}+e_{12}\right)\right\}$ of Majorana spinors with real spacetime functions as coefficients. Next we shall solve the Killing spinor equations for both cases.

\subsubsection{Local $N=3$ supersymmetry implies $N=4$}

Let us begin with the $N=3$ case. We shall first solve the Killing spinor equations locally. To proceed, observe that (92) implies that schematically $\epsilon_{L}=f \eta_{L}$ and $\epsilon_{R}=f \eta_{R}$. Substituting this into the gaugino (56) and scalar multiplet (57) KSEs, one finds that the dependence on $f$ can be eliminated, because $f$ is invertible. Moreover the conditions that one obtains are those of (63) and (64), or (78) and (79) for $b=1$ or $b=i$, respectively. These imply that

$$
F_{M N}^{a}=\mathcal{D}_{M} \phi^{i}=D_{i} W=\mu^{a}=0 .
$$


Since the gauge connection is flat, we can locally set the gauge potential to vanish, $A_{M}^{a}=0$. As a result the second equation implies that $\phi^{i}$ are constant. Substituting these data into the gravitino Killing spinor equation, and computing its integrability condition, we obtain

$$
R_{M N, R S} \Gamma^{R S} \eta_{L}+2 e^{\mathcal{K}} W \bar{W} \Gamma_{M N} \eta_{L}=0 .
$$

Clearly the integrability condition takes values in $\mathfrak{s p i n}(3,1)$. Since the isotropy group of three linearly independent spinors in $\operatorname{Spin}(3,1)$ is the identity, (94) implies that

$$
R_{M N, R S}=-e^{\mathcal{K}} W \bar{W}\left(g_{M R} g_{N S}-g_{M S} g_{N R}\right) .
$$

It is easy to see that (93) and (95) are precisely the conditions that one finds for backgrounds that admit maximal $N=4$ supersymmetry. So one concludes that $N=3$ backgrounds locally admit an additional Killing spinor and are therefore locally maximally supersymmetric. Furthermore, (95) implies that the spacetime is either $\mathbb{R}^{3,1}$ or $A d S_{4}$. In the former case, $e^{\mathcal{K}}|W|^{2}=0$ and in the latter $e^{\mathcal{K}}|W|^{2} \neq 0$ when evaluated at the constant maps $\phi$, respectively.

The moment map condition in (93), $\mu^{a}=0$, together with the remaining constant gauge transformations imply that the constant maps $\phi$ take values in a Kähler quotient $S / / H$ of the scalar manifold $S$. It remains to investigate $D_{i} W=0$. Suppose that we have chosen some constant maps $\phi=\phi_{0}$. If $W\left(\phi_{0}\right)=0$, then $D_{i} W=0$ implies that $\partial_{i} W\left(\phi_{0}\right)=0$. So $W$ and its first derivative vanish at $\phi=\phi_{0}$. On the other hand if $W\left(\phi_{0}\right) \neq 0, D_{i} W=0$ relates the value of the first derivative of $W$ to that of the Kähler potential at $\phi=\phi_{0}$.

The physical interpretation of the $N=4$ backgrounds is that they are the supersymmetric vacua of the supergravity theory. The spacetime geometry is either Minkowski or $A_{d} S_{4}$.

Although the existence of local geometries which preserve strictly $N=3$ supersymmetry has been ruled out, the possibility still remains that such backgrounds can be constructed as discrete quotients of maximally supersymmetric ones. We shall not give the details here. This question has been raised in [61] in the context of $\mathcal{N}=2$ supergravity theory. One can show that a background that preserves strictly $N=3$ supersymmetries can be constructed as discrete quotient of a maximally supersymmetric $A d S_{4}$ solution [62].

\subsection{A reflection on the results}

One of the conclusions from the results presented is that there is a systematic way to find the solutions of the KSEs of $\mathcal{N}=1 d=4$ supergravity coupled to any number of vector and scalar multiplets. Although this is not sufficient to find all supersymmetric solutions of the theory as for those the field equations have to be solved as well, a narrative emerges regarding the geometry of the solutions. This is especially apparent for those that preserve $N>1$ supersymmetry as such backgrounds are sufficiently constrained. As a result, their physical interpretation is more apparent and can be extracted from their geometric properties.

The observations made here regarding the geometry of supersymmetric backgrounds extend to other supersymmetric theories. A more involved example is the solution of the KSEs of heterotic supergravity. As for the $\mathcal{N}=1 d=4$ supergravity, the geometry of all supersymmetric heterotic backgrounds can be identified. This leads to the categorization of all the supersymmetric solutions of the theory that have been found as well as to new directions that remain to be investigated.

\section{Minimal $\mathcal{N}=1 d=5$ supergravity}

The bosonic fields of the theory are a metric and a $U(1)$ gauge field $A$ with field strength $F=d A$. The action of the theory is the Einstein-Maxwell system with the addition of a Chern-Simons term for the $U(1)$ field. This theory has found widespread applications in the microscopic counting of black hole entropy within string theory [3]. This is because some brane configurations of 10- and 11-dimensional supergravities dimensionally reduce to black hole solutions of this theory and brane techniques in string theory can be used to do the counting.

Minimal $\mathcal{N}=1 d=5$ supergravity is the first theory whose KSE was systematically solved using the bilinears method [8]. Here we shall present the solution of the KSE employing both the bilinears and spinorial geometry methods to provide a comprehensive example for both methods used to solve KSEs.

\subsection{KSE and field equations}

The only fermion of the theory is the gravitino whose supersymmetry variation leads to the KSE

$$
\mathcal{D}_{A} \epsilon=0,
$$

where the supercovariant connection is

$$
\mathcal{D}_{A}:=\nabla_{A}-\frac{i}{4 \sqrt{3}}\left(\Gamma_{A}^{B C}-4 \delta_{A}^{B} \Gamma^{C}\right) F_{B C},
$$


and $\epsilon$ is in the Dirac representation of $\operatorname{Spin}(4,1)$. The supercovariant curvature can be written as

$$
\begin{aligned}
\mathcal{R}_{A B}= & \frac{1}{4} \hat{R}_{A B, C D} \Gamma^{C D}+\frac{i}{\sqrt{3}}\left(\hat{\nabla}_{A} F_{B C}-\hat{\nabla}_{B} F_{A C}\right) \Gamma^{C} \\
& +\frac{2 i}{3} H_{A B}{ }^{D} F_{D C} \Gamma^{C}-\frac{2}{3} F_{A C} F_{B D} \Gamma^{C D},
\end{aligned}
$$

where $\hat{R}$ is the curvature of the connection $\hat{\nabla}_{A} Y^{B}:=\nabla_{A} Y^{B}+(1 / \sqrt{3}) H^{B}{ }_{A C} Y^{C}$ and $H=* F$ with $\epsilon^{01234}=1$.

Furthermore, a straightforward computation using $d F=0$ reveals that

$$
\Gamma^{B} \mathcal{R}_{A B}=-\frac{1}{2} E_{A B} \Gamma^{B}-\frac{1}{12 \sqrt{3}} L F_{A B_{1} B_{2} B_{3}} \Gamma^{B_{1} B_{2} B_{3}}+\frac{i}{\sqrt{3}} * L F_{A},
$$

where

$$
\begin{aligned}
E_{A B} & :=R_{A B}-2\left(F_{A C} F_{B}{ }^{C}-\frac{1}{6} g_{A B} F^{2}\right)=0, \\
L F & :=d^{*} F-\frac{2}{\sqrt{3}} F \wedge F=0,
\end{aligned}
$$

are the field equations of the theory .

It turns out that as in the minimal $\mathcal{N}=2 d=4$ supergravity, the KSE (96) admits either four or eight Killing spinors. To see this, first observe that (96) is linear over the complex numbers so if $\epsilon$ is a Killing spinor so is $i \epsilon$. Moreover, if $\epsilon$ is a Killing spinor so is $r_{\mathrm{A}} \epsilon$, where $r_{\mathrm{A}}=\Gamma_{12} *$, see Appendix B for the definition and properties of these Clifford algebra operations. As $\epsilon$, $i \epsilon, r_{\mathrm{A}} \epsilon$ and $i r_{\mathrm{A}} \epsilon$ are linearly independent, the solutions of (96) come as multiples of four.

\subsection{Solution of the KSE using the bilinears method}

To begin the analysis of the KSE using the bilinears method [8], we use Fierz identities to obtain algebraic conditions on various form bilinears. Then further conditions on the geometry and flux are determined by an analysis of the KSE. To start, we define a real scalar $f$ and a real form bilinear $X$ by

$$
\text { if } f^{2}:=D(\epsilon, \epsilon), \quad X:=D\left(\epsilon, \Gamma_{A} \epsilon\right) \mathbf{e}^{A} .
$$

Note that the bilinear $X$ cannot vanish identically, as by definition $X_{0}=\left\|\Gamma_{0} \epsilon\right\|^{2}=\|\epsilon\|^{2} \neq 0$. We have also chosen a convention for which $D(\epsilon, \epsilon)=i f^{2}$. If a spinor $\hat{\epsilon}$ satisfies $D(\hat{\epsilon}, \hat{\epsilon})=-i f^{2}$, then there exists a spin transformation, which lies in a disconnected component of the spin group, relating $\hat{\epsilon}$ to a spinor $\epsilon$ satisfying $D(\epsilon, \epsilon)=i f^{2}$. Hence, without loss of generality, we take $D(\epsilon, \epsilon)=i f^{2}$.

Further 2 -form bilinears $\omega_{1}$ and $\xi$ are given by

$$
\omega_{1}:=\frac{1}{2} D\left(\epsilon, \Gamma_{A B} \epsilon\right) \mathbf{e}^{A} \wedge \mathbf{e}^{B}, \quad \xi:=\frac{1}{2} D\left(\epsilon, \Gamma_{A B} r_{A} \epsilon\right) \mathbf{e}^{A} \wedge \mathbf{e}^{B},
$$

where $\omega_{1}$ is real and $\xi$ is complex. There are no other non-vanishing scalar or 1-form bilinears.

The bilinears satisfy a number of algebraic conditions due to the Fierz identities (D.1) and (D.2), which are derived in detail in Appendix D. On defining $\xi=\omega_{2}+i \omega_{3}$, for real 2-forms $\omega_{2}, \omega_{3}$, these algebraic conditions on the bilinears can be rewritten as

$$
\begin{aligned}
& i_{X} \omega_{r}=0, \quad i_{X}{ }^{*} \omega_{r}=-f^{2} \omega_{r}, \quad \omega_{r} \wedge \omega_{s}=-2 f^{2} \delta_{r s}{ }^{*} X, \\
& \left(\omega_{r}\right)_{C A}\left(\omega_{S}\right)_{B}{ }_{B}=\delta_{r s}\left(f^{4} g_{A B}+X_{A} X_{B}\right)+\epsilon_{r s}^{p} f^{2}\left(\omega_{p}\right)_{A B},
\end{aligned}
$$

and

$$
X^{2}=-f^{4}, \quad r, s, p=1,2,3 .
$$

The spinor $\epsilon$ also satisfies several conditions as a consequence of the Fierz identities, which are

$$
\begin{aligned}
X_{A} \Gamma^{A} \epsilon=i f^{2} \epsilon, \quad\left(\omega_{1}\right)_{A B} \Gamma^{A B} \epsilon=-4 i f^{2} \epsilon, \\
\left(\omega_{2}\right)_{A B} \Gamma^{A B} \epsilon=-4 i f^{2} r_{A} \epsilon, \quad\left(\omega_{3}\right)_{A B} \Gamma^{A B} \epsilon=-4 f^{2} r_{A} \epsilon .
\end{aligned}
$$

Having obtained the algebraic conditions (103)-(105) and (106), the conditions obtained from the Killing spinor equation can be determined. These are 


$$
\begin{aligned}
& d f^{2}=\frac{2}{\sqrt{3}} i_{X} F, \quad \mathcal{L}_{X} g=0, \\
& d X=\frac{4}{\sqrt{3}} f^{2} F+\frac{2}{\sqrt{3}} *(F \wedge X),
\end{aligned}
$$

and

$$
\begin{aligned}
\nabla_{A}\left(\omega_{r}\right)_{B C} & \left.=-\frac{2}{\sqrt{3}} F_{A}^{D}\left({ }^{*} \omega_{r}\right)_{D B C}+\frac{2}{\sqrt{3}} F_{[B}{ }^{D}\left({ }^{*} \omega_{r}\right)_{C}\right]_{A D} \\
& \left.-\frac{1}{\sqrt{3}} g_{A[B}\left({ }^{*} \omega_{r}\right)_{C}\right]^{D_{1} D_{2}} F_{D_{1} D_{2}} .
\end{aligned}
$$

In particular, the first condition in (107) implies that $\mathcal{L}_{X} F=0$, and also $\mathcal{L}_{X} f^{2}=0$. Also, (109) implies that

$$
d \omega_{r}=0
$$

and this together with (103) imply that $\mathcal{L}_{X} \omega_{r}=0$. Therefore the fields $g$ and $F$, as well as the bilinears, are invariant under the action of $X$.

The analysis of these conditions splits into two cases, according as to whether the vector field $X$ is timelike or null, corresponding to the cases $f \neq 0$ and $f=0$ respectively.

\subsubsection{Geometry and supersymmetry of timelike solutions}

In the timelike class of solutions for which $f \neq 0$, it is convenient to introduce a local spacetime coframe $\left\{\mathbf{e}^{0}, \mathbf{e}^{i} ; i=\right.$ $1,2,3,4\}$ such that $X:=f^{2} \mathbf{e}^{0}$.

Adapting a coordinate $t$ along $X, X=\partial_{t}, \mathbf{e}^{0}$ can be written as $\mathbf{e}^{0}=f^{2}(d t+\alpha)$, where $\alpha=\alpha_{i} \mathbf{e}^{i}$. It is also useful to define $\mathbf{e}^{i}:=f^{-1} \dot{\mathbf{e}}^{i}$ in which case the metric becomes

$$
d s^{2}=-f^{4}(d t+\alpha)^{2}+f^{-2} d \dot{s}^{2},
$$

where $d \dot{s}^{2}=\delta_{i j} \mathbf{e}^{i} \mathbf{e}^{\dot{e}}$. The metric $d \dot{s}^{2}$, as well as $f, \alpha, \omega_{r}$ and $F$ are all $t$-independent because as has been mentioned they are invariant under the action of $X$.

Locally the spacetime can be viewed as a fibration over a 4-dimensional base manifold $B$ with fibres the orbits of $X$. The volume form $\operatorname{dvol}_{B}$ on the 4-dimensional base manifold $B$, equipped with metric $d s^{2}$, is related to the 5 -dimensional volume form by dvol ${ }_{5}=f^{-4} \mathbf{e}^{0} \wedge \mathrm{dvol}_{B}$. The conditions (107) and (108) then determine the Maxwell field strength via

$$
F=\frac{\sqrt{3}}{2} d \mathbf{e}^{0}-\frac{1}{\sqrt{3}} f^{2}(d \alpha)_{\text {asd }},
$$

where $(d \alpha)_{\text {asd }}$ denotes the anti-self dual part of $d \alpha$ on $B$.

The base space $B$ admits a hyper-Kähler structure, associated with the three 2-form bilinears $\omega_{r}, r=1,2,3$. To see this, note that $\omega_{r}, r=1,2,3$ descend on $B$ as $\mathcal{L}_{X} \omega_{r}=0$ and $i_{X} \omega_{r}=0$. The 2 -forms $\omega_{r}$ are self-dual on $B$, as a consequence of (103). Moreover the associated complex structures, $I_{r}, \omega_{r}(Y, Z)=\stackrel{g}{g}\left(Y, I_{r} Z\right)$, satisfy the algebra of the imaginary quaternions, $I_{1}^{2}=I_{2}^{2}=-\mathbf{1}, I_{3}=I_{1} I_{2}, I_{1} I_{2}=-I_{2} I_{1}$ on $B$, as a consequence of (104). The remaining content of the condition (109) is

$$
\stackrel{\circ}{\nabla} \omega_{r}=0
$$

where $\nabla^{\nabla}$ denotes the Levi-Civita connection on $B$. Hence the $\omega_{r}$ define a hyper-Kähler structure on $B$.

This analysis exhausts the content of the algebraic and differential conditions which we have obtained on the bilinears. It remains to determine the remaining conditions imposed by the KSEs, given the conditions on the geometry and flux obtained so far. This can be done by noting that the spinor $\epsilon$ must satisfy the condition

$$
\Gamma_{0} \epsilon=-i \epsilon \text {. }
$$

This follows from the first identity in (106). In turn, this implies that $\Lambda_{i j} \Gamma^{i j} \epsilon=0$ for any anti-self-dual 2-form $\Lambda$ on $B$. Then the $M=0$ component of (96) is equivalent to

$$
\partial_{t} \epsilon=0 \text {, }
$$

and the remaining components of (96) are equivalent to

$$
\stackrel{\circ}{\nabla}\left(f^{-1} \epsilon\right)=0 \text {. }
$$

It follows that the spinor $\epsilon$ is given by $\epsilon=f \eta$, where $\eta$ is a covariantly constant $t$-independent spinor on the hyper-Kähler base $B, \Gamma_{0} \eta=-i \eta$. The conditions appearing in (106) which involve $\omega_{r}$ are not involved in the evaluation of (115). Hence any covariantly constant spinor $\eta$ on $B$ satisfying $\Gamma_{0} \eta=-i \eta$ gives rise to a solution $\epsilon=f \eta$ of the KSE of the $d=5$ theory.

This analysis exhausts the content of the KSE. It remains to impose the Bianchi identity and field equations; the resulting conditions are common to both the bilinears method and the spinorial geometry approach to solving the KSE, and will be presented after the spinorial geometry analysis. 


\subsubsection{Geometry and supersymmetry of null solutions}

In the null class of solutions, for which $f=0$, and $X$ is null, it is convenient to introduce a local spacetime coframe $\left\{\mathbf{e}^{-}, \mathbf{e}^{+}, \mathbf{e}^{i}: i=1,2,3\right\}$ such that $X:=\mathbf{e}^{-}$. The algebraic identities (103) then imply that

$$
\omega_{r}=\mathbf{e}^{-} \wedge \tau_{r}
$$

where without loss of generality we take $\left(\tau_{r}\right)_{-}=0$. Furthermore, (104) simplifies to

$$
\left(\omega_{r}\right)_{C A}\left(\omega_{S}\right)^{C}{ }_{B}=\delta_{r s} X_{A} X_{B} .
$$

Setting $s=r, A=-, B=+$ then implies that $\left(\tau_{r}\right)_{+}=0$, and setting $A=B=-$ further implies that

$$
\left(\tau_{r}\right)_{C}\left(\tau_{s}\right)^{C}=\delta_{r s}
$$

Hence we can choose a basis for which $\tau_{r}=\delta_{r i} \mathbf{e}^{i}$. The condition (107) implies that $i_{X} F=0$, so the Maxwell field strength decomposes as

$$
F=F_{-i} \mathbf{e}^{-} \wedge \mathbf{e}^{i}+\frac{1}{2} F_{i j} \mathbf{e}^{i} \wedge \mathbf{e}^{j} .
$$

In order to introduce co-ordinates, we adapt a co-ordinate $u$ such that $X=\partial_{u}$. Also, the algebraic conditions (107) and (108) imply that

$$
X \wedge d X=0,
$$

and it follows that a further local co-ordinate $v$ can be found such that

$$
\mathbf{e}^{-}=h^{-1} d v,
$$

for some function $h$. Next, consider the closure condition (110); this implies that

$$
d v \wedge d\left(h^{-1} \mathbf{e}^{i}\right)=0,
$$

and hence there exist co-ordinates $x^{I}, I=1,2,3$ and functions $p^{I}, I=1,2,3$ such that

$$
\mathbf{e}^{i}=\delta_{I}^{i}\left(h d x^{I}+p^{I} d v\right) .
$$

Using a change of basis as in (50), which leaves $\mathbf{e}^{-}$invariant, one can set without loss of generality $p^{I}=0$, so $\mathbf{e}^{i}=h \delta_{I}^{i} d x^{I}$. The condition $\mathcal{L}_{\partial_{u}} X=0$ implies that $h$ is $u$-independent; and the condition that $X$ is Killing then implies that $\mathcal{L}_{\partial_{u}} \mathbf{e}^{+}=0$. The basis can therefore be written as follows

$$
\mathbf{e}^{+}=d u+V d v+n_{I} d x^{I}, \quad \mathbf{e}^{-}=h^{-1} d v, \quad \mathbf{e}^{i}=h \delta_{I}^{i} d x^{I},
$$

where $V, h, n_{I}$ are $u$-independent. It remains to determine the components $F_{-i}$ and $F_{i j}$ of the flux. To do this, we first write 5-dimensional volume form as $\mathrm{dvol}_{5}=-h^{3} \mathbf{e}^{+} \wedge \mathbf{e}^{-} \wedge \stackrel{\circ}{\epsilon}$, where $\stackrel{\epsilon}{\mathrm{i}}$ is the volume form of the flat 3-metric. The components $F_{i j}$ are determined by the condition (108) as

$$
\frac{1}{2} F_{i j} \mathbf{e}^{i} \wedge \mathbf{e}^{j}=-\frac{\sqrt{3}}{4} \stackrel{\circ}{I J}^{K} \partial_{K} h d x^{I} \wedge d x^{J} .
$$

The remaining components $F_{-i}$ are obtained from the condition (109) on setting $A=M=-, N=j$ to find

$$
F_{-k} \mathbf{e}^{-} \wedge \mathbf{e}^{k}=-\frac{1}{4 \sqrt{3}} \stackrel{\circ}{\epsilon}_{I}^{J K} h^{-2} d n_{J K} d v \wedge d x^{I},
$$

and hence

$$
F=-\frac{1}{4 \sqrt{3}} \dot{\epsilon}_{I}^{J K} h^{-2} d n_{J K} d v \wedge d x^{I}-\frac{\sqrt{3}}{4} \dot{\epsilon}_{I J}{ }^{K} \partial_{K} h d x^{I} \wedge d x^{J} .
$$

On substituting these conditions back into the Killing spinor equation (96), we find that the spinor is constant, $\partial_{M} \epsilon=0$, and as a consequence of the first identity in (106) satisfies $\Gamma_{+} \epsilon=0$.

This analysis exhausts the content of the KSE. It remains to impose the Bianchi identity and field equations. Again, the resulting conditions are common to both the bilinears and the spinorial geometry approaches to solving the KSE, and will be presented after the spinorial geometry analysis.

\subsection{Solution of the KSE using the spinorial geometry method}

One way to describe the Dirac representation of $\operatorname{Spin}(4,1)$ in terms of forms is to begin from that of $\operatorname{Spin}(4)$ and identify $\Gamma^{0}=i \Gamma^{1234}$. Therefore the Dirac spinors of $\operatorname{Spin}(4,1)$ are identified with $\Lambda^{*}\left(\mathbb{C}^{2}\right)$, where the action of the gamma matrices of $\operatorname{Spin}(4)$ on $\Lambda^{*}\left(\mathbb{C}^{2}\right)$ is described in Appendix B. Furthermore $\operatorname{Spin}(4)=S U(2) \times S U(2)$ and acts on $\Lambda^{\text {ev }}\left(\mathbb{C}^{2}\right)$ and $\Lambda^{\text {odd }}\left(\mathbb{C}^{2}\right)$ with the $(\mathbf{2}, \mathbf{1})$ and $(\mathbf{1}, \mathbf{2})$ representations, respectively. As the orbits of $S U(2)$ on $\mathbb{C}^{2}-\{0\}$ are 3 -spheres, a representative of 
the first Killing spinor can be chosen up to a $\operatorname{Spin}(4)$ transformation as $\epsilon=f_{1} 1+f_{2} e_{1}$, where $f_{1}$ and $f_{2}$ are real constants. To further simplify this spinor, consider the $S O(1,1)$ transformation generated by $\Gamma_{03}$. There are three possibilities to consider, according as $\left|f_{1}\right|>\left|f_{2}\right|,\left|f_{1}\right|<\left|f_{2}\right|$ and $\left|f_{1}\right|=\left|f_{2}\right|$. If $\left|f_{1}\right|>\left|f_{2}\right|$, then this transformation can be used to set $f_{2}=0$, whereas if $\left|f_{2}\right|>\left|f_{1}\right|$, then this transformation can be used to set $f_{1}=0$. These two cases, for which either $f_{1}=0$ or $f_{2}=0$, are further related as $\Gamma_{03} e_{1}=-1$, where $\Gamma_{03}$ lies in a disconnected component of the spin group. In the remaining case, $\left|f_{1}\right|=\left|f_{2}\right|$, the $S O(1,1)$ gauge transformations generated by $\Gamma_{03}$ and $\Gamma_{13}$ can be used to set $f_{1}=f_{2}=1$. Therefore, the first Killing spinor can be chosen without loss of generality as

$$
\text { either } \epsilon=f 1 \text {, or } \epsilon=1+e_{1} \text {, }
$$

where $f$ is promoted to a real spacetime function. The isotropy group of $f 1$ and $1+e_{1}$ in $\operatorname{Spin}(4,1)$ is $S U(2)$ and $\mathbb{R}^{3}$, respectively.

\subsection{Case 1: $\epsilon=f 1$}

\subsubsection{Solution of the linear system}

The KSE (96) can be easily evaluated on $f 1$ and after expanding in the basis of Dirac spinors as described in Appendix B, one finds for $A=0, A=\alpha$ and $A=\bar{\alpha}$ the following linear system

$$
\begin{aligned}
& \partial_{0} f+\frac{1}{2} f \Omega_{0, \beta}{ }^{\beta}-\frac{1}{2 \sqrt{3}} f F_{\beta}{ }^{\beta}=0, \quad F_{0 \bar{\beta}}-\frac{\sqrt{3}}{2} \Omega_{0,0 \bar{\beta}}=0, \\
& F_{\alpha \beta}-\sqrt{3} \Omega_{0, \alpha \beta}=0, \quad \partial_{\alpha} f+\frac{1}{2} f \Omega_{\alpha, \beta}{ }^{\beta}+\frac{\sqrt{3}}{2} f F_{0 \alpha}=0, \\
& -\Omega_{\alpha, 0 \bar{\beta}}-\frac{1}{\sqrt{3}} F_{\gamma}^{\gamma} \delta_{\alpha \bar{\beta}}+\sqrt{3} F_{\alpha \bar{\beta}}=0, \quad \Omega_{\alpha, \bar{\beta} \bar{\gamma}}+\frac{2}{\sqrt{3}} \delta_{\alpha[\bar{\beta}} F_{\bar{\gamma}] 0}=0, \\
& \partial_{\bar{\alpha}} f+\frac{1}{2} f \Omega_{\bar{\alpha}, \gamma}^{\gamma}+\frac{1}{2 \sqrt{3}} f F_{0 \bar{\alpha}}=0, \quad-\Omega_{\bar{\alpha}, 0 \bar{\beta}}+\frac{1}{\sqrt{3}} F_{\bar{\alpha} \bar{\beta}}=0, \\
& \Omega_{\bar{\alpha}, \bar{\beta} \bar{\gamma}}=0 .
\end{aligned}
$$

This system can be easily solved to express the flux $F$ in terms of the geometry as

$$
\begin{aligned}
F= & \sqrt{3} d \log f \wedge \mathbf{e}^{0}+\frac{\sqrt{3}}{2} \Omega_{\alpha, 0 \beta} \mathbf{e}^{\alpha} \wedge \mathbf{e}^{\beta}+\frac{\sqrt{3}}{2} \Omega_{\bar{\alpha}, 0 \bar{\beta}} \mathbf{e}^{\bar{\alpha}} \wedge \mathbf{e}^{\bar{\beta}} \\
& +\frac{1}{\sqrt{3}}\left(\Omega_{\alpha, 0 \bar{\beta}}+\delta_{\alpha \bar{\beta}} \Omega_{\gamma, 0} \gamma\right) \mathbf{e}^{\alpha} \wedge \mathbf{e}^{\bar{\beta}},
\end{aligned}
$$

and to find the conditions

$$
\begin{aligned}
& \partial_{0} f=0, \quad \Omega_{\alpha, 0}{ }^{\alpha}-\Omega_{0, \alpha}{ }^{\alpha}=0, \quad \Omega_{0,0 \alpha}=-2 \partial_{\alpha} \log f, \\
& \Omega_{\alpha, \beta}{ }^{\beta}=\partial_{\alpha} \log f, \quad \Omega_{\alpha, 0 \beta}=\Omega_{0, \alpha \beta}, \quad \Omega_{\alpha, 0 \bar{\beta}}+\Omega_{\bar{\beta}, 0 \alpha}=0, \\
& \Omega_{\alpha, \bar{\beta} \bar{\gamma}}=-2 \delta_{\alpha[\bar{\beta}} \partial_{\bar{\gamma}]} \log f, \quad \Omega_{\alpha, \beta \gamma}=0,
\end{aligned}
$$

on the spacetime geometry.

\subsubsection{Geometry}

To investigate the geometry of spacetime, let us note that form bilinears are generated by

$$
\begin{aligned}
& X=D\left(f 1, \Gamma_{A} f 1\right) \mathbf{e}^{A}=f^{2} \mathbf{e}^{0}, \\
& \omega_{1}=\frac{1}{2} D\left(f 1, \Gamma_{A B} f 1\right) \mathbf{e}^{A} \wedge \mathbf{e}^{B}=-i f^{2} \delta_{\alpha \bar{\beta}} \mathbf{e}^{\alpha} \wedge \mathbf{e}^{\bar{\beta}}, \\
& \omega_{2}+i \omega_{3}=\frac{1}{2} D\left(f 1, \Gamma_{A B} i r_{A} f 1\right) \mathbf{e}^{A} \wedge \mathbf{e}^{B}=\frac{1}{2} f^{2} \epsilon_{\alpha \beta} \mathbf{e}^{\alpha} \wedge \mathbf{e}^{\beta},
\end{aligned}
$$

with $\epsilon_{12}=1$. All the geometric conditions in (132) that involve a $\mathbf{e}^{0}$ coframe direction can be expressed as

$$
\mathcal{L}_{X} g=0, \quad \mathcal{L}_{X} \omega_{r}=0, \quad r=1,2,3 .
$$

Therefore $X$ is Killing and leaves the other three 2-form bilinears invariant. In addition, the flux $F$ is also invariant under $X$, $\mathcal{L}_{X} F=0$.

The conditions on the geometry imposed by the remaining three conditions in (132) are

$$
d \omega_{r}=0 .
$$

Therefore the spacetime admits three closed 2-forms. 
Locally the spacetime can be viewed as a fibration with fibres given by the integral curves of $X$. As $i_{X} \omega_{r}=0$ and $\mathcal{L}_{X} \omega_{r}=0$, these forms descend to 2-forms on the base space $B$ of the fibration. As $\omega_{r}$ are closed and Hermitian with respect to the metric $d \mathfrak{s}^{2}=f^{2} \delta_{i j} \mathbf{e}^{i} \mathbf{e}^{j}$ and the associated complex structures $I_{r}, \omega_{r}(Y, Z)=\stackrel{\circ}{g}\left(Y, I_{r} Z\right)$, satisfy the algebra of imaginary unit quaternions, $I_{1}^{2}=I_{2}^{2}=-\mathbf{1}, I_{3}=I_{1} I_{2}, I_{1} I_{2}=-I_{2} I_{1}$ on $B, B$ is a hyper-Kähler manifold. Therefore the spacetime $M$ is a local fibration over a 4-dimensional hyper-Kähler manifold. Notice that $B$ admits a (weak) hyper-Kähler structure with torsion (HKT) [63] with respect to $d \tilde{s}^{2}=\delta_{i j} \mathbf{e}^{i} \mathbf{e}^{j}$ and $f^{-2} \omega_{r}$. As we shall see such structures arise frequently in the investigation of geometries of supersymmetric backgrounds.

These conditions correspond to those obtained via the bilinears method for solving the KSE. Just as in that analysis, special co-ordinates can be found in which the solution can be written in a particularly simple form. In particular, one can adapt a coordinate $t$ such that $X=\partial_{t}$. As all fields and form bilinears are invariant under $X$, a coframe can be chosen on the spacetime which does not depend explicitly on $t$. The remaining decomposition of the metric and Maxwell field strength is identical to the calculation presented in Section 5.2.1.

\subsubsection{Solutions}

The field equations of the theory are implied as a consequence of the KSE, the Bianchi identity of $F, d F=0$ and the vanishing of the electric component of the field equation of $F,{ }^{*} L F_{0}=0$. This follows from the integrability condition of the KSE in (99) which can be rewritten as

$$
-\frac{1}{2} E_{A B} \Gamma^{B} \epsilon+\frac{i}{2 \sqrt{3}}{ }^{*} L F_{B} \Gamma_{A}^{B} \epsilon+\frac{i}{\sqrt{3}} * L F_{A} \epsilon=0 .
$$

As $\epsilon=f 1$, taking the Dirac inner product again with $\epsilon$, one finds that

$$
-\frac{1}{2} E_{A B} X^{A}+\frac{i}{2 \sqrt{3}}{ }^{*} L F_{B}\left(\omega_{1}\right)_{A}^{B}-\frac{1}{\sqrt{3}} f^{2 *} L F_{A}=0 .
$$

where the bilinear $\omega_{1}$ has been defined in (133). For $A=0$, one finds that $E_{00}=0$. On the other hand for $A=i$, one gets that $E_{i 0}=0$ and ${ }^{*} L F_{i}=0$. Therefore, if ${ }^{*} L F_{0}=0$, then the field equation of $F$ will be satisfied. The vanishing of the rest of the components of the Einstein equation follows from an argument similar to that presented in Section 3.2.4 for the minimal $\mathcal{N}=2 d=4$ supergravity.

Therefore to find solutions, one has to solve the Bianchi and the electric component of the field equation for $F$, which in turn gives

$$
d\left(f^{2}(d \alpha)_{\text {asd }}\right)=0, \quad \dot{\nabla}^{2} f^{-2}=\frac{2}{9} f^{4} \stackrel{\circ}{g}^{i j} g^{m n}\left(d \alpha_{\text {asd }}\right)_{i m}\left(d \alpha_{\text {asd }}\right)_{j n},
$$

respectively, where $\nabla^{\circ}$ is the Levi-Civita connection of the hyper-Kähler metric on $B$.

A large class of solutions can be found provided that $d \alpha_{\text {asd }}=0$. In such a case $f^{-2}$ is a harmonic function on a hyper-Kähler manifold $B$. For $B=\mathbb{R}^{4}$ and $f^{-2}=1+\sum_{a} Q_{a} /\left|y-y_{a}\right|^{2}$, the solutions are rotating multi-black holes. The rotation is associated with the self-dual part of $d \alpha$ [64].

Many solutions also arise in the case for which the base space is a Gibbons-Hawking manifold, which admits a triholomorphic isometry [65]. If this tri-holomorphic isometry is a symmetry of the full solution, then the complete solution is determined by a choice of four harmonic functions on $\mathbb{R}^{3}$. To illustrate this construction, we take the base space metric to be

$$
d s^{2}=H^{-1}(d z+\chi)^{2}+H \delta_{r s} d x^{r} d x^{s}, \quad r, s=1,2,3,
$$

where $H$ is a harmonic function on $\mathbb{R}^{3}$ and $\chi=\chi_{r} d x^{r}$ is a 1 -form on $\mathbb{R}^{3}$ satisfying

$$
\star_{3} d \chi=d H \text {. }
$$

The Hodge dual $\star_{3}$ is taken on $\mathbb{R}^{3}$, and the volume form on the base and the volume form on $\mathbb{R}^{3}$ are related by dvol ${ }_{B}=$ $\mathrm{Hdvol}_{3} \wedge \mathrm{dz}$.

These conditions imply that the base metric is hyper-Kähler with tri-holomorphic isometry $\frac{\partial}{\partial z}$. With this base space convention, the hyper-Kähler structure is given by

$$
\omega_{r}=\delta_{r p}(d z+\chi) \wedge d x^{p}-\frac{1}{2} H \epsilon_{r p q} d x^{p} \wedge d x^{q}, \quad r, p, q=1,2,3 .
$$

To construct the solution for which the tri-holomorphic isometry $\frac{\partial}{\partial z}$ is a symmetry of the full solution, decompose $\alpha$ as

$$
\alpha=\Psi(d z+\chi)+\sigma,
$$

where $\Psi$ is a function on $\mathbb{R}^{3}$ and $\sigma$ is a 1 -form on $\mathbb{R}^{3}$. The anti-self-dual part of $d \alpha$ is then

$$
\begin{aligned}
d \alpha_{\text {asd }} & =\frac{1}{2}(d z+\chi) \wedge\left(-d \Psi+H^{-1} \Psi d H+H^{-1} \star_{3} d \sigma\right) \\
& +\frac{1}{2}\left(d \sigma+\Psi \star_{3} d H-H \star_{3} d \Psi\right) .
\end{aligned}
$$


The Bianchi identity from (138) implies that

$$
d\left(f^{2}\left(d \Psi-H^{-1} \Psi d H-H^{-1} \star_{3} d \sigma\right)\right)=0,
$$

and hence there locally exists a function $\rho$ on $\mathbb{R}^{3}$ such that

$$
f^{2}\left(d \Psi-H^{-1} \Psi d H-H^{-1} \star_{3} d \sigma\right)=d \rho .
$$

The remaining content of the Bianchi identity can then be written as

$$
\square_{3}(H \rho)=0,
$$

where $\square_{3}$ denotes the Laplacian on $\mathbb{R}^{3}$. It follows that there exists a harmonic function $K$ on $\mathbb{R}^{3}$ such that

$$
\rho=3 K H^{-1} \text {. }
$$

The gauge field equation given in (138) can then be rewritten as

$$
\square_{3} f^{-2}=\square_{3}\left(K^{2} H^{-1}\right),
$$

so there exists a further harmonic function $L$ on $\mathbb{R}^{3}$ such that

$$
f^{-2}=K^{2} H^{-1}+L \text {. }
$$

Having determined $f$ in terms of these harmonic functions, we determine $\Psi$ by making use of (145), which can be rewritten as

$$
H d \Psi-\Psi d H-\star_{3} d \sigma=3\left(K^{2}+L H\right) d\left(K H^{-1}\right) .
$$

Taking the divergence of this condition gives

$$
\square_{3} \Psi=\square_{3}\left(H^{-2} K^{3}+\frac{3}{2} H^{-1} K L\right),
$$

which implies that there exists a harmonic function $M$ on $\mathbb{R}^{3}$ such that

$$
\Psi=H^{-2} K^{3}+\frac{3}{2} H^{-1} K L+M .
$$

The 1 -form $\sigma$ is then fixed by substituting this expression into (150) to give

$$
\star_{3} d \sigma=H d M-M d H+\frac{3}{2}(K d L-L d K) .
$$

This procedure therefore determines the complete solution entirely in terms of the harmonic functions $\{H, K, L, M\}$; although there is some freedom to redefine these harmonic functions. In particular, the solutions generated by $\{H, K, L, M\}$ and $\left\{H, K^{\prime}, L^{\prime}, M^{\prime}\right\}$ are identical provided that

$$
\begin{aligned}
& K=K^{\prime}+\mu H, \quad L=L^{\prime}-2 \mu K^{\prime}-\mu^{2} H, \\
& M=M^{\prime}+\frac{1}{2} \mu^{3} H-\frac{3}{2} \mu L^{\prime}+\frac{3}{2} \mu^{2} K^{\prime},
\end{aligned}
$$

for constant $\mu$. Also, the harmonic function $M$ is only defined up to an additive constant $v$ with

$$
M=\hat{M}+v, \quad \sigma=\hat{\sigma}-v \chi,
$$

and the harmonic functions $H, K, L$ are unchanged. Furthermore, it is also possible for the same solution to be described by two different Gibbons-Hawking base spaces. For example, the maximally supersymmetric AdS $2 \times S^{3}$ solution can be constructed from both a flat base space, as well as a singular Eguchi-Hanson base.

An example which describes a large family of solutions preserving $N=4$ supersymmetry is given by taking $H=\frac{1}{r}$, so that the base space is $\mathbb{R}^{4}$ together with

$$
\begin{aligned}
& K=-\frac{1}{2} \sum_{i=1}^{P} q_{i} h_{i}, \quad L=1+\frac{1}{4} \sum_{i=1}^{P}\left(Q_{i}-q_{i}^{2}\right) h_{i}, \\
& M=\frac{3}{4} \sum_{i=1}^{P} q_{i}\left(1-\left|\mathbf{y}_{i}\right| h_{i}\right),
\end{aligned}
$$

where $h_{i}=\frac{1}{\left|\mathbf{x}-\mathbf{y}_{i}\right|}$ and $Q_{i}, q_{i}, \mathbf{y}_{i}$ are constant. In the case of a single pole, $P=1$, there are two possibilities. If $\mathbf{y}_{1}=\mathbf{0}$ then the solution will describe a single rotating BMPV black hole, which is static provided that $3 Q_{1}=q_{1}^{2}$. The generic multi-BMPV black hole solution does not however lie within this family of solutions, because although the base space is 
$\mathbb{R}^{4}$, the tri-holomorphic isometry is not a symmetry of the full solution. On the other hand if $\mathbf{y}_{1} \neq \mathbf{0}$, then the solution is the supersymmetric black ring. Further generalization can be made by taking multiple poles. Such solutions include configurations of concentric black rings as well as Black Saturn type of solutions found in [66]. In addition, all of the maximally supersymmetric solutions can be written as solutions in the timelike class with a Gibbons-Hawking base space for which the tri-holomorphic isometry is a symmetry of the solution.

\subsection{Case $2: \epsilon=1+e_{1}$}

\subsubsection{Solution of the linear system}

To find the linear system that arises after evaluating the $\operatorname{KSE}(96)$ on the Killing spinor $1+e_{1}$ observe that $\left(-\Gamma_{0}+\Gamma_{3}\right)(1+$ $\left.e_{1}\right)=0$ in the conventions of Appendix B for $\operatorname{Spin}(4)$ with $\Gamma^{0}=i \Gamma^{1234}$. Because of this it is convenient to change basis to $\left(\Gamma^{+}, \Gamma^{-}, \Gamma^{1}, \Gamma^{2}, \Gamma^{\overline{2}}\right)$ with $\sqrt{2} \Gamma^{ \pm}= \pm \Gamma_{0}+\Gamma_{3}, \Gamma^{1}=e_{1} \wedge+i_{e_{1}}, \Gamma^{2}=\sqrt{2} i_{e_{2}}$ and $\Gamma^{\overline{2}}=\sqrt{2} e_{2} \wedge$. The calculation is similar to the ones we have already presented and thus we shall not elaborate. The solution of the linear system can be written as

$$
F=\frac{1}{2 \sqrt{3}} \epsilon_{i}^{j k} \Omega_{-, j k} \mathbf{e}^{-} \wedge \mathbf{e}^{i}+\frac{\sqrt{3}}{2} \epsilon_{i j}{ }^{k} \Omega_{-,+k} \mathbf{e}^{i} \wedge \mathbf{e}^{j},
$$

where $\epsilon_{12 \overline{2}}=-i$.

The conditions on the geometry are

$$
\begin{array}{lll}
\Omega_{A,+B}+\Omega_{B,+A}=0, & \Omega_{+, i j}=0, \quad \Omega_{i,+j}=0, & \Omega_{2,12}=\Omega_{1,2 \overline{2}}=0, \\
2 \Omega_{-,+2}+\Omega_{1,12}=0, & 2 \Omega_{2,+-}+\Omega_{2,2 \overline{2}}=0, & 2 \Omega_{1,+-}+\Omega_{2,1 \overline{2}}=0 .
\end{array}
$$

This is a full content of the KSE.

\subsubsection{Geometry}

A basis of the form bilinears constructed from the Killing spinors $\epsilon$ and $r_{A} \epsilon$ is

$$
X=\mathbf{e}^{-}, \omega_{r}=\delta_{r i} \mathbf{e}^{-} \wedge \mathbf{e}^{i},
$$

where $\left\{\mathbf{e}^{-}, \mathbf{e}^{+}, \mathbf{e}^{i}: i=1,2,3\right\}$ is the spinorial geometry coframe. The conditions on the geometry (158) can be expressed as

$$
\mathcal{L}_{X} g=0, \quad X \wedge d X=0, \quad d \omega_{r}=0 .
$$

Therefore, $X$ is a Killing vector field which in addition leaves $F$ invariant, $\mathcal{L}_{X} F=0$.

Again, these conditions correspond to those obtained via the bilinears method for solving the KSE, where also special co-ordinates can be adopted. These co-ordinates $u, v$ and $x^{I}$, as well as the decomposition of the metric and Maxwell field strength, are obtained in exactly the same was as described in Section 5.2.2.

\subsubsection{Solutions}

An investigation of the integrability conditions (99) reveals that all field equations are satisfied provided that the $E_{--}$and ${ }^{*} L F_{-}$components of the field equations vanish together with $d F=0$. The latter implies that

$$
\delta^{I J} \partial_{I} \partial_{J} h=0, \quad \partial_{v} \partial_{I} h=-\frac{1}{3} \delta^{J K} \partial_{J}\left(d n_{K I} h^{-2}\right) .
$$

The Einstein equation $E_{--}=0$ gives

$$
\begin{aligned}
& h^{-3} \delta^{I J} \partial_{I}\left(-\partial_{J} V h+\partial_{v} n_{J}\right)-3 h \partial_{v}^{2} h-3\left(\partial_{v} h\right)^{2}+\frac{3}{2} \delta^{I J}\left(\partial_{I} V \partial_{J} h\right. \\
& \left.-\partial_{v} n_{I} h^{-2} \partial_{J} h\right)+\frac{1}{6} \delta^{I J} \delta^{K L} d n_{I K} d n_{J L}=0,
\end{aligned}
$$

and ${ }^{*} L F_{-}=0$ is satisfied with no further conditions.

These equations can be solved and solutions include a magnetic multi-string solution for $V=n=0$ and $h=$ $1+\sum_{a} Q_{a} /\left|x-x_{a}\right|$ and a multi pp-wave solution for $h=1, n=0$ and $V=\sum_{a} Q_{a} /\left|x-x_{a}\right|$.

\subsection{Maximally supersymmetric backgrounds}

The supercovariant curvature (98) of maximally supersymmetric backgrounds vanishes. It is a consequence of the homogeneity theorem [67,68], which will be demonstrated in Section 10.4, that the maximally supersymmetric solutions must be homogeneous spaces. Upon using the Bianchi identity $d F=0$, one can establish from the term linear in gamma matrices in the supercovariant curvature that

$$
\left.\check{\nabla}_{A} F_{B C}=0, \quad H^{D}{ }_{A B} F_{C}\right]_{D}=0,
$$


where $\check{\nabla}=\nabla-(1 / \sqrt{3}) H$. The latter relation also follows from $H={ }^{*} F$. The quadratic term in gamma matrices in the supercovariant curvature gives that

$$
\check{R}_{A B, C D}=\frac{4}{3} F_{A B} F_{C D} .
$$

Therefore, the Riemann curvature $R$ of the spacetime, as well as the flux $F$, is parallel with respect to the connection $\check{\nabla}$. These data are compatible with a Lorentzian homogeneous structure on the spacetime with canonical connection $\check{\nabla}$, which has torsion $(-2 / \sqrt{3}) H$ and curvature $(-2 / \sqrt{3}) F$, see Appendix C.1. Therefore, we shall take that the spacetime locally admits a Lorentzian transitive 6-dimensional group with Lie algebra $\mathfrak{g}=\mathfrak{h} \oplus \mathfrak{m}$ which has self-dual structure constants. The commutation relations are

$$
\left[m_{A}, m_{B}\right]=\frac{2}{\sqrt{3}}\left(F_{A B} h+H_{A B}{ }^{c} m_{C}\right), \quad\left[h, m_{A}\right]=\frac{2}{\sqrt{3}} F_{A}{ }^{B} m_{B},
$$

where $h$ is the generator of an abelian subalgebra $\mathfrak{h}$ and $\left\{m_{A}\right\}$ is a basis in $\mathfrak{m}$. Note that the indices have been raised with respect to the spacetime metric.

There are three Lorentzian 6-dimensional Lie algebras with self-dual structure constants which are isomorphic to

$$
\mathfrak{s l}(2, \mathbb{R}) \oplus \mathfrak{s u}(2), \quad \mathfrak{c w}_{6}, \quad \mathbb{R}^{5,1},
$$

where $\mathfrak{c m}_{6}$ is the Lie algebra of the $C W_{6}$ group manifold described in Appendix C.2. As the structure constants of $\mathbb{R}^{5,1}$ are zero, all the maximally supersymmetric solutions associated to it are locally isometric to the Minkowski space $\mathbb{R}^{4,1}$ with $F=0$.

Consider the commutation relations

$$
\left[t_{2}, t_{ \pm}\right]= \pm 2 t_{ \pm}, \quad\left[t_{+}, t_{-}\right]=t_{2} \text { and }\left[w_{a}, w_{b}\right]=2 \epsilon_{a b}^{c} w_{c},
$$

of $\mathfrak{s}(2, \mathbb{R})$ and $\mathfrak{s u}(2)$ Lie algebras, respectively, where $a, b, c=3,4$, 5. The most general choice of the generator $h$ which gives rise to a reductive Lorentzian 5-dimensional homogeneous space, see Appendix C.1, is $h=\alpha t_{2}+\beta w_{5}$, where $\alpha, \beta \in \mathbb{R}$. Then $\mathfrak{m}$ is spanned by $\left\{t_{ \pm}, p, u_{3}, u_{4}\right\}$, where $p=\alpha t_{2}-\beta u_{3}$. Let $\ell=\ell^{+} t_{+}+\ell^{-} t_{-}+\ell^{2} p+\ell^{3} u_{3}+\ell^{4} u_{4}$ be the left-invariant coframe on the homogeneous space. One can verify that for $\alpha, \beta \neq 0$ this homogeneous space admits a three parameter family of Lorentzian invariant metrics and a two-parameter family of invariant 2 -forms. However, imposing the relation between the structure constants as indicated in (165), and that $H$ is dual to $F$, one finds that the fields are

$$
\begin{aligned}
d s^{2} & =\frac{1}{\alpha^{2}} \ell^{+} \ell^{-}+\frac{1}{2 \beta^{2}}\left(\left(\ell^{3}\right)^{2}+\left(\ell^{4}\right)^{2}\right)+\frac{4 \alpha^{6}}{\beta^{6}}\left(\ell^{2}\right)^{2}, \\
F & =\frac{\sqrt{3}}{4 \alpha} \ell^{+} \wedge \ell^{-}+\frac{\sqrt{3}}{4 \beta} \ell^{3} \wedge \ell^{4} .
\end{aligned}
$$

The homogeneous space is locally isometric to $(S L(2, \mathbb{R}) \times S U(2)) / \mathbb{R}_{\alpha, \beta}$ where the inclusion of $\mathbb{R}$ in $S L(2, \mathbb{R}) \times S U(2)$ is $\left(\operatorname{diag}\left(e^{\alpha t}, e^{-\alpha t}\right), \operatorname{diag}\left(e^{i \beta t}, e^{-i \beta t}\right)\right.$ ). If either $\alpha=0$ or $\beta=0$, then the spacetime is locally isometric to $A d S_{3} \times S^{2}$ or $A d S_{2} \times S^{3}$ with magnetic or electric flux, respectively. These are the near horizon geometries of the magnetic string and extreme Reissner-Nordström black hole, respectively. While for $\alpha \beta \neq 0$, the maximally supersymmetric background is the near horizon geometry of the BMPV black hole.

The non-vanishing commutation relations of $\mathfrak{c w}_{6}$ are given in (C.11). As $\beta$ is skew-symmetric and non-degenerate it can be brought, up to an $O(4)$ transformation, to a block-diagonal form. Therefore, it can be determined by up to two real constants $\lambda_{1}$ and $\lambda_{2}$. Requiring that the structure constants are self-dual, one finds that $\lambda:=\lambda_{1}=\lambda_{2}$ and thus $\beta=\lambda d x^{1} \wedge d x^{2}+\lambda d x^{3} \wedge d x^{4}$. Two choices of a subalgebra of a Lie algebra related by a conjugation are considered as equivalent. Therefore the generator $h=h^{i} t_{i}+h^{-} t_{-}+h^{+} t_{+}$of the abelian subalgebra $\mathfrak{h}$ in $\mathfrak{c w}_{6}$ can be chosen up to a conjugation. For this observe that the adjoint action with $w=w^{i} t_{i}+w^{-} t_{-}+w^{+} t_{+}$gives

$$
h^{i} \rightarrow h^{i}-w^{-} \beta_{j}^{i} h^{j}+h^{-} \beta_{j}^{i} w^{j}, h^{-} \rightarrow h^{-}, h^{+} \rightarrow h^{+}-\beta_{i j} w^{i} h^{j} .
$$

Therefore all $h=h^{+} t_{+}$elements represent independent conjugacy classes in $\mathfrak{c w}_{6}$. On the other hand, if $h=h^{i} t_{i}+h^{+} t_{+}$, then the adjoint action acts on $h$ with translations on $h^{+}$and rotations on $h^{i}$ generated by $\beta$. Thus the independent conjugacy classes can be represented by $h=h^{i} t_{i}$ up to identifications of $h^{i}$ with rotations generated by $\beta$. Finally, if $h^{-} \neq 0$, then the adjoint action of $\mathfrak{c w}_{6}$ acts with translations on both $h^{i}$ and $h^{+}$. The former can be used to set $h^{i}=0$. In such case, the $h^{+}$remains invariant as the translations acting on it are $h^{i}$ dependent. The conjugacy classes can be represented by $h=h^{-} t_{-}+h^{+} t_{+}$. Thus for what follows it suffices to choose the generator of $\mathfrak{h}$ as either $h=h^{i} t_{i}$ or $h=h^{-} t_{-}+h^{+} t_{+}$.

First consider $h=h^{i} t_{i}$. Then without loss of generality one can choose $h=t_{4}$. This is because the $\mathfrak{c w}_{6}$ algebra has an underlying $S U(2) \times S O(2)$ symmetry generated by the anti-self-dual rotations acting on the generators $t_{i}$ which leave $\beta$ invariant. The $S O(2)$ symmetry is generated by $\beta$. These $S U(2)$ transformations can be used to set $h$ to the form above. If $\mathfrak{h}$ is spanned by $t_{4}$, then a basis in $\mathfrak{m}$ is $\left\{t_{-}, t_{+}, t_{1}, t_{2}, t_{3}\right\}$. A local section $s$ of the coset can be chosen by setting $x^{3}=x^{4}$, see Appendix C.1. A left-invariant coframe on the coset space, see (C.13), is 


$$
\begin{aligned}
& \ell^{-}=d v, \quad \ell^{a}=d x^{a}+\beta^{a}{ }_{b} x^{b} d v, \quad \ell^{3}=d x^{3}+\lambda x^{3} d v, \\
& \ell^{+}=d u+\frac{1}{2} \beta_{a b} x^{a} d x^{b}-\frac{1}{2} \lambda^{2} \delta_{a b} x^{a} x^{b} d v-\lambda^{2}\left(x^{3}\right)^{2},
\end{aligned}
$$

and the canonical connection is $\Psi=d x^{3}-\beta x^{3} d v$, where $a, b=1,2$. There are several invariant metrics that can be put on the coset space. However requiring that $H$ is skew-symmetric, one find that the most general one up to an overall scale is

$$
d s^{2}=2 \ell^{-} \ell^{+}+\delta_{a b} \ell^{a} \ell^{b}+\left(\ell^{3}\right)^{2} .
$$

After a change of coordinates $y^{a}=\left(e^{\frac{1}{2} v \beta}\right)^{a}{ }_{b} x^{b}, y^{3}=x^{3}$ and $u^{\prime}=u+\frac{1}{2} \lambda\left(x^{3}\right)^{2}$, one finds that

$$
\begin{aligned}
d s^{2} & =2 d v\left(d u-\frac{1}{2} \lambda^{2}\left(y^{3}\right)^{2} d v-\frac{1}{8} \lambda^{2} \delta_{a b} y^{a} y^{b} d v\right)+\delta_{a b} d y^{a} d y^{b}+\left(d y^{3}\right)^{3}, \\
F & =-\frac{\sqrt{3}}{2} \lambda d v \wedge d y^{3},
\end{aligned}
$$

where we have reset $u=u^{\prime}$ and $F$ can be read off after comparing the commutation relations of the homogeneous space $\mathfrak{h} \oplus \mathfrak{m}$ with those in (165). All the field equations and KSEs are satisfied. This is the maximally supersymmetric plane wave solution of $d=5$ minimal supergravity.

Next consider the conjugacy classes represented by $h=h^{-} t_{-}+h^{+} t_{+}$. If $\mathfrak{h}$ is spanned by $h=h^{-} t_{-}+h^{+} t_{+}$, then a basis in $\mathfrak{m}$ is $\left\{w, t_{i}\right\}$, where $w=h^{-} t_{-}-h^{+} t_{+}$. The non-vanishing commutation relations (C.11) in the basis adapted to the homogeneous space are

$$
\begin{aligned}
& {\left[h, t_{i}\right]=h^{-} \beta_{i}^{j} t_{j}, \quad\left[w, t_{i}\right]=\alpha \beta^{j} t_{j},} \\
& {\left[t_{i}, t_{j}\right]=-\frac{1}{2 h^{+}} \beta_{i j} h+\frac{1}{2 h^{+}} w .}
\end{aligned}
$$

Consider the local left-invariant coframe $\ell=\ell^{0} w+\ell^{i} t_{i}$, where a local section $s$ of the coset space has been chosen by setting $v=0$. The most general left-invariant metric on the homogeneous space is

$$
d s^{2}=\alpha\left(\ell^{0}\right)^{2}+\gamma_{i j} \ell^{i} \ell^{j},
$$

where $\alpha \in \mathbb{R}-\{0\}$ is a constant and $\gamma$ is a constant non-degenerate symmetric matrix. Comparing (173) with (165) and requiring that $H$ is skew-symmetric, one finds that $\gamma_{i j}=\delta_{i j}$ and $\alpha=-2 h^{-} h^{+}$. A similar comparison of the terms containing $F$ gives $h^{-} h^{+}=\frac{1}{2}$. Therefore the solution is

$$
d s^{2}=-\left(d u+\beta_{i j} x^{i} d x^{j}\right)^{2}+\delta_{i j} d x^{i} d x^{j}, \quad F=-\frac{\sqrt{3}}{2 \sqrt{2}} \beta_{i j} d x^{i} \wedge d x^{j},
$$

where we used the coordinates obtained in (C.13) and restricted the $C W_{6}$ left-invariant coframe to $v=0$. Furthermore, we have set $h^{+}=1$. This is the maximally supersymmetric Gödel universe solution of $d=5$ supergravity.

\subsection{Solutions of other $d=5$ supergravity KSEs and applications}

Following the work of [8] on the minimal ungauged $\mathcal{N}=1 d=5$ supergravity, numerous extensions of the programme have been made to other $d=5$ supergravities. First of these has been the classification of the supersymmetric solutions of minimal gauged supergravity [69] using the bilinears method. This has been used in [70] to find the first example of a supersymmetric asymptotically $A d S_{5}$ black hole with a regular event horizon. $A d S_{5}$ is the unique maximally supersymmetric solution of this theory. In the near-horizon limit, the black hole preserves 4 supersymmetries, however in the bulk it preserves only 2 supersymmetries. Further examples of supersymmetric black holes in minimal gauged supergravity have also been constructed [71,72]. A key difference between the gauged and ungauged theories in five dimensions is that the number of supersymmetries preserved in the gauged theory can be 2, 4, 6, or 8 while, as we have seen, in the ungauged theory they are either 4 or 8 . All solutions with 6 supersymmetries are locally isometric to $A d S_{5}$ [73], however it is known that there exist discrete quotients of $A d S_{5}$ which admit exactly 6 globally well-defined Killing spinors [62].

In terms of the description of the geometry, the $N=2$ solutions in the minimal gauged theory once again split into a timelike and a null class. As a consequence of the gravitino KSE, the spacetime in the timelike class, which is of particular relevance for black holes, is a local fibration over a 4-dimensional Kähler base space. The weakening of the geometric condition on the base space, when compared to the hyper-Kähler condition which arises in the ungauged theory, is consistent with the reduction in the amount of supersymmetry preserved. For the ungauged theory, solutions such as the BMPV black hole, and also the supersymmetric black ring found in [74], have base space $\mathbb{R}^{4}$. However, more general black hole geometries, such as those found in [75], as well as the black lens solutions of [76] and [77], are known to exhibit a Gibbons-Hawking base space. The Gibbons-Hawking metric is particularly useful as it allows for a complete integration of all of the supersymmetry conditions and field equations in terms of harmonic functions on $\mathbb{R}^{3}$, provided that the tri-holomorphic isometry extends to a symmetry of the full theory. There are no known examples of black hole solutions in a closed form for which the base space does not possess a tri-holomorphic isometry, though solutions of this type have been considered numerically [78]. In 
contrast, the Kähler base space for black holes in the gauged theory cannot be chosen to be hyper-Kähler. This is because the Ricci scalar of the base space cannot vanish as it must diverge at the location of the Killing horizon. Although the base space is singular, the 5-dimensional geometry remains regular at the horizon.

In addition, the solution of the KSEs of the ungauged $\mathcal{N}=1$ theory coupled to vector multiplets [79] has been used to find a large class of supersymmetric "black Saturn" solutions. These consist of a black hole with $S^{3}$ spherical horizon topology surrounded by arbitrarily many concentric black rings. The analogous result for the gauged $\mathcal{N}=1$ theory coupled to vector multiplets, [80] and [81], has been utilized to generalize further the black hole solution of [70]. Spinorial geometry techniques were also employed to classify all $N=4$ solutions in gauged supergravity coupled to vector multiplets in [82] and [83].

Black hole and black ring $[74,84]$ type solutions in the theories coupled to vector multiplets also lie within the timelike class of solutions. The presence of the vector multiplets does not affect the restriction on geometry on the 4-dimensional base space of the spacetime as derived for the minimal theories. In the ungauged theory and for a Gibbons-Hawking base space, the full solution can again be constructed explicitly. All explicitly known examples of black holes, rings and black Saturns have a Gibbons-Hawking base space. Large classes of candidate black hole microstate geometries, which are smooth and horizonless, have also been constructed using these techniques [85-89]. Closely related methods have also been used to construct examples of black rings with varying charge density [90], though it has been shown that such solutions typically do not possess smooth horizons [91]. One notable property of the Gibbons-Hawking manifolds that has been utilized to describe microstate geometries is that they are ambipolar, i.e. the signature flips from +4 to -4 across certain surfaces, though the five-dimensional solution retains a standard signature, and is regular. The geometry of such ambipolar base spaces has been considered in [92].

More recent work has been done to find the solutions of the KSEs in generic $d=5$ supergravities. In [93], all such solutions of the ungauged theory coupled to vector multiplets and hypermultiplets have been presented. In the timelike case, the presence of the hypermultiplets implies that the 4-dimensional base space used to describe such solutions is no longer hyper-Kähler, but rather admits a weaker set of conditions. Further extension of this work has been made in [94], where all solutions of the KSEs of the (non-abelian) gauged theory coupled to arbitrary many vector, tensor and hypermultiplets have been described, and new examples of solutions have been found. Further work on the solution of the KSEs of $\mathcal{N}=4$ supergravity has been done in [95].

Higher derivative supergravity solutions have also been investigated in some detail. In [96-98], an off-shell formalism together with the bilinears method has been employed to consider solutions of the theory comprising of the Weyl multiplet, coupled to arbitrary many (ungauged) vector multiplets, and one hypermultiplet. Spinorial geometry techniques have also been used to classify the solutions [99]. This theory lies within a large class of higher-derivative supergravity theories constructed in [100-102]. In this case, the structure of the gravitino KSE is identical to that given in (97), but with $F$ replaced by an auxiliary 2-form field which lies within the Weyl multiplet. As a consequence, some of the geometric conditions are common to those of the minimal 2-derivative theory; for example in the timelike case the base space is again hyperKähler. However, the remaining conditions on the geometry are modified in a highly nontrivial fashion due to the curvature couplings. Other types of higher-derivative 5-dimensional supergravities are known to exist, such as [103] and [104], and it would be of interest to further extend the classification programme to these theories.

\section{Minimal $\mathcal{N}=(1,0) d=6$ supergravity}

Theories in 6 dimensions have played a significant role in the investigation of string dualities and brane dynamics, see e.g. $[2,105,106]$. For example, it has been argued that IIA string theory on $\mathbb{R}^{5,1} \times K_{3}$ is dual to the heterotic string theory on $\mathbb{R}^{5,1} \times T^{4}$. There is also evidence for a $d=6$ self-dual string theory [107]. Moreover, it is expected from the AdS/CFT correspondence that a $d=6$ superconformal theory models the dynamics of coincident multiple M5-branes.

Here we shall focus on the solution of the KSE of minimal $\mathcal{N}=(1,0) d=6$ supergravity theory [108], i.e. 8 real supercharges. This solution has been worked out by the authors of [109] using the bilinears method. The maximal supersymmetric solutions have also been found in [110]. The solution of the KSEs of the $\mathcal{N}=(1,0)$ gauged supergravity theory coupled to any number of tensor, vector and scalar multiplets [111-113] have been given by the authors of [114] using the spinorial geometry method.

\subsection{Fields and solution of the KSE}

\subsubsection{Fields and KSE}

The bosonic fields of minimal $\mathcal{N}=(1,0) d=6$ supergravity are a metric and an anti-self-dual 3-form field strength $H$ which is closed, $d H=0$. The KSE of the theory is the vanishing condition of the gravitino supersymmetry variation evaluated at the locus where the gravitino vanishes. This reads as

$$
\hat{\nabla}_{A} \epsilon=0,
$$

where $\hat{\nabla}$ is a connection with skew-symmetric torsion $H$

$$
\hat{\nabla}_{A} Y^{B}:=\nabla_{A} Y^{B}+\frac{1}{2} H^{B}{ }_{A C} Y^{C},
$$


and $\epsilon$ is a symplectic Majorana-Weyl spinor. Clearly (176) is a parallel transport equation with respect to a connection with holonomy contained in $\operatorname{Spin}(5,1)$. A similar gravitino KSE also arises in heterotic supergravity which will be investigated in Section 7. The symplectic-Majorana condition on $\epsilon$ will be explained later in the description of the spinors of the theory.

The integrability condition of the gravitino KSE (176) is

$$
\hat{R}_{A B, C D} \Gamma^{C D} \epsilon=0,
$$

where $\hat{R}$ is the curvature of $\hat{\nabla}$. Using that $d H=0$ and that $H$ is anti-self-dual, one also finds that

$$
\Gamma^{B} \hat{R}_{A B, C D} \Gamma^{C D} \epsilon=-2 E_{A B} \Gamma^{B} \epsilon=0,
$$

where $E_{A B}=0$ is the Einstein field equation of the theory.

\subsubsection{Spinors}

Given a pair of Weyl $\operatorname{Spin}(5,1)$ spinors $\epsilon^{a}$, the symplectic-Majorana reality condition is $\epsilon^{a}=\epsilon^{a b} C * \epsilon_{b}^{T}$, where $C$ is the charge conjugation matrix and $\epsilon^{a b}$ is the symplectic invariant form of $S p(1)$. It arises for supersymmetric theories in $d=6$ because $\operatorname{Spin}(5,1)$ does not admit a real spin representation which is needed for the supersymmetry transformations to preserve the reality of the bosonic fields of the theory. Clearly $\epsilon^{1}$ and $\epsilon^{2}$ are not linearly independent.

The most effective way to understand the symplectic-Majorana $\operatorname{Spin}(5,1)$ spinors is to identify them with the $S U(2)$ invariant Majorana-Weyl spinors of $\operatorname{Spin}(9,1)[114,115]$. To do this explicitly, the Dirac spinors of $\operatorname{Spin}(5,1)$ and $\operatorname{Spin}(9,1)$ are identified with $\Lambda^{*}\left(\mathbb{C}^{3}\right)$ and $\Lambda^{*}\left(\mathbb{C}^{5}\right)$, respectively. Positive chirality spinors are the even degree forms while the negative chirality spinors are the odd degree forms. A realization of the gamma matrices of both Clifford algebras $\operatorname{Cliff}\left(\mathbb{R}^{5,1}\right)$ and Cliff $\left(\mathbb{R}^{9,1}\right)$ is given in Appendix B.

Given a Hermitian basis $\left\{e_{1}, \ldots, e_{5}\right\}$ in $\mathbb{C}^{5}, \operatorname{Cliff}\left(\mathbb{R}^{5,1}\right)$ can be included in $\operatorname{Cliff}\left(\mathbb{R}^{9,1}\right)$ by choosing the subspace $\mathbb{C}^{3}$ in $\mathbb{C}^{5}$ as $\mathbb{C}^{3}=\mathbb{C}\left\langle e_{1}, e_{2}, e_{5}\right\rangle$. Therefore the positive chirality Weyl spinors of $\operatorname{Spin}(5,1)=S L(2, \mathbb{H})$ are given by $\Lambda^{\mathrm{ev}}\left(\mathbb{C}\left\langle e_{1}, e_{2}, e_{5}\right\rangle\right)=\mathbb{H}^{2}$. The symplectic Majorana-Weyl condition of $\operatorname{Spin}(5,1)$ is the Majorana-Weyl condition of $\operatorname{Spin}(9,1)$ spinors, i.e.

$$
\epsilon^{*}=\Gamma_{6789} \epsilon,
$$

where $\epsilon \in \Lambda^{\mathrm{ev}} \mathbb{C}\left\langle e_{1}, e_{2}, e_{5}\right\rangle \otimes \Lambda^{*} \mathbb{C}\left\langle e_{34}\right\rangle$. In particular, a basis for the symplectic Majorana-Weyl spinors is

$$
\begin{array}{ll}
1+e_{1234}, & i\left(1-e_{1234}\right), \quad e_{12}-e_{34}, \quad i\left(e_{12}+e_{34}\right), \\
e_{15}+e_{2534}, \quad i\left(e_{15}-e_{2534}\right), \quad e_{25}-e_{1534}, \quad i\left(e_{25}+e_{1534}\right) .
\end{array}
$$

Observe that the above basis selects the diagonal of two copies of the Weyl representation of $\operatorname{Spin}(5,1)$, where the first copy is $\Lambda^{\mathrm{ev}}\left(\mathbb{C}\left\langle e_{1}, e_{2}, e_{5}\right\rangle\right)$ while the second copy is $\Lambda^{\mathrm{ev}}\left(\mathbb{C}\left\langle e_{1}, e_{2}, e_{5}\right\rangle\right) \otimes \mathbb{C}\left\langle e_{34}\right\rangle$. The $S U(2)=S p(1)$ group, whose Lie algebra generators are

$$
\rho^{1}=\frac{1}{2}\left(\Gamma_{38}+\Gamma_{49}\right), \quad \rho^{2}=\frac{1}{2}\left(\Gamma_{89}-\Gamma_{34}\right), \quad \rho^{3}=\frac{1}{2}\left(\Gamma_{39}-\Gamma_{48}\right),
$$

acts on the auxiliary directions $e_{3}$ and $e_{4}$ and leaves the basis (181) invariant.

\subsubsection{Solution of the gravitino KSE}

In the absence of matter multiplets, supersymmetric solutions of the minimal $\mathcal{N}=(1,0)$ theory preserve either $N=4$ or $N=8$ supersymmetries, i.e. the solutions are either half supersymmetric or maximally supersymmetric.

To see this, observe that the KSE (176) is covariant under the action of $\operatorname{Spin}(5,1)$. This can be used to choose a representative for the first Killing spinor. As $\operatorname{Spin}(5,1)=S L(2, \mathbb{H})$ and the space of spinors is essentially identified with $\mathbb{H}^{2}$, one can always choose the first Killing spinor as $\epsilon=1+e_{1234}$.

Next, it is straightforward to see that the covariant derivative $\hat{\nabla}$ in the gravitino KSE (176) commutes with the generators of $S p(1)$ in (182). So if $\epsilon=1+e_{1234}$ is a Killing spinor, then all of the first four spinors in (181)

$$
1+e_{1234}, i\left(1-e_{1234}\right), \quad e_{12}-e_{34}, \quad i\left(e_{12}+e_{34}\right),
$$

will also be Killing. Therefore the backgrounds must preserve four supersymmetries. The isotropy group of all four Killing spinors is $\operatorname{Sp}(1) \times \mathbb{H}$ in $\operatorname{Spin}(5,1)$. Therefore $\operatorname{hol}(\hat{\nabla}) \subseteq S p(1) \times \mathbb{H}$.

Furthermore, if a background admits an additional Killing spinor to the first four already chosen, then it will preserve all supersymmetries and will thus be maximally supersymmetric. This can be easily seen upon inspection of the basis (181) and after the application of the argument above with the generators of $S p(1)$ in (182).

\section{2. $N=4$ solutions}

\subsubsection{Geometry}

As the only KSE of minimal $\mathcal{N}=(1,0)$ supergravity theory is the gravitino KSE, the geometry of the background is characterized by the assertion that the holonomy of supercovariant connection $\hat{\nabla}$ is contained in the isotropy group of the 
Killing spinors in $\operatorname{Spin}(5,1)$, hol $(\hat{\nabla}) \subseteq \operatorname{Sp}(1) \ltimes \mathbb{H}$. To investigate the consequences of this on the geometry of spacetime, one can compute the form bi-linears. Given two spinors $\epsilon_{1}$ and $\epsilon_{2}$, these are given by

$$
\tau=\frac{1}{k !} B\left(\epsilon_{1}, \Gamma_{A_{1} \ldots A_{k}} \epsilon_{2}\right) \mathbf{e}^{A_{1}} \wedge \cdots \wedge \mathbf{e}^{A_{k}},
$$

where $B$ is the Majorana inner product of $\operatorname{Spin}(9,1)$, see Appendix $B$, and $\mathbf{e}^{A}$ is a spacetime coframe. Assuming that $\epsilon_{1}$ and $\epsilon_{2}$ satisfy the gravitino KSE, it is easy to see that

$$
\hat{\nabla}_{A} \tau=0 .
$$

The forms $\tau$ are covariantly constant with respect to $\hat{\nabla}$.

Applying this to the $N=4$ backgrounds under investigation, the linearly independent bi-linears of the Killing spinors (183) in the spinorial geometry coframe, see (B.12) in Appendix B, are

$$
X:=\mathbf{e}^{-}, \quad \tau_{r}:=\mathbf{e}^{-} \wedge \omega_{r}, \quad r=1,2,3,
$$

where

$$
\begin{aligned}
& \omega_{1}=-i \delta_{\alpha \bar{\beta}} \mathbf{e}^{\alpha} \wedge \mathbf{e}^{\bar{\beta}}, \quad \omega_{2}=-\mathbf{e}^{1} \wedge \mathbf{e}^{2}-\mathbf{e}^{\overline{1}} \wedge \mathbf{e}^{\overline{2}}, \\
& \omega_{3}=i\left(\mathbf{e}^{1} \wedge \mathbf{e}^{2}-\mathbf{e}^{\overline{1}} \wedge \mathbf{e}^{\overline{2}}\right), \quad \alpha, \beta=1,2 .
\end{aligned}
$$

Therefore $X$ is a null one-form and $\omega_{r}$ are the fundamental forms of $S p(1)$. Note that in the same coframe the metric is written as $d s^{2}=2 \mathbf{e}^{-} \mathbf{e}^{+}+2 \delta_{\alpha \bar{\beta}} \mathbf{e}^{\alpha} \mathbf{e}^{\bar{\beta}}$.

In what follows it is convenient to choose the real coframe basis $\left\{\mathbf{e}^{i}\right\}=\left\{\mathbf{e}^{\alpha}, \mathbf{e}^{\bar{\alpha}}\right\}$ and write the metric and 3-form $H$ as

$$
\begin{aligned}
d s^{2}= & 2 \mathbf{e}^{-} \mathbf{e}^{+}+\delta_{i j} \mathbf{e}^{i} \mathbf{e}^{j}, \\
H= & H_{-+i} \mathbf{e}^{-} \wedge \mathbf{e}^{+} \wedge \mathbf{e}^{i}+\frac{1}{2} H_{+i j} \mathbf{e}^{+} \wedge \mathbf{e}^{i} \wedge \mathbf{e}^{j} \\
& +\frac{1}{2} H_{-i j} \mathbf{e}^{-} \wedge \mathbf{e}^{i} \wedge \mathbf{e}^{j}+\tilde{H},
\end{aligned}
$$

where $\tilde{H}=\frac{1}{3 !} H_{i j k} \mathbf{e}^{i} \wedge \mathbf{e}^{j} \wedge \mathbf{e}^{k}$. The anti-self duality of $H$ implies that $H_{+i j}$ is anti-self-dual and $H_{-i j}$ is self-dual as 2 -forms in the directions transverse to the lightcone, and $H_{i j k}=\epsilon_{i j k}^{l} H_{-+l}$.

The choice of the local coframe $\left\{\mathbf{e}^{-}, \mathbf{e}^{+}, \mathbf{e}^{i}: i=1, \ldots, 4\right\}$ is not unique. The isotropy group of the Killing spinors, $S p(1) \ltimes \mathbb{R}^{8}$, acts on the coframe with local transformations as

$$
\mathbf{e}^{-} \rightarrow \mathbf{e}^{-}, \quad \mathbf{e}^{+} \rightarrow \mathbf{e}^{+}-q_{i} O_{j}^{i} \mathbf{e}^{j}-\frac{1}{2} q^{2} \mathbf{e}^{-}, \quad \mathbf{e}^{i} \rightarrow O_{j}^{i} \mathbf{e}^{j}+q^{i} \mathbf{e}^{-},
$$

where $O$ takes values in $S p(1)$ and $q$ in $\mathbb{H}$. So there is no natural way to choose the transverse directions to the lightcone. This is a special case of a more general phenomenon that occurs in all supersymmetric backgrounds that admit Killing spinors which have isotropy group $K \ltimes \mathbb{R}^{m}$ in a spin group, where $K$ is a compact group, see also Section 7. The associated coframe transforms as in (189) with $O \in K$ and $q \in \mathbb{R}^{m}$.

Nevertheless one can proceed as follows. The existence of a parallel null 1 -form $\mathbf{e}^{-}$defines a trivial sub-bundle $I$ in the cotangent bundle $T^{*} M$ of spacetime. Consider the orthogonal sub-bundle of $I, I^{\perp}$, in $T^{*} M$. As the fibres of $I$ are along the null direction $\mathbf{e}^{-}$, the fibres of $I^{\perp}$ are spanned by $\left\{\mathbf{e}^{-}, \mathbf{e}^{i}: i=1, \ldots, 4\right\}$ so $I \subset I^{\perp}$. The "transverse bundle" (or screening space) to the lightcone, $\mathcal{T}$, is defined as $\mathcal{T}=I^{\perp} / I$. This description of $\mathcal{T}$ generalizes to all backgrounds with Killing spinors that have isotropy group $K \ltimes \mathbb{R}^{m}$.

The 2-forms $\omega_{r}$ in (187) are sections of $\Lambda^{2}(\mathcal{T})$, where we have identified $\mathcal{T}$ and its dual with the transverse metric $\tilde{g}=\delta_{i j} \mathbf{e}^{i} \mathbf{e}^{j}$. Furthermore one can define (almost) complex structures $I_{r}$ on $\mathcal{T}, \omega_{r}(\tilde{Y}, \tilde{Z})=\tilde{g}\left(\tilde{Y}, I_{r} \tilde{Z}\right)$, where $\tilde{Y}=\tilde{Y}{ }^{i} \mathbf{e}_{i}$ and similarly for $\tilde{Z}$. One can verify that $I_{1}^{2}=I_{2}^{2}=-\mathbf{1}, I_{1} I_{2}=-I_{2} I_{1}$ and $I_{3}=I_{1} I_{2}$.

The conditions that the gravitino KSE imposes on the spacetime geometry can be written as

$$
\hat{\nabla}_{A} X=0, \quad \hat{\nabla}_{A} \tau_{r}=0 .
$$

The first condition in (190) implies that

$$
i_{X} H=d X, \quad \mathcal{L}_{X} g=0 .
$$

Therefore the $i_{X} H$ component of $H$ is determined in terms of the geometry and $X$ is a Killing vector field on the spacetime $M$. As $H$ is closed, the first condition in (191) also implies that $H$ is invariant under the action of the vector field $X, \mathcal{L}_{X} H=0$.

It remains to solve the second condition in (190). This can be decomposed as

$$
\begin{aligned}
\nabla_{+}\left(\omega_{r}\right)_{i j} & =H_{+}{ }^{k}{ }_{[i}\left(\omega_{r}\right)_{j] k}=0, \\
\nabla_{-}\left(\omega_{r}\right)_{i j} & =H_{-}{ }^{k}{ }_{[i}\left(\omega_{r}\right)_{j] k}, \\
\hat{\nabla}_{i}\left(\omega_{r}\right)_{j k} & =\nabla_{i}\left(\omega_{r}\right)_{j k}+H^{m}{ }_{i j j}\left(\omega_{r}\right)_{k] m}=0,
\end{aligned}
$$


where we have used that in the coframe $\left\{\mathbf{e}^{-}, \mathbf{e}^{+}, \mathbf{e}^{i}: i=1, \ldots, 4\right\}$ the condition $\operatorname{hol}(\hat{\nabla}) \subseteq S p(1) \times \mathbb{H}$ implies that $\hat{\Omega}_{A,+B}=0$. The right-hand-side of the first condition in (192) vanishes because $H_{+i j}$ is anti-self-dual while $\omega_{r}$ are self-dual forms. Thus $\nabla_{X} \omega_{r}=0$ and as a result

$$
\mathcal{L}_{X}\left(\mathbf{e}^{-} \wedge \omega_{r}\right)=0 .
$$

On the other hand the second condition in (192), together with the fact that $H_{-i j}$ is self-dual, implies that $H_{-i j}$ is entirely determined in terms of the geometry. The last condition in (192) is that which one expects for a manifold with metric $d \tilde{s}^{2}=\delta_{i j} \mathbf{e}^{i} \mathbf{e}^{j}$, torsion $\tilde{H}$ and cotangent bundle $\mathcal{T}$ to admit a HKT structure [116]. As $\mathcal{T}$ has rank four, the HKT condition implies that the complex structures $I_{r}$ are integrable. After solving all the conditions that arise from the KSE, the fields can be written as

$$
\begin{gathered}
d s^{2}=2 \mathbf{e}^{-} \mathbf{e}^{+}+\delta_{i j} \mathbf{e}^{i} \mathbf{e}^{j}, \\
H=\mathbf{e}^{+} \wedge d \mathbf{e}^{-}-\left(\frac{1}{16}\left(\omega_{r}\right)_{k l} \nabla_{-}\left(\omega_{s}\right)^{k l} \epsilon^{r s t}\right)\left(\omega_{t}\right)_{i j} \mathbf{e}^{-} \wedge \mathbf{e}^{i} \wedge \mathbf{e}^{j} \\
\quad-\frac{1}{3 !}\left(d \mathbf{e}^{-}\right)_{-\ell} \epsilon_{i j k}^{\ell} \mathbf{e}^{i} \wedge \mathbf{e}^{j} \wedge \mathbf{e}^{k} .
\end{gathered}
$$

It is clear that $H$ is determined in terms of the geometry.

Before we proceed with the investigation of the geometry of these backgrounds in more detail, and describe the explicit solutions, let us comment on the solution of the KSEs of gauged $(1,0)$ supergravity coupled to any number of tensor, vector and scalar multiplets which has been carried out in [114]. First, the holonomy of the supercovariant connection of such a theory is in $\operatorname{Spin}(5,1) \cdot \operatorname{Sp}(1)$ instead of $\operatorname{Spin}(5,1)$, where the additional $\operatorname{Sp}(1)$ subgroup is due to the inclusion of scalar multiplets. There are also a priori backgrounds that preserve $N=1, N=2, N=3, N=4$ and $N=8$ supersymmetries. Furthermore there are two kinds of bilinears. Those that are forms on the spacetime, like $\mathbf{e}^{-}$, and those that are forms which are twisted with an $S p(1)$ bundle like $\mathbf{e}^{-} \wedge \omega_{r}$. The additional $S p(1)$ twist changes the geometry of the spacetime. For example the geometry of the $N=1$ backgrounds is now based on quaternionic Kähler geometries with torsion [117] instead of HKT geometries. Of course there are many more additional conditions that arise from the KSEs of the matter multiplets. For more details, see the original publication.

\subsubsection{Special coordinates}

To give a local description of the geometry of $S p(1) \ltimes \mathbb{H}$ backgrounds, one can proceed as follows. First let us adapt a coordinate $u$ along the $\hat{\nabla}$ parallel vector field $X=\partial_{u}$. Then a coframe can be chosen as

$$
\begin{aligned}
& \mathbf{e}^{-}=W^{-1}\left(d v+m_{I} d y^{I}\right), \quad \mathbf{e}^{+}=d u+V d v+n_{I} d y^{I}, \\
& \mathbf{e}^{i}=\tilde{e}_{I}^{i} d y^{I}+p^{i} d v,
\end{aligned}
$$

where $v, y^{I}$ are the rest of the spacetime coordinates and $W, V, \tilde{e}$ and $p$ can depend on all spacetime coordinates. After performing a coframe rotation as in (189) with $O=\mathbf{1}$, one can set $p=0$ after a possible redefinition of $V, n$ and $\tilde{e}$. Note that this is equivalent to choosing a splitting $I^{\perp}=I \oplus \mathcal{T}$.

As all the geometric data of the theory, including the metric, $H$ and the fundamental forms in (186), are invariant under $X$, a coframe can also be chosen such that it is independent of $u$. In such a case, the expression for the 3-form flux in (194) can be simplified to

$$
H=d\left(\mathbf{e}^{-} \wedge \mathbf{e}^{+}\right)-\frac{1}{3 !}\left(d \mathbf{e}^{-}\right)_{-\ell} \epsilon_{i j k}^{\ell} \mathbf{e}^{i} \wedge \mathbf{e}^{j} \wedge \mathbf{e}^{k},
$$

where $\mathbf{e}^{i}=\tilde{e}_{l}^{i} d y^{l}$. As a result $d H=0$ leads to a harmonic-like condition on $W$ which will be explored to find solutions.

\subsubsection{Solutions}

Before we proceed to give some examples of solutions, the KSE implies that some of the field equations of the theory are automatically satisfied. To see this observe that the integrability condition (179) upon taking an appropriate Majorana inner product with $\epsilon$ implies that $E_{A-}=0$. Using this and acting on (179) with $E_{A C} \Gamma^{C}$, one finds that $E_{A i} E_{A}{ }^{i}=0$ as $\epsilon \neq 0$, where there is no summation over $A$. Thus all field equations are implied provided that one imposes $E_{--}=0$ and the Bianchi identity $d H=0$.

A large class of solutions can be found after assuming in addition that $\partial_{v}$ leaves all the fields invariant. Then the spacetime can be viewed locally as a fibration having fibre $\mathbb{R}^{2}$ with coordinates $(u, v)$ over a base space $B^{4}$ which is a 4-dimensional HKT manifold. The co-tangent space $T^{*} B^{4}$ of the HKT manifold is identified with $\mathcal{T}$. It follows from (195) and (196) that $\tilde{H} \sim \star_{4}\left(W^{-1} d W\right)$. It is known that 4-dimensional HKT manifolds with a co-exact torsion $\tilde{H}$ are conformal to hyper-Kähler. Collecting these data together, the metric and 3-form field strength can be written as

$$
d s^{2}=2 \mathbf{e}^{-} \mathbf{e}^{+}+W d s_{\mathrm{hk}}^{2}, \quad H=d\left(\mathbf{e}^{-} \wedge \mathbf{e}^{+}\right)+\star_{\mathrm{hk}} d W,
$$

where $\left\{\mathbf{e}^{-}, \mathbf{e}^{+}\right\}$are given in (195) but now all components are independent of both the coordinates $u$ and $v, d s_{\mathrm{hk}}^{2}$ is a hyperKähler metric and the Hodge duality operation has been taken with respect to $d s_{\mathrm{hk}}^{2}$ as indicated. 
These backgrounds solve the KSEs and the field equations provided that

$$
\begin{gathered}
\nabla_{\mathrm{hk}}^{2} W=\nabla_{\mathrm{hk}}^{2} V=0, \quad \star_{\mathrm{hk}} d m=-d m, \\
\star_{\mathrm{hk}}(d n-W d V \wedge m)=(d n-W d V \wedge m),
\end{gathered}
$$

i.e. $W$ and $V$ are harmonic functions on the hyper-Kähler manifold $B^{4}$. The remaining two conditions in (198) are implied by the requirement that $H_{+i j}$ and $H_{-i j}$ are anti-self-dual and self-dual, respectively.

For $\mathbf{e}^{-} \wedge d \mathbf{e}^{-}=0$, i.e. $m=0$, the solutions have the interpretation of rotating dyonic strings with a pp-waves propagating on them. The space transverse to the strings is the hyper-Kähler manifold $B^{4}$. For $B^{4}=\mathbb{R}^{4}$, one obtains planar dyonic strings with $W=1+\left(Q_{s} /|y|^{2}\right)$ and $V=Q_{w} /|y|^{2}$, where $Q_{s}$ is the charge of the string and $Q_{w}$ is the momentum along the wave. For $V=0$, one recovers the dyonic string of [5]. A solution for $n$ can also be found, see e.g. [118]. Many more solutions can be constructed for different choices of the hyper-Kähler metric, such as those used to describe certain black hole microstate geometries $[119,120]$, which are described by a Gibbons-Hawking base space. An extensive investigation of the solutions can be found in [121-123].

\subsection{Maximally supersymmetric backgrounds}

For the maximally supersymmetric backgrounds $\hat{R}=0$. Therefore, these are parallelizable 6-dimensional Lorentzian manifolds with respect to a connection with skew-symmetric torsion. These are Lorentzian signature group manifolds equipped with the bi-invariant metric, and up to a local isometry they can be identified with

$$
\mathbb{R}^{5,1}, \quad S L(2, \mathbb{R}) \times S U(2), \quad C W_{6},
$$

where $H$ is given in terms of their structure constants. The anti-self-duality condition on $H$ implies that the radii of $\operatorname{SL}(2, \mathbb{R})$ and $S U(2)$ must be equal and that the structure constants $\beta$ of the Cahen-Wallach group manifold $C W_{6}$ are self-dual, see Appendix C.2.

\section{Geometry of heterotic supergravity backgrounds}

The effective theory of the heterotic string $[124,125]$ can be described by a type I theory, i.e. a theory with 16 supercharges in 10 dimensions, which includes higher curvature corrections. These can be organized in an expansion in terms of the string length square parameter, $\alpha^{\prime}$, and coupling constant, $g_{s}$. The $\alpha^{\prime}$ corrections can be computed by a sigma model loop calculation. The light bosonic fields of heterotic strings are the spacetime metric $g$, the NS-NS 3-form field strength $H$, the dilaton $\Phi$ and the 2 -form gauge field $F$ with gauge groups either $E_{8} \times E_{8}$ or $S O(32) / \mathbb{Z}_{2}$. These theories exhibit several attractive features as they are chiral and upon a compactification on 6-dimensional Calabi-Yau manifolds give rise to $\mathcal{N}=1$ theories in 4 dimensions. As a result they have been extensively explored in string phenomenology.

The feature that will be described here is the solution the KSEs of heterotic supergravity. This has been done in all cases $[115,126,127]$ and as a result the geometry of all backgrounds that preserve some of the supersymmetry of the underlying theory can be systematically described. Progress towards the solution of the KSEs of the related common sector in type II theories has also been made, see e.g. [128].

\subsection{Fields, KSEs, integrability conditions and spinors}

\subsubsection{KSEs and field equations}

The world-sheet theory of the heterotic string is chiral and it exhibits an anomaly which is cancelled by an anomaly cancellation mechanism [129]. This modifies the Bianchi identity of the three-form field strength $H$ as

$$
d H=-\frac{1}{4} \alpha^{\prime}\left(\operatorname{tr} \tilde{R}^{2}-\operatorname{tr} F^{2}\right)+\mathcal{O}\left(\alpha^{\prime 2}\right),
$$

where $\tilde{R}$ is the curvature of a spacetime connection $\tilde{\nabla}$. The choice of $\tilde{R}$ is renormalization scheme dependent and we shall leave $\tilde{R}$ arbitrary at the moment.

Up to and including two-loops in the sigma model perturbation theory [130], the KSEs of the effective theory [131-133] can be written as

$$
\begin{aligned}
\mathcal{D}_{M} \epsilon & :=\hat{\nabla}_{M} \epsilon+\mathcal{O}\left(\alpha^{\prime 2}\right)=0, \\
\mathcal{A} \epsilon & :=\left(\Gamma^{M} \partial_{M} \Phi-\frac{1}{12} H_{M N L} \Gamma^{M N L}\right) \epsilon+\mathcal{O}\left(\alpha^{\prime 2}\right)=0, \\
\mathcal{F} \epsilon & :=F_{M N} \Gamma^{M N} \epsilon+\mathcal{O}\left(\alpha^{\prime 2}\right)=0,
\end{aligned}
$$

where

$$
\hat{\nabla}_{N} Y^{M}:=\nabla_{N} Y^{M}+\frac{1}{2} H^{M}{ }_{N R} Y^{R} .
$$


The first equation is the gravitino KSE for a metric connection $\hat{\nabla}$ with torsion given by the 3 -form field strength $H$. The second equation is the dilatino KSE and the last is the gaugino KSE. The KSEs retain their one loop form as they do not receive an explicit 2-loop contribution.

Furthermore, the field equations of the theory after including the two-loop sigma model correction are

$$
\begin{aligned}
E_{M N}:= & R_{M N}+\frac{1}{4} H^{R}{ }_{M L} H^{L}{ }_{N R}+2 \nabla_{M} \partial_{N} \Phi \\
& +\frac{\alpha^{\prime}}{4}\left[\tilde{R}_{M P, Q R} \tilde{R}_{N}{ }^{P, Q R}-F_{M P a b} F_{N}{ }^{P a b}\right]+\mathcal{O}\left(\alpha^{\prime 2}\right)=0, \\
L H_{P R}:= & \nabla_{M}\left[e^{-2 \Phi} H^{M}{ }_{P R}\right]+\mathcal{O}\left(\alpha^{\prime 2}\right)=0, \\
L \Phi:= & \nabla^{2} \Phi-2 \nabla_{M} \Phi \nabla^{M} \Phi-\frac{1}{12} H_{M N P} H^{M N P} \\
& +\frac{\alpha^{\prime}}{12}\left(\tilde{R}_{N_{1} N_{2} N_{3} N_{4}} \tilde{R}^{N_{1} N_{2} N_{3} N_{4}}-F_{M N a b} F^{M N a b}\right)+\mathcal{O}\left(\alpha^{2}\right)=0, \\
L F_{M}:= & \hat{\nabla}^{M}\left[e^{-2 \Phi} F_{M N}\right]+\mathcal{O}\left(\alpha^{\prime 2}\right)=0 .
\end{aligned}
$$

The Einstein and dilaton field equations receive a 2-loop contribution while the field equations for $H$ and $F$ retain their one-loop form.

In the investigation of solutions of the theory two distinct cases have been considered distinguished by whether the anomaly contribution to $\mathrm{dH}$ and the two-loop contribution to the field equations vanish or not. These can vanish provided an appropriate choice is made for $F$ and $\tilde{R}$, i.e. $F=\tilde{R}$. Of course in such a case $d H=0$.

For the solution of the KSEs of heterotic supergravity that follows, we shall initially assume that both the anomaly and the two-loop contributions to the field equations vanish. Later in Section 7.5, we shall explain how our analysis can be modified to describe the geometry of supersymmetric solutions with non-vanishing anomaly and two-loop contributions.

\subsubsection{Integrability conditions of KSES}

It is well-known that the KSEs imply some of the field equations of supersymmetric backgrounds. To investigate this, as well as to identify some additional consistency conditions required for the compatibility between the Bianchi identity, the KSEs and the field equations of the theory, see Section 7.5, let us consider the integrability conditions of the KSEs (201) of the heterotic supergravity. These are, see also [134],

$$
\begin{array}{r}
\mathcal{R}_{M N} \epsilon:=\left[\mathcal{D}_{M}, \mathcal{D}_{N}\right] \epsilon=\frac{1}{4} \hat{R}_{M N, A B} \Gamma^{A B} \epsilon=\mathcal{O}\left(\alpha^{2}\right), \\
{\left[\mathcal{D}_{M}, \mathcal{F}\right] \epsilon=\left[\hat{\nabla}_{M}, F_{N L} \Gamma^{R S}\right] \epsilon=\mathcal{O}\left(\alpha^{\prime 2}\right),} \\
{\left[\mathcal{D}_{M}, \mathcal{A}\right] \epsilon=\left[\hat{\nabla}_{M}, \partial_{N} \Phi \Gamma^{N}-\frac{1}{12} H_{N P Q} \Gamma^{N P Q}\right] \epsilon=\mathcal{O}\left(\alpha^{\prime 2}\right),} \\
{[\mathcal{F}, \mathcal{A}] \epsilon=\left[F_{R S} \Gamma^{R S}, \partial_{N} \Phi \Gamma^{N}-\frac{1}{12} H_{N P Q} \Gamma^{N P Q}\right] \epsilon=\mathcal{O}\left(\alpha^{\prime 2}\right) .}
\end{array}
$$

Multiplying the first expression above with $\Gamma^{N}$ and using appropriately the remaining integrability conditions, one finds that

$$
\begin{aligned}
-2 E_{M N}^{(0)} \Gamma^{N} \epsilon-e^{2 \Phi} L H_{M N} \Gamma^{N} \epsilon-\frac{1}{6} B H_{M A B C} \Gamma^{A B C} \epsilon & =\mathcal{O}\left(\alpha^{\prime 2}\right), \\
L \Phi^{(0)} \epsilon-\frac{1}{4} e^{2 \Phi} L H_{M N} \Gamma^{M N} \epsilon-\frac{1}{48} B H_{M N P Q} \Gamma^{M N P Q} \epsilon & =\mathcal{O}\left(\alpha^{\prime 2}\right), \\
\frac{1}{3} B F_{M N P} \Gamma^{M N P} \epsilon+2 e^{2 \Phi} L F_{N} \Gamma^{N} \epsilon & =\mathcal{O}\left(\alpha^{\prime 2}\right),
\end{aligned}
$$

where $B H_{M N P Q}:=d H_{M N P Q}, B F_{M N R}:=3 \nabla_{[M} F_{N R]}$ and we have expanded the Einstein equation as $E=E^{(0)}+\alpha^{\prime} E^{(1)}+\mathcal{O}\left(\alpha^{\prime 2}\right)$ and similarly for $L \Phi$ the field equation for the dilaton. We have also used that $L H=L H^{(0)}+\mathcal{O}\left(\alpha^{\prime 2}\right), L F=L F^{(0)}+\mathcal{O}\left(\alpha^{\prime 2}\right)$ and $B F=B F^{(0)}+\mathcal{O}\left(\alpha^{\prime 2}\right)$. Of course $B H=\alpha^{\prime} B H^{(1)}+\mathcal{O}\left(\alpha^{\prime 2}\right)$. These integrability conditions assume a rather simple form whenever $B H=d H=0$ and $B F=0$ which we shall explore later.

\subsubsection{Spinors}

The general description of spinors in terms of forms is given in Appendix B. In particular, the Dirac spinors of $\operatorname{Spin}(9,1)$ are identified with $\Lambda^{*}\left(\mathbb{C}^{5}\right)$, and the positive chirality spinors are in $\Lambda^{\mathrm{ev}}\left(\mathbb{C}^{5}\right)$. A basis in $\Lambda^{\mathrm{ev}}\left(\mathbb{C}^{5}\right)$ is

$$
e_{i_{1} \ldots i_{k}}, \quad k=0,2,4 ; \quad e_{i_{1} \ldots i_{k} 5}, \quad k=1,3,
$$

where $i_{1}, \ldots, i_{k}=1, \ldots, 4$. This is a complex basis. To describe the Killing spinors of heterotic supergravity, one has to impose a reality condition. This is done using the reality map $r_{\mathrm{B}}=-\Gamma_{0} b *=\Gamma_{6789 *}$. As a result, a real basis is 


\section{Table 1}

In the columns are listed the number of invariant and therefore $\hat{\nabla}$-parallel spinors, their isotropy groups in $\operatorname{Spin}(9,1)$ and a basis in the space of invariant spinors, respectively. The basis of parallel spinors is always real. So if a complex spinor is given as a basis spinor it is understood that one should always take the real and imaginary parts.

\begin{tabular}{lll}
\hline$L$ & $\operatorname{Stab}\left(e_{1}, \ldots, e_{L}\right)$ & parallel $\epsilon$ \\
\hline 1 & $\operatorname{Spin}(7) \ltimes \mathbb{R}^{8}$ & $1+e_{1234}$ \\
2 & $S U(4) \ltimes \mathbb{R}^{8}$ & $1, i\left(e_{12}+e_{34}\right)$ \\
3 & $S p(2) \ltimes \mathbb{R}^{8}$ & $1, e_{12}$ \\
4 & $\times^{2} S U(2) \ltimes \mathbb{R}^{8}$ & $1, e_{12}, e_{13}+e_{24}$ \\
& & $1, e_{12}, e_{13}$ \\
5 & $S U(2) \ltimes \mathbb{R}^{8}$ & $1, e_{12}, e_{13}, e_{14}$ \\
6 & $U(1) \ltimes \mathbb{R}^{8}$ & $1+e_{1234}, e_{15}+e_{2345}$ \\
8 & $\mathbb{R}^{8}$ & $1, e_{15}$ \\
\hline 2 & $G_{2}$ & $1, e_{12}, e_{15}, e_{25}$ \\
4 & $S U(3)$ & $\Delta_{\mathbf{1 6}}^{+}$ \\
8 & $S U(2)$ & \\
16 & $\{1\}$ &
\end{tabular}

$$
\begin{aligned}
& e_{i_{1} \ldots i_{k}}+(-1)^{[k / 2]} \star e_{i_{1} \ldots i_{k}}, \quad i\left(e_{i_{1} \ldots i_{k}}-(-1)^{[k / 2]} \star e_{i_{1} \ldots i_{k}}\right), \quad k=0,2, \\
& e_{i 5}-\star e_{i 5}, \quad i\left(e_{i 5}+\star e_{i 5}\right),
\end{aligned}
$$

where $i_{1}<\cdots<i_{k}$ and star is the Hodge operation in $\Lambda^{*}\left(\mathbb{C}^{4}\right)$. The Killing spinors of Heterotic supergravity have real components in the basis (207).

\subsection{Solution of the Killing spinor equations for $d H=0$}

To begin, let us assume that the anomaly contribution to the Bianchi identity vanishes so $d H=0$. It is convenient to solve the KSEs in the order gravitino, gaugino and dilatino. One of the issues that arises is whether all the spinors that solve the gravitino KSE also solve the other two. We shall mainly focus on the description of the geometry of those backgrounds for which all solutions of the gravitino KSE also solve the other two. Then we shall describe some of the properties of the descendants, i.e. the backgrounds for which only some of the solutions of the gravitino KSE solve also the gaugino and dilatino KSEs.

\subsubsection{Solving the Gravitino KSE}

The gravitino Killing spinor equation is a parallel transport equation for a metric connection with skew-symmetric torsion, $\hat{\nabla}$. The (reduced) holonomy of $\hat{\nabla}$ for generic backgrounds is in $\operatorname{Spin}(9,1)$, i.e. in the same group as the gauge symmetry of the theory. The integrability condition of the gravitino KSE

$$
\hat{R}_{M N},{ }_{A B} \Gamma^{A B} \epsilon=0,
$$

implies that either the Killing spinors have a non-trivial isotropy group in $\operatorname{Spin}(9,1)$ or the spacetime is parallelizable, $\hat{R}=0$.

In the latter case, the spacetime is a Lorentzian group manifold. In particular, it decomposes, up to discrete identifications, to a suitable product of $S L(2, \mathbb{R}), \mathbb{R}^{n, 1}, \mathbb{R}^{n}, S U(2), S U(3)$ and $\mathrm{CW}_{n}(A) n \geq 4$ groups. The latter groups have been defined in Appendix C.2. The metric on the semi-simple group manifolds is required to be bi-invariant as the group manifold structure constants are identified with $H$ which is a 3-form.

Next suppose that the parallel spinors $\epsilon_{1}, \ldots, \epsilon_{L}$ have a non-trivial isotropy, or stability, group, Stab $\left(\epsilon_{1}, \ldots, \epsilon_{L}\right) \subset$ $\operatorname{Spin}(9,1)$. The spinors $\epsilon_{1}, \ldots, \epsilon_{L}$ are solutions of the gravitino KSE provided that the holonomy of $\hat{\nabla}$, hol $(\hat{\nabla})$, is contained in $\operatorname{Stab}\left(\epsilon_{1}, \ldots, \epsilon_{L}\right)$,

$$
\operatorname{hol}(\hat{\nabla}) \subseteq \operatorname{Stab}\left(\epsilon_{1}, \ldots, \epsilon_{L}\right) .
$$

The isotropy groups as well as representatives of the parallel spinors have been tabulated in Table 1 . There are two types of isotropy groups distinguished by whether their topology is compact or not. As in the previous theories we have described, the geometric properties of spacetime depend on the topology of the isotropy group.

The requirement (209) completely describes the solutions of the gravitino KSE. A consequence of (209) is that and the $\mathrm{k}$-form bi-linears $\tau$ of the $\hat{\nabla}$-parallel spinors defined in Appendix B are also parallel, i.e.

$$
\hat{\nabla}_{M} \tau_{N_{1} \ldots N_{k}}=0 \text {. }
$$

The forms $\tau$ are the fundamental forms of the group $\operatorname{Stab}\left(\epsilon_{1}, \ldots, \epsilon_{\ell}\right)$ viewed as the structure group of the spacetime. Therefore (210) can be interpreted as the conditions required for a manifold with structure group $\operatorname{Stab}\left(\epsilon_{1}, \ldots, \epsilon_{\ell}\right)$ to admit a compatible connection with skew-symmetric torsion. 


\subsubsection{Solving the Gaugino KSE}

The gaugino KSE in (201) can be viewed as the invariance condition of the spinor $\epsilon$ under infinitesimal Spin (9, 1) rotations generated by the 2-form field strength $F$. As such it is similar to the integrability condition of the gravitino KSE (208). Therefore either the solutions $\epsilon$ of the gaugino $\operatorname{KSE}$ have a non-trivial isotropy group in $\operatorname{Spin}(9,1)$ or $F=0$. In the former case, the restriction on $F$ is to lie in the Lie algebra of the isotropy group of spinors. These are the Lie algebras of the groups already tabulated in Table 1.

Suppose now that we have already solved the gravitino KSE and $\hat{\nabla}$ has holonomy $\operatorname{hol}(\hat{\nabla})=G$, where $G$ is one of the isotropy groups in Table 1 . The solutions of the gaugino KSE span a subspace of the solutions of the gravitino one. It turns out after some investigation [135] that this subspace can always be identified with the Lie algebra of one of the isotropy groups $K$ in Table 1 that is included in the Lie algebra of $G, \mathfrak{L i e} K \subseteq \mathfrak{L} \mathfrak{i} \in$.

To proceed one can either solve the gravitino KSE on the spinors with isotropy group $G$ and then separately solve the gaugino KSE by requiring $F$ to lie in the subalgebras of $\mathfrak{L i e} G$ contained in Table 1, or we can solve both gravitino and gaugino KSEs for the spinors invariant under $K$, i.e. the group that leaves invariant the solutions of the gaugino KSE. The geometry of spacetime with $\operatorname{hol}(\hat{\nabla})=K$ is less constrained from that with $\operatorname{hol}(\hat{\nabla})=G$. As a result the solutions to both gravitino and gaugino KSEs with $\operatorname{hol}(\hat{\nabla})=K$ include all those with the conditions $\operatorname{hol}(\hat{\nabla})=G$ and $F$ restricted to lie in $\mathfrak{L i e} K$. So without loss of generality, one can consider only the backgrounds for which all solutions of the gravitino KSE are also solutions of the gaugino KSE. This is the strategy that we shall adopt from here on.

\subsubsection{Solving the Dilatino KSE}

To solve the dilatino KSE, let us assume we have solved both the gravitino and gaugino ones and denote the space of their solutions with $\mathcal{K}_{G} \cdot \mathcal{K}_{G}$ is identified with the span of parallel spinors given in Table 1 . The space of solutions $\mathcal{K}_{D}$ of the dilatino KSE is a subspace in $\mathcal{K}_{G}, \mathcal{K}_{D} \subseteq \mathcal{K}_{G}$. To find all solutions of the KSEs of heterotic supergravity, one has to identify all subspaces of $\mathcal{K}_{G}$ which after solving the dilatino KSE give rise to a distinct spacetime geometry.

The method that has been introduced in [126] to solve this problem is to consider the subgroups $\Sigma\left(\mathcal{K}_{G}\right) \subset \operatorname{Spin}(9,1)$ which act almost effectively on $\mathcal{K}_{G}$. Clearly such transformations preserve the solutions of the gravitino and gaugino KSEs and can be used to choose $\mathcal{K}_{D}$ in $\mathcal{K}_{G}$. As all KSEs are covariant under $\operatorname{Spin}(9,1)$ gauge transformations, acting with elements of $\Sigma\left(\mathcal{K}_{G}\right)$ does not change the geometry of spacetime.

A detailed investigation of the action of $\Sigma\left(\mathcal{K}_{G}\right)$ on $\mathcal{K}_{G}$ reveals the following. If the isotropy group of the solutions of both the gravitino and gaugino KSEs is non-compact, then up to a $\Sigma\left(\mathcal{K}_{G}\right)$ transformation $\mathcal{K}_{D}$ can be identified with one of spaces of the invariant spinors in Table 1 contained in $\mathcal{K}_{G}$. Using the same argument as for the solution of the dilatino KSE, we can conclude that it suffices to consider only the cases for which all $\hat{\nabla}$-parallel spinors also solve the gaugino and dilatino KSEs. This is because these cases contain all other supersymmetric solutions of the theory for which only some of $\hat{\nabla}$-parallel spinors solve the other two KSEs.

It remains now to consider the case for which the isotropy group of the solutions of the gaugino and dilatino KSEs is compact. Again $\Sigma\left(\mathcal{K}_{G}\right)$ can be employed to choose $\mathcal{K}_{D}$ in $\mathcal{K}_{G}$. All the possibilities have been described in $[126,135,136]$. A further simplification can be made provided that $d H=0$. In such a case, the Bianchi identity of $\hat{R}$,

$$
\hat{R}_{A[B, C D]}=-\frac{1}{3} \hat{\nabla}_{A} H_{B C D},
$$

and the field equations imply that

$$
\hat{R}_{M N, P Q} \Gamma^{N} \Gamma^{P Q} \epsilon=\frac{1}{36} \hat{\nabla}_{M}\left(\Gamma^{N} \partial_{N} \Phi-\frac{1}{12} H_{N P Q} \Gamma^{N P Q}\right) \epsilon .
$$

Thus if $\epsilon$ solves the gravitino KSE but not the dilatino one, the gaugino KSE is covariantly constant with respect to $\hat{\nabla}$. This signals that the holonomy of $\hat{\nabla}$ reduces to a subgroup of the isotropy group of the $\hat{\nabla}$-parallel spinors. It can be shown that if there is a holonomy reduction, the pattern of reduction is

$$
G_{2} \supset S U(3) \supset S U(2) \supset\{1\}
$$

Thus it suffices to investigate the descendants for the cases in which the holonomy of $\hat{\nabla}$ is strictly $G_{2}, S U(3), S U(2)$ and $\{1\}$. Using this together with the classification of Lorentzian Lie algebras up to dimension six, one establishes the results described in Table 2. It turns out that the $G_{2}(N=1)$ and $S U(3)(N=1)$ solutions are included in the $\operatorname{Spin}(7) \times \mathbb{R}^{8}(N=1)$ backgrounds, and the $S U(3)(N=2)$ solutions are included in either the $G_{2}(N=2)$ or in the $S U(4) \ltimes \mathbb{R}^{8}(N=2)$ solutions.

\subsection{Geometry of backgrounds with non-compact holonomy}

From the results collected in Table 2 regarding the description of all independent solutions of the KSEs whose parallel spinors have a non-compact isotropy group, it suffices to describe the geometry of the backgrounds for which all parallel spinors are Killing, i.e. the parallel spinors solve all KSEs. So there are seven cases to consider all of which share common geometric properties. Because of this, to begin with we shall describe all of them together and only at the end provide formulae particular to each case. 


\section{Table 2}

In the columns are listed the holonomy groups that arise in the solution of the gravitino KSE and the number $N$ of supersymmetries, respectively. $\nearrow$ and denote the entries in Table 2 of [127] that are special cases of backgrounds for which all parallel spinors are Killing and those that do not occur, respectively.

\begin{tabular}{ll}
\hline $\operatorname{hol}(\hat{\nabla})$ & $N$ \\
\hline$S p i n(7) \ltimes \mathbb{R}^{8}$ & 1 \\
$S U(4) \ltimes \mathbb{R}^{8}$ & $\nearrow, 2$ \\
$S p(2) \ltimes \mathbb{R}^{8}$ & $\nearrow, \nearrow, 3$ \\
$\times^{2} S U(2) \ltimes \mathbb{R}^{8}$ & $\nearrow, \nearrow, \nearrow, 4$ \\
$S U(2) \ltimes \mathbb{R}^{8}$ & $\nearrow, \nearrow, \nearrow, \nearrow, 5$ \\
$U(1) \ltimes \mathbb{R}^{8}$ & $\nearrow, \nearrow, \nearrow, \nearrow, \nearrow, 6$ \\
$\mathbb{R}^{8}$ & $\nearrow, \nearrow, \nearrow, \nearrow, \nearrow, \nearrow,-, 8$ \\
\hline$G_{2}$ & 1,2 \\
$S U(3)$ & $1,2,-, 4$ \\
$S U(2)$ & $-, 2,-, 4,-, 6,-, 8$ \\
$\{1\}$ & $8,10,12,14,16$ \\
\end{tabular}

\subsubsection{Solving the Gravitino KSE}

To begin let us denote the isotropy group of the Killing spinors as $K \ltimes \mathbb{R}^{8}$, where $K$ is the compact subgroup, see Table 1 . In the spinorial geometry coframe $\left\{\mathbf{e}^{-}, \mathbf{e}^{+}, \mathbf{e}^{i}: i=1, \ldots, 8\right\}$, the form bilinears of the Killing spinors can be written as

$$
X=\mathbf{e}^{-}, \quad \tau=\mathbf{e}^{-} \wedge \phi,
$$

where $\mathbf{e}^{-}$is a null one-form and $\phi$ denotes collectively all the fundamental forms of $K$. As the Killing spinors $\epsilon$ satisfy $\hat{\nabla} \epsilon=0$, the form bilinears also satisfy

$$
\hat{\nabla} X=0, \quad \hat{\nabla} \tau=0 .
$$

As for the $N=4$ backgrounds of minimal $(1,0) d=6$ supergravity, the coframe $\left\{\mathbf{e}^{-}, \mathbf{e}^{+}, \mathbf{e}^{i}: i=1, \ldots, 8\right\}$ transforms under the isotropy group $K \ltimes \mathbb{R}^{8}$ as in (189), where now $O$ takes values in $K$ and $q$ in $\mathbb{R}^{8}$. There is no natural definition of the $\mathbf{e}^{+}$light-cone direction or that of the $\mathbf{e}^{i}$ directions "transverse" to the lightcone. Nevertheless the directions transverse to the lightcone can be identified as the orthogonal directions to $\mathbf{e}^{-}$, which are spanned by $\left\{\mathbf{e}^{-}, \mathbf{e}^{i}: i=1, \ldots, 8\right\}$, up to identifications along $\mathbf{e}^{-}$. So again the bundle transverse to the lightcone is $\mathcal{T}=I^{\perp} / I$, where $I$ is the trivial sub-bundle of $T^{*} M$ with fibre spanned by $\mathbf{e}^{-}$and $I^{\perp}$ is its orthogonal complement in $T^{*} M$.

Let us now consider the condition (215). First, one finds that

$$
\hat{\nabla}_{A} X=0 \Longleftrightarrow d X=i_{X} H, \quad \mathcal{L}_{X} g=0 .
$$

Thus the vector field $X$ is Killing. As $d H=0$, it leaves $H$ invariant as well. The same applies for the dilaton $\Phi$ as well.

To continue observe that in the coframe we have chosen, the restriction of $\hat{\nabla}$ to have holonomy contained in $K \ltimes \mathbb{R}^{8}$ implies that $\hat{\Omega}_{A,+B}=0$. Using this, the second condition in (215) can be rewritten as

$$
\begin{aligned}
\nabla_{+} \phi_{j_{1} \ldots j_{k}}= & (-1)^{k} \frac{k}{2} H_{+}{ }^{i}\left[j_{1} \phi_{\left.j_{2} \ldots j_{k}\right] i},\right. \\
\nabla_{-} \phi_{j_{1} \ldots j_{k}}= & (-1)^{k} \frac{k}{2} H_{-}{ }^{i}\left[j_{1} \phi_{\left.j_{2} \ldots j_{k}\right] i},\right. \\
& \hat{\nabla}_{i} \phi_{j_{1} \ldots j_{k}}=0 .
\end{aligned}
$$

To investigate the geometric significance of (217) observe that $K \subset \operatorname{Spin}(8)$ and as the Lie algebra of $\operatorname{Spin}(8)$ is $\mathfrak{s p i n}(8)=$ $\Lambda^{2}\left(\mathbb{R}^{8}\right)$, the Lie algebra of $K, \mathfrak{k}$, is a subspace of $\Lambda^{2}\left(\mathbb{R}^{8}\right), \mathfrak{k} \subset \Lambda^{2}\left(\mathbb{R}^{8}\right)$. Therefore we can write $\Lambda^{2}\left(\mathbb{R}^{8}\right)=\mathfrak{k} \oplus \mathfrak{k}^{\perp}$, where $\mathfrak{k}^{\perp}$ is the orthogonal complement of $\mathfrak{k}$ in $\Lambda^{2}\left(\mathbb{R}^{8}\right)$. This decomposition of vector spaces leads to a decomposition of $\Lambda^{2}(\mathcal{T})$ as $\Lambda^{2}(\mathcal{T})=\Lambda_{\mathfrak{k}}^{2} \oplus \Lambda_{\mathfrak{k} \perp}^{2}$, where $\Lambda_{\mathfrak{k}}^{2}$ and $\Lambda_{\mathfrak{k} \perp}^{2}$ have typical fibre $\mathfrak{k}$ and $\mathfrak{k}^{\perp}$, respectively.

Next let us focus on the first equation in (217). It is clear that this condition does not depend on $\left.i_{X} H\right|_{\Lambda_{\mathrm{f}}^{2}}$ and expresses $\left.i_{X} H\right|_{\Lambda_{\mathfrak{k}^{\perp}}^{2}}$ in terms of the covariant derivative of $\phi$ along $X$. However, $\left.i_{X} H\right|_{\Lambda_{\mathfrak{k} \perp}^{2}}$ is also expressed in terms of the $\Lambda_{\mathfrak{k}^{\perp}}^{2}$ component of $d \mathbf{e}^{-\underline{\perp}}$ in (216). Therefore consistency requires that schematically

$$
\left.\left(d \mathbf{e}^{-}\right)\right|_{\Lambda_{\mathfrak{t}^{\perp}}^{2}}=\left.\left(\nabla_{X} \phi\right)\right|_{\Lambda_{\mathfrak{t}^{\perp}}^{2}},
$$

which is interpreted as a condition on the geometry.

Similarly, one can see from the second condition in (217) that $\left.\left(H_{-}\right)\right|_{\Lambda_{\mathfrak{k}}^{2}}$ is not restricted by the gravitino KSE while $\left.\left(H_{-}\right)\right|_{\Lambda_{\mathfrak{k} \perp}^{2}}$ is expressed in terms of the $\nabla_{-} \phi$.

It remains to investigate the last condition in (217). This can be analysed as though it is examined on an 8-dimensional manifold with tangent space $\mathcal{T}$ admitting a $K$-structure compatible with a connection with skew-symmetric torsion. The 
end result depends on the $K$ structure at hand and it may or may not give additional conditions on the geometry. In all cases, the component of $H$ along $\Lambda^{3}(\mathcal{T})$ is entirely determined in terms of the geometry.

Furthermore, notice that a consequence of (215)-(217) is that the only not trivial component of $\mathcal{L}_{X} \tau$ is

$$
\left.\mathcal{L}_{X} \tau_{-i_{1} \ldots i_{k}}=k\left(i_{X} H\right)^{j}{ }_{\left[i_{1}\right.} \tau_{i_{2} \ldots i_{k}}\right] j-\cdot
$$

Thus the form bilinears $\tau$ are invariant under the vector field $X, \mathcal{L}_{X} \tau=0$, if $\left.i_{X} H\right|_{\Lambda_{\mathfrak{k}}^{\perp}} ^{2}=0$. This turns out to be useful in the investigation of geometry of some backgrounds.

\subsubsection{Solving the Gaugino KSE}

The gaugino Killing spinor equation implies that

$$
F \in \Lambda_{\mathfrak{k} \oplus \mathbb{R}^{8}}^{2} \otimes \mathfrak{h},
$$

where $\mathfrak{h}$ is the Lie algebra of the gauge group. This means that there is a 1-form $P$ and a 2 -form $Q$ along the transverse directions with values in the Lie algebra of the gauge group such that

$$
F=\mathbf{e}^{-} \wedge P+Q,
$$

where $Q \in \Lambda_{\mathfrak{k}}^{2} \otimes \mathfrak{h}$. The condition on $Q$ is a standard instanton like condition which arises on manifolds with structure group $K$. These will be described later for the individual cases that occur.

\subsubsection{Solving the Dilatino KSE}

It remains to investigate the conditions that arise on the fields form the dilatino KSE. In all cases, the dilatino KSE implies that

$$
i_{X} d \Phi=0,\left.\quad d X\right|_{\Lambda_{\mathbf{k}^{\perp}}^{2}}=0, \quad 2 \partial_{i} \Phi-\left(\tilde{\theta}_{\phi}\right)_{i}-H_{-+i}=0 .
$$

So the dilaton is invariant under the action of $X$. The second condition is equivalent to requiring that $d \mathbf{e}^{-} \in \Lambda_{\mathfrak{k} \oplus \mathbb{R}^{8}}^{2}$ and it is exactly the same condition as that which arises from the gaugino KSE on the field strength $F$. Thus (216), (219) and (222) imply that

$$
\mathcal{L}_{X} \tau=0
$$

and that the geometric condition (218) is automatically satisfied. The third condition in (222) is a geometric condition which relates the Lee form $\tilde{\theta}_{\phi}$ of $\phi$ to the dilaton $\Phi$ and a component of $H$. The expression of the Lee form depends on $\phi$ and it will be given later during the investigation of individual cases.

The dilatino Killing spinor equation implies additional conditions to those given in (222). However, these depend on the choice of holonomy group $K \ltimes \mathbb{R}^{8}$.

The conditions (216), (222) and (223) are common for all cases and we shall refer them as universal. It remains to explain the non-universal conditions on the spacetime geometry which depend on the case under consideration. These arise from both the $\hat{\nabla} \tau=0$ condition restricted along the directions transverse to the light-cone and from the dilatino KSE.

\subsubsection{Fields}

After solving the KSEs, one can demonstrate that in all cases the metric and 3-form field strength can be written as

$$
\begin{aligned}
d s^{2} & =2 \mathbf{e}^{-} \mathbf{e}^{+}+\delta_{i j} \mathbf{e}^{i} \mathbf{e}^{j}, \\
H & =d \mathbf{e}^{-} \wedge \mathbf{e}^{+}+\mathbf{e}^{-} \wedge(\tilde{h}+\tilde{k})+\tilde{H},
\end{aligned}
$$

where $\tilde{h} \in \Lambda_{\mathfrak{k}}^{2}, \tilde{k} \in \Lambda_{\mathfrak{k} \perp}^{2}$ and $\tilde{H}=\left.H\right|_{\mathcal{T}}=\frac{1}{3 !} H_{i j k} \mathbf{e}^{i} \wedge \mathbf{e}^{j} \wedge \mathbf{e}^{k}$. The $\tilde{h}$ component of $H$ is not restricted by the KSEs and it remains arbitrary. However the restriction $\tilde{h} \in \Lambda_{\mathfrak{k}}^{2}$ imposes an instanton like condition on $\tilde{h}$ associated to each $K$. The $\tilde{k}$ and $\tilde{H}$ components of $H$ are always expressed in terms of the geometry of spacetime but the precise relation depends on the choice of the holonomy group. In what follows, we shall not express $\tilde{k}$ in terms of the geometry as it does not appear in local calculations after an appropriate choice of a coframe.

\subsection{5. $\operatorname{Spin}(7) \ltimes \mathbb{R}^{8}, N=1$}

The form bilinears are $\mathbf{e}^{-}$and $\tau=\mathbf{e}^{-} \wedge \phi$, where $\phi$ is the self-dual fundamental 4-form of $\operatorname{Spin}(7)$, see [115] for an expression of $\phi$ in the spinorial geometry coframe. The instanton condition on the $\tilde{h}$ component of $H$, the $Q$ component of $F$ and the $\left.d \mathbf{e}^{-}\right|_{\Lambda_{\mathcal{T}}^{2}}$ component of $d \mathbf{e}^{-}$to be in $\Lambda_{\mathfrak{s p i n}(7)}^{2}$ is

$$
\tilde{h}_{i j}=\frac{1}{2} \phi_{i j}{ }^{k l} \tilde{h}_{k l},
$$

and similarly for the other two fields. 
The remaining conditions of the gravitino KSE give

$$
\tilde{H}=-\star_{8} \tilde{d} \phi+\star_{8}\left(\tilde{\theta}_{\phi} \wedge \phi\right),
$$

where the Lee form of $\phi$ is

$$
\tilde{\theta}_{\phi}=-\frac{1}{6} \star_{8}\left(\star_{8} \tilde{d} \phi \wedge \phi\right),
$$

the Hodge duality operation is that in $\mathcal{T}$, and $\tilde{d}$ denotes the exterior derivative like operation defined on 1-forms restricted to $\mathcal{T}$ as $\tilde{d} \tau=\left(\partial_{i} \tau_{j}-\Omega_{i,}{ }^{k} \tau_{k}\right) \mathbf{e}^{i} \wedge \mathbf{e}^{j}$ and similarly extended to k-forms restricted to $\mathcal{T}$.

The dilatino KSE does not give any additional conditions to those already presented in (222). The Lee form that appears in the last equation of (222) is (227).

It is clear that $\tilde{H}$ can be expressed in terms of the fundamental $\operatorname{Spin}(7)$ form $\phi$. The expression is similar to that for a $d=8$ Euclidean signature manifold with a Spin(7) structure and compatible connection with skew-symmetric torsion [137]. There are no further geometric conditions implied by the gravitino KSE as any 8-dimensional manifold with a Spin(7) structure admits a unique such connection.

\subsection{6. $S U(4) \ltimes \mathbb{R}^{8}, N=2$}

The $\hat{\nabla}$-parallel forms are $\mathbf{e}^{-}, \mathbf{e}^{-} \wedge \omega_{I}$ and $\mathbf{e}^{-} \wedge \chi$, where $\omega_{I}$ and $\chi$ are the Hermitian 2-form associated with an almost complex structure $I$ on $\mathcal{T}$ and the $(4,0)$ fundamental forms of $S U(4)$, respectively. The normalization of the fundamental forms chosen is $\frac{1}{4 !} \wedge^{4} \omega_{I}=2^{-4} \chi \wedge \bar{\chi}=d \operatorname{vol}_{\mathcal{T}}$, where $d \operatorname{vol}_{\mathcal{T}}$ is the volume form of the metric on $\mathcal{T}$.

The condition that $h, Q$ and $\left.d \mathbf{e}^{-}\right|_{\mathcal{T}}$ must be in $\Lambda_{\mathfrak{s u}(4)}^{2}$ reads

$$
\tilde{h}_{k l} I^{k}{ }_{i}{ }^{l}{ }_{j}=\tilde{h}_{i j}, \quad \tilde{h}_{i j} \omega_{I}^{i j}=0,
$$

and similarly for the other two fields.

The gravitino KSE together with the second condition in (230) gives

$$
\tilde{H}=-\tilde{i}_{\tilde{I}} \tilde{d} \omega_{I}=\star_{8}\left(\tilde{d} \omega_{I} \wedge \omega_{I}\right)-\frac{1}{2} \star_{8}\left(\tilde{\theta}_{\omega_{I}} \wedge \omega_{I} \wedge \omega_{I}\right) .
$$

The expression for $\tilde{H}$ is as that for complex $d=8$ manifolds with either a $U(4)$ or an $S U(4)$ structure and compatible connection with skew-symmetric torsion. Manifolds with $S U(n)$ structures have extensively been explored in the literature, see e.g. [131,132,138-143].

The additional geometric conditions implied by the KSEs are

$$
\tilde{\theta}_{\omega_{I}}=\tilde{\theta}_{\operatorname{Re} \chi}, \quad \tilde{\mathcal{N}}(I)=0
$$

where

$$
\tilde{\theta}_{\omega_{I}}=-\star_{8}\left(\star_{8} \tilde{d} \omega_{I} \wedge \omega_{I}\right), \quad \tilde{\theta}_{\operatorname{Re} \chi}=-\frac{1}{4} \star_{8}\left(\star_{8} \tilde{d} \operatorname{Re} \chi \wedge \operatorname{Re} \chi\right),
$$

are the Lee forms of $\omega_{I}$ and Re $\chi$, respectively, and $\tilde{\mathcal{N}}$ is the Nijenhuis tensor of $I$ restricted along the transverse directions. The vanishing condition of the Nijenhuis tensor is equivalent to requiring that $\tilde{H}$ is a $(2,1)$ - and (1,2)-form with respect to $I$.

The vanishing of the Nijenhuis tensor is implied by the dilatino KSE. A consequence of this is that the complexified tangent bundle $T M \otimes \mathbb{C}$ of the spacetime admits a Lorentzian complex structure, i.e. it is a Robinson manifold [144]. To see this observe that the 1-forms $\left(\mathbf{e}^{-}, \mathbf{e}^{\alpha}\right)$, where $I\left(\mathbf{e}^{\alpha}\right)=i \mathbf{e}^{\alpha}$, induce an integrable distribution on $T M \otimes \mathbb{C}$.

On the other hand, the equality of the two Lee forms is required for the existence of a connection with skew-symmetric torsion compatible with an $S U(4)$ structure. This geometric condition arises because $\tilde{H}$ is uniquely determined in (229) in terms of $I$ and $\omega_{I}$. However, it is also required that $\chi$ must be covariantly constant with respect to $\hat{\nabla}$ along $\mathcal{T}$. This also expresses some of the components of $\tilde{H}$ in terms of the metric and $\chi$. So the compatibility of $\left.\hat{\nabla} \omega_{I}\right|_{\mathcal{T}}=0$ and $\left.\hat{\nabla} \chi\right|_{\mathcal{T}}=0$ gives the equality of the two Lee forms in (231).

\subsubsection{The geometry of solutions with $3 \leq N \leq 6$}

The holonomy of $\hat{\nabla}$ for all these cases is $\operatorname{Sp}(2) \ltimes \mathbb{R}^{8}, N=3 ; \times^{2} S U(2) \ltimes \mathbb{R}^{8}, N=4 ; S U(2) \ltimes \mathbb{R}^{8}, N=5$; and $U(1) \times \mathbb{R}^{8}$, $N=6$. The geometry [126] of all these solutions can be summarized as follows. The $\hat{\nabla}$-parallel forms which are relevant for the description of the spacetime geometry are $\mathbf{e}^{-}$and $\mathbf{e}^{-} \wedge \omega_{r}$, where $\omega_{r}=\tilde{\omega}_{r}, r=1, \ldots N-1$, are Hermitian forms on $\mathcal{T}$. The associated (almost) complex structures $I_{r}$ satisfy the relations of the standard basis of the Clifford algebra Cliff( $\mathbb{R}^{N-1}$ ) equipped with a negative definite inner product. For example in the $S p(2) \ltimes \mathbb{R}^{8}$ case, the associated Clifford algebra is $C$ liff $\left(\mathbb{R}^{2}\right)$. Therefore there are two (almost) complex structures $I_{1}$ and $I_{2}$ that satisfy the relations $I_{1}^{2}=I_{2}^{2}=-\mathbf{1}$ and $I_{1} I_{2}+I_{2} I_{1}=0$. The third (almost) complex structure that arises in the description of manifolds with holonomy $\operatorname{Sp}(2)$ is given by $I_{3}=I_{1} I_{2}$.

Turning now to the description of the fields and geometry for these solutions, the condition that $\tilde{h}, F$ and $\left.d \mathbf{e}^{-}\right|_{\mathcal{T}}$ must lie in $\Lambda_{\mathfrak{k}}^{2}$ can be written as

$$
\tilde{h}_{k l}\left(I_{r}\right)^{k}{ }_{i}\left(I_{r}\right)_{j}^{l}=\tilde{h}_{i j}, \quad \text { no summation over } r,
$$

and similarly for the other two fields. 
Now $\tilde{H}$ can be given as in (229) with respect to any of the (almost) complex structures $I_{r}$. Similarly, the last condition in (222) is valid with respect to the Lee form $\theta_{r}$ of each of the Hermitian forms $\omega_{r}$. Therefore one finds the geometric conditions

$$
\begin{aligned}
& i_{I_{I}} \tilde{d} \omega_{r}=i_{I_{s}} \tilde{d} \omega_{s}, \quad r \neq s, \text { no summation over } r \text { or } s, \\
& \tilde{\theta}_{r}=\tilde{\theta}_{s}, \quad r \neq s .
\end{aligned}
$$

The expression for the Lee form $\tilde{\theta}_{r}$ of $\omega_{r}$ is the same as that for $\omega_{I}$ in (231). The only additional geometric condition that arises is

$$
\tilde{\mathcal{N}}\left(I_{r}\right)=0,
$$

which is the integrability condition of $I_{r}$. The above conditions we have described on the geometry of the spacetime can be derived from those we have stated for the $S U(4) \ltimes \mathbb{R}^{8}$ backgrounds but now require that they are valid for each of the $I_{r}$ complex structures.

\subsection{8. $\mathbb{R}^{8}, N=8$}

It remains to give the conditions imposed by the KSEs on the fields of backgrounds with holonomy $\mathbb{R}^{8}$ which preserve $N=8$ supersymmetries. These are

$$
\mathbf{e}^{-} \wedge d \mathbf{e}^{-}=0, \quad \tilde{H}=0,2 \partial_{i} \Phi-H_{-+i}=0, \quad Q=0 .
$$

Observe in particular that $\tilde{h}=0$ as well. Furthermore $F=\mathbf{e}^{-} \wedge P$ and hence it is null.

\subsubsection{Field equations from KSES}

Some of the field equations are implied by the Killing spinor equations. It turns out that in all the $K \ltimes \mathbb{R}^{8}$ cases, the field equations of the theory are implied by the KSEs and the Bianchi identities, $B H \equiv d H=0$ and $B F=0$, provided that in addition the following components of the field equations (203)

$$
E_{--}=0, L H_{-A}=0, L F_{-}=0,
$$

are also imposed.

To see this consider the first integrability condition in (205). Taking the (Dirac) inner product with $\epsilon$, one finds

$$
2 E_{A+}+e^{2 \Phi} L H_{A+}=0 \text {. }
$$

On the other hand, acting on the first integrability condition with $\left(2 E_{A C}+e^{2 \Phi} L H_{A C}\right) \Gamma^{C}$, one finds that

$$
\left(2 E_{A B}+e^{2 \Phi} L H_{A B}\right)\left(2 E_{A}{ }^{B}+e^{2 \Phi} L H_{A}{ }^{B}\right)=0 \text {, no summation over } A,
$$

as $\epsilon \neq 0$. Combining (237) and (238), one can derive the first two conditions in (236). A similar argument using the remaining integrability conditions in (205) establishes the last condition in (236) as well. It should be noted that the set of field equations that have to be imposed in addition to the KSEs to find solutions can be further refined compared to those in (236) for backgrounds that preserve $N>1$ supersymmetries.

\subsubsection{Special local coordinates}

Local coordinates to describe the geometry of $K \ltimes \mathbb{R}^{8}$ backgrounds can be chosen in a way similar to that we have described for the $S p(1) \ltimes \mathbb{H}$ solutions of $\mathcal{N}=(1,0) d=6$ supergravity in Section 6.2.2. After adapting coordinates to the Killing vector field $X$ as $X=\partial_{u}$ and using a coframe rotation as in (189), a coframe can be chosen locally as

$$
\mathbf{e}^{-}=W\left(d v+m_{I} d y^{I}\right), \quad \mathbf{e}^{+}=d u+V d v+n_{l} d y^{I}, \quad \mathbf{e}^{i}=e_{I}^{i} d y^{I} .
$$

Furthermore, as the metric, $H$ and the fundamental forms are invariant under the action of $X$, all component of the coframe can be chosen to be independent of $u$ though they can depend on the $y$ and $v$ coordinates. In such a case, the 3-form flux $H$ can be rewritten as

$$
H=d\left(\mathbf{e}^{-} \wedge \mathbf{e}^{+}\right)+\mathbf{e}^{-} \wedge \tilde{w}+\tilde{H},
$$

for some $\tilde{w} \in \Lambda_{\mathfrak{k}}$, generally $\tilde{w} \neq \tilde{h}$, which is not specified by the KSEs. Note that $\tilde{w}$ satisfies the same instanton like conditions as $\tilde{h}$. The above expression of $H$ is more helpful as it is more straightforward to impose the Bianchi identity.

As we have seen in the $\mathbb{R}^{8} N=8$ case, $\mathbf{e}^{-} \wedge d \mathbf{e}^{-}=0$. Therefore there is a function $h=h(v, y)$ such that $\mathbf{e}^{-}=h d v$. This is useful for the classification of half supersymmetric solutions [145] which will be described below. 


\subsection{Geometry of backgrounds with compact holonomy}

\subsubsection{Gravitino KSE}

The $\hat{\nabla}$-parallel forms on the spacetime $M$ for the compact holonomy groups $K$ in Table $1, \operatorname{hol}(\hat{\nabla}) \subseteq K$, are

$$
\lambda^{a}=\mathbf{e}^{a}, \quad \phi=\frac{1}{k !} \phi_{i_{1} \ldots i_{k}} \mathbf{e}^{i_{1}} \wedge \cdots \wedge \mathbf{e}^{i_{k}},
$$

where $\left\{\mathbf{e}^{a}, \mathbf{e}^{i}\right\}$ is a spinorial geometry coframe, $\lambda^{a}$ are 1 -forms, and $\phi$ represents collectively the fundamental forms of $K$. In particular, there is always one time-like $\hat{\nabla}$-parallel 1 -form $\lambda^{a}$, and 2,3 and 5 space-like 1 -forms for $K=G_{2}, S U(3)$ and $S U(2)$ in Table 1, respectively. Furthermore $i_{a} \phi=0$, where $i_{a}$ denotes inner derivation with respect to (the associated vector field of) $\lambda^{a}$. The change of notation from $X$ to $\lambda$ will become apparent below.

Moreover, one has that

$$
d s^{2}=\eta_{a b} \lambda^{a} \lambda^{b}+d \tilde{s}^{2}, \quad d \tilde{s}^{2}=\delta_{i j} \mathbf{e}^{i} \mathbf{e}^{j}
$$

where $\eta$ is a constant Lorentzian signature metric as the inner product of $\lambda^{a}$ is constant because they are parallel with respect to the metric connection $\hat{\nabla}$.

The condition $\hat{\nabla} \lambda^{a}=0$ implies that

$$
d \lambda^{a}=\eta^{a b} i_{b} H, \quad \mathcal{L}_{a} g=0 .
$$

Therefore the $i_{a} H$ components of $H$ are determined in terms of $d \lambda^{a}$ and $\lambda^{a}$ are Killing vector fields.

Suppose that $\phi$ is an additional $\hat{\nabla}$-parallel form, then the condition $\hat{\nabla} \phi=0$ evaluated along the coframe $\left(\lambda^{a}, e^{i}\right)$ gives

$$
\begin{aligned}
\nabla_{a} \phi_{j_{1} \ldots j_{k}} & =\frac{k}{2}(-1)^{k} H_{a}{ }^{i}\left[j_{1} \phi_{\left.j_{2} \ldots j_{k}\right] i},\right. \\
\hat{\nabla}_{i} \phi_{j_{1} \ldots j_{k}} & =0,
\end{aligned}
$$

where we have used that $i_{a} \phi=0$.

To continue, first observe that $T M=\Xi \oplus \mathcal{T}$, where $\Xi$ is spanned by the parallel, and thus nowhere vanishing, vector fields $\lambda^{a}$, and $\mathcal{T}$ is the orthogonal complement of $\Xi$ with respect to the metric, where we again denote the 1-forms $\lambda^{a}$ and the associated vector fields with the same symbol. As in the null case, we refer to $\mathcal{T}$ as the "transverse space". As the structure group of $M$ has reduced to $K, \Lambda^{2}(\mathcal{T})$ decomposes as $\Lambda^{2}(\mathcal{T})=\Lambda_{\mathfrak{k}}^{2} \oplus \Lambda_{\mathfrak{k} \perp}^{2}$, k is the Lie algebra of $K$. The argument for this decomposition has already been presented in the null case. Next observe that $\left.i_{a} H\right|_{\Lambda_{\mathfrak{k} \perp}^{2}}$ is determined in terms of the geometry as a consequence of the first equation in (244). However the first equation in (243) also gives $\left.i_{a} H\right|_{\Lambda_{\mathfrak{p}}^{2}} ^{2}$ in terms of $\left.d \lambda^{a}\right|_{\Lambda_{\mathfrak{k} \perp}^{2}}$. As a result, consistency requires that we have a restriction on the geometry which schematically can be written as

$$
\left.d \lambda^{a}\right|_{\Lambda_{\mathfrak{k} \perp}^{2}}=\left.\eta^{a b}\left(\nabla_{b} \phi\right)\right|_{\Lambda_{\mathfrak{k} \perp}^{2}}
$$

It remains to investigate the last condition in $\left.(244)(\hat{\nabla} \phi)\right|_{\mathcal{T}}=0$. This condition can be solved as that which arises for manifolds with a $K$-structure compatible with a connection with skew-symmetric torsion and tangent space $\mathcal{T}$. In all cases, $\tilde{H}=\left.H\right|_{\mathcal{T}}$ is entirely determined in terms of the geometry. We shall not give further details here but we describe the final result separately for each case.

Using $\hat{\nabla} \lambda^{a}=\hat{\nabla} \phi=0$, one can also compute the Lie derivative of $\lambda^{a}$ and $\phi$ along $\lambda^{a}$ to find

$$
\begin{aligned}
{\left[\lambda^{a}, \lambda^{b}\right] } & =-H^{a b}{ }_{c} \lambda_{c}-H^{a b i} \mathbf{e}_{i} \\
\mathcal{L}_{a} \phi_{i_{1} i_{2} \ldots i_{k}} & =k(-1)^{k} H_{a}{ }^{j}\left[i_{1} \phi_{\left.i_{2} \ldots i_{k}\right] j},\right. \\
\mathcal{L}_{a} \phi_{b i_{1} \ldots i_{k-1}} & =(-1)^{k} H_{a}{ }_{b}{ }_{b} \phi_{i_{1} \ldots i_{k-1} j} .
\end{aligned}
$$

To analyse these conditions observe that if the span of $\lambda_{a}$ closes under Lie brackets, i.e. symbolically [ $\Xi$, $\Xi$ ] $\Xi$, then $H_{a b i}=0$. Also $\phi$ is invariant under the action of the vector fields $\lambda^{a}$, i.e. $\mathcal{L}_{a} \phi=0$, provided that $\left.i_{a} H\right|_{\Lambda_{\mathfrak{k}}^{\perp}}$ vanishes and $[\Xi, \Xi] \subseteq \Xi$. Moreover observe from (243) that if $d H=0$, then $\mathcal{L}_{a} H=0$.

In what follows, we shall assume that the algebra of vector field bilinears $\lambda^{a}$ closes. One reason for this is the results of [146] where it has been demonstrated that the Killing superalgebras of supersymmetric backgrounds close on the vector generators constructed as Killing spinor bilinears. Another reason is that if the commutator of two such vector fields does not close, it is nevertheless $\hat{\nabla}$-parallel and so the holonomy of $\hat{\nabla}$ reduces further yielding more parallel spinors. So if we insist that the number of parallel spinors is fixed, we are required to take $H_{a b i}=0$

Let $\mathfrak{g}$ be the Lie algebra of the Killing vector fields $\lambda^{a}$. The structure constants of $\mathfrak{g}$ are given by $H_{a b c}$ and as this is skewsymmetric the metric $\eta$ must be bi-invariant. The Lorentzian Lie algebras $\mathfrak{g}$ up to dimension 6 that are relevant here have been tabulated in Table 3. There are many ways to utilize the above data to write the spacetime metric and $H$. The most economical way is to assume that the infinitesimal action generated by the vector fields $\lambda^{a}$ can be integrated to a free action 
Table 3

In the first column, the compact isotropy groups of spinors are stated. In the second column, the number of 1 -form bilinear is given. In the third column, the associated Lorentzian Lie algebras are exhibited. The structure constants of the 6-dimensional Lorentzian Lie algebras of the $S U(2)$ case are self-dual.

\begin{tabular}{lll}
\hline $\operatorname{Stab}\left(\epsilon_{1}, \ldots, \epsilon_{L}\right)$ & $1-$ forms & $\mathfrak{L} \mathfrak{i} \mathfrak{c} G$ \\
\hline$G_{2}$ & 3 & $\mathbb{R}^{1,2}, \mathfrak{s l}(2, \mathbb{R})$ \\
$\operatorname{SU}(3)$ & 4 & $\mathbb{R}^{1,3}, \mathfrak{s l}(2, \mathbb{R}) \oplus \mathbb{R}, \mathfrak{s u}(2) \oplus \mathbb{R}, \mathfrak{c w}_{4}$ \\
$\operatorname{SU}(2)$ & 6 & $\mathbb{R}^{1,5}, \mathfrak{s l}(2, \mathbb{R}) \oplus \mathfrak{s u}(2), \mathfrak{c w}_{6}$ \\
\hline
\end{tabular}

by a group $G$ with Lie algebra $\mathfrak{g}$. Then the spacetime is a principal bundle, $M=P(G, B, \pi)$, equipped with a principal bundle connection, $\lambda^{a}$. In this case, one finds

$$
\begin{aligned}
d s^{2} & =\eta_{a b} \lambda^{a} \lambda^{b}+\delta_{i j} \mathbf{e}^{i} \mathbf{e}^{j}, \\
H & =\frac{1}{3} \eta_{a b} \lambda^{a} \wedge d \lambda^{b}+\frac{2}{3} \eta_{a b} \lambda^{a} \wedge \mathcal{F}^{b}+\tilde{H}, \quad \tilde{H}=\left.H\right|_{\mathcal{T}},
\end{aligned}
$$

where

$$
\mathcal{F}^{a}:=\frac{1}{2} H^{a}{ }_{i j} \mathbf{e}^{i} \wedge \mathbf{e}^{j}=d \lambda^{a}-\frac{1}{2} H^{a}{ }_{b c} \lambda^{b} \wedge \lambda^{c},
$$

is the curvature of the principal bundle. As $\mathcal{L}_{a} \tilde{H}=0$ and $i_{a} \tilde{H}=0, \tilde{H}$ is the pull back of a 3 -form on the base space $B$. Therefore, $H$ is the sum of the Chern-Simons form of the principal bundle connection $\lambda$ and the pull-back of a 3-form on $B$ which we again denote with $\tilde{H}$. As a consequence,

$$
d H=\eta_{a b} \mathcal{F}^{a} \wedge \mathcal{F}^{b}+d \tilde{H} .
$$

As $d H=0$, the right-hand-side of the equation above must vanish. This condition resembles the anomalous Bianchi identity of $H$ in (200), where $H$ is replaced by $\tilde{H}$, and the curvature of the spacetime $\tilde{R}$ and that of the gauge connection $F$ are replaced by the curvature of the principal fibration $\mathcal{F}$.

\subsubsection{Gaugino KSE}

The gaugino Killing spinor equation implies that

$$
F \in \Lambda_{\mathfrak{k}}^{2} \otimes \mathfrak{h},
$$

where $\mathfrak{h}$ is the Lie algebra of the gauge group. This is an instanton like condition associated with the holonomy group $K$. As this depends on $K$, it will be stated in each case separately.

\subsubsection{Dilatino KSE}

To simplify the description of the solutions of the dilatino KSE, let us assume that all solutions of the gravitino and gaugino KSEs also solve dilatino one. There are descendants for compact holonomy groups and these have been investigated in detail in [136]. Under this assumption, the dilatino KSE implies the universal conditions

$$
i_{a} d \Phi=0, \quad 2 \partial_{i} \Phi-\left(\tilde{\theta}_{\phi}\right)_{i}=0,
$$

where $\tilde{\theta}_{\phi}$ is the Lee form of $\phi$ which will be given for each case separately. Therefore the dilaton $\Phi$ is invariant under the action of vector field bilinears and therefore a function of the base space $B$ of the spacetime fibration. In all cases that the Lee form of a $\phi$ satisfies (251), $\phi$ and also its Lee form $\tilde{\theta}_{\phi}$, are pull-backs of a form and a Lee form on $B$, respectively. So the second condition in (251) implies that $B$ is "conformally balanced" with respect to $\phi$. There are additional conditions that are implied by the dilatino KSE but they depend on the holonomy group $K$.

\subsubsection{Field equations from KSEs}

All the field equations of backgrounds with compact holonomy group are implied by the KSEs after imposing the Bianchi identities of the theory. The proof of this is similar to that described in Section 7.3.9 for solutions with non-compact holonomy group. The main difference is now that there is a time-like $\hat{\nabla}$-parallel vector field bilinear while in the non-compact cases the bilinear is null. Thus to find solutions, one has to impose $d H=0$ and $B F=0$, where $d H$ is given in (249).

\subsection{5. $G_{2}, N=2$}

The $\hat{\nabla}$-parallel forms are $\lambda^{a}, a=0,1,2$ which span $\Xi$, and $\varphi \in \Lambda^{3}(\mathcal{T})$, where $\varphi$ is the fundamental $G_{2}$ form, see [115] for an expression of $\varphi$ in the spinorial geometry coframe. In addition to the conditions in (251), the dilatino KSE implies that

$$
\mathcal{F} \in \Lambda_{\mathfrak{g}_{2}}^{2} \otimes \mathfrak{g}, \quad \epsilon^{a b c} H_{a b c}+H_{i j k} \varphi^{i j k}=0,
$$


where $\mathfrak{g}$ is the Lie algebra of $\lambda^{a}$ vector fields. As a consequence of the first condition above, $\varphi$ is invariant under the action of $\lambda^{a}$, see (246). Using the principal bundle language to describe the geometry of $M$ and since $i_{a} \varphi=0$ as well, $\varphi$ is the pull-back of a 3-form on base space $B^{7}$ which again is denoted with $\varphi$. The fibre is either $\mathbb{R}^{1,2}$ or $S L(2, \mathbb{R})$ up to discrete identifications. Moreover as a consequence of the last condition in $(244), B^{7}$ has a $G_{2}$ structure compatible with a metric connection, $\hat{\tilde{\nabla}}$, with skew-symmetric torsion. The metric, torsion and $G_{2}$ fundamental form on $B^{7}$ are given by $d \tilde{s}^{2}$ in $(242), \tilde{H}$ and $\varphi$, respectively. This in particular this implies that

$$
\begin{aligned}
& \tilde{H}=-\frac{1}{6}\left(\tilde{d} \varphi, \star_{7} \varphi\right) \varphi+\star_{7} \tilde{d} \varphi-\star_{7}\left(\tilde{\theta}_{\varphi} \wedge \varphi\right), \\
& \tilde{d} \star_{7} \varphi=\tilde{\theta}_{\varphi} \wedge \star_{7} \varphi,
\end{aligned}
$$

where

$$
\tilde{\theta}_{\varphi}=-\frac{1}{3} \star_{7}\left(\star_{7} \tilde{d} \varphi \wedge \varphi\right),
$$

is the Lee form of $\varphi$. The first condition is the expression of the torsion of $B^{7}$, and so of $\tilde{H}$, in terms of the geometry while the second condition is required for a 7-dimensional manifold with a $G_{2}$-structure to admit a compatible connection with skew-symmetric torsion $[147,148]$, see also $[142,143]$. The second universal condition in $(251)$ is $2 d \Phi-\tilde{\theta}_{\varphi}=0$ and so $B^{7}$ is conformally balanced.

The expression for $\tilde{H}$ in (253) depends on whether $G$ is abelian or not, see Table 3. As can be seen from (252), if $G$ is abelian, then the first term in (253) for $\tilde{H}$ will vanish. The requirement that $\left(\tilde{d} \varphi, \star_{7} \varphi\right)=0$ becomes a condition on the geometry. On the other hand if $G=S L(2, \mathbb{R})$, then the same term becomes proportional to the volume form of $\operatorname{SL}(2, \mathbb{R})$.

The curvature of the fibration $\mathcal{F}$ is a $G_{2}$ instanton,

$$
\mathcal{F}_{i j}=\frac{1}{2} \star_{7} \varphi_{i j}{ }^{k l} \mathcal{F}_{k l},
$$

with gauge group either $\mathbb{R}^{1,2}$ or $\operatorname{SL}(2, \mathbb{R})$. A similar condition is satisfied by gauge field strength $F$ as a consequence of the gaugino KSE (250).

\subsection{6. $S U(3), N=4$}

The $\hat{\nabla}$-parallel forms are the 1 -forms $\lambda^{a}, a=0,1,2,3$ which span $\Xi$, a Hermitian form $\omega_{I} \in \Lambda^{2}(\mathcal{T})$ associated with an almost complex structure $I$ and $(3,0)$-form $\chi \in \Lambda^{3}(\mathcal{T})$. Both $\omega_{I}$ and $\chi$ are the fundamental forms of SU(3) and satisfy the normalization conditions $\frac{1}{3 !} \wedge^{3} \omega_{I}=-i 2^{-3} \chi \wedge \bar{\chi}=d \operatorname{vol}_{\mathcal{T}}$.

The dilatino KSE implies the additional conditions

$$
\begin{aligned}
& \frac{1}{3 !} \epsilon^{a b c d} H_{b c d}-\frac{1}{2} \mathcal{F}_{i j}^{a} \omega_{I}^{i j}=0, \quad \mathcal{F}_{k l}^{a} I^{k}{ }_{i} I_{j}=\mathcal{F}_{i j}^{a}, \\
& \tilde{\mathcal{N}}(I)=0 .
\end{aligned}
$$

From the second condition, $\mathcal{F}^{a}$ is a $(2,0)$ and $(2,0)$ form with respect to $I$. In turn this gives that $\mathcal{L}_{a} \omega_{I}=0$. As $i_{a} \omega_{I}=0$, the Hermitian form $\omega_{I}$ is the pull-back of a Hermitian form on the base space $B^{6}$ which again we denote by $\omega_{I}$. Furthermore the last condition in (256) implies that $I$ is integrable. As a result $B^{6}$ is a Kähler manifold with torsion and so

$$
\tilde{H}=-i_{I} \tilde{d} \omega_{I}=\star_{6} \tilde{d} \omega_{I}-\star_{6}\left(\tilde{\theta}_{\omega_{I}} \wedge \omega_{I}\right) .
$$

The last condition in (244) that arises from the gravitino KSE on $M$ implies an additional geometric condition as some components of $\tilde{H}$ can be expressed in terms on both $\omega_{I}$ and $\chi$. This is

$$
\tilde{\theta}_{\omega}=\tilde{\theta}_{\operatorname{Rex}},
$$

where

$$
\tilde{\theta}_{\omega}=-\star_{6}\left(\star_{6} d \omega_{I} \wedge \omega_{I}\right), \quad \tilde{\theta}_{\operatorname{Re} \chi}=-\frac{1}{2} \star_{6}\left(\star_{6} d \operatorname{Re} \chi \wedge \operatorname{Re} \chi\right),
$$

are the Lee forms of $\omega_{I}$ and $\chi$ on $M$, respectively.

To make further progress on the geometry of $B^{6}$, suppose that $G$ is abelian. The first two conditions in (256) imply that $\mathcal{F} \in \Lambda_{\mathfrak{s u}(3)}^{2} \otimes \mathfrak{g}$ and $\chi$ is invariant under the action of $G$ as a consequence of (246). As $i_{a} \chi=0, \chi$ is the pull-back of a $(3,0)$-form on $B^{6}$. The base space, $B^{6}$, is a manifold with an $S U(3)$ structure compatible with a metric connection, $\hat{\tilde{\nabla}}$. The metric, skew-symmetric torsion $\tilde{H}$, and fundamental forms are given by $d \tilde{s}^{2}$ in (242), $\tilde{H}$ in (257), and $\omega_{I}$ and $\chi$, respectively. The condition (258) is that required for hol $(\hat{\tilde{\nabla}}) \subseteq S U(3)$. The second universal condition in (251) implies that $2 d \Phi-\tilde{\theta}_{\omega}=0$ and thus $B^{6}$ is conformally balanced with respect to $\omega_{I}$.

Next suppose that $G$ is non-abelian and therefore is either $\mathbb{R} \times S U(2)$ or $S L(2, \mathbb{R}) \times U(1)$ up to discrete identifications, see Table 3. It is clear from the first condition in (256) that $\mathcal{F} \in \Lambda_{\mathfrak{s u}(3) \oplus \mathbb{R}}^{2} \otimes \mathfrak{g}$ and so $\chi$ is not invariant under the $\mathbb{R}$ and $U(1)$ group actions, respectively. As a result, the canonical bundle of $B^{6}$ is twisted and therefore $B^{6}$ does not have an $S U(3)$ structure but 
rather a $U(3)$ one. Hence, $B^{6}$ is a manifold with $U(3)$ structure compatible with a connection with skew-symmetric torsion $\hat{\tilde{\nabla}}$, i.e. $B^{6}$ is a Kähler manifold with torsion. The metric, torsion and fundamental form are given by $d \tilde{s}^{2}$ in $(242), \tilde{H}$ in (257) and $\omega_{I}$, respectively. Moreover $B^{6}$ is conformally balanced with respect to $\omega_{I}$ as a consequence of the universal conditions in (251).

The gaugino KSE implies that the curvature of the gauge connection $F$ satisfies $F \in \Lambda_{\mathfrak{s u}(3)}^{2} \otimes \mathfrak{h}$. So $F$ as a 2-form in $\Lambda^{2}(\mathcal{T})$ is $(1,1)$ with respect to the complex structure $I$ and $\omega_{I}$-traceless, $\omega_{I}^{i j} F_{i j}=0$. This is the standard instanton condition on complex manifolds with an $S U(3)$ structure.

\subsection{7. $\operatorname{SU}(2), N=8$}

The $\hat{\nabla}$-parallel forms are the 1 -forms $\lambda^{a}, a=0, \ldots, 5$, which span $\Xi$ and the Hermitian forms $\omega_{r} \in \Lambda^{2}(\mathcal{T}), r=1,2,3$, associated to the (almost) complex structures, $I_{r}$, on $\mathcal{T}$ such $I_{3}=I_{1} I_{2}$ and $I_{1} I_{2}=-I_{2} I_{1}$.

In addition to the universal conditions (251), the dilatino KSE implies

$$
\begin{aligned}
& H_{a_{1} a_{2} a_{3}}+\frac{1}{3 !} \epsilon_{a_{1} a_{2} a_{3}}^{{ }^{{ }_{1} b_{2} b_{3}} H_{b_{1} b_{2} b_{3}}=0,} \\
& \mathcal{N}\left(I_{r}\right)=0, \quad \mathcal{F}^{a} \in \Lambda_{\mathfrak{s u}(2)}^{2} \otimes \mathfrak{g} .
\end{aligned}
$$

As $i_{a} \omega_{r}=0$, the last condition above implies that $\omega_{r}$ are the pull-backs of 2-forms on the base space $B^{4}$ which again we denote with $\omega_{r}$. The metric $d \tilde{s}^{2}$ is also the pull-back of a metric on $B^{4}$. This together with $\omega_{r}$ implies that $B^{4}$ admits three almost complex structures again denoted by $I_{r}$. These are integrable as a consequence of the second condition in (260). In fact $B^{4}$ has an $S U(2)$ structure compatible with a connection, $\hat{\tilde{\nabla}}$, with skew-symmetric torsion and thus $B^{4}$ is an HKT manifold. The metric, torsion and fundamental forms are $d \tilde{s}^{2}, \tilde{H}$ and $\omega_{r}$, respectively. The last equation in (244) on $\omega_{r}$ gives

$$
\tilde{H}=-i_{I_{r}} \tilde{d} \omega_{r}, \text { no summation over } r \text {. }
$$

The second universal condition in (251) implies that $2 d \Phi-\tilde{\theta}_{\omega_{r}}=0$, where $\tilde{\theta}_{\omega_{r}}$ is the Lee form of $\omega_{r}$. So $B^{4}$ is a conformally balanced HKT manifold. All conformally balanced 4-dimensional HKT manifolds are conformal to hyper-Kähler ones. So all the above conditions on $B^{4}$ can be solved to find that

$$
d \tilde{s}^{2}=e^{2 \Phi} d \tilde{s}_{\mathrm{hk}}^{2}, \quad \tilde{H}=-\star_{\mathrm{hk}} \tilde{d} e^{2 \Phi},
$$

where $d \tilde{s}_{\text {hk }}^{2}$ is a hyper-Kähler metric on $B^{4}$.

The first condition in (260) implies that the structure constants of the Lie algebra of 1-form bilinears are anti-self-dual. These are given in Table 3. Therefore up to discrete identifications the fibre Lie groups are

$$
\mathbb{R}^{5,1}, \quad S L(2, \mathbb{R}) \times S U(2), \quad C W_{6},
$$

where the radii of $S L(2, \mathbb{R})$ and $S U(2)$ are equal and the structure constants $\beta$ of $C W_{6}$ obey a self-duality condition, see Appendix C.2.

The last condition in (260) implies that the curvature of the principal bundle connection $\lambda^{a}$ is an anti-self-dual instanton on $B^{4}$ with gauge group one of those in (263). The gaugino KSE also implies that $F \in \Lambda^{2}(\mathcal{T}) \otimes \mathfrak{h}$ is an anti-self-dual instanton as well. This completes the description of geometry for these backgrounds

\subsection{Including $\alpha^{\prime}$ corrections}

Before we proceed to investigate some of the solutions of the theory, let us make some remarks regarding the geometry of solutions after taking into account the $\alpha^{\prime}$ corrections up to and including two loops in sigma model perturbation theory.

To begin suppose that the Bianchi identity of $H$ is modified by the anomaly as in (200), and the field equations and KSEs are appropriately modified as (203) and (201), respectively. First let us clarify the role of the spacetime curvature $\tilde{R}$ which enters in the expression for the anomaly and the field equations. It turns out that $\tilde{R}$ must satisfy the gaugino KSE in order for the anomalous Bianchi identity of $H$ and the KSEs to be compatible with the field equations. To see this, in the presence of anomalous contributions to the Bianchi identity of $H$ and the two loop corrections to the field equations, one can show using

$$
\hat{R}_{A[B, C D]}=-\frac{1}{3} \hat{\nabla}_{A} H_{B C D}-\frac{1}{6} d H_{A B C D},
$$

that (212) is modified as

$$
\begin{gathered}
\hat{R}_{A B, C D} \Gamma^{B} \Gamma^{C D} \epsilon=\frac{1}{36} \hat{\nabla}_{A}\left(\Gamma^{B} \partial_{A} \Phi-\frac{1}{12} H_{B C D} \Gamma^{B C D}\right) \epsilon \\
-\frac{\alpha^{\prime}}{4}\left(\tilde{R}_{A B, E F} \tilde{R}_{C D}{ }^{E F}-F_{A B a b} F_{C D}{ }^{a b}\right) \Gamma^{B} \Gamma^{C D} \epsilon+\mathcal{O}\left(\alpha^{\prime 2}\right) .
\end{gathered}
$$


If all the KSEs (201) are satisfied, and thus $\epsilon$ is a Killing spinor, then all the terms will vanish apart from that containing $\tilde{R}$. This is a restriction of the choice of $\tilde{R}$. A solution is to choose the spacetime connection $\tilde{R}$ to satisfy the same condition as that of the gaugino KSE on $F$.

In sigma model perturbation theory that will be described below, one can choose to this order $\tilde{R}=\check{R}$, where $\check{R}$ is the curvature of the connection with torsion $-H$ evaluated at zeroth order in $\alpha^{\prime}$. This is because

$$
\check{R}_{A B, C D}-\hat{R}_{C D, A B}=\frac{1}{2} d H_{A B C D}
$$

and $d H=0$ at zeroth order in $\alpha^{\prime}$. Then $\check{R}$ satisfies the gaugino KSE provided that all spinors that satisfy the gaugino KSE are $\hat{\nabla}$-parallel so that the holonomy of $\hat{\nabla}$ is contained in their isotropy group.

There are two points of view on how one should proceed from here. In the sigma model perturbation approach, one begins at zeroth order in $\alpha^{\prime}$ with $H$ closed, $d H^{(0)}=0$. Then this gets corrected order by order in perturbation theory as has been indicated. From this point of view, the anomaly correction is viewed as a first order correction and so on. In such a case, the geometry of the supersymmetric heterotic backgrounds at zeroth order is that we have already described in the previous sections. This of course will be corrected order by order in perturbation theory but as all such corrections are not known, it is not clear what the final outcome will be. However we do know that it is corrected to all orders in $\alpha^{\prime}[149,150]$.

An alternative point of view is to consider the anomalous Bianchi identity, the KSEs and field equations as exact at the order indicated in (201) and (203), respectively. The KSEs of the theory for $d H \neq 0$ have been solved in [126] and the geometry of the backgrounds has been identified. However the description of the descendants is rather more involved. Examples of Kähler and hyper-Kähler geometries with torsion for which $d H \neq 0$ have been first considered in [151]. An existence theorem for solutions of the differential system given by the anomalous Bianchi identity (200) and the KSEs (201) on Hermitian manifolds with $\operatorname{hol}(\hat{\nabla}) \subseteq S U$ and $\tilde{R}$ the Chern connection has been demonstrated [152]. Solutions of this system on manifolds with other geometric structures have also been given in [153]

The addition of higher curvature corrections up to and including the two loop order in sigma model perturbation theory does not alter the relation between the KSEs (201) and field equations (203) we have established for backgrounds with $d H=0$ in Sections 7.3.9 and 7.4.4. In particular if $\tilde{R}$ is chosen to satisfy the gaugino KSE, then for compact holonomy groups the KSEs imply all the field equations provided that the (anomalous) Bianchi identities are satisfied. For noncompact holonomy groups, the field equations given in (236) must also be satisfied. This follows from an investigation of the integrability conditions of the KSEs in (205) and after taking into account the anomalous Bianchi identity of $H$ and the two loop correction to the field equations.

\subsection{A brief overview of solutions}

All supersymmetric solutions of the heterotic theory that have been found can be organized according to the classification of the solutions to the KSEs we have presented. The purpose here is not to describe all solutions but rather to explain in which class some of the most well known solutions belong.

\subsubsection{WZW models}

Apart from the Minkowski vacuum, group manifold solutions to heterotic theory belong to descendants of backgrounds for which $\operatorname{hol}(\hat{\nabla})=\{1\}$. As we have not describe the geometry of descendants, we shall give a brief description of the descendants of $K=\{1\}$. The backgrounds are group manifolds which are parallelizable with a connection with skewsymmetric torsion. Therefore they admit 16 parallel spinors. If all parallel spinors also solve the gaugino KSE, then $F=0$.

It remains to solve the dilatino KSE. For this there are two cases to consider depending on whether or not the 1 -form $d \Phi$ is null. Suppose that $d \Phi$ is not null and $|d \Phi|^{2} \neq 0$. In this case, one can show that the dilatino Killing spinor equation $[154,155]$ implies that

$$
\Pi=\frac{1}{2}+\frac{\partial_{M} \Phi H_{N P Q} \Gamma^{M N P Q}}{24|d \Phi|^{2}},
$$

is a projector, $\Pi^{2}=\Pi$. Since $\operatorname{tr} \Pi=8$, backgrounds with $d H=\hat{R}=0$ and $|d \Phi| \neq 0$ preserve half of the supersymmetry. Moreover one can also show that $d \Phi$ is $\nabla$-parallel, spacelike and $i_{d \Phi} H=0$, see e.g. $[115,154]$. Thus the spacetime up to discrete identifications is a product $M=N \times \mathbb{R}$, where $\mathbb{R}$ is spanned by $d \Phi$. Using this and the classification of Lorentzian Lie groups up to dimension 9, one finds that up to local isometries the spacetime is one of the following groups

$$
\begin{aligned}
& S L(2, \mathbb{R}) \times S U(2) \times S U(2) \times \mathbb{R}, \quad S L(2, \mathbb{R}) \times S U(2) \times \mathbb{R}^{4}, \\
& \mathbb{R}^{1,1} \times S U(3), \quad \mathbb{R}^{3,1} \times S U(2) \times S U(2), \\
& \mathbb{R}^{6,1} \times S U(2), \quad C W_{4} \times S U(2) \times \mathbb{R}^{3}, \quad C W_{6} \times S U(2) \times \mathbb{R},
\end{aligned}
$$

where $C W_{n}$ are the Cahen-Wallach group manifolds described in Appendix C.2. Moreover $H$ is determined from the structure constants of the group manifolds and all these are linear dilaton backgrounds.

On the other hand if $(d \Phi)^{2}=0$, i.e. either $d \Phi$ is null or $d \Phi=0$, then $H$ is null. The condition $i_{d \Phi} H=0$, implies that these backgrounds preserve at least eight supersymmetries. Such solutions are locally isometric to $C W_{n} \times \mathbb{R}^{10-n}$ for $n=2,4,6$. The 
dilaton is linear if $d \Phi \neq 0$ otherwise it is constant, and $H$ is determined by the structure constants of $C W$ spaces. The group manifolds $C W_{10}$ are also solutions and for generic structure constants $\beta \in \Lambda^{2}\left(\mathbb{R}^{8}\right)$ preserve 8 supersymmetries. Moreover for some special choice of $\beta$, these manifolds exhibit supersymmetry enhancement to 10,12 and 14 supersymmetries.

\subsection{2. $N=8$ solutions with $S U(2)$ and $\mathbb{R}^{8}$ holonomy}

The half-supersymmetric solutions of heterotic theory including $\alpha^{\prime}$ corrections have been investigated in [145]. Here we shall take $d H=0$ and first consider the $S U(2)$ holonomy backgrounds. From the results in Section 7.4.7 the spacetime metric and $H$ can be written as

$$
\begin{aligned}
d s^{2} & =\eta_{a b} \lambda^{a} \lambda^{b}+e^{2 \Phi} d s_{\mathrm{hk}}^{2}, \\
H & =\frac{1}{3} \eta_{a b} \lambda^{a} \wedge d \lambda^{b}+\frac{2}{3} \eta_{a b} \lambda^{a} \wedge \mathcal{F}^{b}-\star_{\mathrm{hk}} \tilde{d} e^{2 \Phi},
\end{aligned}
$$

where $d s_{\mathrm{hk}}^{2}$ is a $d=4$ hyper-Kähler metric and $\lambda$ is an anti-self-dual principal bundle connection with gauge group $\mathbb{R}^{5,1}$, $S L(2, \mathbb{R}) \times S U(2)$ or $C W_{6}$ with self-dual structure constants.

To find explicit examples, one has to specify a $d=4$ hyper-Kähler manifold, an anti-self dual instanton connection over it and to determine the dilaton. The latter is found by exploring the Bianchi identity (200) of $H$, i.e. $d H=0$. This gives

$$
-\nabla_{\mathrm{hk}}^{2} e^{2 \Phi}-\frac{1}{2} \eta_{a b} \mathcal{F}_{i j}^{a} \mathcal{F}^{b i j}=0 .
$$

There are many solutions that can be constructed using the above data. These include the 5-brane solution of [156] which is given by setting $B^{4}=\mathbb{R}^{4}$ with the Euclidean metric and $G=\mathbb{R}^{5,1}$. In such a case, one has

$$
\begin{aligned}
d s^{2} & =d s^{2}\left(\mathbb{R}^{5,1}\right)+e^{2 \Phi} d s^{2}\left(\mathbb{R}^{4}\right), \quad H=-\star d h, \\
e^{2 \Phi} & =1+\frac{Q}{|x|^{2}},
\end{aligned}
$$

where $Q$ is related to the charge of the brane. More general classes of solutions have been constructed in [145]. The $S U(2)$ holonomy class of solutions also includes the Kaluza-Klein monopole for which $B^{4}$ is equipped with the Gibbons-Hawking metric, the dilaton is constant and $H=0$.

Next consider the holonomy the $\mathbb{R}^{8}$ solutions. In this case, there is a choice of coordinates $\left(u, v, x^{i}\right)$ such that

$$
\begin{aligned}
& d s^{2}=2 \mathbf{e}^{-} \mathbf{e}^{+}+d s^{2}\left(\mathbb{R}^{8}\right), \quad H=d\left(\mathbf{e}^{-} \wedge \mathbf{e}^{+}\right), \\
& \mathbf{e}^{-}=h^{-1} d v, \quad \mathbf{e}^{+}=d u+V d v+n_{i} d x^{i} .
\end{aligned}
$$

All components of the metric, $\Phi$ and $H$ depend on $v$ and $x$, and $X=\partial_{u}$ is the null parallel vector field.

The solutions of the Killing spinor equations are determined up to the functions $h$ and $V$, and the 1-form $n$. These in turn can be found by solving the field equations (236). In addition if one assumes that $h, V$ and $n$ are $v$ independent, then the field equations imply that

$$
\partial_{i}^{2} h=\partial_{i}^{2} V=0, \quad \partial^{i} d n_{i j}=0,
$$

and $e^{2 \Phi}=h^{-1}$. So $h$ and $V$ are harmonic functions of $\mathbb{R}^{8}$ and $d n$ satisfies the Maxwell equations on $\mathbb{R}^{8}$. The solution is a superposition of fundamental strings [157], pp-waves and null rotations. The NS5-brane solution, the fundamental string, the pp-wave and the Kaluza-Klein monopole are considered the elementary branes of heterotic theory. In perturbation theory, the string solution is considered as the back-reaction of the elementary string of theory while the 5-brane is its magnetic dual and it is solitonic.

\subsubsection{Compactification vacua}

Minkowski space compactification vacua are warped product solutions $\mathbb{R}^{n-1,1} \times{ }_{w} N^{d-n}$ with fields which are invariant under the Poincare symmetry of $\mathbb{R}^{n-1,1}$ and $N^{10-n}$ restricted to be a compact manifold without boundary. In supergravity theories, it is known that there are no such smooth solutions, $n>2$, with non-vanishing fluxes [158,159], see also Section 12.5. In heterotic theory this non-existence theorem applies to the zeroth order in $\alpha^{\prime}$ sector of the theory. To see this suppose that the warp factor is constant and that $H$ does not have a non-vanishing component along $\mathbb{R}^{n-1,1}$. In such a case, the theorem follows from the dilaton field equation in (203) upon an application of the Hopf maximum principle. Indeed as $N^{10-n}$ is compact without boundary, $\Phi$ has an absolute maximum and an absolute minimum which are critical points and so its hessian is negative or positive definite, respectively. However the $H^{2}$ term in the dilaton field equation without the two-loop contribution is positive definite which is a contradiction unless $H=0$ and $\Phi$ is constant. Thus no smooth solutions exist with non-trivial fluxes.

The same conclusion holds whenever $H$ has also non-vanishing components along $\mathbb{R}^{n-1,1}$ and there is a non-trivial warp factor. The theorem again follows upon application of the Hopf maximum principle on both the warp factor and the dilaton field equations. Again as can be seen from the dilaton field equation in (203) higher order corrections can modify this conclusion as the terms proportional to $\alpha^{\prime}$ may not have a definite sign, see also $[138,160]$. 
Suppose that $H=0$, the dilaton constant and let $N^{10-n}$ be a compact manifold without boundary. In such case, $N^{10-n}$ up to discrete identifications is a product of the appropriate dimension of the Berger type of manifolds $N^{8}$ with holonomy $\operatorname{Spin}(7), N^{7}$ with holonomy $G_{2}$, Calabi-Yau $C Y_{8}$ and $C Y_{6}$ with holonomy $S U(4)$ and $S U(3)$, respectively, $K_{3}$ with holonomy $S U(2)$ and tori $T^{k}$ with holonomy $\{1\}$.

Amongst these, the compactifications on $N^{8}$ with holonomy $\operatorname{Spin}(7), S U(4)$ and $S U(2) \times S U(2)$ to two dimensions belong to the class of heterotic solutions for which the holonomy of the connection with torsion, hol $(\hat{\nabla})$, is in one of the noncompact groups $\operatorname{Spin}(7) \ltimes \mathbb{R}^{8}, S U(4) \ltimes \mathbb{R}^{8}$ and $(S U(2) \times S U(2)) \ltimes \mathbb{R}^{8}$, respectively, see Table 1 . The two dimensional theories have chiral supersymmetry. The compactification on $G_{2}$ manifolds to three dimensions belongs to the heterotic solutions for which $\operatorname{hol}(\hat{\nabla})=G_{2}$ and has been investigated in $[161,162]$. Similarly the compactification of heterotic theory on $C Y_{6}$ belongs to the holonomy $\operatorname{hol}(\hat{\nabla})=S U(3)$ class of solutions of the heterotic theory. This leads to an $\mathcal{N}=1$ theory in four dimensions and as a result it has been extensively investigated from the phenomenological point of view [163]. It is also instrumental in the understanding of mirror symmetry, see e.g. [164-166]. The $K_{3}$ and $T^{k}$ compactifications belong to the holonomy hol $(\hat{\nabla})=S U(2)$ and $\operatorname{hol}(\hat{\nabla})=\{1\}$ solutions of the heterotic theory, respectively, and they have found applications in the understanding of string dualities.

\subsubsection{Non-existence theorem for euclidean signature solutions}

The KSEs (201) and field equations (203) of heterotic supergravity can also be considered on Euclidean signature manifolds in all dimensions. Of course the representation of the supersymmetry parameter $\epsilon$ changes with dimension. The parallel spinors have always compact isotropy groups and therefore may not admit non-vanishing 1-form bilinears. Nevertheless some of the results we have described carry over to the Euclidean case. In particular, the relation between the KSEs and field equations remains unchanged. A consequence of this is that the KSEs imply the field equations provided the (anomalous) Bianchi identity (200) of $H$ is still valid.

As the KSEs imply the field equations, and in the absence of $\alpha^{\prime}$ corrections, one concludes that $H=0$ and $\Phi=$ const. This follows from an application of the Hopf maximum principle on $\Phi$ and the dilaton field equation. Therefore at zeroth order in $\alpha^{\prime}$, there are no smooth Euclidean signature solutions which solve the KSEs of heterotic theory with non-trivial fluxes. For holonomy $S U(n)$ solutions this had already been shown by the authors of $[138,160]$ using a complex geometry argument. In particular they demonstrated the non-existence of a certain holomorphic section in the canonical bundle for such manifolds and also extended the result under some conditions to include $\alpha^{\prime}$ corrections. Therefore the existence of smooth Euclidean signature compact solutions requires that $\alpha^{\prime}$ corrections are included and in particular $d H \neq 0$.

\subsubsection{Brane superpositions, black holes and AdS/CFT}

Another class of solutions which has widespread applications is that of superpositions of the fundamental branes of heterotic string theory. Such a solution is the fundamental string within a 5-brane with a pp-wave propagating along the string. The solution depends on 3-harmonic functions on the hyper-Kähler manifold $B^{4}, h_{1}, h_{5}$ and $h_{w}$, which are related to the string, 5-brane and pp-wave solitons, respectively, and reads

$$
\begin{aligned}
d s^{2} & =h_{1}^{-1}\left(2 d v\left(d u-\frac{1}{2}\left(h_{w}-1\right) d v\right)\right)+d s^{2}\left(\mathbb{R}^{4}\right)+h_{5} d s_{\mathrm{hk}}^{2}\left(B^{4}\right), \\
H & =d v \wedge d u \wedge d h_{1}-\star_{\mathrm{hk}} d h_{5}, \quad e^{2 \Phi}=h_{1}^{-1} h_{5} .
\end{aligned}
$$

Setting $B^{4}=\mathbb{R}^{4}$, the solution can be written down explicitly [167] and gives upon reduction a black hole solution in 5-dimensions. This has been extensively investigated in [168] as the entropy of this black hole can be computed using a microscopic argument in IIB string theory as in [169].

Another widely investigated solution for microscopic entropy computations is that for which $B^{4}$ is the Gibbons-Hawking manifold and $h_{1}, h_{5}$ and $h_{w}$ are invariant under the tri-holomorphic isometry of $B^{4}$. This solution gives rise to a 4-dimensional black hole. For more recent investigations of this system that include higher order corrections see [170]. All these solutions are examples of $N=4$ backgrounds with hol $(\hat{\nabla}) \subseteq(S U(2) \times S U(2)) \ltimes \mathbb{R}^{8}$, see also [171] for more examples of solutions for which hol $(\hat{\nabla})$ is a non-trivial group.

Amongst the heterotic solutions are also backgrounds which have been considered as gravitational duals of gauge theories in the context of the AdS/CFT correspondence. One such solution is that of [172] which has been proposed as the gravitational dual of minimal $\mathcal{N}=1, d=4$ supersymmetric gauge theory in [173]. This is a solution of heterotic supergravity which preserves $N=4$ supersymmetries. It is an example of a heterotic solution with $\operatorname{hol}(\hat{\nabla}) \subseteq S U(3), G=\mathbb{R}^{3,1}$ and $d H=0$. This has been shown in [139], where the $\hat{\nabla}$-parallel forms are also explicitly given. It evades the non-existence theorem of [138] explained above because it is not compact.

\section{Geometry of $d=11$ supergravity backgrounds}

The $d=11$ supergravity [174] has been proposed as the effective theory of M-theory [1] which in turn arises as a strong coupling limit of IIA strings [106]. The supersymmetric solutions of $d=11$ supergravity include the M2and M5-branes [175,176], which are thought of as the "elementary" solitons of M-theory, and their superpositions and intersections [177-179]. These have extensively been used to give evidence in favour of the existence of M-theory and 
of string dualities. More recently they have found a key role in the AdS/CFT correspondence. There are some extensive reviews on the supersymmetric solutions of $d=11$ supergravity theory, see e.g. [6,180]. Here we shall be mostly concerned with the systematics of solving the KSE of $d=11$ supergravity. Later we shall explore some applications in the theory of compactifications and the AdS/CFT correspondence. The bosonic fields of the theory are a metric and a 4-form field strength $F, d F=0$. The action, KSE and a summary of other properties of the theory that are used in this review can be found in Appendix E.1.

\subsection{Spinors and the KSE}

The theory has a single fermionic field, a gravitino, whose supersymmetry variation gives the KSE

$$
\mathcal{D}_{M} \epsilon=0,
$$

where

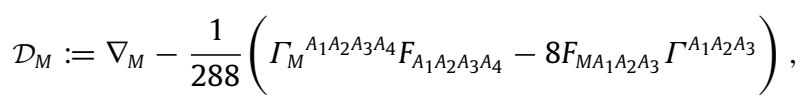

is the supercovariant derivative, $\nabla$ is the Levi-Civita connection of the spacetime and the supersymmetry parameter, $\epsilon$, is in the 32-dimensional Majorana representation, $\Delta_{\mathbf{3 2}}$, of $\operatorname{Spin}(10,1)$.

In anticipation of using the spinorial geometry method to solve (275), let us first realize $\Delta_{\mathbf{3 2}}$ in terms of forms. First, one can begin with the Dirac representation of $\operatorname{Spin}(10)$. This is identified with $\Lambda^{*}\left(\mathbb{C}^{5}\right)$ as has already been described in Appendix B. This extends to a real presentation of $\operatorname{Pin}(10)$ and so to a real representation of $\operatorname{Spin}(10,1)$. In particular, the gamma matrix along the time direction is $\Gamma_{0}:=\Gamma_{1} \ldots \Gamma_{\natural}$, where $\Gamma_{\natural}:=\Gamma_{10}$. The reality condition is imposed using the anti-linear map $r_{B}=\Gamma_{0} b *$. Therefore a basis in $\Delta_{32}$ is given by the forms

$$
e_{a_{1} \cdots a_{k}}+(-1)^{[k / 2]} \star e_{a_{1} \cdots a_{k}}, \quad i e_{a_{1} \cdots a_{k}}-i(-1)^{[k / 2]} \star e_{a_{1} \cdots a_{k}},
$$

where $a_{1}<\cdots<a_{k}, a_{1}, a_{2}, \ldots, a_{5}=1, \ldots, 5, k=0,1,2$, and star is the Hodge duality operation in $\Lambda^{*}\left(\mathbb{C}^{5}\right)$.

Alternatively, one can begin with the Dirac representation of $\operatorname{Spin}(9,1)$ described in Appendix B and then construct $\Delta_{32}$ by setting the gamma matrix along the 10th direction to $\Gamma_{\mathrm{\natural}}=-\Gamma_{0123456789}$. Moreover, the reality condition is imposed using the anti-linear map $r_{B}=-\Gamma_{0} b *$. Therefore a basis in $\Delta_{32}$ can be chosen as

$$
\begin{aligned}
& e_{a_{1} \cdots a_{k}}+(-1)^{[k / 2]+k} \star e_{a_{1} \cdots a_{k}}, \quad i e_{a_{1} \cdots a_{k}}-i(-1)^{[k / 2]+k} \star e_{a_{1} \cdots a_{k}}, \\
& e_{a_{1} \cdots a_{k} 5}+(-1)^{[k / 2]+k} \star e_{a_{1} \cdots a_{k} 5}, \quad i e_{a_{1} \cdots a_{k} 5}-i(-1)^{[k / 2]+k} \star e_{a_{1} \cdots a_{k} 5},
\end{aligned}
$$

where $a_{1}<\cdots<a_{k}$, but now $a_{1}, \ldots, a_{4}=1,2,3,4, k=0,1,2$, and star is the Hodge duality operation in $\Lambda^{*}\left(\mathbb{C}^{4}\right)$.

There are two types of orbits of $\operatorname{Spin}(10,1)$ on $\Delta_{32}$. One has isotropy group $S U(5)$ and the other has isotropy group $\operatorname{Spin}(7) \ltimes \mathbb{R}^{9}=\left(\operatorname{Spin}(7) \ltimes \mathbb{R}^{8}\right) \times \mathbb{R},[181,182]$. The former is an orbit of co-dimension 1 . Representatives of the two orbits can be chosen as

$$
1+e_{12345} \text { and } 1+e_{1234},
$$

written in the bases (277) and (278), respectively.

\section{2. $N=1 S U(5)$ backgrounds}

There are two types of $N=1$ supersymmetric $d=11$ supergravity backgrounds depending on whether the Killing spinor has isotropy group $\operatorname{SU}(5)$ or $\operatorname{Spin}(7) \ltimes \mathbb{R}^{9}$ in $\operatorname{Spin}(10,1)$. The KSE has been solved using the bilinears method in $[183,184]$. Here we shall present details of the solution of the KSE for the SU(5) invariant Killing spinor in the spinorial geometry method $[9,185]$ as the proof is shorter than when employing the bilinears method and moreover it can be adapted to classify the solutions that preserve a near maximal number of supersymmetries. We shall also outline the geometry of backgrounds admitting a $\operatorname{Spin}(7) \ltimes \mathbb{R}^{9}$ invariant Killing spinor.

\subsubsection{The solution of the linear system}

We begin by choosing the Killing spinor as

$$
\epsilon=f\left(1+e_{12345}\right),
$$

where $f$ is a (local) spacetime function. This function appears because the orbits with isotopy group SU(5) are of co-dimension 1 in $\Delta_{32}$ and they are not isolated. Adapting on the spacetime the spinorial geometry coframe $\left\{\mathbf{e}^{0}, \mathbf{e}^{i}: i=1, \ldots, \downarrow\right\}=$ $\left\{\mathbf{e}^{0}, \mathbf{e}^{\alpha}, \mathbf{e}^{\bar{\alpha}}: \alpha=1, \ldots, 5\right\}$, we decompose the metric and fluxes as

$$
\begin{aligned}
& d s^{2}=-\left(\mathbf{e}^{0}\right)^{2}+\delta_{i j} \mathbf{e}^{i} \mathbf{e}^{j}, \\
& F=\frac{1}{3 !} G_{i j k} \mathbf{e}^{0} \wedge \mathbf{e}^{i} \wedge \mathbf{e}^{j} \wedge \mathbf{e}^{k}+\frac{1}{4 !} \tilde{F}_{i j k l} \mathbf{e}^{i} \wedge \mathbf{e}^{j} \wedge \mathbf{e}^{k} \wedge \mathbf{e}^{l} .
\end{aligned}
$$


Note that $d s^{2}=-\left(\mathbf{e}^{0}\right)^{2}+2 \delta_{\alpha \bar{\beta}} \mathbf{e}^{\alpha} \mathbf{e}^{\bar{\beta}}$ and the fluxes $G$ and $\tilde{F}$ can be decomposed in a similar way. Substituting (280) into the Killing spinor equation (275), one derives a linear system which reads

$$
\begin{aligned}
& \partial_{0} \log f+\frac{1}{2} \Omega_{0, \alpha \bar{\beta}} g^{\alpha \bar{\beta}}-\frac{i}{24} \tilde{F}_{\alpha}{ }^{\alpha}{ }^{\beta}=0, \\
& i \Omega_{0,0 \bar{\alpha}}+\frac{1}{3} G_{\bar{\alpha} \beta}{ }^{\beta}+\frac{i}{72} \tilde{F}_{\beta_{1} \beta_{2} \beta_{3} \beta_{4}} \chi^{\beta_{1} \beta_{2} \beta_{3} \beta_{4}}=0, \\
& \Omega_{0, \bar{\alpha} \bar{\alpha}}-\frac{i}{6} \tilde{F}_{\bar{\alpha} \bar{\beta} \gamma}{ }^{\gamma}-\frac{1}{18} G_{\gamma_{1} \gamma_{2} \gamma_{3}} \chi^{\gamma_{1} \gamma_{2} \gamma_{3} \bar{\alpha} \bar{\beta}}=0,
\end{aligned}
$$

and

$$
\begin{aligned}
& \partial_{\bar{\alpha}} \log f+\frac{1}{2} \Omega_{\bar{\alpha}, \beta \bar{\gamma}} g^{\beta \bar{\gamma}}+\frac{i}{12} G_{\bar{\alpha} \gamma}^{\gamma}-\frac{1}{72} \chi_{\bar{\alpha}}^{\beta_{1} \beta_{2} \beta_{3} \beta_{4}} \tilde{F}_{\beta_{1} \beta_{2} \beta_{3} \beta_{4}}=0, \\
& \partial_{\bar{\alpha}} \log f-\frac{1}{2} \Omega_{\bar{\alpha}, \beta \bar{\gamma}} g^{\beta \bar{\gamma}}+\frac{i}{4} G_{\bar{\alpha} \gamma}{ }^{\gamma}=0, \\
& i \Omega_{\bar{\alpha}, 0 \bar{\beta}}+\frac{1}{6} \tilde{F}_{\bar{\alpha} \bar{\beta} \gamma}{ }^{\gamma}-\frac{i}{18} \chi_{\bar{\alpha} \bar{\beta}} \gamma_{1} \gamma_{2} \gamma_{3} G_{\gamma_{1} \gamma_{2} \gamma_{3}}=0, \\
& i \Omega_{\bar{\alpha}, 0 \beta}+\frac{1}{12} g_{\bar{\alpha} \beta} \tilde{F}_{\gamma} \gamma_{\delta}^{\delta}+\frac{1}{2} \tilde{F}_{\bar{\alpha} \beta \gamma}^{\gamma}=0, \\
& \Omega_{\bar{\alpha}, \bar{\beta} \bar{\gamma}}+\frac{i}{6} G_{\bar{\alpha} \bar{\beta} \bar{\gamma}}-\frac{1}{12} \chi_{\bar{\alpha} \bar{\beta} \bar{\gamma}} \gamma_{1} \gamma_{2} \tilde{F}_{\gamma_{1} \gamma_{2} \delta} \delta-\frac{1}{12} \tilde{F}_{\bar{\alpha} \gamma_{1} \gamma_{2} \gamma_{3}} \chi^{\gamma_{1} \gamma_{2} \gamma_{3} \bar{\beta} \bar{\gamma}}=0 \text {, } \\
& \left.\Omega_{\bar{\alpha}, \beta \gamma}-\frac{i}{2} G_{\bar{\alpha} \beta \gamma}-\frac{i}{3} g_{\bar{\alpha}[\beta} G_{\gamma}\right]_{\delta}^{\delta}-\frac{1}{36} \tilde{F}_{\bar{\alpha} \bar{\gamma}_{1} \bar{\gamma}_{2} \bar{\gamma}_{3}} \chi^{\bar{\gamma}_{1} \bar{\gamma}_{2} \bar{\gamma}_{3}}{ }_{\beta \gamma}=0 .
\end{aligned}
$$

where $\chi_{\alpha_{1} \alpha_{2} \alpha_{3} \alpha_{4} \alpha_{5}}=\sqrt{2} \epsilon_{\alpha_{1} \alpha_{2} \alpha_{3} \alpha_{4} \alpha_{5}}$,

The solution of the linear system above expresses some of the fluxes in terms of geometry as

$$
\begin{aligned}
& \tilde{F}_{\beta_{1} \ldots \beta_{4}}=\frac{1}{2}\left(-\Omega_{0,0 \bar{\alpha}}+2 \Omega_{\bar{\alpha}, \beta}{ }^{\beta}\right) \chi^{\bar{\alpha}} \beta_{1} \ldots \beta_{4}, \quad G_{\bar{\alpha} \beta}{ }^{\beta}=-2 i \Omega_{\bar{\alpha}, \beta} \beta-2 i \Omega_{0,0 \bar{\alpha}}, \\
& \tilde{F}_{\alpha}{ }^{\alpha}{ }^{\beta}=12 i \Omega_{\bar{\alpha}, 0 \beta} g^{\bar{\alpha} \beta}, \quad \tilde{F}_{\beta \bar{\alpha} \gamma}{ }^{\gamma}=2 i \Omega_{\bar{\alpha}, 0 \beta}+2 i g_{\bar{\alpha} \beta} \Omega_{\bar{\gamma}, 0 \delta} g^{\bar{\gamma} \delta}, \\
& G_{\bar{\alpha} \beta \gamma}=-2 i \Omega_{\bar{\alpha}, \beta \gamma}+2 i g_{\bar{\alpha}[\beta} \Omega_{0,0 \gamma]}, \quad G_{\bar{\alpha}_{1} \bar{\alpha}_{2} \bar{\alpha}_{3}}=6 i \Omega_{\left[\bar{\alpha}_{1}, \bar{\alpha}_{2} \bar{\alpha}_{3}\right]} \\
& \tilde{F}_{\bar{\alpha} \beta_{1} \beta_{2} \beta_{3}}=\frac{1}{2}\left[\Omega_{\bar{\alpha}, \bar{\gamma}_{1} \bar{\gamma}_{2}} \chi^{\bar{\gamma}_{1} \bar{\gamma}_{2}} \beta_{\beta_{1} \beta_{2} \beta_{3}}+3 \Omega_{\bar{\gamma}_{1}, \bar{\gamma}_{2} \bar{\gamma}_{3}} \chi^{\bar{\gamma}_{1} \bar{\gamma}_{2} \bar{\gamma}_{3}}{ }_{\left[\beta_{1} \beta_{2}\right.} g_{\left.\beta_{3}\right] \bar{\alpha}}\right. \\
& \left.+12 i \Omega_{\left[\beta_{1}, 0 \beta_{2}\right.} g_{\left.\beta_{3}\right] \bar{\alpha}}\right] \text {. }
\end{aligned}
$$

In addition, one finds the conditions on the geometry

$$
\begin{aligned}
& \partial_{0} \log f=0, \quad \Omega_{\bar{\alpha}, 0 \beta}+\Omega_{\beta, 0 \bar{\alpha}}=0, \quad 2 \partial_{\bar{\alpha}} \log f+\Omega_{0,0 \bar{\alpha}}=0, \\
& \Omega_{0, \bar{\alpha} \bar{\beta}}=\Omega_{\bar{\alpha}, 0 \bar{\beta}}, \quad \Omega_{0, \beta \bar{\alpha}} g^{\beta \bar{\alpha}}+\Omega_{\bar{\alpha}, 0, \beta} g^{\bar{\alpha} \beta}=0, \\
& \Omega_{\bar{\alpha}, \beta \gamma} g^{\bar{\alpha} \beta}-\Omega_{\gamma, \beta}{ }^{\beta}-\Omega_{0,0 \gamma}=0 .
\end{aligned}
$$

It is clear by construction that both the linear system and its solution are expressed in terms of the representations of the isotropy group $S U(5)$ of the Killing spinor. Furthermore, observe that not all components of the fluxes are expressed in terms of the geometry. In particular the Hermitian-traceless component of $\tilde{F}_{\alpha \bar{\beta} \gamma \bar{\delta}}$ is not determined in terms of geometry. This signals that the implementation of field equations and Bianchi identities will not be straightforward for solutions of the KSE with few supersymmetries.

\subsubsection{Geometry}

The spacetime admits a 1 -form, a 2 -form and a 5 -form bilinear of the Killing spinor $\epsilon=f\left(1+e_{12345}\right)$. These can be easily computed to find

$$
\begin{aligned}
X & =f^{2} \mathbf{e}^{0}, \quad \omega=-f^{2}\left(\mathbf{e}^{1} \wedge \mathbf{e}^{6}+\cdots+\mathbf{e}^{5} \wedge \mathbf{e}^{\natural}\right), \\
\tau & =f^{2} \operatorname{Im}\left[\left(e^{1}+i e^{6}\right) \wedge \cdots \wedge\left(e^{5}+i e^{\natural}\right)\right]+\frac{1}{2} f^{2} e^{0} \wedge \omega \wedge \omega .
\end{aligned}
$$

We shall use these to interpret the conditions we have found on the geometry of spacetime in (285).

First it is straightforward to verify that the first three conditions together with the symmetric part of the fourth condition in (285) imply that $X$ is a Killing vector field. Moreover upon using the Bianchi identity of $F$, one can also show that $\mathcal{L}_{X} F=0$ and therefore all the fields are invariant under the action of $X$. Furthermore, if one also uses in addition the antisymmetric part of the fourth condition and the fifth condition in (285), one can establish that $\epsilon$ is invariant under the action on $X$. Thus we have shown that

$$
\mathcal{L}_{X} g=\mathcal{L}_{X} F=0, \quad \mathcal{L}_{X} \epsilon=0,
$$


where $\mathcal{L}_{X}$ in the last condition above is the spinorial Lie derivative defined in (38). The last condition in (285) can be expressed as

$$
W_{5}+2 d f=0,
$$

where $\left(W_{5}\right)_{i}=\frac{1}{40} \chi^{j_{1} \ldots j_{5}} \nabla_{[i} \chi_{\left.j_{1} \ldots j_{5}\right]}$.

As has already been mentioned, the geometric conditions in (287) are universal and hold for the $N=1$ backgrounds of all supergravity theories. The last condition is required for the Killing superalgebras to close, see Section 8.6.

To locally describe the geometry of spacetime, notice that a consequence of the invariance of $\epsilon$ under the action of $X$ is that all form bilinears of the Killing spinor are also invariant. So we have

$$
\mathcal{L}_{X} \omega=\mathcal{L}_{X} \tau=0 .
$$

The spacetime can locally be described as a fibration whose fibres have a tangent space spanned by $X$ where the base space $B$ is a 10-dimensional space with an $S U(5)$ structure that satisfies (288). This follows because both $\omega$ and $\tau$ are invariant under $X$ and that $i_{X} \omega=i_{X} \tau=0$. Therefore both $\omega$ and $\tau$ "descend" as fundamental $S U(5)$ forms on the base space $B$. If one adapts a local coordinate $t$ along $X, X=\partial_{t}$, then $\mathbf{e}^{0}=f^{2}\left(d t+w_{i} \mathbf{e}^{i}\right)$.

The conditions relating the fluxes to the geometry, (284), can also be expressed in a real basis in terms of covariant and/or exterior derivatives of form bilinears (286). However, the final expressions are rather involved, see [9], and we shall not describe them here.

\subsection{Geometry of $\operatorname{Spin}(7) \ltimes \mathbb{R}^{9}$ backgrounds}

To describe the geometry of $d=11$ backgrounds admitting a Killing spinor which has isotropy group $\operatorname{Spin}(7) \ltimes \mathbb{R}^{9}$ in $\operatorname{Spin}(10,1)$, one can choose $\epsilon=1+e_{1234}$, where the spinor is written in the basis (278). The spacetime geometry is best described in a coframe with respect to which the metric can be written

$$
d s^{2}=2 \mathbf{e}^{-} \mathbf{e}^{+}+\left(\mathbf{e}^{9}\right)^{2}+\delta_{i j} \mathbf{e}^{i} \mathbf{e}^{j},
$$

for $i, j=1, \ldots, 8$. Note that this coframe is different from that used in [184] to give the solution of the KSE. This coframe is determined up to $\left(\operatorname{Spin}(7) \ltimes \mathbb{R}^{8}\right) \times \mathbb{R}$ gauge transformations which transform the coframe as that in (189).

For this class of solutions, the form bilinears are

$$
X=\mathbf{e}^{-}, \quad \tau=\mathbf{e}^{-} \wedge \phi,
$$

where

$$
\phi=\frac{1}{4 !} \phi_{i j k \ell} \mathbf{e}^{i} \wedge \mathbf{e}^{j} \wedge \mathbf{e}^{k} \wedge \mathbf{e}^{\ell},
$$

is the fundamental self-dual 4 -form of $\operatorname{Spin}(7)$.

In either the bilinears or spinorial geometry methods for solving the KSE, the solution yields the following conditions on the spacetime geometry

$$
\Omega_{(M, N)+}=0, \quad \Omega_{[i, j]+}^{7}=0, \quad \Omega_{i, 9+}=0, \quad \Omega_{+, 9 i}=0, \quad \Omega_{+, i j}^{7}=0,
$$

and

$$
\Omega_{-,+9}=-\frac{1}{4} \Omega^{i}{ }_{, i 9}, \quad \Omega_{9, i j}^{7}=-\Omega_{[i, j] 9}^{7},
$$

and

$$
\Omega_{9,9 i}-6 \Omega_{i,+-}=-\phi_{i}^{j_{1} j_{2} j_{3}} \Omega_{j_{1}, j_{2} j_{3}}-2 \Omega^{j}{ }_{j i},
$$

where we have used the decomposition, $\Lambda^{2}\left(\mathbb{R}^{8}\right)=\Lambda_{\mathbf{7}}^{2} \oplus \Lambda_{\mathbf{2 1}}^{2}$, of the space of 2-forms in $\mathbb{R}^{8}, \Lambda^{2}\left(\mathbb{R}^{8}\right)$, in irreducible $\operatorname{Spin}(7)$ representations. Note that $\Lambda_{\mathbf{2 1}}^{2}=\mathfrak{s p i n}(7)$. As the Killing spinor is $\left(\operatorname{Spin}(7) \ltimes \mathbb{R}^{8}\right) \times \mathbb{R}$ invariant, it satisfies $\Gamma^{-} \epsilon=0$ and $\omega_{i j} \Gamma^{i j} \epsilon=0$ for all $\omega \in \Lambda_{\mathbf{2 1}}^{2}$.

As in the previous cases that the spacetime admits a null 1-form bilinear, we define the space transverse to the lightcone $\mathcal{T}$. $\mathcal{T}$ is further decomposed as $\mathcal{T}=L \oplus \mathcal{Z}$, where $L$ is spanned by $\mathbf{e}^{9}$ and $\mathcal{Z}$ is associated to the spinor representation of $\operatorname{Spin}(7)$. The conditions (293) can be rewritten as

$$
\mathcal{L}_{X} g=0, \quad \mathcal{L}_{X} \epsilon=0, \quad i_{V}(X \wedge d X) \in \Lambda_{\mathbf{2 1}}^{2},
$$

where $\Lambda_{\mathbf{2 1}}^{2}$ now denotes the space of sections of the vector bundle associated with the $\mathbf{2 1}$ representation of $\operatorname{Spin}(7)$ and $V$ is the vector field dual to the 1 -form $\mathbf{e}^{+}$. Similarly, (294) is equivalent to

$$
i_{V}\left(\mathcal{L}_{W} \tau\right) \in \Lambda_{\mathbf{3 5}}^{4}
$$


where $W$ is the vector field dual to the 1 -form $\mathbf{e}^{9}$ and we have used the decomposition $\Lambda^{4}\left(\mathbb{R}^{8}\right)=\Lambda_{35}^{4} \oplus \Lambda_{1}^{4} \oplus \Lambda_{7}^{4} \oplus \Lambda_{27}^{4}$ in irreducible representations of $\operatorname{Spin}(7)$. Note that $\Lambda_{35}^{4}$ is the space of the anti-self-dual 4 -forms. The remaining condition (295) can be rewritten in terms of the Lee form of the $\operatorname{Spin}(7) 4$-form $\phi$ as

$$
\Omega_{9,9 i}-6 \Omega_{i,+-}=-3 \tilde{\theta}_{i},
$$

where $\tilde{\theta}=-\frac{1}{6} \star_{8}\left(\left(\star_{8} \tilde{d} \phi\right) \wedge \phi\right), \star_{8}$ denotes the Hodge dual operation in $\mathcal{Z}$ and $\tilde{d}$ denotes the restriction of the exterior derivative on $\mathcal{Z}$.

\subsection{Geometry of IIA and IIB $N=1$ backgrounds}

The investigation of the geometry of $N=1$ backgrounds in type IIB and IIA $d=10$ supergravities has been carried out in [186-188] and [189-191], respectively, where the explicit solution to the KSEs can be found. Here we shall give a very brief description of the results.

Beginning with IIB supergravity, the gauge group of the theory in the formulation of [192,193] is $\operatorname{Spin}(9,1) \cdot U(1)$. The $U(1)$ arises because the supersymmetry parameter $\epsilon$, which is in the Weyl representation of $\operatorname{Spin}(9,1)$, is additionally twisted with the pull-back of the canonical bundle of the upper-half plane. This is the scalar manifold of the axion and the dilaton, i.e. the two IIB scalars. More detailed description of the couplings of IIB supergravity will be given in Appendix E.2. An investigation reveals that there are three types of orbits of $\operatorname{Spin}(9,1)$ in the space of Weyl spinors with isotropy group either $\operatorname{Spin}(7) \ltimes \mathbb{R}^{8}$, or $S U(4) \ltimes \mathbb{R}^{8}$, or $G_{2}$.

As in the more detailed analysis we have presented in Section 8.2.2 for $d=11$ supergravity, the conditions on the geometry of the IIB $N=1$ backgrounds imposed by the KSEs include the existence of a Killing vector field $X$ which is constructed as a bilinear of the Killing spinor $\epsilon$. In addition, $X$ leaves the other fields invariant as well as the Killing spinor $\epsilon$. One difference is that the spinorial Lie derivative in IIB is defined as in (38) with $\nabla$ given in, i.e. it involves an additional connection term - $(i / 2) Q$ associated with the $U(1)$ twist of $\epsilon . X$ is timelike or null depending on whether the isotropy group is compact or non-compact, respectively. These are the universal conditions expected from the IIB KSEs. In all cases there are additional conditions on the spacetime which depend on the type of orbit to which the Killing spinor $\epsilon$ belongs, for details see the references above.

In (massive) IIA supergravity [194-197] the supersymmetry parameter is in the Majorana representation of $\operatorname{Spin}(9,1)$. One can show that there are four types of orbits of $\operatorname{Spin}(9,1)$ in the space of Majorana spinors with isotropy groups either $\operatorname{Spin}(7), \operatorname{Spin}(7) \ltimes \mathbb{R}^{8}, S U(4)$ or $G_{2} \ltimes \mathbb{R}^{8}$. The solution of the KSEs of IIA supergravity for one Killing spinor gives restrictions on the geometry of the spacetime. These again include the universal conditions. Therefore there is a Killing vector $X$ constructed as a bilinear of the Killing spinor $\epsilon$ which leaves all the fields invariant as well as $\epsilon$, where the spinorial Lie derivative is given as in (38). There are additional conditions on the geometry of spacetime which depend on the type of orbit to which $\epsilon$ belongs and can be found in the original papers mentioned above.

\subsection{Global properties of the solutions}

The description of the geometry of $d=11$ and $d=10$ type II supergravity backgrounds with $N=1$ supersymmetry we have given is local. It depends on the assumption that at some open set of the spacetime the supercovariant connections preserve the type of orbit to which the Killing spinor belongs under parallel transport. This is not automatically the case as the holonomy of the supercovariant connections for generic backgrounds is in $\operatorname{SL}(32, \mathbb{R})$, see also Section 2.2. Therefore, under parallel transport the spinors are transformed with $S L(32, \mathbb{R})$ transformations which do not necessarily preserve their orbit type. The properties of form bilinears also change under such parallel transport. For $N=1$ backgrounds in $d=11$ supergravity an indication that a Killing spinor with isotropy group $S U(5)$ has changed under parallel transport to another one with isotropy group $\operatorname{Spin}(7) \ltimes \mathbb{R}^{9}$ is that the Killing vector bilinear $X$ changes from timelike to null at some region of spacetime. Such phenomenon is widespread in gravitational backgrounds and signals the existence of Killing horizons.

A priori the spinorial geometry method can be adapted to solve this problem. For example one can choose Killing spinor representatives which include all orbit types. However in such a case, the resulting linear system will be rather involved.

A related issue is the restriction of the $G$-structure of the spacetime as a consequence of the existence of a Killing spinor. As a Killing spinor is a no-where vanishing section of an appropriate spin bundle, one expects that the $G$-structure of the spacetime may reduce to a subgroup of the isotropy group of the Killing spinor. However in $d=11$ and $d=10$ type II theories the relevant spin bundle has a rank much larger than the dimension of the spacetime. As a consequence it always admits no-where vanishing sections. So a priori the existence of a no-where vanishing section does not necessarily imply the reduction of the spacetime $G$-structure. However, if one insists that the orbit type of a Killing spinor is preserved under parallel transport everywhere on the spacetime, then the structure group reduces to a subgroup of the isotropy group of the Killing spinor. These observations clarify the use of the $G$-structure language to describe the geometry of supersymmetric backgrounds in $d=11, d=10$ type II and other supergravities. 


\subsection{Killing superalgebras}

The Killing spinors and associated Killing vector bilinears on a supersymmetric background can be endowed with a superalgebra structure. Superalgebras are $\mathbb{Z}_{2}$-graded associative algebras with a compatible bracket structure which satisfies the super-Jacobi identities, see e.g. [198]. In particular, superalgebras decompose as $\mathfrak{g}=\mathfrak{g}_{0}+\mathfrak{g}_{1}$, where $\mathfrak{g}_{0}$ and $\mathfrak{g}_{1}$ are the even and odd subspaces of the superalgebra with grading 0 and 1, respectively. Given elements $\alpha, \beta \in \mathfrak{g}$ with grading $|\alpha|$ and $|\beta|$, the bracket is defined as

$$
[\alpha, \beta]_{\mathfrak{g}}:=\alpha \beta-(-1)^{|\alpha||\beta|} \beta \alpha .
$$

This satisfies the super-Jacobi identities

$$
\left[[\alpha, \beta]_{\mathfrak{g}}, \gamma\right]_{\mathfrak{g}}+(-1)^{|\gamma|(|\alpha|+|\beta|)}\left[[\gamma, \alpha]_{\mathfrak{g}}, \beta\right]_{\mathfrak{g}}+(-1)^{|\alpha|(|\beta|+|\gamma|)}\left[[\beta, \gamma]_{\mathfrak{g}}, \alpha\right]_{\mathfrak{g}}=0 .
$$

Note that the bracket $[\cdot, \cdot]_{\mathfrak{g}}$ between two odd elements of the superalgebra is an anticommutator which we denote with $\{\cdot, \cdot\}$ while all the rest of the brackets are commutators $[\cdot, \cdot]$.

Returning to the definition $[199,200]$ of a Killing superalgebra $\mathfrak{g}$ for supersymmetric backgrounds the odd subspace $\mathfrak{g}_{1}$ of $\mathfrak{g}$ is spanned by $Q_{\epsilon_{\mathbf{n}}}$, where we have associated a generator $Q_{\epsilon_{\mathbf{n}}}$ to every linearly independent Killing spinor $\epsilon_{\mathbf{n}}$ on the spacetime, $\mathbf{n}=1, \ldots, N$. Similarly, $\mathfrak{g}_{0}$ is spanned by $V_{X_{\mathrm{mn}}}$, where we have associated a generator $V_{X_{\mathrm{mn}}}$ to every linearly independent Killing vector bilinear $X_{\mathbf{m n}}$ on the spacetime. For $d=11$ and (massive) IIA supergravities, the latter are defined in terms of the Killing spinors as

$$
X_{\mathbf{m n}}:=\left\langle\left(\Gamma_{+}-\Gamma_{-}\right) \epsilon_{\mathbf{m}}, \Gamma_{A} \epsilon_{\mathbf{n}}\right\rangle \mathbf{e}^{A} .
$$

For IIB supergravity, one takes the real part of the above expression. Note that the inner product used in (301) is proportional to the Dirac inner product, see Appendix B, where the proportionality factor has been introduced for convenience. The proof that all $X_{\mathbf{m n}}$ are Killing follows from the linearity of the KSEs and the Killing property of the vector bilinear of a single Killing spinor that we have already demonstrated, see e.g. Sections 8.2.2 and 8.3 for $d=11$ supergravity. Similar definitions for $X$ exist in all supergravity theories. Observe that $X_{\mathrm{mn}}=X_{\mathrm{nm}}$.

The (anti-)commutators of the Killing superalgebra are defined as follows

$$
\begin{aligned}
& \left\{Q_{\epsilon_{\mathbf{m}}}, Q_{\epsilon_{\mathbf{n}}}\right\}=V_{X_{\mathbf{m n}}}, \quad\left[V_{X_{\mathbf{m} \mathbf{n}}}, Q_{\epsilon_{\mathbf{p}}}\right]=Q_{\mathcal{L}_{X_{\mathbf{m} \mathbf{n}}} \epsilon_{\mathbf{p}},}, \\
& {\left[V_{X_{\mathbf{m} \mathbf{n}}}, V_{X_{\mathbf{p q}}}\right]=V_{\left[X_{\mathbf{m} \mathbf{n}}, X_{\mathbf{p q}}\right]},}
\end{aligned}
$$

where $\left[X_{\mathbf{m n}}, X_{\mathbf{p q}}\right]$ is the Lie commutator of two vector fields, and in $d=11$ and (massive) IIA supergravities $\mathcal{L}_{X_{\mathrm{mn}}}$ is the spinorial Lie derivative (38) with respect to $X_{\mathbf{m n}}$, while in IIB supergravity the spinorial Lie derivative involves an additional $U(1)$ twist as explained in Section 8.4. It has been demonstrated in [146] that the super-Jacobi identities (300) are satisfied in $d=11$ and IIB supergravities. This is expected to hold for all supergravity theories. It is worth pointing out that the universal condition $\mathcal{L}_{X} \epsilon=0$ we have found for $N=1$ backgrounds in all supergravity theories we have investigated is required for the super-Jacobi identity $\left[\left\{Q_{\epsilon}, Q_{\epsilon}\right\}, Q_{\epsilon}\right]=\mathcal{L}_{X} \epsilon=0$ to be satisfied.

\section{Maximally supersymmetric solutions in $d=10$ and $d=11$}

Maximally supersymmetric backgrounds are those that preserve all supersymmetries of a supergravity theory. Typically, these have a special status amongst the other solutions. For example in $d=10$ type II and $d=11$ supergravities, the maximally supersymmetric backgrounds preserve 32 supersymmetries and have found extensive applications in compactifications and in the AdS/CFT correspondence. They have been classified up to a local isometry in [10,201]. Here, we shall summarize the main steps of the proof of the classification theorem.

\section{1. $d=11$ supergravity}

The maximally supersymmetric solutions of $d=11$ supergravity are locally isometric to one of the following solutions

- $A d S_{4} \times S^{7}$ with metric and flux

$$
d s^{2}=\ell^{2} d \stackrel{\circ}{2}^{2}\left(A d S_{4}\right)+4 \ell^{2} d \stackrel{\circ}{2}^{2}\left(S^{7}\right), \quad F= \pm 3 \ell^{3} \operatorname{diol}\left(A d S_{4}\right),
$$

$-A d S_{7} \times S^{4}$ with metric and flux

$$
d s^{2}=\ell^{2} d \dot{s}^{2}\left(A d S_{7}\right)+\frac{1}{4} \ell^{2} d \dot{s}^{2}\left(S^{4}\right), \quad F= \pm \frac{3}{8} \ell^{3} \mathrm{~d} \operatorname{dol}\left(S^{4}\right),
$$

- the plane wave with metric and flux

$$
\begin{aligned}
& d s^{2}=2 d v d u+A_{i j} x^{i} x^{j} d v^{2}+\delta_{i j} d x^{i} d x^{j}, \\
& F=\mu d v \wedge d x^{1} \wedge d x^{2} \wedge d x^{3},
\end{aligned}
$$

with $A=-\frac{\mu^{2}}{36} \operatorname{diag}(4,4,4,1,1,1,1,1,1)$ and $\mu \neq 0$, 
- Minkowski spacetime $\mathbb{R}^{10,1}$ for which $F=0$,

where $d \dot{s}^{2}$ and dvol denote the metrics and volume forms of the indicated spaces with radii normalized to one, respectively, and $\ell \in \mathbb{R}_{>0}$. The $A d S_{4} \times S^{7}$ and $A d S_{7} \times S^{4}$ solutions are of the Freund-Rubin form [202] and have been found in [203] and [204], respectively. The plane wave solution has been given in [205]. Observe that plane wave parameter $\mu$ can be absorbed in a coordinate redefinition. The plane wave solution is a Penrose limit of both the maximally supersymmetric AdS backgrounds of the theory [206].

To prove the above statement observe that maximal supersymmetry implies that the supercurvature of the supercovariant connection, $\mathcal{R}_{M N}=\left[\mathcal{D}_{M}, \mathcal{D}_{N}\right]$, must vanish. Expanding this in skew-symmetric products of gamma matrices as

$$
\mathcal{R}_{M N}=\sum_{k=1}^{5} \frac{1}{k !} T_{M N, A_{1} \ldots A_{k}} \Gamma^{A_{1} \ldots A_{k}},
$$

all components of $\mathcal{R}$ in the Clifford algebra basis must vanish, i.e.

$$
T_{M N, A_{1} \ldots A_{k}}=0 .
$$

The explicit expression for these components is given in (E.3).

The vanishing of the term of $\mathcal{R}$ linear in gamma matrices implies that $F \wedge F=0$. In turn this gives

$$
i_{X} F \wedge F=0,
$$

for any spacetime vector field $X$.

Substituting (308) into the vanishing condition of the term cubic in gamma matrices, one finds that

$$
\nabla_{B} F_{C A_{1} A_{2} A_{3}}-\nabla_{C} F_{B A_{1} A_{2} A_{3}}=0 .
$$

This together with the Bianchi identity for $F, d F=0$, gives that

$$
\nabla_{B} F_{A_{1} A_{2} A_{3} A_{4}}=0 \text {. }
$$

Substituting this into the quadratic component, one concludes that the Riemann tensor is also parallel, $\nabla R=0$, and therefore the spacetime of maximally supersymmetric backgrounds is a Lorentzian symmetric space.

After some computation, the terms in quartic gamma matrices imply that

$$
F_{C D\left[A_{1} A_{2}\right.} F_{\left.A_{3} A_{4}\right]}^{C D}=0 \text {. }
$$

Using the results that arise from the terms quartic in gamma matrices, the terms quintic in gamma matrices give

$$
i_{X} F \wedge i_{Y} F=0,
$$

for any vector fields $X, Y$.

Using (308) and (312), one can show that

$$
i_{Y} i_{Z} F \wedge F=0,
$$

for any vector field $Y, Z$. Taking the inner derivation with respect to another vector field of both (312) and (313), one finds that

$$
\begin{aligned}
& i_{X} i_{Y} F \wedge i_{Z} F-i_{X} i_{Z} \wedge i_{Y} F=0, \\
& i_{Z} i_{X} i_{Y} F \wedge F+i_{X} i_{Y} F \wedge i_{Z} F=0,
\end{aligned}
$$

after an appropriate lexicographic relabelling of the vector fields, respectively. The first equation implies that $i_{X} i_{Y} F \wedge i_{Z} F$ is symmetric in the vector field $Z$ and $Y$, while the second implies that it is skew symmetric. As a result the two terms of the second equation in (314) vanish separately. So in particular, one has

$$
i_{Z} i_{X} i_{Y} F \wedge F=0 .
$$

This condition is known as a Plücker relation and it implies that $F$ is decomposable, i.e. it can be written as the wedge product of four one forms

$$
F=\theta^{1} \wedge \theta^{2} \wedge \theta^{3} \wedge \theta^{4} .
$$

As a result $F$ determines a 4-plane at every point in spacetime.

As we have already mentioned the spacetime is a Lorentzian symmetric spaces, $M=G / H$. In particular, $\mathfrak{g}=\mathfrak{h} \oplus \mathfrak{m}$, and $\mathfrak{m}$ is identified as the tangent space of $G / H$ at the origin. Moreover, the Lorentzian symmetric space have been classified. It can be shown that they are products of one of the Lorentzian spaces Minkowski $\mathbb{R}^{n-1,1}, \mathrm{dS}_{n}, \operatorname{AdS}_{n}$ or Cahen-Wallach $\mathrm{CW}_{n}(A)$ with a Euclidean symmetric space [21]. In particular, the metric of Cahen-Wallach spaces is given in (C.10), Appendix C.2, with $\operatorname{det} A \neq 0$. 
To continue, since $F$ is decomposable and parallel, it spans an $H$-invariant four-plane $\mathfrak{n} \subset \mathfrak{m}$. If $F$ is either time-like or space-like, then the normal $\mathfrak{n}^{\perp}$ is also $H$-invariant and the symmetric space decomposes into a product of a four-dimensional and a seven-dimensional symmetric space, $M=X_{4} \times Y_{7}$. Using this, and solving the equation quadratic in gamma matrices (306), one finds the $A d S_{4} \times S^{7}$ and $A d S_{7} \times S^{4}$ solutions for $F$ time-like and space-like, respectively, as stated in the beginning of the section.

It remains to investigate the case in which $F$ is null. The only symmetric spaces that admit parallel null forms are those that locally are products $\mathrm{CW} \times N$, where $N$ is a Euclidean symmetric space. The equation quadratic in gamma matrices (306) implies that the only option is the plane wave solution. The Minkowski space arises whenever $F=0$. This completes the proof.

\subsection{IIB supergravity}

The maximally supersymmetric solutions of IIB supergravity are locally isometric to one of the following:

- $A d S_{5} \times S^{5}$ with non-vanishing fields

$$
\begin{aligned}
& d s^{2}=\ell^{2} d \dot{s}^{2}\left(\operatorname{AdS}_{5}\right)+\ell^{2} d \dot{s}^{2}\left(S^{5}\right), \\
& F= \pm \ell^{4}\left(\operatorname{dvol}\left(\operatorname{AdS}_{5}\right)-\operatorname{dinol}\left(S^{5}\right)\right),
\end{aligned}
$$

- the plane wave solution with non-vanishing fields

$$
\begin{aligned}
& d s^{2}=2 d v d u+A_{i j} x^{i} x^{j} d v^{2}+\delta_{i j} d x^{i} d x^{j}, \quad A=-\mu^{2} \mathbf{1}, \\
& F=\mu d v \wedge\left(d x^{1} \wedge d x^{2} \wedge d x^{3} \wedge d x^{4}+d x^{5} \wedge d x^{6} \wedge d x^{7} \wedge d x^{8}\right),
\end{aligned}
$$

- Minkowski space $\mathbb{R}^{9,1}$,

where $\ell \in \mathbb{R}_{>0}$ and $d \grave{s}^{2}$ and diol denote the metrics and volume forms of the corresponding spaces with radii normalized to one, respectively. The existence of a IIB maximally supersymmetric $A d S_{5} \times S^{5}$ solution has been mentioned in [193], see also the comment added there. The plane wave solution has been found in [207] and has been demonstrated in [206] to be the Penrose limit of the AdS solution. The parameter $\mu \neq 0$ of the plane wave solution can be absorbed via a coordinate redefinition.

The proof for this proceeds as in $d=11$. The algebraic Killing spinor equation of IIB supergravity implies that

$$
P=G=0,
$$

i.e. the one-form and three-form field strengths vanish. To investigate the gravitino KSE, we again consider the supercovariant curvature, $\mathcal{R}$, with only five-form flux. Expanded in skew-symmetric products of gamma matrices, $\mathcal{R}$ is written as

$$
\mathcal{R}_{M N}=\sum_{k=0}^{2} \frac{1}{(2 k) !} T_{M N, A_{1} \ldots A_{2 k}} \Gamma^{A_{1} \ldots A_{2 k}} .
$$

Again maximal supersymmetry requires that

$$
T_{M N, A_{1} \ldots A_{2 k}}=0 \text {. }
$$

The condition that arises for $k=0$, together with (319), implies that the dilaton and axion can be taken to be constant. The term quartic in gamma matrices implies that

$$
\begin{aligned}
\nabla_{B} F_{C A_{1} \ldots A_{4}}-\nabla_{C} F_{B A_{1} \ldots A_{4}} & =0, \\
F_{D B\left[A_{1} A_{2} A_{3}\right.} F_{\left.A_{4} A_{5} A_{6}\right] C}{ }^{D} & =0 .
\end{aligned}
$$

The first equation together with the Bianchi identity for $F$ implies that $F$ is parallel

$$
\nabla_{B} F_{A_{1} \ldots A_{5}}=0 \text {. }
$$

The second equation can also be written as

$$
i_{X} F_{L} \wedge i_{Y} F^{L}=0 .
$$

Observe that this also implies that $i_{X} F_{L} \wedge F^{L}=0$. Then a similar argument to that presented for eleven-dimensional supergravity reveals that

$$
i_{Z} i_{Y} i_{X} F_{L} \wedge F^{L}=0 .
$$

This relation is not a Plücker relation but a generalization. It has been solved in [201] to reveal that there is a decomposable five-form $K$ such that $F=K+\star K$, where $K$ is a simple form. Note that (325) and its generalization to (k+1)-forms can also be thought as the Jacobi identity of metric k-Lie algebras [208]. 
It remains to solve the condition quadratic in gamma matrices (320). This together with $\nabla F=0$ implies that the spacetime is a symmetric space, $G / H$. Moreover, if $K$ is either time-like or space-like, then $F$ defines an $H$-invariant fivedimensional subspace $\mathfrak{n}$ of $\mathfrak{m}$ which has an $H$-invariant normal $\mathfrak{n}^{\perp}, \mathfrak{g}=\mathfrak{h} \oplus \mathfrak{m}$. Again the spacetime decomposes and the only solution is $A d S_{5} \times S^{5}$. The remaining case is when $K$ is null. This gives the plane wave solution. The Minkowski space arises whenever all form field strengths vanish. This completes the proof.

\subsection{Other $d=10$ supergravities}

A similar analysis to the one presented above for the $d=11$ and IIB supergravities reveals that the maximally supersymmetric backgrounds of IIA supergravity are locally isometric to $\mathbb{R}^{9,1}$ with constant dilaton and with all remaining form field strengths vanishing. The same applies to the heterotic or type I supergravities. The massive IIA supergravity does not have a maximally supersymmetric background provided that the cosmological constant is non-zero.

\section{Nearly maximally supersymmetric supergravity backgrounds}

Spinorial geometry can be adapted to classify backgrounds that preserve a near maximal number of supersymmetries. In particular, we shall present a brief description of the proof that the $N=31$ backgrounds of IIB and $d=11$ supergravities are locally maximally supersymmetric [60,209]. A similar result for IIA supergravity has been demonstrated in [210].

To investigate the geometry of backgrounds of $d=10$ and $d=11$ supergravities with a near maximal number of supersymmetries it is more convenient to use the gauge symmetry to choose a canonical form for the normals to the Killing spinors. To see this, let us specialize to the $N=31$ case, and write the Killing spinors as,

$$
\epsilon^{r}=\sum_{i=1}^{32} f_{i}^{r} \eta^{i}, \quad r=1, \ldots, 31,
$$

where $\eta^{i}$ is a basis in the space of spinors and $f_{i}^{r}$ is a matrix of real spacetime functions of rank 31 . The main difficulty in solving the KSEs or their integrability conditions is that $f_{i}^{r}$ is not a square invertible matrix. To overcome this, one uses the gauge symmetry of the KSEs to choose the hyperplane of Killing spinors. It turns out that the most efficient way to do this is to use the gauge symmetry to orient the normal $v$ to the Killing spinors into a particular direction. Having chosen the normal spinor $v$, the 31 Killing spinors are then defined by the orthogonality condition

$$
\left\langle\nu, \epsilon^{r}\right\rangle_{s}=0,
$$

where $\langle\cdot, \cdot\rangle_{s}$ is a suitable Spin-invariant inner product in the space of spinors. Typically, there are several cases that one should investigate corresponding to the number of canonical forms for $v$ up to supergravity gauge transformations, i.e. the number of orbit types of the gauge group on the space of spinors. Although the methodology to find nearly maximally supersymmetric backgrounds here is described in the context of $d=10$ and $d=11$ supergravities, it also applies to all other theories.

\section{1. $N=31, I I B$}

To begin the proof of the main result in IIB supergravity, a convenient basis in the space of IIB spinors can be chosen as $\left(\eta^{p}, i \eta^{p}\right)$, where $\eta^{p}$ is a basis in the space of Majorana-Weyl spinors. In such a case, the Killing spinors can be written as

$$
\epsilon^{r}=\sum_{p=1}^{16} f_{p}^{r} \eta^{p}+i \sum_{p=1}^{16} f_{16+p}^{r} \eta^{p},
$$

where $\left(f_{p}^{r}, f_{16+p}^{r}\right)$ is a matrix of real spacetime functions of rank 31 . A choice of a $\operatorname{Spin}(9,1)$-invariant inner product is the real part of the Majorana inner product of IIB spinors

$$
\left\langle\epsilon_{1}, \epsilon_{2}\right\rangle_{s}:=\operatorname{ReB}\left(\epsilon_{1}, \epsilon_{2}\right),
$$

see Appendix B. It turns out that B is skew-symmetric and vanishes when restricted to either chiral or anti-chiral spinors. As a result, two spinors have a non-trivial inner product iff one of the spinors is chiral and the other anti-chiral. Therefore, since the IIB Killing spinors are chosen to be chiral, the normal $v$ lies in the anti-chiral representation of $\operatorname{Spin}(9,1)$.

The gauge group $\operatorname{Spin}(9,1)$ has three different orbits in the space of anti-chiral spinors with representatives

$$
\begin{aligned}
& (n+i m)\left(e_{5}+e_{12345}\right), \quad(n-\ell+i m) e_{5}+(n+\ell+i m) e_{12345}, \\
& n\left(e_{5}+e_{12345}\right)+i m\left(e_{1}+e_{234}\right)
\end{aligned}
$$

and with isotropy groups $\operatorname{Spin}(7) \ltimes \mathbb{R}^{8}, S U(4) \ltimes \mathbb{R}^{8}$ and $G_{2}$, respectively. Therefore there are three different choices for the normal $v$ to the 31 Killing spinors. 
The analysis for the three different cases is similar. Because of this, we shall outline the proof for the first normal spinor and the details for the other two cases can be found in [60]. Substituting the first spinor in (330) as a normal and the expression for the Killing spinors (328) into the orthogonality condition (327), one finds

$$
f_{1}^{r} n-f_{17}^{r} m=0 .
$$

After assuming without loss of generality that $n \neq 0$ and solving this equation for $f_{r}^{1}$, one finds that the Killing spinors (328) can be written as

$$
\epsilon^{r}=\frac{f_{17}^{r}}{n}(m+i n)\left(1+e_{1234}\right)+\sum_{k \neq 1,17} f_{k}^{r} \eta^{k}
$$

Observe now that the transformation from the Killing spinors $\left(\epsilon^{r}\right)$ to the basis $\left((n+i m)\left(1+e_{1234}\right), \eta^{k}\right)$ is invertible. Substituting this into the algebraic KSE of IIB supergravity, see Appendix E.2, one finds that

$$
P_{M} \Gamma^{M} \eta^{p}=0, \quad p=2,3, \ldots, 16 .
$$

This is due to the complex conjugation operation in the algebraic KSE and the choice of the basis in (332). The above equation implies that $P$ must be null. But some of the $\eta^{p}$ spinors are annihilated by $\Gamma^{-}$and some others are annihilated by $\Gamma^{+}$. As a result the only solution that satisfies both light-cone projections is $P=0$.

Next, if $P=0$, the algebraic KSE is linear over the complex numbers, as a result it has an even number of solutions. Since it is required to have 31 , one concludes that it should have 32 . The only Clifford algebra element which annihilates all spinors is the zero element and thus the 3-form flux vanishes, $G=0$.

Therefore the algebraic KSE gives $P=G=0$. If this is the case, then the gravitino KSE becomes linear over the complex numbers, and therefore admits an even number of solutions. So if it is required to admit 31 Killing spinors, then it will have 32. The same analysis holds for the other two normal spinors in (330), see [60] and therefore it follows that all IIB backgrounds with 31 supersymmetries are maximally supersymmetric.

10.2. $N=31, D=11$

As in the IIB case outlined in the previous section, $d=11$ backgrounds with 31 supersymmetries are also maximally supersymmetric [209]. The proof in $d=11$ though is different from that described for IIB. This is because one has to solve directly the gravitino KSE. In particular, one has to show that for the backgrounds with 31 supersymmetries the integrability condition of the gravitino KSE, $\mathcal{R} \epsilon^{r}=0$, implies that the supercovariant curvature vanishes, $\mathcal{R}=0$.

To continue, it is convenient to write $\mathcal{R}$ in terms of two different bases. In one of the bases, $\mathcal{R}$ automatically satisfies $\mathcal{R} \epsilon^{r}=0$. While in the other, one can easily impose the field equations and Bianchi identities of supergravity theories. Comparing the two expressions, one can show the vanishing of the supercovariant curvature.

To proceed further, let $\left(\eta^{i}\right)$ be a basis in the space of spinors. Then observe that the supercovariant curvature for a background with 31 Killing spinors can be written as

$$
\mathcal{R}_{M N}=\sum_{i=1}^{32} u_{M N, i} \eta^{i} \otimes v,
$$

where the $u$ 's are spacetime forms, the spinor indices have been suppressed and $v$ is the normal to the Killing spinors. The orthogonality condition has been taken with respect to a $\operatorname{Spin}(10,1)$-invariant Majorana inner product. In particular, $\mathcal{R}_{M N} \epsilon^{r}=0$ as required. Therefore in terms of the $u$ 's the supercovariant curvature satisfies all the supersymmetry conditions.

To constrain further the components $u$ of $\mathcal{R}$, one has to impose the field equations and Bianchi identities of 11 -dimensional supergravity. These are most easily expressed in terms of the $T$ components. In particular, observe that $\Gamma^{N} \mathcal{R}_{\mathrm{MN}}$ is a linear combination of field equations and Bianchi identities, and therefore it necessarily vanishes identically. In turn this leads to

$$
\begin{aligned}
& \left(T_{M N}^{1}\right)^{N}=0, \quad\left(T_{M N}^{2}\right)_{P}{ }^{N}=0, \quad\left(T_{M P_{1}}^{1}\right)_{P_{2}}+\frac{1}{2}\left(T_{M N}^{3}\right)_{P_{1} P_{2}}{ }^{N}=0, \\
& \left(T_{M\left[P_{1}\right.}^{2}\right)_{\left.P_{2} P_{3}\right]}-\frac{1}{3}\left(T_{M N}^{4}\right)_{P_{1} P_{2} P_{3}}{ }^{N}=0,\left(T_{M\left[P_{1}\right.}^{3}\right)_{\left.P_{2} P_{3} P_{4}\right]}+\frac{1}{4}\left(T_{M N}^{5}\right)_{P_{1} \ldots P_{4}}{ }^{N}=0 \text {, } \\
& \left.\left(T_{M\left[P_{1}\right.}^{4}\right)_{P_{2} \cdots P_{5}}\right]-\frac{1}{5 \cdot 5 !} \epsilon_{P_{1} \cdots P_{5}} Q_{1} \cdots Q_{6}\left(T_{M Q_{1}}^{5}\right)_{Q_{2} \cdots Q_{6}}=0 .
\end{aligned}
$$

The second and third of these equations are consequences of the Einstein and $F$ field equations, respectively. We also use the additional conditions

$$
\left(T_{M N}^{1}\right)_{P}=\left(T_{[M N}^{1}\right)_{P]}, \quad\left(T_{M N}^{2}\right)_{P Q}=\left(T_{P Q}^{2}\right)_{M N}, \quad\left(T_{[M N}^{3}\right)_{P Q R}=0,
$$

which can easily be derived by inspecting the explicit expressions of $T$ in terms of the physical fields in (E.3) and by using the Bianchi identity of $F$. Observe that the first condition in (335) is a consequence of the first condition in (336).

Next comparing (334) with (306), one concludes that

$$
\left(T_{M N}^{k}\right)_{A_{1} A_{2} \ldots A_{k}}=\frac{(-1)^{k+1}}{32} u_{M N, i} \mathrm{~B}\left(\eta^{i}, \Gamma_{A_{1} A_{2} \ldots A_{k}} \nu\right), \quad k=0, \ldots, 5,
$$


where the relation

$$
\eta \otimes \theta=\frac{1}{32} \sum_{k=0}^{5} \frac{(-1)^{k+1}}{k !} \mathrm{B}\left(\eta, \Gamma_{A_{1} A_{2} \ldots A_{k}} \theta\right) \Gamma^{A_{1} A_{2} \ldots A_{k}},
$$

of bi-spinors to spacetime forms has been used. Since $T^{0}$ vanishes identically, consistency requires that the $u$ 's must satisfy

$$
\sum_{i=1}^{32} u_{M N, i} B\left(\eta^{i}, v\right)=0
$$

This equation is easily solved by choosing an appropriate basis $\left(\eta^{i}\right)$ and setting one of the $u$ 's to zero.

It remains to impose the conditions (335) and (336) on the $u$ 's. For this one uses the relation (337) and a representative for the normal spinor $v$ up to $\operatorname{Spin}(10,1)$ transformations. As the normal spinors are in the same representation as the Killing spinors, and as $\operatorname{Spin}(10,1)$ has two different orbits in $\Delta_{32}$ with isotropy groups $S U(5)$ and $\operatorname{Spin}(7) \ltimes \mathbb{R}^{9}$, there are two different cases of backgrounds with 31 supersymmetries to be investigated. We shall not proceed further to carry out the analysis as it is rather technical and can be found in [209]. The key point to stress though is that the proof requires the use of the field equations and Bianchi identities in addition of course to the requirement that the backgrounds preserve 31 supersymmetries.

The possibility remains that backgrounds with 31 supersymmetries can be constructed as discrete quotients of maximally supersymmetric backgrounds. This possibility has been excluded in [61]. Therefore all $d=11$ backgrounds with 31 supersymmetries are maximally supersymmetric.

\section{3. $N>16$ supersymmetric backgrounds}

In IIB supergravity, one can show that all $N>28$ backgrounds are maximally supersymmetric [211]. Moreover there is a unique $N=28$ plane wave solution [212]. This is a superposition of the maximally supersymmetric plane wave and a common sector solution which preserves 28 supersymmetries [213].

In $d=11$ supergravity, the results are less stringent. It can be shown though that all backgrounds that preserve $N \geq 30$ supersymmetries are maximally supersymmetric [214]. As has been mentioned all $N=31$ IIA supergravity backgrounds are maximally supersymmetric [210]. It is likely that one can obtain in IIA supergravity stronger results similar to those of IIB. This is because apart from the gravitino KSE, the theory has an algebraic KSE and therefore the techniques used for IIB can be applied in IIA. However no such investigation has taken place. It should be noted that there are several solutions known, all plane waves, that preserve $16<N<32$ supersymmetries in $d=10$ and $d=11$ supergravities but these have not been systematically constructed.

\subsection{The homogeneity theorem}

The homogeneity conjecture states the following.

- All supergravity backgrounds that preserve more than half of the supersymmetry of a theory are locally isometric to Lorentzian homogeneous spaces.

The conjecture has been confirmed [67] for $d=11$ and type II $d=10$ supergravities. So all backgrounds of these theories that preserve $N>16$ supersymmetries are locally isometric to $d=11$ and $d=10$ Lorentzian homogeneous spaces, respectively. The proof of this result is remarkably simple and can be demonstrated as follows.

The aim of the proof is to show that if a background preserves more than half of the supersymmetry of a supergravity theory, then its tangent space at every point will be spanned by the Killing vectors constructed as bilinears of Killing spinors. As a result, it admits a transitive group action of isometries. The calculation can be done point-wise on the spacetime $M$. For this consider two Killing spinors $\epsilon$ and $\eta$ and the Killing vector bilinear

$$
\left.X\right|_{p}=\left.\left\langle\epsilon, \Gamma^{A} \eta\right\rangle_{s} \partial_{A}\right|_{p}
$$

evaluated at a point $p \in M$, where $\langle\cdot, \cdot\rangle_{s}$ is a suitable spin invariant inner product over $\mathbb{R}$ such that the above bilinear is associated to a Killing vector field on $M$. Such a bilinear always exists in supergravity theories and the particular choice is not relevant for the argument that follows.

Let us identify the tangent and co-tangent bundles using the spacetime metric. If for all Killing spinors $\epsilon$ and $\eta$ the bilinears (340) span $T_{p} M$ at every $p \in M$, there is nothing to show. Suppose instead that they do not. In such a case, there is a vector field $Y, Y_{p} \neq 0$, which is normal to the span of all bilinears and therefore

$$
\left.\left(Y^{A} X_{A}\right)\right|_{p}=\left.\left\langle\epsilon, Y^{A} \Gamma_{A} \eta\right\rangle_{s}\right|_{p}=0 .
$$

The last relation implies that the Clifford algebra operation $\left.Y^{A} \Gamma_{A}\right|_{p}$ is a map from the bundle of Killing spinors $\mathcal{K}$ to its normal $\mathcal{K}^{\perp},\left.Y^{A} \Gamma_{A}\right|_{p}:\left.\mathcal{K}_{p} \rightarrow \mathcal{K}\right|_{p} ^{\perp} . \mathcal{K}$ is a subbundle of the spin bundle of the supergravity theory whose fibre at every point $p, \mathcal{K}_{p}$, is spanned by the Killing spinors at that point and the normal $\mathcal{K}^{\perp}$ is taken with respect to $\langle\cdot, \cdot\rangle_{s}$. 
However $\left(Y^{A} \Gamma_{A}\right)^{2}=Y^{2} \mathbf{1}$. This implies that if $\left.Y\right|_{p}$ is either timelike or spacelike, $\left.\left(Y^{A} \Gamma_{A}\right)\right|_{p}$ is an injection as the kernel is $\{0\}$. But if the solutions preserve more than half of the supersymmetry, this is in conflict with the assumption that $\operatorname{rank} \mathcal{K}>\operatorname{rank} \mathcal{K}^{\perp}$. Therefore $\left.Y\right|_{p}=0$ and the bilinears (340) $\operatorname{span} T_{p} M$.

It remains to investigate the possibility that $\left.Y\right|_{p}$ is null with $\left.Y\right|_{p} \neq 0$. Focusing on the $d=11$ and $d=10$ type II supergravities at hand, if a solution preserves more than half of the supersymmetry, then at least one of the bilinears (340) will have to be timelike. The maximal number of linearly independent Killing spinors that can give only null vector bilinears is 16. The presence of an additional Killing spinor, which is the case here as $N>16$, will give rise to a timelike vector bilinear. This becomes rather apparent after looking at the description of the relevant spinor representations in terms of forms. As a result, the normal $Y$ to the span of the bilinears (340) cannot be null because then it cannot be orthogonal to the timelike Killing vector bilinears. This is a contradiction of our assumption that $\left.Y\right|_{p}$ is null and therefore we must set again $\left.Y\right|_{p}=0$. This proves that all backgrounds of $d=11$ and $d=10$ type II supergravities theories that preserve strictly more than 16 supersymmetries must locally be Lorentzian homogeneous spaces. This result also applies to heterotic supergravity and is expected to hold to many other theories as well.

\section{Horizons}

\subsection{Symmetry enhancement near black hole and brane horizons}

A key phenomenon which has spearheaded many of the most well-known examples of the AdS/CFT correspondence is that there is a (super)symmetry enhancement near certain black-hole and brane horizons, see e.g. [215,216]. In particular the near horizon geometry of the extreme Reissner-Nordström black hole is $A d S_{2} \times S^{2}$. So the $\mathbb{R} \times S O(3)$ isometry group of the black hole solution enhances near the horizon to $S L(2, \mathbb{R}) \times S O(3)$. The observation that the near horizon geometry of D3branes is $A d S_{5} \times S^{5}$ has led to the most celebrated example of the $A d S_{5} / C F T_{4}$ correspondence which states that string theory on $A d S_{5} \times S^{5}$ is dual to the (maximally supersymmetric) $\mathcal{N}=4 d=4$ gauge theory. The isometry group $S O(4,2) \times S O(6)$ of $A d S_{5} \times S^{5}$ is identified with the product of the conformal times the R-symmetry groups of the gauge theory.

To illustrate how symmetry enhances near horizons, consider the Reissner-Nordström black hole with mass $M$ and charge $Q$. The metric can be written as

$$
d s^{2}=-\frac{\Lambda}{\rho^{2}} d t^{2}+\rho^{2} \Lambda^{-1} d \rho^{2}+\rho^{2} d s^{2}\left(S^{2}\right),
$$

where $\Lambda=\rho^{2}-2 M \rho+Q^{2}=\left(\rho-\rho_{+}\right)\left(\rho-\rho_{-}\right)$and $\rho_{ \pm}=M \pm \sqrt{M^{2}-Q^{2}}$ are the radii of inner and outer horizons. Introduce Eddington-Finkelstein coordinates as $d \rho^{*}=\rho^{2} \Lambda^{-1} d \rho$ and $u=t+\rho^{*}$ to rewrite the metric as

$$
d s^{2}=-\frac{\Lambda}{\rho^{2}} d u^{2}+2 d u d \rho+\rho^{2} d s^{2}\left(S^{2}\right) .
$$

Next, define the coordinate $r=\rho-\rho_{+}$centred at the outer horizon and observe that the metric is analytic in $r$. Expanding around $r=0$, one has

$$
\begin{aligned}
d s^{2} & =2 d u\left[d r-\frac{1}{2}\left(r \frac{\rho_{+}-\rho_{-}}{\rho_{+}^{2}}+r^{2} \frac{2 \rho_{-}-\rho_{+}}{\rho_{+}^{3}}+\mathcal{O}\left(r^{3}\right)\right) d u\right] \\
& +\left(\rho_{+}^{2}+2 r \rho_{+}+r^{2}\right) d s^{2}\left(S^{2}\right) .
\end{aligned}
$$

The linear term in $r$ is the surface gravity of the horizon. The 2-sphere $S^{2}$ is the "spatial horizon section" of the horizon. For an extreme black hole, $\rho_{-}=\rho_{+}$, one can scale the coordinates as $u \rightarrow \ell^{-1} u$ and $r \rightarrow \ell r$ and take the limit $\ell \rightarrow 0$ to find

$$
d s^{2}=2 d u\left[d r-\frac{1}{2} r^{2} \frac{1}{\rho_{+}^{2}} d u\right]+\rho_{+}^{2} d s^{2}\left(S^{2}\right),
$$

which is a metric on $A d S_{2} \times S^{2}$. The geometry in the limit is the "near horizon geometry" of the extreme black hole. For non-extreme black holes, the limit $\ell \rightarrow 0$ diverges and therefore the notion of a near horizon geometry is not well defined.

As has already been mentioned, the $\mathbb{R} \times S O(3)$ isometry group of the Reissner-Nordström black hole in the limit enhances to $S L(2, \mathbb{R}) \times S O(3)$. In addition, viewing the extreme Reissner-Nordström black hole as a solution of the $\mathcal{N}=2 d=4$ minimal supergravity, the $N=4$ supersymmetry of the solution also enhances to $N=8$ near the horizon. The emergence of the conformal group $S L(2, \mathbb{R})$ has been extensively utilized in the microscopic counting of black hole entropy, see e.g. [217].

\subsection{The horizon conjecture}

Before, we proceed to state the horizon conjecture in detail, let us describe a model of a spacetime with an extreme Killing horizon and its near horizon geometry. Killing horizons are spacetime hypersurfaces where a timelike Killing vector field becomes null. The event horizons of many of the black holes of interest are Killing horizons. In fact, under some natural assumptions all the event horizons of $d=4$ black holes are Killing [218]. In what follows the focus will be on the metric but the analysis can be extended to include other fields such as the form fluxes of supergravity theories. It has been shown 
in $[219,220]$ that near a smooth extreme Killing horizon, one can adapt a coordinate system such that the metric takes the form

$$
d s^{2}=2 d u\left(d r+r h_{I}(r, y) d y^{I}-\frac{1}{2} r^{2} \Delta(r, y) d u\right)+\gamma_{I J}(y, r) d y^{I} d y^{J} .
$$

For $\Delta>0, \partial_{u}$ is a timelike Killing vector field which becomes null at the hypersurface $r=0$. The near horizon geometry of the spacetime is defined after scaling the coordinates $u, r$ as $u \rightarrow \ell^{-1} u, r \rightarrow \ell r$, and then taking the limit $\ell \rightarrow 0$. The resulting metric is

$$
d s^{2}=2 d u\left(d r+r h_{I}(y) d y^{I}-\frac{1}{2} r^{2} \Delta(y) d u\right)+\gamma_{I J}(y) d y^{I} d y^{J},
$$

where $h, \Delta$ and $\gamma$ have been evaluated at $r=0$ and therefore they depend only on the $y$ coordinates. This is a metric on an open neighbourhood $M$ of the horizon hypersurface. The co-dimension two subspace $\mathcal{S}$ defined by $u=r=0$ is the "spatial horizon section" of the Killing horizon and it is equipped with the metric $d \tilde{s}^{2}(\mathcal{S})=\gamma_{I J} d y^{I} d y^{J}$. For black hole horizons, $\mathcal{S}$ is expected to be compact without boundary.

If the original spacetime with the Killing horizon is a solution of the Einstein equations, then this will also be the case for $M$ with the near horizon metric (347). Because of this, one can consider $M$ independently of the "parent" spacetime as a solution of the theory. This is the approach that will be adopted from now on in the analysis that follows.

Let $M$ be a spacetime with metric (347), and possibly non-trivial fluxes, that solves the field equations of a supergravity theory and preserves at least one supersymmetry. In addition, assume that the fields are smooth and the spatial horizon section $\mathcal{S}$ is compact without boundary. Then the horizon conjecture [221,222] states the following.

- The number of Killing spinors $N$ of $M$ are

$$
N=2 N_{-}+\operatorname{Index}\left(D_{E}\right),
$$

where $N_{-} \in \mathbb{Z}_{>0}$ and $D_{E}$ is a Dirac operator, defined on the horizon sections $\mathcal{S}$, which is possibly twisted with vector bundle $E$. The choice of $E$ depends on the gauge symmetries of the supergravity theory.

- If $M$ has non-trivial fluxes and $N_{-} \neq 0$, then $M$ will admit an $\mathfrak{s l}(2, \mathbb{R})$ isometry subalgebra.

The conjecture has been proven for various theories which include $d=11$ [221], (massive) IIA [223,224] , IIB [222] and heterotic supergravities [225]. It has also been demonstrated for the minimal gauged $\mathcal{N}=1 d=5$ supergravity [226], the $\mathcal{N}=2 d=4$ gauged supergravity coupled to any number of vector fields [227] and the $\mathcal{N}=1 d=5$ supergravity coupled to any number of vector fields [228].

We shall demonstrate the proof of the horizon conjecture in $d=11$ supergravity but before we do this let us first explain some of its consequences. First, if the index vanishes, $\operatorname{Index}\left(D_{E}\right)=0$, which is the case for non-chiral theories, then $N$ is even. In particular, all odd dimensional near horizon geometries preserve an even number of supersymmetries. Therefore if a near horizon geometry preserves one supersymmetry, possibly inherited from the parent spacetime, it will necessarily preserve another one, and therefore it will exhibit supersymmetry enhancement.

The near horizon geometries with non-trivial fluxes of all non-chiral supergravity theories admit an $\mathfrak{s}(2, \mathbb{R})$ isometry subalgebra. Observe that the near horizon geometry (347) admits two Killing vector fields $\partial_{u}$ and $u \partial_{u}-r \partial_{r}$. Their Lie bracket algebra is solvable. The conjecture states that there must be an additional isometry such that all three together generate $\mathfrak{s l}(2, \mathbb{R})$. Therefore, all such near horizon geometries exhibit symmetry enhancement.

On the other hand if $N_{-}=0$, then $N=\operatorname{index}\left(D_{E}\right)$. The number of Killing spinors is determined by the topology of $\mathcal{S}$. It turns out that such near horizon geometries are rather restricted. Typically all the form field strengths vanish and the scalars are constant. Such near horizon geometries, up to discrete identifications, are products of the form $\mathbb{R}^{1,1} \times \mathcal{S}$, where $\mathcal{S}$ is a product of Berger manifolds that admit parallel spinors. The formula $N=\operatorname{index}\left(D_{E}\right)$ becomes a well-known relation between the index of the Dirac operator and the number of parallel spinors on certain Berger type of manifolds.

\subsection{Proof of the conjecture in $d=11$}

\subsubsection{Preliminaries}

Consider a solution of $d=11$ supergravity with a Killing horizon that satisfies all the assumptions made for the validity of the horizon conjecture. The near horizon geometry apart from the metric also exhibits a non-trivial 4-form flux $F$. The near horizon fields are

$$
d s^{2}=2 \mathbf{e}^{+} \mathbf{e}^{-}+d s^{2}(\mathcal{S}), \quad F=\mathbf{e}^{+} \wedge \mathbf{e}^{-} \wedge Y+r \mathbf{e}^{+} \wedge d_{h} Y+Z,
$$

where $Y$ and $Z$ are a 2 -form and a 4 -form on $\mathcal{S}$, respectively, which depend only of the coordinates $y, d_{h} Y=d Y-h \wedge Y$, and

$$
\mathbf{e}^{+}=d u, \quad \mathbf{e}^{-}=d r+r h-\frac{1}{2} r^{2} \Delta d u, \quad \mathbf{e}^{i}=e_{J}^{i} d y^{J},
$$

is a coframe with $d \tilde{s}^{2}(\mathcal{S})=\delta_{i j} e_{l}^{i} e_{J}^{j} d y^{I} d y^{J}$. Clearly the metric is of the form in (347). 
The Bianchi identities (E.7) and field equations (E.6) of the flux $F$ are rewritten in terms of the horizon fields as

$$
d Z=0, \quad d_{h} \star_{9} Z-\star_{9} d_{h} Y=Y \wedge Z, \quad-d \star_{9} Y=\frac{1}{2} Z \wedge Z,
$$

where $\star_{9}$ is the Hodge star operation on $\mathcal{S}$. The spacetime volume form has been decomposed as $\epsilon_{11}=\mathbf{e}^{+} \wedge \mathbf{e}^{-} \wedge \epsilon_{9}$, where $\epsilon_{9}$ is the volume form of $\mathcal{S}$. Similarly, the independent Einstein equations are

$$
\tilde{R}_{i j}+\tilde{\nabla}_{(i} h_{j)}-\frac{1}{2} h_{i} h_{j}=-\frac{1}{2} Y_{i j}^{2}+\frac{1}{12} Z_{i j}^{2}+\delta_{i j}\left(\frac{1}{12} Y^{2}-\frac{1}{144} Z^{2}\right),
$$

and

$$
\tilde{\nabla}^{i} h_{i}=2 \Delta+h^{2}-\frac{1}{3} Y^{2}-\frac{1}{72} Z^{2},
$$

where $\tilde{\nabla}$ and $\tilde{R}_{i j}$ are the Levi-Civita connection and the Ricci tensor of $\mathcal{S}$, respectively. For the rest of the notation see Appendix A.

An outline of the proof of the first part of the horizon conjecture is as follows. First one integrates the $d=11$ supergravity KSE along the coordinates $r, u$ which appear explicitly in the expressions for the fields in (349) and determines the remaining independent KSEs. Typically these are parallel transport equations acting on spinors that depend on the fluxes and can be thought of as a restriction of the gravitino KSE of the theory on $\mathcal{S}$. From these, one can define certain Dirac like operators, the horizon Dirac operators. A key next step is the proof of two Lichnerowicz type theorems which relate the zero modes of the horizon Dirac operators to the Killing spinors on $\mathcal{S}$. Then the index theorem is used to count the number of Killing spinors and establish the formula (348).

For the second part of the conjecture, one shows that for horizons with non-trivial fluxes and $N_{-} \neq 0$ there is always a pair of Killing spinors whose three vector bilinears are Killing and their Lie bracket algebra is $\mathfrak{s}(2$, $\mathbb{R})$. This establishes the horizon conjecture for $d=11$ supergravity.

\subsubsection{KSEs on the spatial horizon section}

The KSE of $d=11$ supergravity (275) can be integrated along the $u, r$ coordinates of the near horizon geometry to yield

$$
\epsilon=\phi_{+}+u \Gamma_{+} \Theta_{-} \phi_{-}+\phi_{-}+r \Gamma_{-} \Theta_{+}\left(\phi_{+}+u \Gamma_{+} \Theta_{-} \phi_{-}\right),
$$

where the spinors $\phi_{ \pm}$satisfy $\Gamma_{ \pm} \phi_{ \pm}=0$, depend only of the coordinates of $\mathcal{S}, \phi_{ \pm}=\phi_{ \pm}(y)$, and

$$
\Theta_{ \pm}=\frac{1}{4} h+\frac{1}{288} \not \pm \frac{1}{12} \chi .
$$

See Appendix A for the notation. Substituting (354) back into of the KSE (275) leads to a plethora of additional equations on $\phi_{ \pm}$. These include the conditions

$$
\left(\frac{1}{2} \Delta+2\left(\frac{1}{4} h-\frac{1}{288} \not+\frac{1}{12} Y\right) \Theta_{+}\right) \phi_{+}=0
$$

and

$$
\left(-\frac{1}{2} \Delta+2\left(-\frac{1}{4} h+\frac{1}{288} \not Z+\frac{1}{12} Y\right) \Theta_{-}\right) \phi_{-}=0,
$$

which will be used later in the investigation of warped AdS backgrounds. However after some involved analysis described in [221], which makes an essential use of the field equations and Bianchi identities in (351)-(353), one finds that the remaining independent KSEs are

$$
\nabla_{i}^{( \pm)} \phi_{ \pm} \equiv \tilde{\nabla}_{i} \phi_{ \pm}+\Psi_{i}^{( \pm)} \phi_{ \pm}=0
$$

where

$$
\Psi_{i}^{( \pm)}=\mp \frac{1}{4} h_{i}-\frac{1}{288} \not / Z_{i}+\frac{1}{36} Z_{i} \pm \frac{1}{24} \not / Y_{i} \mp \frac{1}{6} Y_{i} .
$$

These can be thought of as suitable restrictions of the gravitino $\operatorname{KSE}(275)$ on the spatial horizon section $\mathcal{S}$. Because of this we also refer to $\phi_{ \pm}$as Killing spinors on $\mathcal{S}$. In addition, it turns out that if $\phi_{-}$is a Killing spinor, $\nabla_{i}^{(-)} \phi_{-}=0$, then $\phi_{+}^{\prime}=\Gamma_{+} \Theta_{-} \phi_{-}$ will also be a Killing spinor, i.e.

$$
\nabla_{i}^{(+)} \phi_{+}^{\prime}=0 \text {. }
$$

This is the first indication that there may be a doubling in the number of Killing spinors for near horizon spacetimes. 


\subsubsection{Lichnerowicz type theorems}

To continue with the proof of the formula (348), the Killing spinors $\phi_{ \pm}$are related to the zero modes of Dirac like operators on $\mathcal{S}$. This is done via a Lichnerowicz type theorem. As a reminder, the classic Lichnerowicz theorem is as follows. Suppose that $\mathcal{D}$ is the Dirac operator on a Riemannian manifold $W$ which is compact without boundary. It can be established that $\mathcal{D}^{2}=\nabla^{2}-(1 / 4) R$, where $\nabla$ is the Levi-Civita connection of $W$ and $R$ its Ricci scalar. After a partial integration, one has that

$$
\int_{W}\|\mathcal{D} \eta\|^{2}=\int_{W}\|\nabla \eta\|^{2}+\frac{1}{4} \int_{W} R\|\eta\|^{2},
$$

where all the inner-products are positive definite and $\eta$ is a spinor. Clearly, if $R=0$, then all the zero modes of the Dirac operator are parallel and vice-versa.

Returning to the near horizon geometries, define the "horizon Dirac operators" as

$$
\mathcal{D}^{( \pm)} \phi_{ \pm}:=\Gamma^{i} \nabla_{i}^{( \pm)} \phi_{ \pm}=\Gamma^{i} \tilde{\nabla}_{i} \phi_{ \pm}+\Psi^{( \pm)} \phi_{ \pm},
$$

where

$$
\Psi^{( \pm)}=\Gamma^{i} \Psi_{i}^{( \pm)}=\mp \frac{1}{4} h h+\frac{1}{96} \not+\frac{1}{8} Y .
$$

Clearly, if $\nabla_{i}^{( \pm)} \phi_{ \pm}=0$, then $\mathcal{D}^{( \pm)} \phi_{ \pm}=0$. The converse is also true, i.e.

$$
\nabla_{i}^{( \pm)} \phi_{ \pm}=0 \Longleftrightarrow \mathcal{D}^{( \pm)} \phi_{ \pm}=0 \text {. }
$$

The proof of this for the $\mathcal{D}^{(+)}$operator relies on the use of the Hopf maximum principle. Using the field equations and Bianchi identities (351)-(353) and assuming that $\mathcal{D}^{(+)} \phi_{+}=0$, one can establish that

$$
\tilde{\nabla}^{i} \tilde{\nabla}_{i}\left\|\phi_{+}\right\|^{2}-h^{i} \tilde{\nabla}_{i}\left\|\phi_{+}\right\|^{2}=2\left\langle\nabla^{(+) i} \phi_{+}, \nabla_{i}^{(+)} \phi_{+}\right\rangle,
$$

where $\langle\cdot, \cdot\rangle$ is the $\operatorname{Spin}(9)$ invariant Hermitian inner product, see Appendix B. As $\langle\cdot, \cdot\rangle$ is positive definite, the right-hand-side of the equation above is positive semi-definite. On the other hand, $\left\|\phi_{+}\right\|^{2}$ as a function on the compact manifold $\mathcal{S}$ has a global maximum and a global minimum. These are critical points and the hessian is either negative or positive definite, respectively. Therefore, the left-hand-side of (365) changes sign while the right-hand-side is definite. So consistency requires that both sides must vanish establishing (364) for the $\mathcal{D}^{(+)}$operator and

$$
\left\|\phi_{+}\right\|^{2}=\text { const . }
$$

The constancy of the length of $\phi_{+}$will later be used in the investigation of the $\mathfrak{s}(2, \mathbb{R})$ symmetry.

The proof of (364) for the $\mathcal{D}^{(-)}$operator uses a partial integration argument similar to that of the standard Lichnerowicz theorem stated in the beginning of the section. In particular after imposing the field equations and Bianchi identities (351)(353), one can establish that

$$
\int_{\mathcal{S}}\left\|\mathcal{D}^{(-)} \phi_{-}\right\|^{2}=\int_{\mathcal{S}}\left\|\nabla^{(-)} \phi_{-}\right\|^{2}+\int_{\mathcal{S}}\left\langle\mathcal{C} \phi_{-}, \mathcal{D}^{(-)} \phi_{-}\right\rangle,
$$

where $\mathcal{C}$ is a Clifford algebra element that depends on the fluxes. As $\langle\cdot, \cdot\rangle$ is positive definite, if $\phi_{-}$is a zero mode of $\mathcal{D}^{(-)}$, then it will satisfy $\nabla^{(-)} \phi_{-}=0$ which proves the statement.

\subsubsection{Counting the killing spinors}

After the proof of the Lichnerowicz type theorems in the previous section, the apparatus to prove the first part of the horizon conjecture is in place. As $\mathcal{S}$ is an odd-dimensional manifold, the index of the Dirac operator vanishes and therefore (348) gives $N=2 N_{-}$.

To demonstrate this, the spacetime spin bundle $S$ restricted on $\mathcal{S}$ splits as $S=S_{+} \oplus S_{-}$, where the sections of $S_{ \pm}$are $\phi_{ \pm}, \phi_{ \pm} \in \Gamma\left(S_{ \pm}\right)$. Note that $S_{+}$and $S_{-}$are isomorphic as $\operatorname{Spin}(9)$ bundles. Observe that the horizon Dirac operator acts as $\mathcal{D}^{(+)}: \Gamma\left(S_{+}\right) \rightarrow \Gamma\left(S_{+}\right)$and similarly its adjoint $\left(\mathcal{D}^{(+)}\right)^{\dagger}: \Gamma\left(S_{+}\right) \rightarrow \Gamma\left(S_{+}\right)$, where the adjoint has been taken with respect to the $\operatorname{Spin}(9)$ invariant inner product $\langle\cdot, \cdot\rangle$, see Appendix B.

The horizon Dirac operator $\mathcal{D}^{(+)}$has the same principal symbol as the standard Dirac operator $\mathcal{D}$ and so $\operatorname{Index}\left(\mathcal{D}^{(+)}\right)=$ Index $(\mathcal{D})=0$ as the index of $\mathcal{D}$ vanishes. Thus

$$
\operatorname{dim} \operatorname{ker} \mathcal{D}^{(+)}=\operatorname{dim} \operatorname{ker}\left(\mathcal{D}^{(+)}\right)^{\dagger} .
$$

On the other hand, $\left(\mathcal{D}^{(+)}\right)^{\dagger} \Gamma_{+}=\Gamma_{+} \mathcal{D}^{(-)}$, and so

$$
\operatorname{dim} \operatorname{ker}\left(\mathcal{D}^{(+)}\right)^{\dagger}=\operatorname{dim} \operatorname{ker} \mathcal{D}^{(-)} .
$$

Therefore, one establishes that

$$
\operatorname{dim} \operatorname{ker} \mathcal{D}^{(+)}=\operatorname{dim} \operatorname{ker} \mathcal{D}^{(-)} .
$$


The number of supersymmetries of a near horizon geometry is the number of $\nabla^{( \pm)}$parallel spinors and so from the Lichnerowicz type theorems and the index argument above

$$
N=\operatorname{dim} \operatorname{ker} \mathcal{D}^{(+)}+\operatorname{dim} \operatorname{ker} \mathcal{D}^{(-)}=2 \operatorname{dim} \operatorname{ker} \mathcal{D}^{(-)}=2 N_{-} .
$$

This proves that the number of supersymmetries preserved by M-horizon geometries is even confirming the first part of the horizon conjecture for $d=11$ supergravity.

\subsubsection{Emergence of conformal symmetry}

The main task is to select two spacetime Killing spinors and demonstrate that the associated vector bilinears satisfy an $\mathfrak{s l}(2, \mathbb{R})$ Lie bracket algebra. As the near horizon geometries under investigation preserve some supersymmetry, $N=2 N_{-} \neq$ 0 , there is a $\phi_{-}$Killing spinor. As a consequence of (360), $\phi_{+}=\Gamma_{+} \Theta_{-} \phi_{-}$will also be a Killing spinor. The existence of both $\phi_{+}$and $\phi_{-}$suffices to construct two spacetime Killing spinors provided that $\phi_{+} \neq 0$, i.e. $\phi_{-}$is not in the kernel of $\Theta_{-}$. Indeed one can show that

$$
\operatorname{Ker} \Theta_{-} \neq\{0\} \Longleftrightarrow F=0, h=\Delta=0 .
$$

So if $\operatorname{Ker} \Theta_{-} \neq\{0\}$, the fluxes will vanish and therefore the near horizon geometries will be products $\mathbb{R}^{1,1} \times S^{1} \times N^{8}$, where $N^{8}$ has holonomy contained in $\operatorname{Spin}(7)$.

To sketch the proof of (372) assume that the kernel of $\Theta_{-}$is non-trivial and hence there is a $\phi_{-} \neq 0$ such that $\Theta_{-} \phi_{-}=0$. Taking the inner product of $\Theta_{-} \phi_{-}=0$ with $\phi_{-}$, one finds that $\Delta=0$ as $\left\|\phi_{-}\right\|$is no-where vanishing. Using the maximum principle and a partial integration argument, one can similarly proceed to prove (372). The details of the proof can be found in [221].

Therefore for horizons with non-trivial fluxes, for every $\phi_{-}$Killing spinor there is an associated non-trivial Killing spinor $\phi_{+}=\Gamma_{+} \Theta_{-} \phi_{-}$. In turn, the near horizon spacetime admits two Killing spinors given by

$$
\begin{aligned}
& \epsilon_{1}=\epsilon\left(\phi_{-}, 0\right)=\phi_{-}+u \phi_{+}+r u \Gamma_{-} \Theta_{+} \phi_{+}, \\
& \epsilon_{2}=\epsilon\left(\phi_{-}, \phi_{+}\right)=\phi_{+}+r \Gamma_{-} \Theta_{+} \phi_{+}, \quad \phi_{+}=\Gamma_{+} \Theta_{-} \phi_{-} .
\end{aligned}
$$

These give rise to 3 Killing vector bi-linears, see also (301), given by

$$
\begin{aligned}
X_{1}:=\left\langle\left(\Gamma_{+}-\Gamma_{-}\right) \epsilon_{1}, \Gamma^{A} \epsilon_{2}\right\rangle \partial_{A}= & -2 u\left\|\phi_{+}\right\|^{2} \partial_{u}+2 r\left\|\phi_{+}\right\|^{2} \partial_{r}+V^{i} \tilde{\partial}_{i}, \\
X_{2}:=\left\langle\left(\Gamma_{+}-\Gamma_{-}\right) \epsilon_{2}, \Gamma^{A} \epsilon_{2}\right\rangle \partial_{A}= & -2\left\|\phi_{+}\right\|^{2} \partial_{u}, \\
X_{3}:=\left\langle\left(\Gamma_{+}-\Gamma_{-}\right) \epsilon_{1}, \Gamma^{A} \epsilon_{1}\right\rangle \partial_{A}= & -2 u^{2}\left\|\phi_{+}\right\|^{2} \partial_{u}+\left(2\left\|\phi_{-}\right\|^{2}\right. \\
& \left.+4 r u\left\|\phi_{+}\right\|^{2}\right) \partial_{r}+2 u V^{i} \tilde{\partial}_{i},
\end{aligned}
$$

where $V=\left\langle\Gamma_{+} \phi_{-}, \Gamma^{i} \phi_{+}\right\rangle \tilde{\partial}_{i}$ is a Killing vector on $\mathcal{S}$ which leaves all the data invariant and $\tilde{\partial}_{i}=e_{i}^{I} \partial_{I}$. To simplify somewhat the expressions for the Killing vector fields above, we have used that

$$
-\Delta\left\|\phi_{+}\right\|^{2}+4\left\|\Theta_{+} \phi_{+}\right\|^{2}=0,\left\langle\phi_{+}, \Gamma_{i} \Theta_{+} \phi_{+}\right\rangle=0 .
$$

These follow either from the KSEs or equivalently from the Killing condition on $X_{1}, X_{2}$ and $X_{3}$.

A straightforward computation reveals that the Lie bracket algebra of $X_{1}, X_{2}$ and $X_{3}$ is $\mathfrak{s l}(2, \mathbb{R})$,

$$
\begin{aligned}
& {\left[X_{1}, X_{2}\right]=2\left\|\phi_{+}\right\|^{2} X_{2}, \quad\left[X_{2}, X_{3}\right]=-4\left\|\phi_{+}\right\|^{2} X_{1},} \\
& {\left[X_{3}, X_{1}\right]=2\left\|\phi_{+}\right\|^{2} X_{3},}
\end{aligned}
$$

where $\left\|\phi_{+}\right\|$is constant, see (366). Note that the emergence of the $\mathfrak{s l}(2, \mathbb{R})$ symmetry is dynamical as the proof of its existence requires the use of the field equations. This completes the proof of the second part of the horizon conjecture.

A special case arises whenever $V=0$. This together with the Killing condition of $X_{1}, X_{2}$ and $X_{3}$ implies that $h=\Delta^{-1} d \Delta$, see [221] for the proof. The spacetime is a warped product of $A d S_{2}$ with the horizon section $\mathcal{S}, A d S_{2} \times_{w} \mathcal{S}$. Therefore, the warped $A d S_{2}$ solutions of supergravity theories are included in the near horizon geometries and therefore all the properties proven for the latter also hold for the former.

As has already been mentioned, the horizon conjecture demonstrated for $d=11$ supergravity also holds for other theories including (massive) IIA, IIB, $d=5$ and $d=4$ supergravities. In particular, one can show after integrating over the $r, u$ coordinates that the remaining independent KSEs are those naively expected from restricting the gravitino and algebraic KSEs on the spatial horizon section $\mathcal{S}$. The form of the Killing spinors is exactly as in (354) though of course the field content of $\Theta_{ \pm}$is different. One of the additional complications that arises in the proof of Lichnerowicz type theorems is the presence of the algebraic KSEs, like for example a dilatino and/or a gaugino KSE. Nevertheless after an appropriate choice of Dirac horizon operators, it is possible to prove with the use of maximum principle and partial integration arguments that the zero modes of the horizon Dirac operators are Killing, i.e. they solve both the gravitino and algebraic KSEs. The $\mathfrak{s}(2, \mathbb{R})$ conformal symmetry emerges in the same way as described for $d=11$ supergravity. 


\section{AdS and Minkowski flux compactifications}

Amongst the $d=10$ and $d=11$ supersymmetric solutions which have found widespread applications in supergravity, string, and M-theory compactifications and in the AdS/CFT correspondence are warped products of Minkowski and AdS spaces with some internal space, for reviews see e.g. [229,230] and [231]. Such backgrounds are characterized by the requirement that they are invariant under the isometry group of either the AdS or Minkowski subspaces. Many of the properties of these backgrounds can be investigated in a unified way irrespective on whether they are solutions of $d=11$, (massive) IIA or IIB supergravities. These properties include the counting of the number of preserved supersymmetries, as well as the Killing superalgebras. However to be concrete, we shall mostly present the analysis for the $d=11$ backgrounds and only comment on the results for other theories.

\subsection{Warped AdS and Minkowski backgrounds from horizons}

Warped AdS and Minkowski backgrounds are examples of near horizon geometries possibly allowing for non-compact spatial horizon sections [232]. To see this, consider the metric

$$
d s^{2}=A^{2} d s^{2}\left(A d S_{n}\right)+d s^{2}\left(N^{d-n}\right),
$$

on a warped product of $A d S_{n}$ with an internal space $N^{d-n}, A d S_{n} \times{ }_{w} N^{d-n}$, where $A$ is the warp factor which depends only on the coordinates of $N^{d-n}$ and

$$
\begin{aligned}
& d s^{2}\left(A d S_{n}\right)=e^{\frac{2 z}{\ell}}\left(2 d u d v+\sum_{a=1}^{n-3}\left(d x^{a}\right)^{2}\right)+d z^{2}, \quad n>2, \\
& d s^{2}\left(A d S_{2}\right)=2 d u\left(d v-\ell^{-2} v^{2} d u\right), \quad n=2,
\end{aligned}
$$

is the metric on the Poincare patch of $A d S_{n}$ space. The parameter $\ell$ is the radius of $A d S_{n}$.

To see that the metrics above can be written in a near horizon form (347), for $n>2$ perform the coordinate transformation

$$
v=A^{-2} e^{-\frac{2 z}{\ell}} r,
$$

with the rest of the coordinates unchanged, to find that the metric transforms to

$$
\begin{aligned}
d s^{2}= & 2 d u\left(d r-r\left(\frac{2}{\ell} d z+d \log A^{2}\right)\right)+A^{2}\left(d z^{2}+e^{\frac{2 z}{\ell}} \sum_{a=1}^{n-3}\left(d x^{a}\right)^{2}\right) \\
& +d s^{2}\left(N^{d-n}\right) .
\end{aligned}
$$

This is a near horizon metric (347) with $\Delta=0, h=-\left(\frac{2}{\ell} d z+d \log A^{2}\right)$ and metric on the spatial horizon section

$$
d s^{2}(\mathcal{S})=A^{2}\left(d z^{2}+e^{\frac{2 z}{\ell}} \sum_{a=1}^{n-3}\left(d x^{a}\right)^{2}\right)+d s^{2}\left(N^{d-n}\right) .
$$

The spatial horizon section is the warped product of the hyperbolic space $H^{n-2}$ with the internal space $N^{d-n}, \mathcal{S}=H^{n-2} \times{ }_{w}$ $N^{d-n}$.

Similarly for the warped $A d S_{2}$ backgrounds, $A d S_{2} \times{ }_{w} N^{d-n}$, perform the coordinate transformation $r=v A^{2}$ to find that the metric can be put into the near horizon form (347) with $\Delta=\ell^{-2} A^{-2}$ and $h=-d \log A^{2}$. The near horizon section $\mathcal{S}$ is identified with the internal space $N^{d-2}, \mathcal{S}=N^{d-2}$.

The rest of the form fluxes of the warped $A d S_{n}$ backgrounds of supergravity theories can also be put into a near horizon form. In particular a typical $k$-form flux field strength $F, k \geq n$, can be written as

$$
F=d \operatorname{vol}\left(A d S_{n}\right) \wedge W+Z=\mathbf{e}^{+} \wedge \mathbf{e}^{-} \wedge Y+Z,
$$

where $W$ and $Z$ are $(k-n)$ - and $k$-forms on $N^{d-n}$ which depend only on the coordinates of $N^{d-n}$. If $k<n$, then $F$ will be written as above with $W=0$. Note also that the terms $d_{h} Y$ in the fluxes, see (349), must vanish as they are not invariant under the isometries of $A d S_{n}$.

The warped Minkowski backgrounds can be viewed as a special case of AdS backgrounds which arise in the limit that the AdS radius $\ell$ goes to infinity, $\ell \rightarrow \infty$. In particular notice that in this limit the metric (380) on $A d S_{n} \times{ }_{w} N^{d-n}$ becomes a metric on $\mathbb{R}^{n-1,1} \times{ }_{w} N^{d-n}$. 


\subsection{Solution of KSEs for AdS backgrounds}

In the literature, the KSEs of supergravity theories have been solved for warped AdS backgrounds in many different ways. Some approaches involve a factorization of the spacetime Killing spinors into Killing spinors on the AdS subspace and those on the internal space. There is an extensive literature on AdS solutions and an incomplete list of works is [202,233-249]. Here we shall adopt the approach developed in [250-252] in which the spacetime KSEs are solved directly for these backgrounds without any assumptions. This utilizes all the technology that has been developed to solve the KSEs for near horizon geometries described in Section 11.3.2. Apart from generality, this method allows the analysis of some of the properties of the backgrounds simultaneously without reference to a particular AdS background or the theory that it is a solution of. A comparison of the different approaches can be found in [253].

To begin let us write the $d=11$ warped AdS backgrounds as

$$
\begin{gathered}
d s^{2}=2 \mathbf{e}^{-} \mathbf{e}^{+}+\left(\mathbf{e}^{z}\right)^{2}+\sum_{a=1}^{n-3}\left(\mathbf{e}^{a}\right)^{2}+d s^{2}\left(N^{11-n}\right), \\
F=d \operatorname{vol}\left(A d S_{n}\right) \wedge W+Z=\mathbf{e}^{+} \wedge \mathbf{e}^{-} \wedge Y+Z,
\end{gathered}
$$

where $W$ and $X$ are $(4-n)$ - and 4-forms on $N^{11-n}$ and $Y$ has been introduced to facilitate progress in the analysis that follows. The spacetime coframe is chosen as

$$
\begin{aligned}
& \mathbf{e}^{-}=d u, \quad \mathbf{e}^{+}=d r-\left(\frac{2}{\ell} d z+d \log A^{2}\right), \quad \mathbf{e}^{z}=A d z, \\
& \mathbf{e}^{a}=A e^{\frac{z}{\ell}} d x^{a}, \quad \mathbf{e}^{i}=\mathbf{e}_{l}^{i} d y^{I},
\end{aligned}
$$

the 2 -form $Y$ is

$$
\begin{aligned}
& Y=\mathbf{e}^{z} \wedge W,(n=3) ; Y=W \mathbf{e}^{z} \wedge \mathbf{e}^{1},(n=4) ; \\
& Y=0, n>4,
\end{aligned}
$$

and $d s^{2}\left(N^{11-n}\right)=\delta_{i j} \mathbf{e}^{i} \mathbf{e}^{j}$.

The field equations of $d=11$ supergravity can be rewritten in terms of the component fields of the $A d S_{n}$ backgrounds as given in (383). In particular, the field equation for the warp factor, which arises from the Einstein equations along the $A d S_{n}$ subspace, is

$$
D^{2} \log A=-\frac{n-1}{\ell^{2} A^{2}}-n \partial^{i} \log A \partial_{i} \log A+\frac{1}{6} Y^{2}+\frac{1}{144} Z^{2},
$$

where $D$ is the Levi-Civita connection of the internal space $N^{11-n}$. It can be argued that for smooth solutions $A$ is no-where zero. The rest of the field equations can be found in [250] and they will not be repeated here.

The integration of the $d=11$ gravitino KSE (275) over the $A d S_{n}$ subspace of $A d S_{n} \times N^{d-n}$ leads to an expression for the Killing spinor which is explicit in AdS coordinates. The remaining KSEs include a restriction of (275) to the internal space $N^{11-n}$. In addition to these, there are also new algebraic conditions on the spinors which arise as integrability conditions of the integration of (275) over $A d S_{n}$. In particular, a spacetime Killing spinor, $\epsilon$, can be written as

$$
\epsilon=\epsilon_{1}+\epsilon_{2}+\epsilon_{3}+\epsilon_{4},
$$

where

$$
\begin{aligned}
& \epsilon_{1}=\sigma_{+}, \quad \epsilon_{2}=\sigma_{-}-\ell^{-1} e^{\frac{z}{\ell}} x^{a} \Gamma_{a z} \sigma_{-}-\ell^{-1} A^{-1} u \Gamma_{+z} \sigma_{-}, \\
& \epsilon_{3}=e^{-\frac{z}{\ell}} \tau_{+}-\ell^{-1} A^{-1} r e^{-\frac{z}{\ell}} \Gamma_{-z} \tau_{+}-\ell^{-1} x^{a} \Gamma_{a z} \tau_{+}, \quad \epsilon_{4}=e^{\frac{z}{\ell}} \tau_{-},
\end{aligned}
$$

and all the gamma matrices are in the coframe basis (384). The $\sigma_{ \pm}$and $\tau_{ \pm}$spinors satisfy the lightcone projections $\Gamma_{ \pm} \sigma_{ \pm}=$ $\Gamma_{ \pm} \tau_{ \pm}=0$, and depend only on the coordinates of $N^{11-n}$. Furthermore, $\sigma_{ \pm}$and $\tau_{ \pm}$are parallel along the internal space $N^{11-n}$

$$
D_{i}^{( \pm)} \sigma_{ \pm}=0, \quad D_{i}^{( \pm)} \tau_{ \pm}=0,
$$

and satisfy the algebraic conditions

$$
\Xi^{( \pm)} \sigma_{ \pm}=0, \quad \Xi^{( \pm)} \tau_{ \pm}=\mp \frac{1}{\ell A} \tau_{ \pm},
$$

where

$$
\begin{aligned}
D_{i}^{( \pm)} & :=D_{i} \pm \frac{1}{2} \partial_{i} \log A-\frac{1}{288} \not / Z_{i}+\frac{1}{36} Z_{i} \pm \frac{1}{24} \not / Y_{i} \mp \frac{1}{6} Y_{i}, \\
\Xi^{( \pm)} & :=-\frac{1}{2} \Gamma_{z} \not \partial \log A \mp \frac{1}{2 \ell A}+\frac{1}{288} \Gamma_{Z} \not \subset \pm \frac{1}{6} \not \ell,
\end{aligned}
$$


and where $Q$ is defined by the relation $Y=\mathbf{e}^{z} \wedge Q$. Note that the last term in the first equation vanishes for $n=4$ as $i_{X} Y=0$ for any vector field $X$ on the internal space.

The conditions (389) on $\sigma_{ \pm}$and $\tau_{ \pm}$are those that are thought of as restrictions of the spacetime gravitino KSE to the internal space. While those in (390) are the new ones that arise as integrability conditions. We shall refer to both as the remaining KSEs.

An outline of the proof of the formulae (387) and (388) for the Killing spinor $\epsilon$ is as follows. As the warped AdS backgrounds can be put in a near horizon form, one can integrate along the $u, r$ coordinates to get the expression of the Killing spinor as for near horizon geometries in (354). Then the independent KSEs (358) on $\phi_{ \pm}$evaluated along the $z$ coordinate can be written as

$$
\partial_{z} \phi_{ \pm}=A \Xi^{( \pm)} \phi_{ \pm}
$$

where $\Xi^{( \pm)}$is as in (390). Taking another $z$ derivative of the above equation and using the conditions (356) and (357), one finds that

$$
\partial_{z}^{2} \phi_{ \pm} \pm \frac{1}{\ell} \partial_{z} \phi_{ \pm}=0
$$

Therefore, the solutions are $\phi_{ \pm}=\kappa_{ \pm}+e^{\mp \frac{z}{\ell}} \lambda_{ \pm}$, where $\kappa_{ \pm}$and $\lambda_{ \pm}$are independent of the $u, r, z$ coordinates. Substituting $\phi_{ \pm}$ back into (392), one finds the algebraic conditions in (390) on $\kappa_{ \pm}$and $\lambda_{ \pm}$, respectively. The integration over the remaining $x^{a}$ AdS coordinates does not produce additional integrability conditions. Performing the integration over the $x^{a}$ coordinates and using the algebraic KSEs (390), one finds the expression for the Killing spinors as in (387) and (388).

The solution of the KSEs of $d=10$ supergravities for warped AdS backgrounds proceeds as for the $d=11$ ones described above. The expression for the Killing spinors is the same as (387) and (388). The spinors $\sigma_{ \pm}$and $\tau_{ \pm}$satisfy some remaining KSEs. These include a restriction of the original KSEs of these theories on the internal spaces $N^{10-n}$, like (389), and some additional ones that arise as integrability conditions of the integration of the gravitino KSE over $A d S_{n}$, like (390). Of course the former, apart from parallel transport equations, like those of (389) associated with the gravitino KSE, include also algebraic KSEs which are restrictions of the algebraic KSE of these theories to $N^{10-n}$. For example in IIA supergravity $\tau_{ \pm}$and $\sigma_{ \pm}$satisfy a condition which is a restriction of dilatino KSE to the internal space.

\subsection{Counting supersymmetries for warped AdS backgrounds}

The number of supersymmetries preserved by warped AdS backgrounds come with multiplicities. This is because Clifford algebra operations act on the solutions of the remaining KSEs (389) and (390) generating new ones. In particular, if $\sigma_{ \pm}$are solutions of (389) and (390), then

$$
\tau_{ \pm}:=\Gamma_{z a} \sigma_{ \pm}, \forall a,
$$

will also be solutions. As the Clifford algebra operations $\Gamma_{z a}$ are invertible, the converse is also true.

Similarly, if $\sigma_{+}$and $\tau_{+}$are solutions, then

$$
\sigma_{-}:=A \Gamma_{-} \Gamma_{z} \sigma_{+}, \quad \tau_{-}:=A \Gamma_{-} \Gamma_{z} \tau_{+},
$$

will also be solutions, and vice versa.

Furthermore, if $\sigma_{+}$is Killing spinor of (389) and (390), then

$$
\sigma_{+}^{\prime}:=\Gamma_{a b} \sigma_{+}, \quad \forall a, b, \text { with } a<b,
$$

will also be Killing spinors. Therefore, one can start from a solution and act with the Clifford algebra operations above to construct a whole multiplet.

The counting proceeds with the identification of the linearly independent solutions in each multiplet. The number of Killing spinors of an $A d S_{n}$ background is the number of Killing spinors in each multiplet times the number of multiplets that can occur. First, we have seen that warped $A d S_{2}$ backgrounds preserve an even number of supersymmetries as a special case of near horizon geometries. Since for $n \geq 3$, the $\sigma_{-}$and $\tau_{-}$solutions are generated from those of $\sigma_{+}$and $\tau_{+}$, it suffices to count the latter. $A d S_{3}$ backgrounds can admit either $\sigma_{+}$or $\tau_{+}$or both $\sigma_{+}$and $\tau_{+}$Killing spinors. Therefore the multiplet contains always the pair $\sigma_{ \pm}$or $\tau_{ \pm}$or both, and so these backgrounds preserve $2 k$ supersymmetries.

For $A d S_{n}, n>3$, the $\tau_{+}$solutions are generated from those of $\sigma_{+}$. Therefore the number of linearly independent Killing spinors in a multiplet is four times the linearly independent $\sigma_{+}$spinors that arise from the application of (396). For warped $A d S_{4}$ backgrounds, (396) does not produce any degeneracy and so the number of supersymmetries preserved are $4 \mathrm{k}$. For warped $A d S_{5}$ backgrounds, (396) yields two linearly independent $\sigma_{+}$spinors for each multiplet. Therefore these backgrounds preserve $8 k$ supersymmetries. A similar counting leads to the conclusion that warped $A d S_{6}$ backgrounds preserve $16 k$ supersymmetries. Note though that to correctly count the number of Killing spinors for warped $A d S_{7}$ backgrounds, the $\sigma_{+}$ Killing spinor which is used to construct a multiplet can be chosen to satisfy the condition $\Gamma_{1234} \sigma_{+}= \pm \sigma_{+}$. Such a choice leads to the possibility of warped $A d S_{7}$ backgrounds that can preserve 16 supersymmetries. These results are tabulated in Table 4. The counting of supersymmetries of warped AdS backgrounds in $d=10$ type II supergravities can be done in the same way leading to the same results as for $d=11$ supergravity. 
Table 4

The proof that warped $A d S_{2}$ backgrounds preserve $2 k$ supersymmetries requires that the fields are smooth and the internal space is compact without boundary. For the rest of $A d S_{n}$ and $\mathbb{R}^{n-1,1}$ backgrounds, no such assumptions are necessary. In all cases $N \leq 32$. This counting of supersymmetries applies to all $d=11$ and $d=10$ type II supergravities.

\begin{tabular}{lll}
\hline & $N$ for $A d S_{n}$ & $N$ for $\mathbb{R}^{n-1,1}$ \\
\hline$n=2$ & $2 k$ & - \\
$n=3$ & $2 k$ & $2 k$ \\
$n=4$ & $4 k$ & $4 k$ \\
$n=5$ & $8 k$ & $8 k$ \\
$n=6$ & $16 k$ & $8 k$ \\
$n=7$ & $16 k$ & $16 k$ \\
\hline
\end{tabular}

\subsection{KSEs and counting supersymmetries for warped Minkowski backgrounds}

To find the Killing spinors of warped Minkowski backgrounds, one follows the same steps as in the AdS case. It turns out that the Killing spinors can be written as

$$
\begin{aligned}
\epsilon=\sigma_{+} & +u \Gamma_{+} \Gamma_{z} \Xi^{(-)} \sigma_{-}+\sum_{m} x^{m} \Gamma_{m} A \Gamma_{z} \Xi^{(+)} \sigma_{+} \\
& +\sigma_{-}+r \Gamma_{-} \Gamma_{z} \Xi^{(+)} \sigma_{+}+\sum_{m} x^{m} \Gamma_{m} A \Gamma_{z} \Xi^{(-)} \sigma_{-},
\end{aligned}
$$

where the coordinates of the Minkowski space are $\left(u, r, x^{m}\right)=\left(u, r, z, x^{a}\right)$ and all the gamma matrices are in a coframe basis. The remaining KSEs are

$$
D_{i}^{( \pm)} \sigma_{ \pm}=0, \quad\left(\Xi^{( \pm)}\right)^{2} \sigma_{ \pm}=0,
$$

where $D_{i}^{( \pm)}$is given in (391) and

$$
\Xi^{( \pm)}:=-\frac{1}{2} \Gamma_{z} \not \log A+\frac{1}{288} \Gamma_{z} \not \pm \frac{1}{6} \not .
$$

Before we describe the proof of this observe that the Killing spinors may depend on the coordinates of the Minkowski space provided that $\sigma_{ \pm} \notin \operatorname{ker} \Xi^{( \pm)}$. This may seem a bit puzzling but it should be allowed. This is because $A d S_{n}$ spaces in the Poincaré patch can be viewed as warped Minkowski $\mathbb{R}^{n-2,1}$ backgrounds, $A d S_{n}=\mathbb{R}^{n-2,1} \times \times_{w}$, and we have demonstrated that Killing spinors of AdS spaces (387) and (388) depend on all AdS coordinates including those of the $\mathbb{R}^{n-2,1}$ subspace. For more discussion on this see [253].

Returning to the proof of (397), one first solves the gravitino KSE along the coordinates ( $u, r$ ), as for near horizon geometries, to yield the expression of the Killing spinor $\epsilon$ in terms of the $(u, r)$ coordinates and in terms of the $\phi_{ \pm}$spinors as for near horizon geometries (354). Then the gravitino KSE (358) on $\phi_{ \pm}$along the $z$ coordinate reads

$$
\partial_{z} \phi_{ \pm}=A \Xi^{( \pm)} \phi_{ \pm}
$$

where $\Xi^{( \pm)}$is as in (399). Clearly a solution of this is $\phi_{ \pm}=\kappa_{ \pm}+z A \Xi^{( \pm)} \kappa_{ \pm}$provided that $\left(\Xi^{( \pm)}\right)^{2} \kappa_{ \pm}=0$, where $\kappa_{ \pm}$does not depend on $z$. Proceeding in a similar way and solving the gravitino KSE along the remaining coordinates of the Minkowski subspace, one finds (397) and (398).

To count the multiplicities of Killing spinors observe that if $\sigma_{-}$is a Killing spinor, then $\sigma_{+}=A^{-1} \Gamma_{+} \Gamma_{m} \sigma_{-}$will also be Killing spinors for every $m$, and vice versa. Furthermore, if $\sigma_{+}$is a Killing spinor, then $\sigma_{+}^{\prime}=\Gamma_{m n} \sigma_{+}$will also be Killing spinors for every $m<n$. Counting the independent Killing spinors in a way similar to that presented for warped AdS backgrounds, one establishes the results of Table 4 . Notice that the counting of supersymmetries of warped $\mathbb{R}^{1,1}$ backgrounds has been excluded from the results in Table 4 . This is because warped $\mathbb{R}^{1,1}$ backgrounds with fluxes may either be singular or the internal space may not compact. Therefore, the counting of supersymmetries presented for the warped $A d S_{2}$ backgrounds cannot straightforwardly be adapted to this case.

\subsection{A non-existence theorem for smooth warped de-Sitter and Minkowski compactifications}

There are restrictions on the existence of smooth warped de-Sitter and Minkowski supergravity compactifications [158, 159]. To see this consider a $d=10$ or a $d=11$ supergravity theory and seek warped flux compactification solutions with metric

$$
d s^{2}=e^{2 \phi} d s^{2}\left(M^{n}\right)+d s^{2}\left(N^{D-n}\right),
$$

where $e^{2 \phi}$ is the warp factor, $M^{n}$ is either Minkowski, $\mathbb{R}^{n-1,1}$, de-Sitter, $d S_{n}$, or anti-de-Sitter, $A d S_{n}$, space, and $N^{d-n}$ is an internal space. The rest of the fields are non-vanishing but they are suppressed in the statements that follow.

The Einstein field equations along $M^{n}$, or equivalently the field equation of the warp factor, can be written as

$$
D^{2} e^{n \phi}=q e^{(n-2) \phi} R\left(M^{n}\right)+e^{n \phi} S(F),
$$


where $q \in \mathbb{R}_{>0}, R\left(M^{n}\right)$ is the scalar curvature of $M^{n}, D$ is the Levi-Civita connection of the internal space $N^{d-n}$ and $S(F)$ a function that depends on the other fields of the theory. See also the warp factor field equation for $A d S_{n}$ backgrounds in (386). Clearly, $S(F)$ depends on both the choice of background and the theory under investigation. But the key observation is that for $d=11$, (massive) IIA and IIB supergravities $S(F) \geq 0$ and vanishes whenever the fields are zero.

As for de-Sitter backgrounds, $R\left(d S_{n}\right)>0$, an application of the Hopf maximum principle, or equivalently a partial integration argument, reveals that there are no smooth warped compactifications with compact, without boundary, internal space. Moreover there are no smooth warped Minkowski compactifications, $R\left(\mathbb{R}^{n-1,1}\right)=0$, with non-trivial fluxes and compact, without boundary, internal space. The only such smooth compactifications are those with trivial fluxes and with constant warp factor, e.g. Calabi-Yau type of compactifications. It is essential to stress that this argument does not depend on whether or not the backgrounds preserve some supersymmetry. It is solely based on the Einstein field equation and in particular the field equation of the warp factor. These non-existence theorems have consequences for the applications of supergravity and string theory compactifications in particle physics and cosmology, see e.g. [254] for a review and references therein. The former require flux compactifications for moduli stabilization while the latter rely on the existence of de-Sitter vacua.

\subsection{Killing superalgebras for warped AdS backgrounds}

To make further progress towards the investigation of the geometry of warped AdS backgrounds, one may proceed to find their Killing superalgebras, see Section 8.6. As we shall demonstrate later, these are sufficient to determine all AdS backgrounds which preserve $N>16$ supersymmetries in $d=11$ and type II $d=10$ supergravities.

Before we present the key steps of the proof identifying all Killing superalgebras of AdS backgrounds [255], let us state our assumptions, see also [256] for an early superalgebra computation. Take $\mathfrak{g}$ to be the Killing superalgebra of a warped $A d S_{n}$ background. The even part of the superalgebra $\mathfrak{g}_{0}$ contains the isometries $\mathfrak{s o}(n-1,2)$ of the $A d S_{n}$ subspace. This can be verified after an explicit computation of the vector bilinears of the Killing spinors (387). One may also expect that $\mathfrak{g}_{0}=\mathfrak{s o}(n-1,2) \oplus \mathfrak{t}_{0}$, $n \neq 3$, where $t_{0}$ is the Lie algebra of isometries of the internal space $N^{d-n}$. However this is not always the case. One way to see this is to observe that $A d S_{k}$ backgrounds can be written as warped products, $A d S_{k}=A d S_{n} \times{ }_{w} \mathbb{R}^{k-n}$, of $A d S_{n}$ spaces for $n<k$. The internal space $N^{d-n}$ of $A d S_{n}$ is $N^{d-n}=\mathbb{R}^{k-n} \times{ }_{w} N^{d-k}$, where $N^{d-k}$ is the internal space of the $A d S_{k}$ background. From the perspective of $A d S_{n}$, there exist Killing vector fields with components on the both $A d S_{n}$ and its internal space $N^{d-n}$ which cannot be separated into isometries of $A d S_{n}$ and isometries of $N^{d-n}$. This is because $\mathfrak{s o}(k-1,2)$ cannot be decomposed as $\mathfrak{s o}(k-1,2)=\mathfrak{s o}(n-1,2) \oplus \mathfrak{m}$, where $\mathfrak{m}$ is also a Lie algebra. To avoid such a phenomenon developing, we shall assume that either $\mathfrak{g}_{0}=\mathfrak{s o}(n-1,2) \oplus \mathfrak{t}_{0}$, where $\mathfrak{t}_{0}$ is the algebra of isometries of the internal space, or that the internal space of $A d S_{n}$ backgrounds is compact without boundary. In either case, one finds the following conditions on the bilinears

$$
\left\langle\sigma_{+}, \Gamma_{i} \Gamma_{a} \sigma_{+}^{\prime}\right\rangle=0,\left\langle\tau_{+}, \Gamma_{i} \Gamma_{z} \sigma_{+}\right\rangle=0,
$$

for every Killing spinor $\sigma_{+}, \sigma_{+}^{\prime}$ and $\tau_{+}$. Of course for $\operatorname{AdS}_{n}, n>3$, backgrounds the two conditions are equivalent while for $\mathrm{AdS}_{3}$ backgrounds only the latter applies. A consequence of the algebraic KSEs (390) is then that

$$
\left\langle\tau_{+}, \sigma_{+}\right\rangle=0 .
$$

Another consequence of the requirement that $\mathfrak{g}_{0}=\mathfrak{s o}(n-1,2) \oplus \mathfrak{t}_{0}$, and $\operatorname{so}\left[\mathfrak{s o}(n-1,2), \mathfrak{t}_{0}\right]=0$, and the Killing condition on the spacetime vector bilinears (301) of the Killing spinors (387) is that

$$
\tilde{X}^{i} \partial_{i} A=0,\left\langle\sigma_{+}, \sigma_{+}^{\prime}\right\rangle=\text { const },
$$

where $\tilde{X}=\left\langle\sigma_{+}, \Gamma^{i} \Gamma_{z} \sigma_{+}^{\prime}\right\rangle \tilde{\partial}_{i}$ are the Killing vector bilinears of the internal space. Therefore, the warp factor is invariant under the action of $\mathfrak{g}_{0}$. As the inner product of Killing spinors is constant from now on without loss of generality we shall set

$$
\left\langle\sigma_{+}^{r}, \sigma_{+}^{s}\right\rangle=\frac{1}{2} \delta^{r s}
$$

where for $A d S_{n}, n>3, r, s=1, \ldots, N / 4$, and for $A d S_{2}$ and $A d S_{3}, r, s=1, \ldots, N / 2$. The identification of Killing superalgebras is somewhat different for $A d S_{n}, n>3$ and $A d S_{n}, n \leq 3$ backgrounds and therefore they will treated differently.

\subsubsection{Killing superalgebras for $A d S_{n}, n>3$, backgrounds}

Supposing that the conditions on the bilinears (403) hold, one can demonstrate that the Killing superalgebras $g$ of warped $A d S_{n}, n>3$, backgrounds are those tabulated in Table 5 . Moreover the isometry algebras $t_{0}$ of their internal space are presented in Table 6.

The proof of these results relies on the fact that the dependence of the Killing spinors, (387) and (388), on the coordinates of the $A d S_{n}$ subspace of the warped backgrounds is known. As a result, one can explicitly compute the (anti-) commutators

$$
\begin{aligned}
& \left\{\mathfrak{g}_{1}, \mathfrak{g}_{1}\right\}=\mathfrak{s o}(n-1,2)+\mathfrak{t}_{0}, \\
& {\left[\mathfrak{s o}(n-1,2), \mathfrak{g}_{1}\right] \subseteq \mathfrak{g}_{1} .}
\end{aligned}
$$

The key commutator that needs to be found is $\left[\mathfrak{t}_{0}, \mathfrak{g}_{1}\right]$. This typically requires some information on the underlying geometry of the internal space $N^{d-n}$. However for $A d S_{n}, n>3$, backgrounds this is not necessary and the result follows as a consequence 
Table 5

$f^{*}(4)$ is a different real form of $\mathfrak{f}(4)$, which appears in the $\mathrm{AdS}_{3}$ case.

\begin{tabular}{|c|c|c|c|c|}
\hline \multicolumn{5}{|c|}{ Killing superalgebras of $d=10,11$ warped $\operatorname{AdS}_{n}, n>3$, solutions } \\
\hline$N$ & $\mathrm{AdS}_{4}$ & $\mathrm{AdS}_{5}$ & $\mathrm{AdS}_{6}$ & $\mathrm{AdS}_{7}$ \\
\hline 4 & $\mathfrak{o s p}(1 \mid 4)$ & - & - & - \\
\hline 8 & $\mathfrak{o s p}(2 \mid 4)$ & $\mathfrak{s l}(1 \mid 4)$ & - & - \\
\hline 12 & $\mathfrak{o s p}(3 \mid 4)$ & - & - & - \\
\hline 16 & $\mathfrak{o s p}(4 \mid 4)$ & $\mathfrak{s l}(2 \mid 4)$ & $f^{*}(4)$ & $\mathfrak{o s p}(6,2 \mid 2)$ \\
\hline 20 & $\mathfrak{o s p}(5 \mid 4)$ & - & - & - \\
\hline 24 & $\mathfrak{o s p}(6 \mid 4)$ & $\mathfrak{s l}(3 \mid 4)$ & - & - \\
\hline 28 & $\mathfrak{o s p}(7 \mid 4)$ & - & - & - \\
\hline 32 & $\mathfrak{o s p}(8 \mid 4)$ & $\mathfrak{s l}(4 \mid 4) / 1_{8 \times 8}$ & - & $\mathfrak{o s p}(6,2 \mid 4)$ \\
\hline
\end{tabular}

Table 6

These algebras must act effectively on the internal spaces of $A d S_{n}$ backgrounds.

\begin{tabular}{|c|c|c|c|c|}
\hline \multicolumn{5}{|c|}{ Isometry algebras of internal spaces } \\
\hline$N$ & $\mathrm{AdS}_{4}$ & $\mathrm{AdS}_{5}$ & $\mathrm{AdS}_{6}$ & $\mathrm{AdS}_{7}$ \\
\hline 4 & $\{0\}$ & - & - & - \\
\hline 8 & $\mathfrak{s o}(2)$ & $\mathfrak{u}(1)$ & - & - \\
\hline 12 & $\mathfrak{s o}(3)$ & - & - & - \\
\hline 16 & $\mathfrak{s o}(4)$ & $\mathfrak{u}(2)$ & $\mathfrak{s o}(3)$ & $\mathfrak{s o}(3)$ \\
\hline 20 & $\mathfrak{s o}(5)$ & - & - & - \\
\hline 24 & $\mathfrak{s o}(6)$ & $\mathfrak{u}(3)$ & - & - \\
\hline 28 & $\mathfrak{s o}(7)$ & - & - & - \\
\hline 32 & $\mathfrak{s o}(8)$ & $\mathfrak{s u}(4)$ & - & $\mathfrak{s o}(5)$ \\
\hline
\end{tabular}

Table 7

$\left(\mathfrak{g}_{L} / \mathfrak{c}_{L}\right)_{0}=\mathfrak{s o}(1,2) \oplus \mathfrak{t}_{0} / \mathfrak{c}_{L}$, where there may be a central term $\mathfrak{c}_{L}$. The superalgebras osp* $(4 \mid 2 k)$ are different real forms of the osp $(4 \mid 2 k)$ superalgebras, see Table 5.

\begin{tabular}{|c|c|}
\hline \multicolumn{2}{|c|}{ Killing superalgebras of $d=10,11$ warped $\mathrm{AdS}_{2}$ and $A d S_{3}$ solutions } \\
\hline$N_{L}$ & $\mathfrak{g}_{L} / \mathfrak{c}_{L}$ \\
\hline $2 k$ & $\mathfrak{o s p}(k \mid 2)$ \\
\hline $4 k, k>1$ & $\mathfrak{s l}(k \mid 2)$ \\
\hline $8 k, k>1$ & $\mathfrak{o s p}^{*}(4 \mid 2 k)$ \\
\hline 16 & $f(4)$ \\
\hline 14 & $\mathfrak{g}(3)$ \\
\hline 8 & $\mathfrak{D}(2,1, \alpha)$ \\
\hline 8 & $\mathfrak{s l}(2 \mid 2) / 1_{4 \times 4}$ \\
\hline
\end{tabular}

of the super-Jacobi identities (300) of the Killing superalgebra. The remaining commutator $\left[\mathfrak{t}_{0}, \mathfrak{t}_{0}\right]$ also follows from the super-Jacobi identities. The details of this computation can be found in [255]. It is remarkable that for each $\operatorname{AdS}_{n}$ background the Killing superalgebra is specified uniquely by the number $N$ of supersymmetries that are preserved. Another important consequence of the computation of the super-Jacobi identities is that the Lie algebra $t_{0}$ acts (almost) effectively on the internal space, i.e. all elements of $\mathrm{t}_{0}$ generate a non-trivial vector field on $N^{d-n}$. If this is not the case, the super-Jacobi identities cannot be satisfied.

\subsubsection{Killing superalgebras of $\mathrm{AdS}_{2}$ and $A d S_{3}$ backgrounds}

Let us now turn to investigate the Killing superalgebras of warped $A d S_{3}$ backgrounds. $A d S_{3}$ is locally a group manifold and the Killing superalgebra $\mathfrak{g}$ decomposes as $\mathfrak{g}=\mathfrak{g}_{L} \oplus \mathfrak{g}_{R}$ into left and right superalgebras, $\left[\mathfrak{g}_{L}, \mathfrak{g}_{R}\right]=0$. The left superalgebra $\mathfrak{g}_{L}$ is associated with the $\sigma_{+}$Killing spinors while $\mathfrak{g}_{L}$ is associated with the $\tau_{+}$Killing spinors. It suffices to identify only $\mathfrak{g}_{L}$ as the same techniques can be used to identify the $\mathfrak{g}_{R}$ Killing superalgebras. The list of $\mathfrak{g}_{L}$ Killing superalgebras is the same as that of the $\mathfrak{g}_{R}$ Killing superalgebras. Though a given background may exhibit a different $\mathfrak{g}_{L}$ from a $\mathfrak{g}_{R}$ Killing superalgebra. Assuming the conditions (403) on the bilinears, the $\mathfrak{g}_{L}$ Killing superalgebras of $A d S_{3}$ backgrounds are given in Table 7. Furthermore, the isometry algebras of the internal space are given in Table 8. The Killing superalgebras of $A_{d S_{2}}$ backgrounds with compact, without boundary, internal space can be identified with the left copies $\mathfrak{g}_{L}$ of the Killing superalgebras of warped $\mathrm{AdS}_{3}$ backgrounds.

The identification of Killing superalgebras in Table 7 for warped $A d S_{2}$ and $A d S_{3}$ backgrounds is more involved than that presented in the previous section for warped $A d S_{n}, n>3$ backgrounds. To outline the main steps of the proof, consider first the Killing superalgebra $\mathfrak{g}_{L}$ of $N=2$ warped $A_{d S} S_{3}$ backgrounds. A direct computation reveals that

$$
\left\{Q_{A}, Q_{B}\right\}=V_{A B}, \quad\left[V_{A B}, Q_{C}\right]=-\ell^{-1}\left(\epsilon_{C A} Q_{B}+\epsilon_{C B} Q_{A}\right),
$$


Table 8

$\mathfrak{s p}^{*}(k)$ is the compact symplectic algebra with (real) dimension $k(2 k+1)$ which is a real form of $\mathfrak{s p}(2 k)$.

\begin{tabular}{|c|c|c|c|}
\hline \multicolumn{4}{|c|}{ Isometries of internal spaces } \\
\hline$N_{L}$ & $\mathfrak{g}_{L} / \mathfrak{c}_{L}$ & $\left(\mathfrak{t}_{L}\right)_{0} / \mathfrak{c}_{L}$ & $\operatorname{dim} \mathfrak{c}_{L}$ \\
\hline $2 k$ & $\mathfrak{o s p}(k \mid 2)$ & $\mathfrak{s o}(k)$ & 0 \\
\hline $4 k, k>2$ & $\mathfrak{s l}(k \mid 2)$ & $\mathfrak{u}(k)$ & 0 \\
\hline $8 k, k>1$ & $\mathfrak{o s p}(4 \mid 2 k)$ & $\mathfrak{s p}^{*}(k) \oplus \mathfrak{s p}^{*}(1)$ & 0 \\
\hline 16 & $\mathfrak{f}(4)$ & $\mathfrak{s p i n}(7)$ & 0 \\
\hline 14 & $\mathfrak{g}(3)$ & $\mathfrak{g}_{2}$ & 0 \\
\hline 8 & $\mathfrak{D}(2,1, \alpha)$ & $\mathfrak{s o}(3) \oplus \mathfrak{s o}(3)$ & 0 \\
\hline 8 & $\mathfrak{s l}(2 \mid 2) / 1_{4 \times 4}$ & $\mathfrak{s u}(2)$ & $\leq 3$ \\
\hline
\end{tabular}

where $A, B, C=1,2, V_{A B}$ are the generators of $\mathfrak{g}_{0}=\mathfrak{s o}(1,2)=\mathfrak{s p}(2)$ and $Q_{A}$ are odd generators of the superalgebra associated to the two Killing spinors (387) constructed from $\sigma_{ \pm}$. There are no internal space isometries generated from vector bilinears and so $\mathfrak{t}_{0}=\{0\}$. This superalgebra is isomorphic to osp $(1 \mid 2)$. Note that in this section $A, B, C$ are not frame coframe indices.

Suppose now that we have $N=2 k$ supersymmetries. As the dependence of the Killing spinors (387) on the $A_{d S_{3}}$ coordinates is known, one finds after a direct computation that

$$
\begin{aligned}
& \left\{Q_{A r}, Q_{B s}\right\}=V_{A B} \delta_{r s}+\epsilon_{A B} \tilde{V}_{r s}, \\
& {\left[V_{A B}, Q_{C r}\right]=-\ell^{-1}\left(\epsilon_{C A} Q_{B r}+\epsilon_{C B} Q_{A r}\right),}
\end{aligned}
$$

where $r, s=1, \ldots, k$ and $\tilde{V}_{r s} \in \mathfrak{t}_{0}$. It remains to compute $\left[\tilde{V}_{r s}, Q_{A t}\right]$. As the spinorial Lie derivative along isometries $\tilde{X}$ of the internal space does not change the functional dependence of the Killing spinors $\epsilon$ on the $A_{d S} S_{3}$ coordinates, one concludes that

$$
\left[\tilde{V}_{r s}, Q_{A t}\right]=-\ell^{-1}\left(\delta_{t r} Q_{A s}-\delta_{t s} Q_{A r}\right)+\ell^{-1} \alpha_{r s t}{ }^{p} Q_{A p},
$$

for some structure constants $\alpha$ which remain to be determined. The super-Jacobi identities (300) together with the identity

$$
\left\langle\mathcal{L}_{\tilde{X}} \sigma_{+}^{r}, \sigma_{+}^{s}\right\rangle+\left\langle\sigma_{+}^{r}, \mathcal{L}_{\tilde{X}} \sigma_{+}^{s}\right\rangle=0,
$$

imply that $\alpha$ is a 4 -form. To prove (411), take the Lie derivative of the normalization condition of the $\sigma_{+}$Killing spinors in (406) with respect to internal space isometries $\tilde{X}$.

Furthermore $\alpha$ is invariant under the representation $D$ of $\mathfrak{t}_{0}$ on $\mathfrak{g}_{1}$, where

$$
D\left(\tilde{V}_{r s}\right) Q_{A t}:=\left[\tilde{V}_{r s}, Q_{A t}\right] .
$$

Note that $\mathfrak{g}$ may have a centre $\mathfrak{c}_{L}:=\left\{\tilde{V} \in \mathfrak{t}_{0} \mid D(\tilde{V})=0\right\}$. It turns out that $\mathfrak{c}_{L}=\{0\}$ apart from one case where it can have a dimension of at most 3 , see Table 8 .

The key observation which identifies the representations $D$ that can occur is that $D$ acts transitively (and effectively) on spheres in $\mathbb{R}^{\frac{N}{2}}$. For this it suffices to show that given two linearly independent vectors $u, w \in \mathbb{R}^{\frac{N}{2}}$, there is an element $R(u, w) \in \mathfrak{t}_{0}$ such that $R(u, w)$ generates $S O(2)$ rotations on the 2-plane spanned by $u$ and $w$ in $\mathbb{R}^{\frac{N}{2}}$. The statement then follows as such $S O(2)$ rotations act transitively on all directions in the 2-plane spanned by $u$ and $w$.

For this set $R(u, w)=u^{r} w^{s} \tilde{V}_{r s}$ and $p \cdot Q_{A}=p^{r} Q_{A r}$, and observe that

$$
\begin{aligned}
D(R(u, w))\left(p \cdot Q_{A}\right) & =\left[u^{r} w^{s} \tilde{V}_{r s}, p^{t} Q_{A t}\right] \\
& =-\ell^{-1}\left(p \cdot u w^{r}-p \cdot w u^{r}\right) Q_{A r},
\end{aligned}
$$

for any $p$ that lies in the 2-plane spanned by $u$ and $w$ as $\alpha(u, w, p, \cdot)=0$. So indeed $R(u, w)$ acts as an infinitesimal orthogonal rotation on the 2-plane spanned by $u$ and $w$. As this can be done for any $u, w \in \mathbb{R}^{\frac{N}{2}}$, it follows that $\mathfrak{t}_{0}$ acts transitively on $S^{\frac{N}{2}-1} \subset \mathbb{R}^{\frac{N}{2}}$. The groups that act effectively and transitively on spheres have been classified in [257] and they have been used in the Berger classification of irreducible simply connected Riemannian manifolds [258]. They are given in Table 9. Some further analysis which can be found in [255] reveals that only some of these groups occur in the investigation of superalgebras and the final result is described in Table 7.

\section{7. $N>16$ AdS backgrounds}

As an application of the technology developed so far, we shall provide a classification of smooth warped AdS backgrounds with compact, without boundary, internal space that preserve $N>16$ supersymmetries in $d=11$ and $d=10$ type II supergravities [10,259-261]. In particular, one can show the following.

- There are no warped $A d S_{n}, n=2,3,6$, backgrounds that preserve $N>16$ supersymmetries. 
Table 9

spin(9) cannot be realized as a symmetry of the internal space of warped $A d S_{2}$ and $A d S_{3}$ backgrounds as there are no such maximally supersymmetric backgrounds.

\begin{tabular}{lll}
\hline \multicolumn{2}{l}{ Lie algebras of groups acting transitively on spheres } & \\
\hline Algebra & Sphere & $N / 2$ \\
\hline $\mathfrak{s o}(k)$ & $S^{k-1}$ & $k$ \\
$\mathfrak{u}(k)$ & $S^{2 k-1}$ & $2 k$ \\
$\mathfrak{s u}^{*}(k)$ & $S^{2 k-1}$ & $2 k$ \\
$\mathfrak{s p}^{*}(k) \oplus \mathfrak{s p}^{*}(1)$ & $S^{4 k-1}$ & $4 k$ \\
$\mathfrak{s p}^{*}(k) \oplus \mathfrak{u}(1)$ & $S^{4 k-1}$ & $4 k$ \\
$\mathfrak{s p}^{*}(k)$ & $S^{4 k-1}$ & $4 k$ \\
$\mathfrak{g}_{2}$ & $S^{6}$ & 7 \\
$\mathfrak{s p i n}(7)$ & $S^{7}$ & 8 \\
$\mathfrak{s p i n}(9)$ & $S^{15}$ & 16 \\
\hline
\end{tabular}

- The only warped $A_{d S_{4}}$ backgrounds that preserve $N>16$ supersymmetries are locally isometric to the $N=24$, $A d S_{4} \times \mathbb{C} P^{3}$, solution of IIA supergravity of [262] and the maximally supersymmetric solution, $A d S_{4} \times S^{7}$, of $d=11$ supergravity.

- The only warped $A d S_{7}$ backgrounds that preserve $N>16$ supersymmetries are locally isometric to the maximally supersymmetric solution, $A d S_{7} \times S^{4}$, of $d=11$ supergravity.

- The only warped $A d S_{5}$ backgrounds that preserve $N>16$ supersymmetries are locally isometric to the maximally supersymmetric solution, $A d S_{5} \times S^{5}$, of IIB supergravity.

The result above follows immediately for warped $A d S_{6}$ and $A d S_{7}$ backgrounds as these preserve either 16 or 32 supersymmetries. Therefore if they exist, they must be maximally supersymmetric. The maximally supersymmetric backgrounds have been classified in [10] and this has already been reviewed in Section 9 yielding the result stated above.

The next new case that arises is that of warped $A d S_{5}$ backgrounds that may preserve $N=24$ supersymmetries. The maximally supersymmetric $A d S_{5}$ backgrounds have already been dealt with in Section 9 as part of the classification of maximally supersymmetric backgrounds in $d=10$ and $d=11$ supergravities.

\subsubsection{A non-existence theorem for warped $N=24 \mathrm{AdS}_{5}$ backgrounds}

Here we shall present the main points of the proof in the context of $d=11$ supergravity, see also [259] and for the rest of the theories. The fields of warped $A d S_{5}$ backgrounds in $d=11$ supergravity are

$$
\begin{aligned}
& d s^{2}=2 d u(d r+r h)+A^{2}\left(d s^{2}+e^{\frac{2 z}{\ell}}\left(d x^{a}\right)^{2}\right)+d s^{2}\left(M^{6}\right), \\
& F=Z, \quad h=-\frac{2}{\ell} d z-2 A^{-1} d A,
\end{aligned}
$$

and the supercovariant connection on the internal space is

$$
D_{i}^{( \pm)}=D_{i} \pm \frac{1}{2} \partial_{i} \log A-\frac{1}{288} \not / Z_{i}+\frac{1}{36} Z_{i} .
$$

Using that $\left\|\sigma_{+}\right\|^{2}$ is constant, see (405), and $D_{i}^{(+)} \sigma_{+}=0$, one finds that

$$
-\left\|\sigma_{+}\right\|^{2} \partial_{i} \log A+\frac{1}{144}\left\langle\sigma_{+}, \not / Z_{i} \sigma_{+}\right\rangle=0 .
$$

The Killing vectors along the internal space are

$$
\tilde{X}_{i}=A\left\langle\sigma_{+}, \Gamma_{z 12 i} \sigma_{+}\right\rangle \text {. }
$$

Using this, (416) can be written as

$$
i_{\tilde{X}} \star_{6} Z=6\left\|\sigma_{+}\right\|^{2} d A .
$$

Taking the inner derivation of the above equation with $\tilde{X}$, one also finds that

$$
i_{\tilde{X}} d A=0 .
$$

An adaptation of the homogeneity theorem argument [67], reviewed in Section 10.4, leads to the conclusion that $\tilde{X}$ span the tangent space of the internal space. As a result $Z=0$ and $A$ is constant. However in such a case, the warp factor field equation

$$
D^{2} \log A=-\frac{4}{\ell^{2} A^{2}}-5(d \log A)^{2}+\frac{1}{144} Z^{2},
$$

cannot be satisfied. This excludes the existence of warped $A d S_{5}$ backgrounds in $d=11$ supergravity that preserve $N>16$ supersymmetries. 
Table 10

7-dimensional compact, simply connected, homogeneous spaces.

\begin{tabular}{ll}
\hline & $M^{7}=G / H$ \\
\hline$(1)$ & $\frac{S p i n(8)}{S p i n(7)}=S^{7}$, symmetric space \\
$(2)$ & $\frac{S p i n(7)}{G_{2}}=S^{7}$ \\
$(3)$ & $\frac{S U(4)}{S U(3)}$ diffeomorphic to $S^{7}$ \\
$(4)$ & $\frac{S p(2)}{S p(1)}$ diffeomorphic to $S^{7}$ \\
$(5)$ & $\frac{S p(2)}{S p(1) \max }$, Berger space \\
$(6)$ & $\frac{S p(2)}{\Delta(S p(1))}=V_{2}\left(\mathbb{R}^{5}\right)$, not spin \\
$(7)$ & $\frac{S U(3)}{\Delta_{k, l}(U(1))}=W^{k, l} \quad k, l$ coprime, Aloff-Wallach space \\
$(8)$ & $\frac{S U(2) \times S U(3)}{\Delta_{k, l}(U(1)) \cdot(1 \times S U(2))}=N^{k, l} k, l$ coprime \\
$(9)$ & $\frac{S U(2)^{3}}{\Delta_{p, q, r}\left(U(1)^{2}\right)}=Q^{p, q, r} p, q, r$ coprime \\
$(10)$ & $M^{4} \times M^{3}, M^{4}=\frac{S p i n(5)}{S p i n(4)}, \frac{S U(3)}{S(U(1) \times U(2))}, \frac{S U(2)}{U(1)} \times \frac{S U(2)}{U(1)}$ \\
& $M^{3}=S U(2), \frac{S U(2) \times S U(2)}{\Delta(S U(2))}$ \\
$(11)$ & $M^{5} \times \frac{S U(2)}{U(1)}, M^{5}=\frac{S p i n(6)}{S p i n(5)}, \frac{S U(3)}{S U(2)}, \frac{S U(2) \times S U(2)}{\Delta k, l(U(1))}, \frac{S U(3)}{S O(3)}$ \\
\hline
\end{tabular}

\subsubsection{Existence and uniqueness theorems for warped $N>16 \mathrm{AdS}_{4}$ backgrounds}

The proof presented in the previous section to find the $N>16$ warped $A d S_{5}$ backgrounds cannot be adapted to investigate the warped $A_{d} S_{4}$ backgrounds that preserve $N>16$ supersymmetries. Instead, a more detailed investigation is required of the homogeneous structure of spacetime which is implied by the homogeneity theorem.

First, one establishes that the warp factor $A$ is constant. To prove this one first uses the algebraic KSE, $\Xi^{(+)} \sigma_{+}=0$, to find $i_{\tilde{X}} d A=0$, where $\tilde{X}$ is a Killing vector field of the internal space. As $N>16$, the homogeneity theorem implies that $\tilde{X}$ span the tangent of the internal space which gives that $A$ is constant. Thus the spacetime is a product $A d S_{4} \times N^{d-4}$ and $N^{d-4}$ is a homogeneous space $G / H$.

Therefore, one has to identify the homogeneous spaces $G / H$ that can occur as internal spaces of $N>16 \mathrm{AdS}_{4}$ backgrounds. To do this, one uses the classification of all Killing superalgebras of AdS backgrounds and in particular that of the Lie algebra of isometries of the internal spaces tabulated in Table 6 . This together with the homogeneity theorem implies that $\mathfrak{L i e} G=\mathfrak{t}_{0}=\mathfrak{s o}(N / 4)$ for $A d S_{4}$ backgrounds with $N>16$.

Further progress is made by utilizing the classification of homogeneous spaces, see [233,263,264]. In particular for $d=11$ supergravity $\mathrm{AdS}_{4}$ backgrounds have a 7-dimensional homogeneous internal space. All simply connected 7dimensional homogeneous spaces have been tabulated in Table 10. It is straightforward to observe that the requirement that $\mathfrak{L i e} G=\mathfrak{s o}(N / 4)$ restricts the number of homogeneous spaces that can occur as internal spaces of $A_{d} S_{4}$ backgrounds that preserve $N>16$ supersymmetries to the first five in Table 10. The rest of the proof proceeds with the analysis of each case separately and gives that the only warped $A d S_{4}$ backgrounds of $d=11$ supergravity are locally isometric to the maximally supersymmetric $\mathrm{AdS}_{4} \times S^{7}$ solution.

One uses a similar methodology to establish the classification statement for $A_{d S_{4}}$ backgrounds in Section 12.7 for (massive) IIA and IIB supergravities. This method can also be extended to prove a non-existence result for $\operatorname{AdS}_{2}$ and $\mathrm{AdS}_{3}$ backgrounds that preserve $N>16$ supersymmetries [261,265].

\section{Conclusions}

Significant progress has been made in the last 15 years to classify the supersymmetric backgrounds in all supergravity theories and explore their applications in the context of string theory, M-theory, gauge theory, black holes and the AdS/CFT correspondence. The task has been completed for a substantial class of theories which include all those with a small number of supercharges in each spacetime dimension. This has given an insight into the structure of all supersymmetric solutions and has led to a plethora of existence and uniqueness theorems for backgrounds, including those of black holes and warped AdS spaces, which otherwise would have been out of reach. We presented the classification of supersymmetric solutions in terms of only a few examples and there are many other significant theories that could have been included. However, we endeavoured to be concise and give a taste of how such proofs and calculations can be carried out.

The emphasis in this review has been to describe the bilinears and spinorial geometry methods that have been used to solve the KSEs of supergravity theories. These methods cover all the theories that have been treated in the literature. The solution of the KSEs is the first key step towards the classification of supersymmetric backgrounds. After obtaining the solution of the KSEs, we proceeded to explain how to use it to identify the geometry of the spacetime.

For the description of the geometry of supersymmetric backgrounds, we have used partly local techniques and partly borrowed the language of bundles and G-structures. These are sufficient for all practical purposes. We also presented a taste 
of how powerful global techniques like index theory, Lichnerowicz type theorems and the Hopf maximum principle can be used to prove general properties of supersymmetric backgrounds.

We have included in the review some other key properties of the supersymmetric solutions like their Killing superalgebras and the homogeneity theorem, and also the non-existence theorem for de-Sitter and Minkowski flux compactifications in supergravity. As applications, we demonstrated that the emergence of conformal symmetry near supersymmetric Killing horizons is a generic phenomenon in supergravity, which does not depend on the details of the black hole solutions. We also classified the warped AdS backgrounds that preserve $N>16$ supersymmetries in $d=11$ and $d=10$ supergravities.

One of the last remaining challenges in this field is to solve the KSEs in $d=11$ and $d=10$ type II supergravities for backgrounds preserving any number of supersymmetries. As has already been described, the geometry of $N=1$ backgrounds is known and there is a classification of the maximally and nearly maximally supersymmetric backgrounds. However very little is known about the geometry of solutions that preserve an intermediate number of supersymmetries. The final objective is to give a description of the geometries similar to those of heterotic backgrounds as presented in Section 7. It is encouraging that there are strong constraints on the existence of special backgrounds that preserve $N>16$ supersymmetries like those for the AdS backgrounds we have described. This indicates that the bulk of the task will be to understand the geometry of backgrounds that preserve $N \leq 16$ supersymmetries.

Of course in many applications the interest is focused on special types of solutions, e.g. black holes or warped Minkowski and AdS flux compactifications. For those there are many simplifications and a complete identification of all such backgrounds may be possible. It is encouraging that there is increasing detail in the understanding of AdS backgrounds which have applications in the context of the AdS/CFT correspondence. It is very likely that in the next few years there will be a complete understanding of the structure of all such solutions.

\section{Acknowledgements}

UG is supported by the Swedish Research Council. GP would like to thank the Yau Mathematical Sciences Center, Tsinghua University and Theoretical Physics Department at CERN for hospitality and support. Part of this review has been presented by one of us, GP, at the "School on Theoretical Approaches to Black Hole Physics" Mesoamerican Centre for Theoretical Physics, Universidad Autonoma de Chiapas, Sept 2017, and as a short course at Tsinghua University. JG is supported by the STFC (UK) Consolidated Grant ST/L000490/1. GP is partially supported from the STFC (UK) consolidated grant ST/P000258/1.

\section{Declarations of interest}

None.

\section{Appendix A. Notation for forms}

Let $M$ be a manifold with a (local) coframe $\mathbf{e}^{i}$ and coordinates $y^{l}$. The exterior derivative on a k-form,

$$
\omega=\frac{1}{k !} \omega_{I_{1} \ldots I_{k}} d y^{I_{1}} \wedge \cdots \wedge d y^{I_{k}}=\frac{1}{k !} \omega_{i_{1} \ldots i_{k}} \mathbf{e}^{i_{1}} \wedge \cdots \wedge \mathbf{e}^{i_{k}},
$$

is

$$
d \omega:=\frac{1}{k !} \partial_{I_{1}} \omega_{I_{2} \ldots I_{k+1}} d y^{I_{1}} \wedge \cdots \wedge d y^{I_{k+1}} .
$$

Therefore, one has $(d \omega)_{I_{1} \ldots I_{k+1}}=(k+1) \partial_{\left[I_{1}\right.} \omega_{\left.I_{2} \ldots I_{k+1}\right]}$. The inner derivation $i_{X}$ of a $\mathrm{k}$-form $\omega$ with respect to a vector field $X$ is

$$
i_{X} \omega:=\frac{1}{(k-1) !} X^{j} \omega_{j i_{1} \ldots i_{k-1}} \mathbf{e}^{i_{1}} \wedge \cdots \wedge \mathbf{e}^{i_{k-1}} .
$$

Furthermore, it is convenient to set

$$
\omega^{2}:=\omega_{i_{1} \ldots i_{k}} \omega^{i_{1} \ldots i_{k}}, \quad \omega_{i_{1} i_{2}}^{2}:=\omega_{i_{1} j_{1} \ldots j_{k-1}} \omega_{i_{2}}^{j_{1} \ldots j_{k-1}},
$$

where the indices are raised with respect to a metric, $d s^{2}=g_{i j} \mathbf{e}^{i} \mathbf{e}^{j}$, on $M$. The inner product of two k-forms $\chi$ and $\omega$ is

$$
(\chi, \omega):=\frac{1}{k !} \chi_{i_{1} \ldots i_{k}} \omega^{i_{1} \ldots i_{k}} .
$$

Given a volume form $d v o l=\frac{1}{n !} \epsilon_{i_{1} \ldots i_{n}} d x^{i_{1}} \wedge \cdots \wedge d x^{i_{n}}$, the Hodge dual of the k-form $\omega$ is defined as

$$
\chi \wedge * \omega=(\chi, \omega) d v o l,
$$

for every k-form $\chi$.

It is well-known that for every form $\omega$, one can define a Clifford algebra element $\phi$ given by

$$
\phi:=\omega_{i_{1} \ldots i_{k}} \Gamma^{i_{1} \ldots i_{k}},
$$


where $\Gamma^{i}, i=1, \ldots n$, are the Dirac gamma matrices. In addition we have introduced the notation

$$
\phi_{i_{1}}:=\omega_{i_{1} i_{2} \ldots i_{k}} \Gamma^{i_{2} \ldots i_{k}}, \quad I \omega_{i_{1}}:=\Gamma_{i_{1}}{ }^{i_{2} \ldots i_{k+1}} \omega_{i_{2} \ldots i_{k+1}} .
$$

This has significantly shortened some of expressions for the KSEs.

\section{Appendix B. Spinors and forms}

There is an extensive literature on the representations of Clifford algebras and Spin groups, see e.g. [266,267]. Here the emphasis is on an explicit realization of the spinor representations of the Spin groups in terms of forms which is used in the spinorial geometry approach to solving KSEs. We follow the construction of [268] and [9] for the Euclidean and Lorentzian cases, respectively.

\section{B.1. Euclidean}

To realize the Dirac representation, ${ }^{c} \Delta$, of $\operatorname{Spin}(2 n)$ in terms of forms, consider the space of all (complex) forms on $\mathbb{C}^{n}$, $\Lambda^{*}\left(\mathbb{C}^{n}\right)$, equipped with a Hermitian inner product $\langle\cdot, \cdot\rangle$ and $\operatorname{set}^{c} \Delta=\Lambda^{*}\left(\mathbb{C}^{n}\right)$. Then gamma matrices act as

$$
\Gamma_{i} \zeta=e_{i} \wedge \zeta+i_{e_{i}} \zeta, \quad \Gamma_{i+n} \zeta=i\left(e_{i} \wedge \zeta-i_{e_{i}} \zeta\right), \quad i=1, \ldots, n,
$$

where $e_{i}$ is a Hermitian basis in $\Lambda^{1}\left(\mathbb{C}^{n}\right),\left\langle e_{i}, e_{j}\right\rangle=\delta_{i j}$, and $\zeta$ is a multi-degree form in $\Lambda^{*}\left(\mathbb{C}^{n}\right)$. The operation $i_{e_{i}}$ is the inner derivation with respect to the vector constructed from $e_{i}$ using $\langle\cdot, \cdot\rangle$. The gamma matrices, $\Gamma_{A}, A=1, \ldots, 2 n$, defined above are Hermitian with respect to $\langle\cdot, \cdot\rangle$ and satisfy the Clifford algebra relations $\Gamma_{A} \Gamma_{B}+\Gamma_{B} \Gamma_{A}=2 \mathbf{1} \delta_{A B}$. It is usual to label bases in the space of forms with upper indices. Here the basis $\left\{e_{i}\right\}$ has been labelled with lower indices in order to distinguish the notation of spinors in terms of forms from that used for forms on the spacetime.

In the Euclidean case, the Dirac inner product, $D$, is identified with the Hermitian inner product $\langle\cdot, \cdot\rangle$ on $\Lambda^{*}\left(\mathbb{C}^{n}\right), D(\cdot, \cdot)=$ $\langle\cdot, \cdot\rangle$. As the gamma matrices are Hermitian with respect to $\langle\cdot, \cdot\rangle$,

$$
\left\langle\Gamma_{A B} \eta, \zeta\right\rangle+\left\langle\eta, \Gamma_{A B} \zeta\right\rangle=0,
$$

and so $D$ is invariant under the Lie algebra $\mathfrak{s p i n}(2 n)$ of $\operatorname{Spin}(2 n)$. In fact $D$ is invariant under the action of $\operatorname{Spin}(2 n)$ which is the double cover of $S O(2 n)$.

The Dirac representation of $\operatorname{Spin}(2 n)$ is reducible and decomposes as ${ }^{c} \Delta={ }^{c} \Delta^{+} \oplus^{c} \Delta^{-}$into chiral and anti-chiral Weyl representations according to the decomposition of $\Lambda^{*}\left(\mathbb{C}^{n}\right)$ into forms of even and odd degree, ${ }^{c} \Delta^{+}=\Lambda^{\mathrm{ev}}\left(\mathbb{C}^{n}\right)$ and ${ }^{c} \Delta^{-}=\Lambda^{\text {odd }}\left(\mathbb{C}^{n}\right)$, respectively.

Next consider the linear maps $a:=\prod_{i=1}^{n} \Gamma_{i}$ and $b:=\prod_{i=1}^{n} \Gamma_{i+n}$. There are two $\operatorname{Spin}(2 n)$-invariant bi-linears, the Majorana inner products, which can be constructed on ${ }^{c} \Delta$ as

$$
\mathrm{A}(\eta, \zeta):=\left\langle a \eta^{*}, \zeta\right\rangle, \quad \mathrm{B}(\eta, \zeta):=\left\langle b \eta^{*}, \zeta\right\rangle,
$$

where $\eta, \zeta \in{ }^{c} \Delta$ and $\eta^{*}$ is the complex conjugate of $\eta$. The bi-linearity of $A$ and $B$ is assured because $\langle\cdot, \cdot\rangle$ is anti-linear in the left entry. A straightforward computation following the definitions reveals that

$$
\begin{aligned}
& \mathrm{A}\left(\eta, \Gamma_{\mathrm{A}} \zeta\right)=(-1)^{n-1} \mathrm{~A}\left(\Gamma_{A} \eta, \zeta\right), \mathrm{A}\left(\eta, \Gamma_{A} \zeta\right)=(-1)^{\frac{(n+2)(n-1)}{2}} \mathrm{~A}\left(\zeta, \Gamma_{A} \eta\right), \\
& \mathrm{B}\left(\eta, \Gamma_{A} \zeta\right)=(-1)^{n} \mathrm{~B}\left(\Gamma_{A} \eta, \zeta\right), \mathrm{B}\left(\eta, \Gamma_{A} \zeta\right)=(-1)^{\frac{n(n-1)}{2}} \mathrm{~B}\left(\zeta, \Gamma_{A} \eta\right) .
\end{aligned}
$$

This confirms that both A and B are invariant under $\operatorname{Spin}(2 n)$ and in addition that one of them is also invariant under $\operatorname{Pin}(2 n)$. Note that the Lie algebra of the Pin group is spanned by $\Gamma_{A B}$ and $\Gamma_{A}$. Furthermore, after lowering the spinor indices of the gamma matrices with respect to $\mathrm{A}$ or $\mathrm{B}$, these become either symmetric or skew-symmetric.

So far, we have dealt with complex representations of Spin groups. Real representations exist whenever a reality condition can be imposed on the complex representations, i.e. there is an antilinear map $\mathcal{R}$ which commutes with the action of Spin on ${ }^{c} \Delta$ and $\mathcal{R}^{2}=\mathbf{1}$. Such maps are not unique since if $\mathcal{R}$ is a reality condition, then $e^{i \theta} \mathcal{R}$ is also a reality condition for any angle $\theta$. To proceed consider the anti-linear maps $r_{\mathrm{A}}=a *$ and $r_{\mathrm{B}}=b *$. One can verify that

$$
\begin{array}{ll}
r_{\mathrm{A}}^{2}=(-1)^{\frac{n(n-1)}{2}} \mathbf{1}, & r_{\mathrm{A}} \Gamma_{\mathrm{A}}=(-1)^{n-1} \Gamma_{\mathrm{A}} r_{\mathrm{A}}, \\
r_{\mathrm{B}}^{2}=(-1)^{\frac{n(n+1)}{2}} \mathbf{1}, & r_{\mathrm{B}} \Gamma_{\mathrm{A}}=(-1)^{n} \Gamma_{\mathrm{A}} r_{\mathrm{B}} .
\end{array}
$$

Therefore there are real representations with reality conditions either $\mathcal{R}_{\mathrm{A}}=e^{i \theta} r_{\mathrm{A}}$ or $\mathcal{R}_{\mathrm{B}}=e^{i \theta} r_{\mathrm{B}}$ provided that $\left[\frac{n}{2}\right] \in 2 \mathbb{Z}$. If in addition $\mathcal{R}^{c} \Delta^{ \pm} \subset{ }^{c} \Delta^{ \pm}$, then there are Majorana-Weyl representations. So there are Majorana-Weyl representations iff $n \in 4 \mathbb{Z}$.

There is an oscillator basis in the space of Dirac spinors which we use to solve the KSEs. To see this, write the gamma matrices (B.1) in a Hermitian basis as

$$
\gamma_{\alpha}=\frac{1}{\sqrt{2}}\left(\Gamma_{\alpha}-i \Gamma_{\alpha+n}\right)=\sqrt{2} e_{\alpha} \wedge, \quad \gamma_{\bar{\alpha}}=\frac{1}{\sqrt{2}}\left(\Gamma_{\alpha}+i \Gamma_{\alpha+n}\right)=\sqrt{2} i_{e_{\alpha}},
$$


and set $\gamma^{\bar{\alpha}}=\delta^{\bar{\alpha} \beta} \gamma_{\beta}$ and $\gamma^{\alpha}=\delta^{\alpha \bar{\beta}} \gamma_{\bar{\beta}}$. One can verify that $\gamma_{\alpha} \gamma_{\beta}+\gamma_{\beta} \gamma_{\alpha}=0$ and $\gamma_{\alpha} \gamma_{\bar{\beta}}+\gamma_{\bar{\beta}} \gamma_{\alpha}=2 \mathbf{1} \delta_{\alpha \bar{\beta}}$. It is clear that the whole Dirac representation can be constructed by acting with the "creation operators" $\gamma^{\bar{\alpha}}$ on the Clifford vacuum represented by the 0 -degree form 1 . In curved spaces, a choice of a spacetime coframe which is compatible with the realization of gamma matrices as in (B.6) is referred to as either an (almost) Hermitian coframe or a "spinorial geometry coframe".

One way to realize the spinor representation of $\operatorname{Spin}(2 n+1)$ in terms of forms is to add an additional gamma matrix $\Gamma_{2 n+1}$ to those of $\operatorname{Spin}(2 n)$ proportional to $\prod_{A=1}^{2 n} \Gamma_{A}$. The Dirac representation of $\operatorname{Spin}(2 n+1)$ will coincide with the complex representation of $\operatorname{Pin}(2 n)$. Moreover for $n$ even, $\operatorname{Sin}(2 n+1)$ will admit a Majorana representation provided that $\operatorname{Pin}(2 n)$ admits one. Similarly for $n$ odd, $\operatorname{Spin}(2 n+1)$ will admit a Majorana representation provided that $\operatorname{Pin}(2 n)$ admits one.

\section{B.2. Lorentzian}

The realization of spinor representations of $\operatorname{Spin}(2 n-1,1)$ in terms of forms proceeds as in the Euclidean case described in the appendix above. Let $\mathbb{C}^{n}$ be equipped with a Hermitian inner product $\langle\cdot, \cdot\rangle$ and a Hermitian basis $\left\{e_{1}, \ldots, e_{n}\right\}$. The Dirac representation is identified with ${ }^{c} \Delta=\Lambda^{*}\left(\mathbb{C}^{n}\right)$ and the gamma matrices act as

$$
\begin{aligned}
\Gamma_{0} \zeta & =-e_{n} \wedge \zeta+i_{e_{n}} \zeta, \quad \Gamma_{n} \zeta=e_{n} \wedge \zeta+i_{e_{n}} \zeta, \\
\Gamma_{i} \zeta & =e_{i} \wedge \zeta+i_{e_{i}} \zeta, \quad \Gamma_{i+n} \zeta=i\left(e_{i} \wedge \zeta-i_{e_{i}} \zeta\right), \quad i=1, \ldots, n-1,
\end{aligned}
$$

where $\zeta \in \Lambda^{*}\left(\mathbb{C}^{n}\right)$, and $i_{e_{n}}$ and $i_{e_{i}}$ are the inner derivations with respect to the vectors constructed from $e_{n}$ and $e_{i}$. A straightforward computation reveals that the gamma matrices satisfy the Clifford algebra relation $\Gamma_{A} \Gamma_{B}+\Gamma_{B} \Gamma_{A}=2 \mathbf{1} \eta_{A B}$, where $\eta$ is the Lorentzian metric with mostly plus signature.

The Dirac inner product $D$ is defined as

$$
D(\eta, \zeta):=\left\langle\Gamma_{0} \eta, \zeta\right\rangle,
$$

and it can be shown to be $\operatorname{Spin}(2 n-1,1)$ invariant, where $\operatorname{Spin}(2 n-1,1)$ is the component of the spin group connected to identity element. Observe that the standard Hermitian inner product $\langle\cdot, \cdot\rangle$ is not invariant. This inner product is also written as $D(\eta, \zeta)=\bar{\eta} \zeta$, where $\bar{\eta}$ is called the Dirac conjugate of $\eta$. Again the Dirac representation of $\operatorname{Spin}(2 n-1,1)$ is reducible and decomposes as ${ }^{c} \Delta={ }^{c} \Delta^{+} \oplus^{c} \Delta^{-}$into Weyl chiral and anti-chiral representations according to the decomposition of $\Lambda^{*}\left(\mathbb{C}^{n}\right)$ into forms of even and odd degree, respectively.

Next consider the linear maps $a=\prod_{i=1}^{n} \Gamma_{i}$ and $b=\Gamma_{0} \prod_{i=1}^{n-1} \Gamma_{i+n}$. There are two $\operatorname{Spin}(2 n-1,1)$-invariant bi-linears which can be constructed on ${ }^{c} \Delta$ given by

$$
\mathrm{A}(\eta, \zeta)=\left\langle a \eta^{*}, \zeta\right\rangle, \quad \mathrm{B}(\eta, \zeta)=\left\langle b \eta^{*}, \zeta\right\rangle,
$$

where $\eta, \zeta \in{ }^{c} \Delta$. A straightforward computation following the definitions reveals that

$$
\begin{aligned}
& \mathrm{A}\left(\eta, \Gamma_{A} \zeta\right)=(-1)^{n-1} \mathrm{~A}\left(\Gamma_{A} \eta, \zeta\right), \mathrm{A}\left(\eta, \Gamma_{A} \zeta\right)=(-1)^{\frac{(n+2)(n-1)}{2}} \mathrm{~A}\left(\zeta, \Gamma_{A} \eta\right), \\
& \mathrm{B}\left(\eta, \Gamma_{A} \zeta\right)=(-1)^{n} \mathrm{~B}\left(\Gamma_{A} \eta, \zeta\right), \quad \mathrm{B}\left(\eta, \Gamma_{A} \zeta\right)=(-1)^{\frac{n(n-1)}{2}} \mathrm{~B}\left(\zeta, \Gamma_{A} \eta\right) .
\end{aligned}
$$

It is clear that both $A$ and $B$ are invariant under $\operatorname{Spin}(2 n-1,1)$ and in addition that one of them is also invariant under $\operatorname{Pin}(2 n-1,1)$. Also the gamma matrices are either symmetric or skew-symmetric with respect to these bi-linears.

In the Lorentzian case, real representations are constructed by relating the Dirac and Majorana conjugates. So one considers the anti-linear maps $r_{\mathrm{A}}:=\Gamma_{0} a *$ and $r_{\mathrm{B}}:=\Gamma_{0} b *$ and after some straightforward calculation finds that

$$
\begin{aligned}
& r_{\mathrm{A}}^{2}=(-1)^{\frac{(n+2)(n-1)}{2}} \mathbf{1}, \quad r_{\mathrm{A}} \Gamma_{\mathrm{A}}=(-1)^{n} \Gamma_{\mathrm{A}} r_{\mathrm{A}}, \\
& r_{\mathrm{B}}^{2}=(-1)^{\frac{n(n-1)}{2}} \mathbf{1}, \quad r_{\mathrm{B}} \Gamma_{\mathrm{A}}=(-1)^{n-1} \Gamma_{\mathrm{A}} r_{\mathrm{B}} .
\end{aligned}
$$

Imposing a reality condition $\mathcal{R}$ which is proportional to $r_{\mathrm{A}}$ and $r_{\mathrm{B}}$ up to a phase, one finds that $\operatorname{Spin}(2 n-1,1)$ has real representations provided that $\left[\frac{n-1}{2}\right] \in 2 \mathbb{Z}$. Moreover, there are Majorana-Weyl representations provided $n \in 4 \mathbb{Z}+1$. The linear maps $C_{\mathrm{A}}=\Gamma_{0} a$ and $C_{\mathrm{B}}=\Gamma_{0} b$ are called charge conjugation matrices.

The Dirac representation admits an oscillator basis as in the Euclidean case. In particular, one has that

$$
\begin{aligned}
& \gamma_{-}=\frac{1}{\sqrt{2}}\left(\Gamma_{n}-\Gamma_{0}\right)=\sqrt{2} e_{n} \wedge, \gamma_{\alpha}=\frac{1}{\sqrt{2}}\left(\Gamma_{\alpha}-i \Gamma_{\alpha+n}\right)=\sqrt{2} e_{\alpha} \wedge, \\
& \gamma_{+}=\frac{1}{\sqrt{2}}\left(\Gamma_{n}+\Gamma_{0}\right)=\sqrt{2} i_{e_{n}}, \quad \gamma_{\bar{\alpha}}=\frac{1}{\sqrt{2}}\left(\Gamma_{\alpha}+i \Gamma_{\alpha+n}\right)=\sqrt{2} i_{e_{\alpha}}
\end{aligned}
$$

satisfy the Clifford algebra relation $\gamma_{A} \gamma_{B}+\gamma_{B} \gamma_{A}=21 \eta_{A B}$, where now the non-vanishing component of the Lorentzian metric $\eta$ in this basis are $\eta_{+-}=1$ and $\eta_{\alpha \bar{\beta}}=\delta_{\alpha \bar{\beta}}$. It is clear that the whole Dirac representation can be constructed by acting with the "creation operators" $\left(\gamma^{+}, \gamma^{\bar{\alpha}}\right)$ on the Clifford vacuum represented by the 0 -degree form 1 , where $\gamma^{A}=\eta^{A B} \gamma_{B}$. This basis is analogous to the Hermitian basis in the Euclidean case described in the previous appendix. The difference is the two light-cone directions it contains. On curved spaces, there is a (local) spacetime coframe for which the gamma matrices take 
the form (B.12). In the spinorial geometry approach, the solutions to the KSEs are expressed in such a coframe. Because of this, we shall refer to such coframe as a "spinorial geometry coframe".

The construction of spinor representations of $\operatorname{Spin}(2 n, 1)$ can be done in a way similar to the one we have explained for $\operatorname{Spin}(2 n+1)$. Though here for the realization of the Majorana representations of $\operatorname{Spin}(2 n, 1)$ in terms of forms, one can begin from Majorana representations of either $\operatorname{Spin}(2 n)$ or $\operatorname{Spin}(2 n-1,1)$. This has been utilized in the solution of the KSE of $d=11$ supergravity for $N=1$ backgrounds.

\section{Appendix C. Group manifolds, symmetric and homogeneous spaces}

\section{C.1. Homogeneous spaces}

A detailed exposition of the geometry of group manifolds, symmetric and homogeneous spaces can be found in [269]. Here we summarize some basic properties of the latter which have been used throughout the review. Consider the left coset space $G / H$, where $G$ is a Lie group which acts effectively from the left on $G / H$ and $H$ is a closed Lie subgroup of $G$. Let us denote the Lie algebras of $G$ and $H$ with $\mathfrak{g}$ and $\mathfrak{h}$, respectively, and assume that there is a decomposition $\mathfrak{g}=\mathfrak{h} \oplus \mathfrak{m}$ such that

$$
\begin{aligned}
& {\left[h_{\alpha}, h_{\beta}\right]=f_{\alpha \beta}{ }^{\gamma} h_{\gamma}, \quad\left[h_{\alpha}, m_{A}\right]=f_{\alpha A}{ }^{B} m_{B},} \\
& {\left[m_{A}, m_{B}\right]=f_{A B}{ }^{C} m_{C}+f_{A B}{ }^{\alpha} h_{\alpha},}
\end{aligned}
$$

where $h_{\alpha}, \alpha=1,2, \ldots, \operatorname{dim} \mathfrak{h}$ and $m_{A}, A=1, \ldots, \operatorname{dim} \mathfrak{g}-\operatorname{dim} \mathfrak{h}$ are bases in $\mathfrak{h}$ and $\mathfrak{m}$, respectively. If $f_{A B}{ }^{c}=0$, that is $[\mathfrak{m}, \mathfrak{m}] \subseteq \mathfrak{h}, G / H$ will be a symmetric space.

Let $s: U \subset G / H \rightarrow G$ be a local section of the coset. The decomposition of the Maurer-Cartan form in components along $\mathfrak{h}$ and $\mathfrak{m}$ is

$$
s^{-1} d s=\ell^{A} m_{A}+\Psi^{\alpha} h_{\alpha},
$$

which defines a local left-invariant coframe $\ell^{A}$ and a canonical left-invariant connection $\Psi^{\alpha}$ on $G / H$. The curvature and torsion of the canonical connection are

$$
\begin{aligned}
& R^{\alpha}:=d \Psi^{\alpha}+\frac{1}{2} f_{\beta \gamma}{ }^{\alpha} \Psi^{\beta} \wedge \Psi^{\gamma}=-\frac{1}{2} f_{B C}{ }^{\alpha} \ell^{B} \wedge \ell^{C}, \\
& T^{A}:=d \ell^{A}+f_{\beta C}{ }^{A} \Psi^{\beta} \wedge \boldsymbol{\ell}^{C}=-\frac{1}{2} f_{B C}{ }^{A} \ell^{B} \wedge \boldsymbol{\ell}^{C},
\end{aligned}
$$

respectively, where the equalities follow after taking the exterior derivative of (C.2) and using (C.1). If $G / H$ is symmetric, then the torsion vanishes.

Left-invariant metrics $d s^{2}$ and p-forms $\omega$ on $G / H$ can be written as

$$
d s^{2}=g_{A B} \ell^{A} \ell^{B}, \quad \omega=\frac{1}{p !} \omega_{A_{1} \ldots A_{p}} \ell^{A_{1}} \wedge \cdots \wedge \ell^{A_{p}},
$$

respectively, where the components $g_{A B}$ and $\omega_{A_{1} \ldots A_{p}}$ are constant and satisfy

$$
f_{\alpha(A}{ }^{C} g_{B) C}, \quad f_{\alpha\left[A_{1}\right.}{ }^{B} \omega_{\left.A_{2} \ldots A_{p}\right] B}=0 .
$$

The latter condition is required for invariance under the right action of $H$ on $G$. All left-invariant forms are parallel with respect to the canonical connection.

For symmetric spaces, the canonical connection coincides with the Levi-Civita connection of invariant metrics. However for the rest of the homogeneous spaces this is not the case as the canonical connection has non-vanishing torsion. Let $\Omega$ be the Levi-Civita connection of an invariant metric $d s^{2}$ in the left-invariant coframe. As the difference of two connections is a tensor, we set

$$
\Omega^{A}{ }_{B}=\Psi^{\alpha} f_{\alpha B}{ }^{A}+\ell^{C} Q_{C}{ }^{A}{ }_{B} .
$$

Requiring that $\Omega$ is metric and torsion free,

$$
\Omega_{A B}+\Omega_{B A}=0, d \ell^{A}+\Omega_{B}^{A} \wedge \ell^{B}=0,
$$

respectively, one finds that

$$
\Omega^{A}{ }_{B}=\Psi^{\alpha} f_{\alpha B}{ }^{A}+\frac{1}{2}\left(g^{A D} f_{D B}{ }^{E} g_{C E}+g^{A D} f_{D C}{ }^{E} g_{B E}+f_{C B}{ }^{A}\right) \ell^{C} .
$$

In turn, the Riemann curvature 2 -form $R_{B}^{A}$ is

$$
R_{B}^{A}=\frac{1}{2}\left(Q_{C}{ }^{A}{ }_{E} Q_{D}{ }_{B}{ }_{B}-Q_{D}{ }^{A}{ }_{E} Q_{C},{ }_{B}{ }_{B}-Q_{E}{ }^{A}{ }_{B} f_{C D}{ }^{E}-f_{C D}{ }^{\alpha} f_{\alpha B}{ }^{A}\right) \ell^{C} \wedge \boldsymbol{\ell}^{D} .
$$

Note that the expression for $\Omega^{A}{ }_{B}$ is considerably simplified whenever the coset space is naturally reductive because the structure constants $f_{A B C}=f_{A B}{ }^{E} g_{C E}$ are then skew-symmetric. 


\section{C.2. Cahen-Wallach spaces}

Cahen-Wallach spaces $C W_{n}$ are plane-wave spacetimes which are also symmetric spaces. In Brinkmann coordinates, their metric can be written as

$$
d s^{2}=2 d v\left(d u+A_{i j} y^{i} y^{j} d v\right)+\delta_{i j} d y^{i} d y^{j},
$$

where $A$ is a constant matrix.

A subclass of $C W_{n}$ spaces are also group manifolds. To identify these, consider the non-vanishing Lie bracket commutators

$$
\left[t_{i}, t_{j}\right]=-\beta_{i j} t_{+}, \quad\left[t_{-}, t_{i}\right]=\beta^{j}{ }_{i} t_{j},
$$

where $\left\{t_{+}, t_{-}, t_{i}: i=1, \ldots, n-2\right\}$ are some generators and $\left(\beta_{i j}\right)$ is a non-degenerate skew-symmetric matrix, $\beta_{i j}=\delta_{i k} \beta^{k}$. These give rise to the Maurer-Cartan relations

$$
d \ell^{-}=0, \quad d \ell^{i}=-\beta_{j}^{i} \ell^{-} \wedge \ell^{j}, \quad d \ell^{+}=\frac{1}{2} \beta_{i j} \ell^{i} \wedge \ell^{j},
$$

where $\ell=\ell^{-} t_{-}+\ell^{+} t_{+}+\ell^{i} t_{i}$ is a left invariant coframe. These can be solved as

$$
\begin{aligned}
& \ell^{-}=d v, \quad \ell^{i}=d x^{i}+\beta_{j}^{i} x^{j} d v, \\
& \ell^{+}=d u+\beta_{i j} x^{i} d x^{j}-\frac{1}{2} \beta_{k i} \beta^{k} x^{i} x^{j} d v,
\end{aligned}
$$

for some coordinates $\left(u, v, x^{i}\right)$. The most general bi-invariant metric up to an overall scale and a redefinition of $\ell^{+}$as $\ell^{+} \rightarrow \ell^{+}+\lambda \ell^{-}$is

$$
d s^{2}=2 \ell^{-} \ell^{+}+\delta_{i j} \ell^{i} \ell^{j} .
$$

Substituting (C.13) into the metric, and after a further coordinate transformation $y=e^{\frac{1}{2} v \beta} x$, one finds (C.10) for $A_{i j}=$ $-\frac{1}{8} \beta_{k i} \beta^{k}{ }_{j}$. Observe that without loss of generality $\beta$ can be chosen to be in block-diagonal form in which case $A$ becomes a diagonal negative definite matrix. Amongst the $C W_{n}$ spaces that are group manifolds, $C W_{6}$ with self-dual structure constants $\beta$ appears in the description of supersymmetric backgrounds in $d=5$ and $d=6$ supergravities as well as in the heterotic theory.

\section{Appendix D. Fierz identities for $d=5$ supergravity}

Let $\epsilon_{1}, \epsilon_{2}, \epsilon_{3}, \epsilon_{4}$ be Dirac spinors of $\operatorname{Spin}(4,1)$. These satisfy the following Fierz identity

$$
\begin{aligned}
D\left(\epsilon_{1}, \epsilon_{2}\right) D\left(\epsilon_{3}, \epsilon_{4}\right) & =\frac{1}{4} D\left(\epsilon_{1}, \epsilon_{4}\right) D\left(\epsilon_{3}, \epsilon_{2}\right)+\frac{1}{4} D\left(\epsilon_{1}, \Gamma_{A} \epsilon_{4}\right) D\left(\epsilon_{3}, \Gamma^{A} \epsilon_{2}\right) \\
& -\frac{1}{8} D\left(\epsilon_{1}, \Gamma_{A B} \epsilon_{4}\right) D\left(\epsilon_{3}, \Gamma^{A B} \epsilon_{2}\right),
\end{aligned}
$$

where $D$ is the Dirac inner product given in (B.8). This Fierz identity is equivalent to

$$
\begin{aligned}
D\left(\epsilon_{3}, \epsilon_{4}\right) \epsilon_{2} & =\frac{1}{4} D\left(\epsilon_{3}, \epsilon_{2}\right) \epsilon_{4}+\frac{1}{4} D\left(\epsilon_{3}, \Gamma_{A} \epsilon_{2}\right) \Gamma^{A} \epsilon_{4} \\
& -\frac{1}{8} D\left(\epsilon_{3}, \Gamma_{A B} \epsilon_{2}\right) \Gamma^{A B} \epsilon_{4} .
\end{aligned}
$$

These Fierz identities differ from those used in [8], because in that work a mostly minus signature spacetime metric was used, whereas here we use a mostly plus metric. Given a spinor $\epsilon$, we use the Fierz identities to obtain algebraic conditions on the bilinears defined in (101) and (102).

(i) Setting $\epsilon_{1}=\epsilon_{2}=\epsilon_{3}=\epsilon_{4}=\epsilon$ gives

$$
-\frac{3}{4} f^{4}=\frac{1}{4} X^{2}-\frac{1}{8}\left(\omega_{1}\right)^{2}
$$

(ii) Setting $\epsilon_{1}=\epsilon_{4}=r_{\mathrm{A}} \epsilon, \epsilon_{2}=\epsilon_{3}=\epsilon$ gives

$$
\frac{1}{4} f^{4}=\frac{1}{4} X^{2}+\frac{1}{8}\left(\omega_{1}\right)^{2}
$$

(iii) Setting $\epsilon_{1}=\epsilon_{2}=r_{\mathrm{A}} \epsilon, \epsilon_{3}=\epsilon_{4}=\epsilon$ gives

$$
\xi_{A B} \bar{\xi}^{A B}=8 f^{4} \text {. }
$$


(iv) Setting $\epsilon_{1}=\epsilon_{2}=\epsilon_{3}=\epsilon, \epsilon_{4}=r_{\mathrm{A}} \epsilon$ gives

$$
\left(\omega_{1}\right)_{A B} \xi^{A B}=0 .
$$

(v) Setting $\epsilon_{1}=\epsilon_{3}=\epsilon, \epsilon_{2}=\epsilon_{4}=r_{\mathrm{A}} \epsilon$ gives

$$
\xi^{2}=0 \text {. }
$$

In particular, these conditions also imply that

$$
X^{2}=-f^{4},
$$

so the vector bilinear is either null or timelike depending on whether $f=0$ or $f \neq 0$ respectively. Furthermore, we also have

$$
\left(\omega_{1}\right)^{2}=4 f^{4}
$$

In obtaining these expressions we have made use of the identities

$$
D\left(r_{\mathrm{A}} \epsilon_{1}, r_{\mathrm{A}} \epsilon_{2}\right)=D\left(\epsilon_{2}, \epsilon_{1}\right),
$$

and

$$
D\left(\Gamma_{A} \epsilon_{1}, \epsilon_{2}\right)=-D\left(\epsilon_{1}, \Gamma_{A} \epsilon_{2}\right), \quad D\left(\Gamma_{A B} \epsilon_{1}, \epsilon_{2}\right)=-D\left(\epsilon_{1}, \Gamma_{A B} \epsilon_{2}\right),
$$

which imply

$$
D\left(r_{\mathrm{A}} \epsilon, \Gamma_{\mathrm{A}} r_{\mathrm{A}} \epsilon\right)=D\left(\epsilon, \Gamma_{A} \epsilon\right), \quad D\left(r_{\mathrm{A}} \epsilon, \Gamma_{A B} r_{\mathrm{A}} \epsilon\right)=-D\left(\epsilon, \Gamma_{A B} \epsilon\right) .
$$

Further algebraic conditions, which are also useful in determining the various types of projection conditions which the spinors must satisfy are obtained by considering the Fierz identity (D.2). In particular, on setting $\epsilon_{2}=\epsilon_{3}=\epsilon_{4}=\epsilon$, and also $\epsilon_{2}=\epsilon_{3}=r_{\mathrm{A}} \epsilon, \epsilon_{4}=\epsilon$ and comparing the expressions gives

$$
X_{A} \Gamma^{A} \epsilon=i f^{2} \epsilon \text {, }
$$

and

$$
\left(\omega_{1}\right)_{A B} \Gamma^{A B} \epsilon=-4 i f^{2} \epsilon
$$

Also, setting $\epsilon_{3}=\epsilon_{4}=\epsilon, \epsilon_{2}=r_{\mathrm{A}} \epsilon$ gives

$$
\xi_{A B} \Gamma^{A B} \epsilon=-8 i f^{2} \epsilon,
$$

and setting $\epsilon_{3}=\epsilon, \epsilon_{2}=\epsilon_{4}=r_{\mathrm{A}} \epsilon$ leads to

$$
\bar{\xi}_{A B} \Gamma^{A B} \epsilon=0 \text {. }
$$

The condition (D.14) implies that

$$
D\left(\epsilon, \Gamma_{L}\left(\omega_{1}\right)_{A B} \Gamma^{A B} \epsilon\right)=D\left(\epsilon,-4 i f^{2} \Gamma_{L} \epsilon\right),
$$

which on taking real and imaginary parts gives

$$
i_{X} \omega_{1}=0,
$$

and

$$
\left(\omega_{1}\right)_{A B}{ }^{*}\left(\omega_{1}\right)_{C}^{A B}=4 f^{2} X_{C} .
$$

Similarly, (D.15) and (D.16) lead to

$$
i_{X} \xi=0, \quad \xi_{A B}{ }^{*}\left(\omega_{1}\right)_{C}{ }^{A B}=0, \quad \xi_{A B}{ }^{*} \bar{\xi}_{C}{ }^{A B}=8 f^{2} X_{C}, \quad \xi_{A B}{ }^{*} \xi_{C}{ }^{A B}=0 .
$$

Next, note that (D.13) implies that

$$
D\left(\epsilon, \Gamma_{A B} X_{C} \Gamma^{C} \epsilon\right)=i f^{2}\left(\omega_{1}\right)_{A B}, \quad D\left(\epsilon, \Gamma_{A B} X_{C} \Gamma^{C} r_{A} \epsilon\right)=i f^{2} \xi_{A B},
$$

which in turn gives that

$$
i_{X}^{*}\left(\omega_{1}\right)_{A B}=-f^{2}\left(\omega_{1}\right)_{A B}, \quad i_{X}^{*} \xi_{A B}=-f^{2} \xi_{A B} .
$$

Additional bilinear identities are then obtained from (D.1) on setting

(a) Setting $\epsilon_{1}=\epsilon_{3}=\epsilon, \epsilon_{2}=\Gamma_{B} \epsilon, \epsilon_{4}=\Gamma_{A} \epsilon$ gives

$$
\left(\omega_{1}\right)_{C A}\left(\omega_{1}\right)^{C}{ }_{B}=X_{A} X_{B}+f^{4} g_{A B} \text {. }
$$


(b) Setting $\epsilon_{1}=\epsilon_{3}=\epsilon, \epsilon_{2}=\Gamma_{B} r_{A} \epsilon, \epsilon_{4}=\Gamma_{A} \epsilon$ gives

$$
\left(\omega_{1}\right)_{C(A} \xi_{B)}^{C}=0 .
$$

Furthermore, the identity (D.14) implies that

$$
D\left(\epsilon, \Gamma_{A B}\left(\omega_{1}\right)_{C D} \Gamma^{C D} r_{A} \epsilon\right)=4 i f^{2} \Gamma_{A B} r_{A} \epsilon,
$$

which in turn gives

$$
\left(\omega_{1}\right)_{B C} \xi_{A}{ }^{C}-\left(\omega_{1}\right)_{A C} \xi_{B}{ }^{C}=2 i f^{2} \xi_{A B} .
$$

It follows that

$$
\left(\omega_{1}\right)_{B C} \xi_{A}{ }^{C}=i f^{2} \xi_{A B} .
$$

(c) Setting $\epsilon_{1}=\epsilon_{3}=\epsilon, \epsilon_{2}=\Gamma_{B} r_{A} \epsilon, \epsilon_{4}=\Gamma_{A} r_{A} \epsilon$ gives

$$
\xi_{C A} \xi^{C}{ }_{B}=0,
$$

on using (D.7).

(d) Setting $\epsilon=r_{\mathrm{A}} \epsilon, \epsilon_{2}=\Gamma_{B} r_{\mathrm{A}} \epsilon, \epsilon_{3}=\epsilon, \epsilon_{4}=\Gamma_{A} \epsilon$, one finds

$$
\bar{\xi}_{C A} \xi_{B}^{C}+\bar{\xi}_{C B} \xi_{A}^{C}=4 X_{A} X_{B}+4 f^{4} g_{A B} \text {. }
$$

Also, the condition (D.15) implies that

$$
D\left(r_{A} \epsilon, \Gamma_{A B} \xi_{C D} \Gamma^{C D} \epsilon\right)=-8 i f^{2} D\left(r_{A} \epsilon, \Gamma_{A B} r_{A} \epsilon\right),
$$

and hence

$$
\bar{\xi}_{A}{ }^{C} \xi_{B C}-\bar{\xi}_{B}{ }^{C} \xi_{A C}=4 i f^{2}\left(\omega_{1}\right)_{A B} .
$$

It follows that

$$
\bar{\xi}_{C A} \xi_{B}^{C}=2 i f^{2}\left(\omega_{1}\right)_{A B}+2 X_{A} X_{B}+2 f^{4} g_{A B} .
$$

This completes the Fierz identities needed to solve the KSEs of $\mathcal{N}=1 d=5$ supergravity.

\section{Appendix E. $d=11$ and type II $d=10$ supergravities}

Here we summarize key properties of $d=11$ and IIB $d=10$ supergravities that we are using throughout the review. Some additional formulae which include the integrability conditions of the KSEs are also given.

\section{E.1. $d=11$ supergravity}

The action of the bosonic fields of $d=11$ supergravity [174] is

$$
I=\int_{M}\left(\frac{1}{2} R \mathrm{dvol}+\frac{1}{4} F \wedge \star F+\frac{1}{12} F \wedge F \wedge A\right),
$$

where $F=d A, A$ is the 3 -form gauge potential, $R$ is the scalar curvature of the metric $g$ and dvol is the spacetime volume form. For a superspace formulation see [270].

The KSE of $d=11$ supergravity has already been given in (275) and the supercovariant derivative $\mathcal{D}$ has been presented in (276). The supersymmetry parameter $\epsilon$ is in the 32-dimensional Majorana representation $\Delta_{32}$ of $\operatorname{Spin}(10,1)$.

The integrability condition of the gravitino KSE is

$$
\left[\mathcal{D}_{M}, \mathcal{D}_{N}\right] \epsilon \equiv \mathcal{R}_{M N} \epsilon=0,
$$

where $\mathcal{R}$ is the curvature of the supercovariant connection [10]

$$
\begin{aligned}
& \mathcal{R}_{M N}=\frac{1}{4} R_{M N, A B} \Gamma^{A B}+\frac{2}{(288)^{2}} F_{A_{1} \ldots A_{4}} F_{B_{1} \ldots B_{4}} \epsilon_{M N}{ }^{A_{1} \ldots A_{4} B_{1} \ldots B_{4}}{ }_{C} \Gamma^{C} \\
& +\frac{48}{(288)^{2}}\left[4 F_{M A_{1} A_{2} A_{3}} F^{A_{1} A_{2} A_{3}}{ }_{B} \Gamma^{B}{ }_{N}-4 F_{N A_{1} A_{2} A_{3}} F^{A_{1} A_{2} A_{3}}{ }_{B} \Gamma^{B}{ }_{M}\right. \\
& \left.-36 F_{A B M C} F^{A B}{ }_{N D} \Gamma^{C D}+F_{A_{1} \ldots A_{4}} F^{A_{1} \ldots A_{4}} \Gamma_{M N}\right] \\
& +\frac{1}{36}\left[\nabla_{M} F_{N A_{1} A_{2} A_{3}}-\nabla_{N} F_{M A_{1} A_{2} A_{3}}\right] \Gamma^{A_{1} A_{2} A_{3}} \\
& -\frac{8}{(288)^{23} 3}\left[F_{B_{1} \ldots B_{4}} F_{C_{1} C_{2} C_{3} N} \epsilon_{M}{ }^{B_{1} \ldots B_{4} C_{1} C_{2} C_{3}}{ }_{A_{1} A_{2} A_{3}}-(N \leftrightarrow M)\right] \Gamma^{A_{1} A_{2} A_{3}} \\
& -\frac{1}{432}\left[4 F_{\mathrm{CA}_{1} A_{2} A_{3}} F^{\mathrm{C}}{ }_{{ }_{M N A_{4}}} \Gamma^{A_{1} \ldots A_{4}}\right. \\
& \left.+3 F_{B C A_{1} A_{2}} F^{B C A_{3}}{ }_{N} \Gamma^{A_{1} A_{2}}{ }_{{ }_{M A_{3}}}-3 F_{B C A_{1} A_{2}} F^{B C A_{3}}{ }_{M} \Gamma^{A_{1} A_{2}}{ }_{N A_{3}}\right]
\end{aligned}
$$




$$
\begin{aligned}
& -\frac{1}{288}\left[\nabla_{M} F_{A_{1} \ldots A_{4}} \Gamma^{A_{1} \ldots A_{4}}{ }_{N}-(N \leftrightarrow M)\right] \\
& -\frac{1}{(72)^{2} 5 !}\left[-6 F_{M B_{1} B_{2} B_{3}} F_{N C_{1} C_{2} C_{3}} \epsilon^{B_{1} B_{2} B_{3} C_{1} C_{2} C_{3} C_{A_{1} \ldots A_{5}}}\right. \\
& -6 F_{M P B_{1} B_{2}} F^{P} C_{1} C_{2} C_{3} \epsilon_{N}{ }^{B_{1} B_{2} C_{1} C_{2} C_{3}{ }_{3} \ldots A_{1} \ldots A_{5}} \\
& +6 F_{N B_{1} B_{2}} F^{P} C_{1} C_{2} C_{3} \epsilon_{M}{ }^{B_{1} B_{2} C_{1} C_{2} C_{3}}{ }_{A_{1} \ldots A_{5}} \\
& \left.+9 F_{L P B_{1} B_{2}} F^{L P}{ }_{C_{1} C_{2}} \epsilon_{M N}{ }^{B_{1} B_{2} C_{1} C_{2}{ }_{A_{1} \ldots} \ldots A_{5}}\right] \Gamma^{A_{1} \ldots A_{5}},
\end{aligned}
$$

and where we have used that

$$
\Gamma^{A_{1} \ldots A_{2 k}}=-\frac{(-1)^{k}}{(11-2 k) !} \epsilon^{A_{1} \ldots A_{2 k}{ }_{B_{1} \ldots B_{11}-2 k}} \Gamma^{B_{1} \ldots B_{11-2 k}},
$$

with $\epsilon_{01 \ldots 9 \natural}=-1$.

One expects that $\Gamma^{B} \mathcal{R}_{A B}$ can be expressed in terms of the field equations and Bianchi identities as it arises from the supersymmetry variation of the gravitino field equation. A direct computation reveals [185] that

$$
\begin{gathered}
\Gamma^{B} \mathcal{R}_{A B}=E_{A B} \Gamma^{B}-\frac{1}{36} L F_{C_{1} C_{2} C_{3}}\left(\Gamma_{A}{ }^{C_{1} C_{2} C_{3}}-6 \delta_{A}^{C_{1}} \Gamma^{C_{2} C_{3}}\right)+ \\
+\frac{1}{6 !} B F_{C_{1} \ldots C_{5}}\left(\Gamma_{A}{ }^{C_{1} \cdots C_{5}}-10 \delta_{A}^{C_{1}} \Gamma^{C_{2} \cdots C_{5}}\right),
\end{gathered}
$$

where

$$
\begin{aligned}
E_{A B} & :=R_{A B}-\frac{1}{12} F_{A C_{1} C_{2} C_{3}} F_{B}{ }^{C_{1} C_{2} C_{3}}+\frac{1}{144} g_{A B} F_{C_{1} \cdots C_{4}} F^{C_{1} \cdots C_{4}}, \\
L F_{A B C} & :=*\left(d * F+\frac{1}{2} F \wedge F\right)_{A B C},
\end{aligned}
$$

are the Einstein and 4-form flux field equations, respectively, and

$$
B F_{A_{1} \ldots A_{5}}:=(d F)_{A_{1} \ldots A_{5}},
$$

is the Bianchi identity of $F$. Clearly, this vanishes provided that $F$ is closed which is the case in the context of $d=11$ supergravity.

\section{E.2. IIB supergravity}

The bosonic fields of IIB supergravity [192,193] are the spacetime metric $g$, two real scalars, the axion $\sigma$ and the dilaton $\phi$, which are combined into a complex 1-form field strength $P$, two 3 -form field strengths $G^{1}$ and $G^{2}$ which are combined to a complex 3-form field strength $G$, and a self-dual 5-form field strength $F$. To describe these, we introduce a $S U(1,1)$ matrix $U=\left(V_{+}^{a}, V_{-}^{a}\right), a=1,2$ such that

$$
V_{-}^{a} V_{+}^{b}-V_{-}^{b} V_{+}^{a}=\epsilon^{a b},
$$

where $\epsilon^{12}=1=\epsilon_{12},\left(V_{-}^{1}\right)^{*}=V_{+}^{2}$ and $\left(V_{-}^{2}\right)^{*}=V_{+}^{1}$. The signs denote $U(1) \subset S U(1,1)$ charge. Then set

$$
P_{M}:=-\epsilon_{a b} V_{+}^{a} \partial_{M} V_{+}^{b}, \quad Q_{M}:=-i \epsilon_{a b} V_{-}^{a} \partial_{M} V_{+}^{b} .
$$

The 3-form field strengths $G_{M N R}^{a}=3 \partial_{[M} A_{N R}^{a}$, with $\left(A_{M N}^{1}\right)^{*}=A_{M N}^{2}$ combine into the complex field strength

$$
G_{M N R}:=-\epsilon_{a b} V_{+}^{a} G_{M N R}^{b} .
$$

The five-form self-dual field strength is

$$
F_{M_{1} M_{2} M_{3} M_{4} M_{5}}:=5 \partial_{\left[M_{1}\right.} A_{\left.M_{2} M_{3} M_{4} M_{5}\right]}+\frac{5 i}{8} \epsilon_{a b} A_{\left[M_{1} M_{2}\right.}^{a} G_{\left.M_{3} M_{4} M_{5}\right]}^{b},
$$

where $F_{M_{1} \ldots M_{5}}=\frac{1}{5 !} \epsilon_{M_{1} \ldots M_{5}}{ }^{N_{1} \ldots N_{5}} F_{N_{1} \ldots N_{5}}$ and $\epsilon_{01 \ldots 9}=-1$. The axion $\sigma$ and the dilaton $\phi$ fields can be combined into a complex scalar, $\tau:=\sigma+i e^{-\phi}$. In turn this is related to $V$ as

$$
\frac{V_{-}^{2}}{V_{-}^{1}}=\frac{1+i \tau}{1-i \tau}
$$

This completes the description of the bosonic fields of the theory.

The KSEs of IIB supergravity are the gravitino KSE which is the parallel transport equation

$$
\mathcal{D} \epsilon=0,
$$

of the supercovariant connection

$$
\mathcal{D}_{M}:=\tilde{\nabla}_{M}+\frac{i}{48} \Gamma^{N_{1} \ldots N_{4}} F_{N_{1} \ldots N_{4} M}-\frac{1}{96}\left(\Gamma_{M}^{N_{1} N_{2} N_{3}} G_{N_{1} N_{2} N_{3}}-9 \Gamma^{N_{1} N_{2}} G_{M N_{1} N_{2}}\right) C *,
$$


and the algebraic KSE

$$
\mathcal{A} \epsilon:=P_{M} \Gamma^{M} C \epsilon^{*}+\frac{1}{24} G_{N_{1} N_{2} N_{3}} \Gamma^{N_{1} N_{2} N_{3}} \epsilon=0,
$$

where

$$
\tilde{\nabla}_{M}=D_{M}+\frac{1}{4} \Omega_{M, A B} \Gamma^{A B}, \quad D_{M}=\partial_{M}-\frac{i}{2} Q_{M},
$$

is the spin connection, $\nabla_{M}=\partial_{M}+\frac{1}{4} \Omega_{M, A B} \Gamma^{A B}$, twisted with the $U(1)$ connection $Q_{M}, Q_{M}^{*}=Q_{M}$. The supersymmetry parameter, $\epsilon$, is a complex Weyl spinor, $\Gamma_{0 . . .9} \epsilon=\epsilon$, and $C$ is a charge conjugation matrix.

The integrability conditions of the KSEs are

$$
\left[\mathcal{D}_{M}, \mathcal{D}_{N}\right] \epsilon=\mathcal{R}_{M N} \epsilon=0,
$$

and

$$
\left[\mathcal{D}_{M}, \mathcal{A}\right] \epsilon=0,
$$

where $\mathcal{R}$ is the supercovariant curvature given in [14]. The components $\mathcal{I}_{A}=\frac{1}{2} \Gamma_{A}{ }^{B C} \mathcal{R}_{B C}$ and $\mathcal{I}=\Gamma^{M}\left[\mathcal{D}_{M}, \mathcal{A}\right]$ of the integrability conditions can be expressed in terms of field equations and Bianchi identities [188] as

$$
\begin{aligned}
\mathcal{I}_{A} \epsilon=[ & \left.\frac{1}{2} \Gamma^{B} E_{A B}-i \Gamma^{B_{1} B_{2} B_{3}} L F_{A B_{1} B_{2} B_{3}}\right] \epsilon \\
& -\left[\Gamma^{B} L G_{A B}-\Gamma_{A}^{\left.{ }^{B_{1} \ldots B_{4}} B G_{B_{1} \ldots B_{4}}\right] C \epsilon^{*},}\right.
\end{aligned}
$$

and similarly as

$$
\mathcal{I} \epsilon=\left[\frac{1}{2} \Gamma^{A B} L G_{A B}+\Gamma^{A_{1} \ldots A_{4}} B G_{A_{1} \ldots A_{4}}\right] \epsilon+\left[L P+\Gamma^{A B} B P_{A B}\right] C \epsilon^{*},
$$

where

$$
\begin{aligned}
& E_{A B}:=R_{A B}-\frac{1}{2} g_{A B} R-\frac{1}{6} F_{A C_{1} \ldots C_{4}} F_{B}{ }^{C_{1} \ldots C_{4}}-\frac{1}{4} G_{(A}{ }^{C_{1} C_{2}} G_{B) C_{1} C_{2}}^{*} \\
& +\frac{1}{24} g_{A B} G^{C_{1} C_{2} C_{3}} G_{C_{1} C_{2} C_{3}}^{*}-2 P_{(A} P_{B)}^{*}+g_{A B} P^{C} P_{C}^{*} \text {, } \\
& L G_{A B}:=\frac{1}{4}\left(\tilde{\nabla}^{C} G_{A B C}-P^{C} G_{A B C}^{*}+\frac{2 i}{3} F_{A B C_{1} C_{2} C_{3}} G^{C_{1} C_{2} C_{3}}\right) \text {, } \\
& L P:=\tilde{\nabla}^{A} P_{A}+\frac{1}{24} G_{A_{1} A_{2} A_{3}} G^{A_{1} A_{2} A_{3}}, \\
& L F_{A_{1} \ldots A_{4}}:=\frac{1}{3 !}\left(\nabla^{B} F_{A_{1} \ldots A_{4} B}-\frac{i}{288} \epsilon_{A_{1} \ldots A_{4}}{ }^{B_{1} \ldots B_{6}} G_{B_{1} B_{2} B_{3}} G_{B_{4} B_{5} B_{6}}^{*}\right) \text {, } \\
& B F_{A_{1} \ldots A_{6}}:=\frac{1}{5 !}\left(\partial_{\left[A_{1}\right.} F_{\left.A_{2} \ldots A_{6}\right]}-\frac{5 i}{12} G_{\left[A_{1} A_{2} A_{3}\right.} G_{\left.A_{4} A_{5} A_{6}\right]}^{*}\right), \\
& B G_{A_{1} \ldots A_{4}}:=\frac{1}{4 !}\left(D_{\left[A_{1}\right.} G_{\left.A_{2} A_{3} A_{4}\right]}+P_{\left[A_{1}\right.} G_{\left.A_{2} A_{3} A_{4}\right]}^{*}\right) \text {, } \\
& B P_{A B}:=D_{[A} P_{B]} \cdot
\end{aligned}
$$

One can show that $L F$ and $B F$ are not independent but are related by the self-duality condition on $F$. The field strengths $P$ and $G$ have different $U(1) \subset S U(1,1)$ charges. In particular, one has $D_{M} P_{N}:=\partial_{M} P_{N}-2 i Q_{M} P_{N}$ and $D_{M} G_{N_{1} N_{2} N_{3}}:=$ $\partial_{M} G_{N_{1} N_{2} N_{3}}-i Q_{M} G_{N_{1} N_{2} N_{3}}$. This concludes the description of the KSEs, field equations and Bianchi identities of the theory.

\section{References}

[1] P.K. Townsend, The eleven-dimensional supermembrane revisited, Phys. Lett. B 350 (1995) 184-187, 265 (1995), http://dx.doi.org/10.1016/03702693(95)00397-4, arXiv:hep-th/9501068.

[2] C.M. Hull, P.K. Townsend, Unity of superstring dualities, Nuclear Phys. B 438 (1995) 109-137, 236 (1994), http://dx.doi.org/10.1016/0550-3213(94) 00559-W, arXiv:hep-th/9410167.

[3] A. Strominger, C. Vafa, Microscopic origin of the Bekenstein-Hawking entropy, Phys. Lett. B 379 (1996) 99-104, http://dx.doi.org/10.1016/03702693(96)00345-0, arXiv:hep-th/9601029.

[4] J.M. Maldacena, The large N limit of superconformal field theories and supergravity, Internat. J. Theoret. Phys. 38 (1999) 1113-1133, http://dx.doi. org/10.1023/A:1026654312961,10.4310/ATMP.1998.v2.n2.a1, arXiv:hep-th/9711200.

[5] M.J. Duff, J.X. Lu, Black and super p-branes in diverse dimensions, in: 7th Summer School Jorge Andre Swieca: Particles and Fields Sao Paulo, Brazil, January 10-23, 1993, Nuclear Phys. B 416 (1994) 301-334, http://dx.doi.org/10.1016/0550-3213(94)90586-X, arXiv:hep-th/9306052.

[6] K.S. Stelle, BPS branes in supergravity, in: High-Energy Physics and Cosmology. Proceedings, Summer School, Trieste, Italy, June 2-July 4, 1997, 1998, arXiv:hep-th/9803116.

[7] K.P. Tod, All metrics admitting supercovariantly constant spinors, Phys. Lett. 121B (1983) 241-244, http://dx.doi.org/10.1016/0370-2693(83)907979.

[8] J.P. Gauntlett, J.B. Gutowski, C.M. Hull, S. Pakis, H.S. Reall, All supersymmetric solutions of minimal supergravity in five- dimensions, Classical Quantum Gravity 20 (2003) 4587-4634, http://dx.doi.org/10.1088/0264-9381/20/21/005, arXiv:hep-th/0209114.

[9] J. Gillard, U. Gran, G. Papadopoulos, The spinorial geometry of supersymmetric backgrounds, Classical Quantum Gravity 22 (2005) 1033-1076, http://dx.doi.org/10.1088/0264-9381/22/6/009, arXiv:hep-th/0410155.

[10] J.M. Figueroa-O'Farrill, G. Papadopoulos, Maximally supersymmetric solutions of ten-dimensional and eleven-dimensional supergravities, J. High Energy Phys. 03 (2003) 048, http://dx.doi.org/10.1088/1126-6708/2003/03/048, arXiv:hep-th/0211089. 
[11] C. Hull, Holonomy and symmetry in M theory, 2003, arXiv:hep-th/0305039.

[12] M.J. Duff, J.T. Liu, Hidden space-time symmetries and generalized holonomy in M theory, Nuclear Phys. B 674 (2003) 217-230, http://dx.doi.org/10. 1016/j.nuclphysb.2003.09.019, arXiv:hep-th/0303140.

[13] G. Papadopoulos, D. Tsimpis, The holonomy of the supercovariant connection and killing spinors, J. High Energy Phys. 07 (2003) 018, http: //dx.doi.org/10.1088/1126-6708/2003/07/018, arXiv:hep-th/0306117.

[14] G. Papadopoulos, D. Tsimpis, The holonomy of IIB supercovariant connection, Classical Quantum Gravity 20 (2003) L253, http://dx.doi.org/10.1088/ 0264-9381/20/20/103, arXiv:hep-th/0307127.

[15] A. Batrachenko, W.Y. Wen, Generalized holonomy of supergravities with 8 real supercharges, Nuclear Phys. B 690 (2004) 331-340, http://dx.doi. org/10.1016/j.nuclphysb.2004.05.022, arXiv:hep-th/0402141.

[16] S.D. Majumdar, A class of exact solutions of Einstein's field equations, Phys. Rev. 72 (1947) 390-398, http://dx.doi.org/10.1103/PhysRev.72.390.

[17] A. Papapetrou, On static, axially symmetric Einstein-Maxwell fields. Part I, Gen. Relativity Gravitation 8 (1977) 421-427, http://dx.doi.org/10.1007/ BF00765933.

[18] G.W. Gibbons, C.M. Hull, A Bogomolny bound for general relativity and solitons in N=2 supergravity, Phys. Lett. 109B (1982) 190-194, http: //dx.doi.org/10.1016/0370-2693(82)90751-1.

[19] W. Israel, G.A. Wilson, A class of stationary electromagnetic vacuum fields, J. Math. Phys. 13 (1972) 865-871, http://dx.doi.org/10.1063/1.1666066.

[20] Z. Perjes, Solutions of the coupled Einstein Maxwell equations representing the fields of spinning sources, Phys. Rev. Lett. 27 (1971) 1668, http: //dx.doi.org/10.1103/PhysRevLett.27.1668.

[21] M. Cahen, N. Wallach, Lorentzian symmetric spaces, Bull. Am. Math. 76 (1970) 585-591, http://dx.doi.org/10.1016/0370-2693(84)90669-5.

[22] S.W. Hawking, G.F.R. Ellis, The Large Scale Structure of Space-Time, in: Cambridge Monographs on Mathematical Physics, Cambridge University Press, 2011, http://dx.doi.org/10.1017/CB09780511524646.

[23] K.P. Tod, More on supercovariantly constant spinors, Classical Quantum Gravity 12 (1995) 1801-1820, http://dx.doi.org/10.1088/0264-9381/12/7/ 020.

[24] V.A. Kostelecky, M.J. Perry, Solitonic black holes in gauged N=2 supergravity, Phys. Lett. B 371 (1996) 191-198, http://dx.doi.org/10.1016/03702693(95)01607-4, arXiv:hep-th/9512222.

[25] M.M. Caldarelli, D. Klemm, All supersymmetric solutions of N=2, D = 4 gauged supergravity, J. High Energy Phys. 09 (2003) 019, http://dx.doi.org/ 10.1088/1126-6708/2003/09/019, arXiv:hep-th/0307022.

[26] D. Klemm, M. Nozawa, Supersymmetry of the C-metric and the general Plebanski-Demianski solution, J. High Energy Phys. 05 (2013) 123, http: //dx.doi.org/10.1007/JHEP05(2013)123, arXiv:1303.3119.

[27] J.F. Plebanski, M. Demianski, Rotating, charged, and uniformly accelerating mass in general relativity, Ann. Physics 98 (1976) 98-127, http: //dx.doi.org/10.1016/0003-4916(76)90240-2.

[28] S.L. Cacciatori, M.M. Caldarelli, D. Klemm, D.S. Mansi, More on BPS solutions of N = 2, D = 4 gauged supergravity, J. High Energy Phys. 07 (2004) 061, http://dx.doi.org/10.1088/1126-6708/2004/07/061, arXiv:hep-th/0406238.

[29] J. Grover, J.B. Gutowski, W.A. Sabra, Maximally minimal preons in four dimensions, Classical Quantum Gravity 24 (2007) 3259-3270, http://dx.doi. org/10.1088/0264-9381/24/13/008, arXiv:hep-th/0610128.

[30] S.L. Cacciatori, M.M. Caldarelli, D. Klemm, D.S. Mansi, D. Roest, Geometry of four-dimensional Killing spinors, J. High Energy Phys. 07 (2007) 046, http://dx.doi.org/10.1088/1126-6708/2007/07/046, arXiv:0704.0247.

[31] K. Behrndt, D. Lust, W.A. Sabra, Stationary solutions of N=2 supergravity, Nuclear Phys. B 510 (1998) 264-288, http://dx.doi.org/10.1016/S05503213(98)81014-6, arXiv:hep-th/9705169.

[32] P. Meessen, T. Ortin, The supersymmetric configurations of N=2, D=4 supergravity coupled to vector supermultiplets, Nuclear Phys. B 749 (2006) 291-324, http://dx.doi.org/10.1016/j.nuclphysb.2006.05.025, arXiv:hep-th/0603099.

[33] W.A. Sabra, Anti-de sitter BPS black holes in N=2 gauged supergravity, Phys. Lett. B 458 (1999) 36-42, http://dx.doi.org/10.1016/S0370-2693(99) 00564-X, arXiv:hep-th/9903143.

[34] A.H. Chamseddine, W.A. Sabra, Magnetic and dyonic black holes in D = 4 gauged supergravity, Phys. Lett. B 485 (2000) 301-307, http://dx.doi.org/ 10.1016/S0370-2693(00)00652-3, arXiv:hep-th/0003213.

[35] Z.W. Chong, M. Cvetic, H. Lu, C.N. Pope, Charged rotating black holes in four-dimensional gauged and ungauged supergravities, Nuclear Phys. B 717 (2005) 246-271, http://dx.doi.org/10.1016/j.nuclphysb.2005.03.034, arXiv:hep-th/0411045.

[36] S.L. Cacciatori, D. Klemm, D.S. Mansi, E. Zorzan, All timelike supersymmetric solutions of $\mathrm{N}=2$, $\mathrm{D}=4$ gauged supergravity coupled to abelian vector multiplets, J. High Energy Phys. 05 (2008) 097, http://dx.doi.org/10.1088/1126-6708/2008/05/097, arXiv:0804.0009.

[37] D. Klemm, E. Zorzan, All null supersymmetric backgrounds of $N=2$, $D=4$ gauged supergravity coupled to abelian vector multiplets, Classical Quantum Gravity 26 (2009) 145018, http://dx.doi.org/10.1088/0264-9381/26/14/145018, arXiv:0902.4186.

[38] D. Klemm, Rotating BPS black holes in matter-coupled AdS $_{4}$ supergravity, J. High Energy Phys. 07 (2011) 019, http://dx.doi.org/10.1007/JHEP07(2011) 019, arXiv: 1103.4699.

[39] M. Colleoni, D. Klemm, Nut-charged black holes in matter-coupled N=2, D=4 gauged supergravity, Phys. Rev. D 85 (2012) 126003, http://dx.doi.org/ 10.1103/PhysRevD.85.126003, arXiv:1203.6179.

[40] A. Gnecchi, K. Hristov, D. Klemm, C. Toldo, O. Vaughan, Rotating black holes in 4d gauged supergravity, J. High Energy Phys. 01 (2014) 127, http://dx.doi.org/10.1007/JHEP01(2014)127, arXiv:1311.1795.

[41] D.D.K. Chow, G. Compere, Dyonic AdS black holes in maximal gauged supergravity, Phys. Rev. D 89 (6) (2014) 065003, http://dx.doi.org/10.1103/ PhysRevD.89.065003, arXiv:1311.1204.

[42] D. Klemm, E. Zorzan, The timelike half-supersymmetric backgrounds of $\mathrm{N}=2$, D =4 supergravity with Fayet-lliopoulos gauging, Phys. Rev. D 82 (2010) 045012, http://dx.doi.org/10.1103/PhysRevD.82.045012, arXiv:1003.2974.

[43] M. Huebscher, P. Meessen, T. Ortin, S. Vaula, N=2 Einstein-Yang-Mills's BPS solutions, J. High Energy Phys. 09 (2008) 099, http://dx.doi.org/10.1088/ 1126-6708/2008/09/099, arXiv:0806.1477.

[44] M. Huebscher, P. Meessen, T. Ortin, Supersymmetric solutions of N=2 D=4 SUGRA: The whole ungauged shebang, Nuclear Phys. B 759(2006) 228-248, http://dx.doi.org/10.1016/j.nuclphysb.2006.10.004, arXiv:hep-th/0606281.

[45] P. Meessen, T. Ortin, Supersymmetric solutions to gauged N=2 d=4 SUGRA: the full timelike shebang, Nuclear Phys. B 863 (2012) 65-89, http: //dx.doi.org/10.1016/j.nuclphysb.2012.05.023, arXiv:1204.0493.

[46] K. Hristov, H. Looyestijn, S. Vandoren, BPS black holes in N=2 D=4 gauged supergravities, J. High Energy Phys. 08 (2010) 103, http://dx.doi.org/10. 1007/JHEP08(2010)103, arXiv:1005.3650.

[47] G. Lopes Cardoso, B. de Wit, J. Kappeli, T. Mohaupt, Stationary BPS solutions in N=2 supergravity with $R^{2}$ interactions, J. High Energy Phys. 12 (2000) 019, http://dx.doi.org/10.1088/1126-6708/2000/12/019, arXiv:hep-th/0009234.

[48] J. Bellorin, T. Ortin, All the supersymmetric configurations of N=4, d=4 supergravity, Nuclear Phys. B 726 (2005) 171-209, http://dx.doi.org/10.1016/ j.nuclphysb.2005.07.020, arXiv:hep-th/0506056.

[49] P. Meessen, T. Ortin, S. Vaula, All the timelike supersymmetric solutions of all ungauged d=4 supergravities, J. High Energy Phys. 11 (2010) 072 , http://dx.doi.org/10.1007/JHEP11(2010)072, arXiv:1006.0239. 
[50] M. Dunajski, J. Gutowski, W. Sabra, P. Tod, Cosmological Einstein-Maxwell instantons and euclidean supersymmetry: Anti-self-dual solutions, Classical Quantum Gravity 28 (2011) 025007, http://dx.doi.org/10.1088/0264-9381/28/2/025007, arXiv:1006.5149.

[51] M. Dunajski, J.B. Gutowski, W.A. Sabra, P. Tod, Cosmological Einstein-Maxwell instantons and euclidean supersymmetry: Beyond self-duality, J. High Energy Phys. 03 (2011) 131, http://dx.doi.org/10.1007/JHEP03(2011)131, arXiv:1012.1326.

[52] D. Klemm, M. Nozawa, Geometry of killing spinors in neutral signature, Classical Quantum Gravity 32 (18)(2015) 185012, http://dx.doi.org/10.1088/ 0264-9381/32/18/185012, arXiv:1504.02710.

[53] U. Gran, J. Gutowski, G. Papadopoulos, Geometry of all supersymmetric four-dimensional N = 1 supergravity backgrounds, J. High Energy Phys. 06 (2008) 102, http://dx.doi.org/10.1088/1126-6708/2008/06/102, arXiv:0802.1779.

[54] T. Ortin, The supersymmetric solutions and extensions of ungauged matter-coupled N=1, d=4 supergravity, J. High Energy Phys. 05 (2008) 034, http://dx.doi.org/10.1088/1126-6708/2008/05/034, arXiv:0802.1799.

[55] J. Wess, J. Bagger, Supersymmetry and Supergravity, Princeton University Press, 1992,

[56] B.R. Greene, A.D. Shapere, C. Vafa, S.-T. Yau, Stringy cosmic strings and noncompact Calabi-Yau manifolds, Nuclear Phys. B 337 (1990) 1-36, http://dx.doi.org/10.1016/0550-3213(90)90248-C.

[57] J. Gutowski, G. Papadopoulos, Magnetic cosmic strings of N=1, D = 4 supergravity with cosmological constant, Phys. Lett. B 514 (2001) 371-376, http://dx.doi.org/10.1016/S0370-2693(01)00800-0, arXiv:hep-th/0102165.

[58] G. Dvali, R. Kallosh, A. Van Proeyen, D term strings, J. High Energy Phys. 01 (2004) 035, http://dx.doi.org/10.1088/1126-6708/2004/01/035, arXiv: hep-th/0312005.

[59] M. Cvetic, H.H. Soleng, Supergravity domain walls, Phys. Rep. 282 (1997) 159-223, http://dx.doi.org/10.1016/S0370-1573(96)00035-X, arXiv:hepth/9604090.

[60] U. Gran, J. Gutowski, G. Papadopoulos, D. Roest, N=31 is not IIB, J. High Energy Phys. 02 (2007) 044, http://dx.doi.org/10.1088/1126-6708/2007/02/ 044, arXiv:hep-th/0606049.

[61] J.M. Figueroa-O'Farrill, S. Gadhia, M-theory preons cannot arise by quotients, J. High Energy Phys. 06 (2007) 043, http://dx.doi.org/10.1088/11266708/2007/06/043, arXiv:hep-th/0702055.

[62] J.M. Figueroa-O'Farrill, J. Gutowski, W. Sabra, The return of the four- and five-dimensional preons, Classical Quantum Gravity 24 (2007) 4429-4438, http://dx.doi.org/10.1088/0264-9381/24/17/010, arXiv:0705.2778.

[63] P.S. Howe, G. Papadopoulos, Twistor spaces for HKT manifolds, Phys. Lett. B 379 (1996) 80-86, http://dx.doi.org/10.1016/0370-2693(96)00393-0, arXiv:hep-th/9602108.

[64] J.C. Breckenridge, R.C. Myers, A.W. Peet, C. Vafa, D-branes and spinning black holes, Phys. Lett. B 391 (1997) 93-98, http://dx.doi.org/10.1016/S03702693(96)01460-8, arXiv:hep-th/9602065.

[65] G.W. Gibbons, S.W. Hawking, Gravitational multi - instantons, Phys. Lett. 78B (1978) 430, http://dx.doi.org/10.1016/0370-2693(78)90478-1.

[66] J.P. Gauntlett, J.B. Gutowski, Concentric black rings, Phys. Rev. D 71 (2005) 025013, http://dx.doi.org/10.1103/PhysRevD.71.025013, arXiv:hepth/0408010.

[67] J. Figueroa-O'Farrill, N. Hustler, The homogeneity theorem for supergravity backgrounds, J. High Energy Phys. 10 (2012) 014, http://dx.doi.org/10. 1007/JHEP10(2012)014, arXiv:1208.0553.

[68] J. Figueroa-O'Farrill, N. Hustler, Symmetric backgrounds of type IIB supergravity, Classical Quantum Gravity 30 (2013) 045008, http://dx.doi.org/10. 1088/0264-9381/30/4/045008, arXiv: 1209.4884

[69] J.P. Gauntlett, J.B. Gutowski, All supersymmetric solutions of minimal gauged supergravity in five-dimensions, Phys. Rev. D 68 (2003) 105009, http://dx.doi.org/10.1103/PhysRevD.68.105009, arXiv:hep-th/0304064; Phys. Rev. D 70 (2004) 089901, (erratum), http://dx.doi.org/10.1103/ PhysRevD.70.089901.

[70] J.B. Gutowski, H.S. Reall, Supersymmetric AdS(5) black holes, J. High Energy Phys. 02 (2004) 006, http://dx.doi.org/10.1088/1126-6708/2004/02/006, arXiv:hep-th/0401042.

[71] Z.W. Chong, M. Cvetic, H. Lu, C.N. Pope, General non-extremal rotating black holes in minimal five-dimensional gauged supergravity, Phys. Rev. Lett. 95 (2005) 161301, http://dx.doi.org/10.1103/PhysRevLett.95.161301, arXiv:hep-th/0506029.

[72] J.L. Blazquez-Salcedo, J. Kunz, F. Navarro-Lerida, E. Radu, Squashed, magnetized black holes in $D=5$ minimal gauged supergravity, J. High Energy Phys. 02 (2018) 061, http://dx.doi.org/10.1007/JHEP02(2018)061, arXiv:1711.10483.

[73] J. Grover, J.B. Gutowski, W. Sabra, Vanishing preons in the fifth dimension, Classical Quantum Gravity 24 (2007) 417-432, http://dx.doi.org/10.1088/ 0264-9381/24/2/009, arXiv:hep-th/0608187.

[74] H. Elvang, R. Emparan, D. Mateos, H.S. Reall, A supersymmetric black ring, Phys. Rev. Lett. 93 (2004) 211302, http://dx.doi.org/10.1103/PhysRevLett. 93.211302, arXiv:hep-th/0407065.

[75] G.T. Horowitz, H.K. Kunduri, J. Lucietti, Comments on black holes in bubbling spacetimes, J. High Energy Phys. 06 (2017) 048, http://dx.doi.org/10. 1007/JHEP06(2017)048, arXiv:1704.04071.

[76] H.K. Kunduri, J. Lucietti, Supersymmetric black holes with lens-space topology, Phys. Rev. Lett. 113 (21) (2014) 211101, http://dx.doi.org/10.1103/ PhysRevLett.113.211101, arXiv:1408.6083.

[77] S. Tomizawa, M. Nozawa, Supersymmetric black lenses in five dimensions, Phys. Rev. D 94 (4) (2016) 044037, http://dx.doi.org/10.1103/PhysRevD. 94.044037, arXiv:1606.06643.

[78] A.M. Ghezelbash, Supergravity solutions without tri-holomorphic U(1) isometries, Phys. Rev. D 78 (2008) 126002, http://dx.doi.org/10.1103/ PhysRevD.78.126002, arXiv:0811.2244.

[79] J.P. Gauntlett, J.B. Gutowski, General concentric black rings, Phys. Rev. D 71 (2005) 045002, http://dx.doi.org/10.1103/PhysRevD.71.045002, arXiv: hep-th/0408122.

[80] J.B. Gutowski, H.S. Reall, General supersymmetric AdS(5) black holes, J. High Energy Phys. 04 (2004) 048, http://dx.doi.org/10.1088/1126-6708/ 2004/04/048, arXiv:hep-th/0401129.

[81] J.B. Gutowski, W. Sabra, General supersymmetric solutions of five-dimensional supergravity, J. High Energy Phys. 10 (2005) 039, http://dx.doi.org/ 10.1088/1126-6708/2005/10/039, arXiv:hep-th/0505185.

[82] J.B. Gutowski, W.A. Sabra, Half-supersymmetric solutions in five-dimensional supergravity, J. High Energy Phys. 12 (2007) 025, http://dx.doi.org/10. 1088/1126-6708/2007/12/025, arXiv:0706.3147; J. High Energy Phys. 04 (2010) 042, (erratum), http://dx.doi.org/10.1007/JHEP04(2010)042.

[83] J. Grover, J.B. Gutowski, W. Sabra, Null half-supersymmetric solutions in five-dimensional supergravity, J. High Energy Phys. 10 (2008) 103, http://dx.doi.org/10.1088/1126-6708/2008/10/103, arXiv:0802.0231.

[84] R. Emparan, H.S. Reall, A rotating black ring solution in five-dimensions, Phys. Rev. Lett. 88 (2002) 101101, http://dx.doi.org/10.1103/PhysRevLett. 88.101101, arXiv:hep-th/0110260.

[85] I. Bena, N.P. Warner, One ring to rule them all ... and in the darkness bind them? Adv. Theor. Math. Phys. 9 (5) (2005) 667-701, http://dx.doi.org/10. 4310/ATMP.2005.v9.n5.a1, arXiv:hep-th/0408106.

[86] P. Berglund, E.G. Gimon, T.S. Levi, Supergravity microstates for BPS black holes and black rings, J. High Energy Phys. 06 (2006) 007, http://dx.doi.org/ 10.1088/1126-6708/2006/06/007, arXiv:hep-th/0505167.

[87] I. Bena, N.P. Warner, Bubbling supertubes and foaming black holes, Phys. Rev. D 74 (2006) 066001, http://dx.doi.org/10.1103/PhysRevD.74.066001, arXiv:hep-th/0505166. 
[88] I. Bena, C.-W. Wang, N.P. Warner, Mergers and typical black hole microstates, J. High Energy Phys. 11 (2006) 042, http://dx.doi.org/10.1088/11266708/2006/11/042, arXiv:hep-th/0608217.

[89] I. Bena, C.-W. Wang, N.P. Warner, Plumbing the Abyss: Black ring microstates, J. High Energy Phys. 07 (2008) 019, http://dx.doi.org/10.1088/11266708/2008/07/019, arXiv:0706.3786.

[90] I. Bena, C.-W. Wang, N.P. Warner, Black rings with varying charge density, J. High Energy Phys. 03 (2006) 015, http://dx.doi.org/10.1088/11266708/2006/03/015, arXiv:hep-th/0411072.

[91] G.T. Horowitz, H.S. Reall, How hairy can a black ring be? Classical Quantum Gravity 22 (2005) 1289-1302, http://dx.doi.org/10.1088/0264-9381/22/ 7/006, arXiv:hep-th/0411268.

[92] A. Tyukov, R. Walker, N.P. Warner, The structure of BPS equations for ambi-polar microstate geometries, 2018, arXiv:1807.06596.

[93] J. Bellorin, P. Meessen, T. Ortin, All the supersymmetric solutions of $\mathrm{N}=1, \mathrm{~d}=5$ ungauged supergravity, J. High Energy Phys. 01 (2007) 020, http: //dx.doi.org/10.1088/1126-6708/2007/01/020, arXiv:hep-th/0610196.

[94] J. Bellorin, Supersymmetric solutions of gauged five-dimensional supergravity with general matter couplings, Classical Quantum Gravity 26 (2009) 195012, http://dx.doi.org/10.1088/0264-9381/26/19/195012, arXiv:0810.0527.

[95] J.T. Liu, M. Mahato, D. Vaman, Mapping the G-structures and supersymmetric vacua of five-dimensional N=4 supergravity, Classical Quantum Gravity 24 (2007) 1115-1144, http://dx.doi.org/10.1088/0264-9381/24/5/006, arXiv:hep-th/0605268.

[96] A. Castro, J.L. Davis, P. Kraus, F. Larsen, String theory effects on five-dimensional black hole physics, Internat. J. Modern Phys. A 23 (2008) 613-691, http://dx.doi.org/10.1142/S0217751X08039724, arXiv:0801.1863.

[97] A. Castro, J.L. Davis, P. Kraus, F. Larsen, 5D black holes and strings with higher derivatives, J. High Energy Phys. 06 (2007) 007, http://dx.doi.org/10. 1088/1126-6708/2007/06/007, arXiv:hep-th/0703087.

[98] A. Castro, J.L. Davis, P. Kraus, F. Larsen, Precision entropy of spinning black holes, J. High Energy Phys. 09 (2007) 003, http://dx.doi.org/10.1088/11266708/2007/09/003, arXiv:0705.1847.

[99] F. Bonetti, D. Klemm, W.A. Sabra, P. Sloane, Spinorial geometry, off-shell Killing spinor identities and higher derivative 5D supergravities, J. High Energy Phys. 08 (2018) 121, http://dx.doi.org/10.1007/JHEP08(2018)121, arXiv:1806.04108.

[100] H. Nishino, S. Rajpoot, Alternative N=2 supergravity in five-dimensions with singularities, Phys. Lett. B 502 (2001) 246-258, http://dx.doi.org/10. 1016/S0370-2693(01)00146-0, arXiv:hep-th/0011066.

[101] T. Fujita, K. Ohashi, Superconformal tensor calculus in five-dimensions, Progr. Theoret. Phys. 106 (2001) 221-247, http://dx.doi.org/10.1143/PTP. 106.221, arXiv:hep-th/0104130.

[102] K. Hanaki, K. Ohashi, Y. Tachikawa, Supersymmetric completion of an $R^{2}$ term in five-dimensional supergravity, Progr. Theoret. Phys. 117 (2007) 533, http://dx.doi.org/10.1143/PTP.117.533, arXiv:hep-th/0611329.

[103] E.A. Bergshoeff, J. Rosseel, E. Sezgin, Off-shell D=5, N=2 Riemann squared supergravity, Classical Quantum Gravity 28 (2011) 225016, http://dx.doi. org/10.1088/0264-9381/28/22/225016, arXiv:1107.2825.

[104] P. Sloane, $\mathcal{N}=2$ dilaton-Weyl multiplets in 5D and Nishino-Rajpoot supergravity off-shell, J. High Energy Phys. 04 (2015) 062, http://dx.doi.org/ 10.1007/JHEP04(2015)062, arXiv:1409.6764.

[105] M.J. Duff, Strong / weak coupling duality from the dual string, Nuclear Phys. B 442 (1995) 47-63, http://dx.doi.org/10.1016/S0550-3213(95)00070-4, arXiv:hep-th/9501030.

[106] E. Witten, String theory dynamics in various dimensions, Nuclear Phys. B 443 (1995) 85-126, 333 (1995), http://dx.doi.org/10.1016/0550-3213(95) 00158-0, arXiv:hep-th/9503124.

[107] E. Witten, Some comments on string dynamics, in: Future perspectives in string theory. Proceedings, Conference, Strings'95, Los Angeles, USA, March 13-18, 1995, 1995, pp. 501-523, arXiv:hep-th/9507121.

[108] N. Marcus, J.H. Schwarz, Field theories that have no manifestly Lorentz invariant formulation, Phys. Lett. B 115 (1982) 111,111 (1982), http: //dx.doi.org/10.1016/0370-2693(82)90807-3.

[109] J.B. Gutowski, D. Martelli, H.S. Reall, All supersymmetric solutions of minimal supergravity in six- dimensions, Classical Quantum Gravity 20 (2003) 5049-5078, http://dx.doi.org/10.1088/0264-9381/20/23/008, arXiv:hep-th/0306235.

[110] A. Chamseddine, J.M. Figueroa-O'Farrill, W. Sabra, Supergravity vacua and Lorentzian Lie groups, 2003, arXiv:hep-th/0306278.

[111] H. Nishino, E. Sezgin, New couplings of six-dimensional supergravity, Nuclear Phys. B 505 (1997) 497-516, http://dx.doi.org/10.1016/S05503213(97)00357-X, arXiv:hep-th/9703075.

[112] S. Ferrara, F. Riccioni, A. Sagnotti, Tensor and vector multiplets in six-dimensional supergravity, Nuclear Phys. B 519 (1998) 115-140, http: //dx.doi.org/10.1016/S0550-3213(97)00837-7, arXiv:hep-th/9711059.

[113] F. Riccioni, All couplings of minimal six-dimensional supergravity, Nuclear Phys. B 605 (2001) 245-265, http://dx.doi.org/10.1016/S0550-3213(01) 00199-7, arXiv:hep-th/0101074.

[114] M. Akyol, G. Papadopoulos, Spinorial geometry and Killing spinor equations of 6-D supergravity, Classical Quantum Gravity 28 (2011) 105001, http://dx.doi.org/10.1088/0264-9381/28/10/105001, arXiv:1010.2632.

[115] U. Gran, P. Lohrmann, G. Papadopoulos, The spinorial geometry of supersymmetric heterotic string backgrounds, J. High Energy Phys. 02 (2006) 063 , http://dx.doi.org/10.1088/1126-6708/2006/02/063, arXiv:hep-th/0510176.

[116] P.S. Howe, G. Papadopoulos, Twistor spaces for HKT manifolds, Phys. Lett. B379 (1996) 80-86, http://dx.doi.org/10.1016/0370-2693(96)00393-0, arXiv:hep-th/9602108.

[117] P.S. Howe, A. Opfermann, G. Papadopoulos, Twistor spaces for QKT manifolds, Comm. Math. Phys. 197 (1998) 713-727, http://dx.doi.org/10.1007/ s002200050469, arXiv:hep-th/9710072.

[118] G. Papadopoulos, Rotating rotated branes, J. High Energy Phys. 04 (1999) 014, http://dx.doi.org/10.1088/1126-6708/1999/04/014, arXiv:hepth/9902166.

[119] J. Ford, S. Giusto, A. Saxena, A class of BPS time-dependent 3-charge microstates from spectral flow, Nuclear Phys. B 790 (2008) 258-280, http: //dx.doi.org/10.1016/j.nuclphysb.2007.09.008, arXiv:hep-th/0612227.

[120] I. Bena, S. Giusto, M. Shigemori, N.P. Warner, Supersymmetric solutions in six dimensions: A linear structure, J. High Energy Phys. 03 (2012) 084 , http://dx.doi.org/10.1007/JHEP03(2012)084, arXiv:1110.2781.

[121] H. Het Lam, S. Vandoren, BPS solutions of six-dimensional $(1,0)$ supergravity coupled to tensor multiplets, J. High Energy Phys. 06 (2018) 021 , http://dx.doi.org/10.1007/JHEP06(2018)021, arXiv:1804.04681.

[122] M. Cariglia, O.A.P. Mac Conamhna, The general form of supersymmetric solutions of $\mathrm{N}=(1,0) \mathrm{U}(1)$ and $\mathrm{SU}(2)$ gauged supergravities in six-dimensions, Classical Quantum Gravity 21 (2004) 3171-3196, http://dx.doi.org/10.1088/0264-9381/21/13/006, arXiv:hep-th/0402055.

[123] P.A. Cano, T. Ortin, All the supersymmetric solutions of ungauged $\mathcal{N}=(1,0), d=6$ supergravity, 2018, arXiv:1804.04945.

[124] D.J. Gross, J.A. Harvey, E.J. Martinec, R. Rohm, Heterotic string theory. 1. The free heterotic string, Nuclear Phys. B 256 (1985) 253, http://dx.doi.org/ 10.1016/0550-3213(85)90394-3.

[125] D.J. Gross, J.A. Harvey, E.J. Martinec, R. Rohm, Heterotic string theory. 2. The interacting heterotic string, Nuclear Phys. B 267 (1986) 75-124, http://dx.doi.org/10.1016/0550-3213(86)90146-X.

[126] U. Gran, G. Papadopoulos, D. Roest, P. Sloane, Geometry of all supersymmetric type I backgrounds, J. High Energy Phys. 08 (2007) 074, http: //dx.doi.org/10.1088/1126-6708/2007/08/074, arXiv:hep-th/0703143. 
[127] U. Gran, G. Papadopoulos, D. Roest, Supersymmetric heterotic string backgrounds, Phys. Lett. B 656 (2007) 119-126, http://dx.doi.org/10.1016/j. physletb.2007.09.024, arXiv:0706.4407.

[128] U. Gran, P. Lohrmann, G. Papadopoulos, Geometry of type II common sector N=2 backgrounds, J. High Energy Phys. 06 (2006) 049, http://dx.doi.org/ 10.1088/1126-6708/2006/06/049, arXiv:hep-th/0602250.

[129] M.B. Green, J.H. Schwarz, Anomaly cancellation in supersymmetric D=10 gauge theory and superstring theory, Phys. Lett. 149B (1984) 117-122, http://dx.doi.org/10.1016/0370-2693(84)91565-X.

[130] C.M. Hull, P.K. Townsend, The two loop beta function for $\sigma$ models with torsion, Phys. Lett. B 191 (1987) 115-121, http://dx.doi.org/10.1016/03702693(87)91331-1.

[131] A. Strominger, Superstrings with Torsion, Nuclear Phys. B 274 (1986) 253, http://dx.doi.org/10.1016/0550-3213(86)90286-5.

[132] C.M. Hull, Compactifications of the heterotic superstring, Phys. Lett. B 178 (1986) 357-364, http://dx.doi.org/10.1016/0370-2693(86)91393-6.

[133] E.A. Bergshoeff, M. de Roo, The quartic effective action of the heterotic string and supersymmetry, Nuclear Phys. B 328 (1989) 439-468, http: //dx.doi.org/10.1016/0550-3213(89)90336-2.

[134] B. de Wit, D.J. Smit, N.D. Hari Dass, Residual supersymmetry of compactified D=10 supergravity, Nuclear Phys. B 283 (1987) 165, http://dx.doi.org/ 10.1016/0550-3213(87)90267-7.

[135] U. Gran, G. Papadopoulos, D. Roest, Supersymmetric heterotic string backgrounds, Phys. Lett. B 656 (2007) 119-126, http://dx.doi.org/10.1016/j. physletb.2007.09.024, arXiv:0706.4407.

[136] G. Papadopoulos, Heterotic supersymmetric backgrounds with compact holonomy revisited, Classical Quantum Gravity 27 (2010) 125008, http: //dx.doi.org/10.1088/0264-9381/27/12/125008, arXiv:0909.2870.

[137] S. Ivanov, Connection with torsion, parallel spinors and geometry of spin(7) manifolds, 2001, arXiv:math/0111216.

[138] S. Ivanov, G. Papadopoulos, A no go theorem for string warped compactifications, Phys. Lett. B 497 (2001)309-316, http://dx.doi.org/10.1016/S03702693(00)01330-7, arXiv:hep-th/0008232.

[139] G. Papadopoulos, A.A. Tseytlin, Complex geometry of conifolds and five-brane wrapped on two sphere, Classical Quantum Gravity 18 (2001) $1333-$ 1354, http://dx.doi.org/10.1088/0264-9381/18/7/315, arXiv:hep-th/0012034.

[140] G. Lopes Cardoso, G. Curio, G. Dall'Agata, D. Lust, P. Manousselis, G. Zoupanos, NonKahler string backgrounds and their five torsion classes, Nuclear Phys. B 652 (2003) 5-34, http://dx.doi.org/10.1016/S0550-3213(03)00049-X, arXiv:hep-th/0211118.

[141] S. Chiossi, S. Salamon, The Intrinsic torsion of SU(3) and G(2) structures, J. Diff. Geom. (2002) 115-133, http://dx.doi.org/10.1142/9789812777751 0010, arXiv:math/0202282.

[142] J.P. Gauntlett, D. Martelli, S. Pakis, D. Waldram, G structures and wrapped NS5-branes, Comm. Math. Phys. 247 (2004) 421-445, http://dx.doi.org/ 10.1007/s00220-004-1066-y, arXiv:hep-th/0205050.

[143] J.P. Gauntlett, D. Martelli, D. Waldram, Superstrings with intrinsic torsion, Phys. Rev. D 69 (2004) 086002, http://dx.doi.org/10.1103/PhysRevD.69 086002, arXiv:hep-th/0302158.

[144] P. Nurowski, A. Trautman, Robinson manifolds as the Lorentzian analoges of Hermite manifolds, 2002, arXiv:math/0201266.

[145] G. Papadopoulos, New half supersymmetric solutions of the heterotic string, Classical Quantum Gravity 26 (2009) 135001, http://dx.doi.org/10. 1088/0264-9381/26/13/135001, arXiv:0809.1156.

[146] J.M. Figueroa-O'Farrill, E. Hackett-Jones, G. Moutsopoulos, The Killing superalgebra of ten-dimensional supergravity backgrounds, Classical Quantum Gravity 24 (2007) 3291-3308, http://dx.doi.org/10.1088/0264-9381/24/13/010, arXiv:hep-th/0703192.

[147] T. Friedrich, S. Ivanov, Parallel spinors and connections with skew symmetric torsion in string theory, Asian J. Math. 6 (2002) 303-336, arXiv: math/0102142.

[148] T. Friedrich, S. Ivanov, Killing spinor equations in dimension 7 and geometry of integrable G(2) manifolds, J. Geom. Phys. 48 (2003) 1, http: //dx.doi.org/10.1016/S0393-0440(03)00005-6, arXiv:math/0112201.

[149] A. Sen, (2, 0) supersymmetry and space-time supersymmetry in the heterotic string theory, Nuclear Phys. B 278 (1986) 289-308, http://dx.doi.org/ $10.1016 / 0550-3213(86) 90214-2$

[150] P.S. Howe, G. Papadopoulos, Anomalies in two-dimensional supersymmetric nonlinear $\sigma$ models, Classical Quantum Gravity 4 (1987) 1749-1766, http://dx.doi.org/10.1088/0264-9381/4/6/027.

[151] A. Opfermann, G. Papadopoulos, Homogeneous HKT and QKT manifolds, 1998, arXiv:math-ph/9807026.

[152] J. Li, S.-T. Yau, The existence of supersymmetric string theory with torsion, J. Differential Geom. 70 (1) (2005) 143-181, arXiv:hep-th/0411136.

[153] M. Fernandez, S. Ivanov, L. Ugarte, D. Vassilev, Quaternionic Heisenberg group and heterotic string solutions with non-constant dilaton in dimensions 7 and 5, Comm. Math. Phys. 339 (1)(2015) 199-219, http://dx.doi.org/10.1007/s00220-015-2397-6, arXiv:1410.4130.

[154] T. Kawano, S. Yamaguchi, Dilatonic parallelizable NS NS backgrounds, Phys. Lett. B 568 (2003) 78-82, http://dx.doi.org/10.1016/j.physletb.2003.06. 022, arXiv:hep-th/0306038.

[155] J.M. Figueroa-O'Farrill, T. Kawano, S. Yamaguchi, Parallelizable heterotic backgrounds, J. High Energy Phys. 10 (2003) 012, http://dx.doi.org/10.1088/ 1126-6708/2003/10/012, arXiv:hep-th/0308141.

[156] C.G. Callan Jr., J.A. Harvey, A. Strominger, World sheet approach to heterotic instantons and solitons, Nuclear Phys. B 359 (1991) 611-634, http: //dx.doi.org/10.1016/0550-3213(91)90074-8.

[157] A. Dabholkar, G.W. Gibbons, J.A. Harvey, F. Ruiz Ruiz, Superstrings and solitons, Nuclear Phys. B 340 (1990) 33-55, http://dx.doi.org/10.1016/05503213(90)90157-9.

[158] G.W. Gibbons, Aspects of supergravity theories, in: XV GIFT Seminar on Supersymmetry and Supergravity Gerona, Spain, June 4-11, 1984, 1984.

[159] J.M. Maldacena, C. Nunez, Supergravity description of field theories on curved manifolds and a no go theorem, in: Superstrings. Proceedings, International Conference, Strings 2000, Ann Arbor, USA, July 10-15, 2000, Internat. J. Modern Phys. A 16 (2001) 822-855, 182 (2000), http: //dx.doi.org/10.1142/S0217751X01003937, arXiv:hep-th/0007018.

[160] S. Ivanov, G. Papadopoulos, Vanishing theorems and string backgrounds, Classical Quantum Gravity 18 (2001) 1089-1110, http://dx.doi.org/10. 1088/0264-9381/18/6/309, arXiv:math/0010038.

[161] M. Gunaydin, H. Nicolai, Seven-dimensional octonionic Yang-Mills instanton and its extension to an heterotic string soliton, Phys. Lett. B 351 (1995) 169-172, http://dx.doi.org/10.1016/0370-2693(95)00375-U, arXiv:hep-th/9502009; Phys. Lett. B 376 (1996) 329, (addendum)

[162] G. Papadopoulos, P.K. Townsend, Compactification of D = 11 supergravity on spaces of exceptional holonomy, Phys. Lett. B 357 (1995) $300-306$ http://dx.doi.org/10.1016/0370-2693(95)00929-F, arXiv:hep-th/9506150.

[163] P. Candelas, G.T. Horowitz, A. Strominger, E. Witten, Vacuum configurations for superstrings, Nuclear Phys. B 258 (1985) 46-74, http://dx.doi.org/ 10.1016/0550-3213(85)90602-9.

[164] P. Candelas, M. Lynker, R. Schimmrigk, Calabi-Yau manifolds in weighted P(4), Nuclear Phys. B 341 (1990) 383-402, http://dx.doi.org/10.1016/05503213(90)90185-G.

[165] B.R. Greene, M.R. Plesser, Duality in Calabi-Yau moduli space, Nuclear Phys. B 338 (1990) 15-37, http://dx.doi.org/10.1016/0550-3213(90)90622-K.

[166] P.S. Aspinwall, C.A. Lutken, G.G. Ross, Construction and couplings of mirror manifolds, Phys. Lett. B 241 (1990) 373-380, http://dx.doi.org/10.1016/ 0370-2693(90)91659-Y.

[167] A.A. Tseytlin, Extreme dyonic black holes in string theory, Modern Phys. Lett. A 11 (1996) 689-714, http://dx.doi.org/10.1142/S0217732396000709, arXiv:hep-th/9601177. 
[168] C.G. Callan, J.M. Maldacena, D-brane approach to black hole quantum mechanics, Nuclear Phys. B 472 (1996) 591-610, http://dx.doi.org/10.1016/ 0550-3213(96)00225-8, arXiv:hep-th/9602043.

[169] A. Strominger, C. Vafa, Microscopic origin of the Bekenstein-Hawking entropy, Phys. Lett. B 379 (1996) 99-104, http://dx.doi.org/10.1016/03702693(96)00345-0, arXiv:hep-th/9601029.

[170] P.A. Cano, P. Meessen, T. Ortin, P.F. Ramirez, $\alpha^{\prime}$-corrected black holes in string theory, J. High Energy Phys. 05 (2018) 110, http://dx.doi.org/10.1007/ JHEP05(2018)110, arXiv: 1803.01919.

[171] G. Papadopoulos, A. Teschendorff, Grassmannians, calibrations and five-brane intersections, Classical Quantum Gravity 17 (2000) 2641-2662, http://dx.doi.org/10.1088/0264-9381/17/14/304, arXiv:hep-th/9811034.

[172] A.H. Chamseddine, M.S. Volkov, NonAbelian BPS monopoles in N=4 gauged supergravity, Phys. Rev. Lett. 79 (1997) 3343-3346, http://dx.doi.org/10. 1103/PhysRevLett.79.3343, arXiv:hep-th/9707176.

[173] J.M. Maldacena, C. Nunez, Towards the large N limit of pure N=1 superYang-Mills, Phys. Rev. Lett. 86 (2001) 588-591, http://dx.doi.org/10.1103/ PhysRevLett.86.588, arXiv:hep-th/0008001.

[174] E. Cremmer, B. Julia, J. Scherk, Supergravity theory in eleven-dimensions, Phys. Lett. B 76 (1978) 409-412, 25 (1978), http://dx.doi.org/10.1016/03702693(78)90894-8.

[175] M.J. Duff, K.S. Stelle, Multimembrane solutions of D = 11 supergravity, Phys. Lett. B 253 (1991) 113-118, 110 (1990), http://dx.doi.org/10.1016/03702693(91)91371-2.

[176] R. Gueven, Black p-brane solutions of D = 11 supergravity theory, Phys. Lett. B 276 (1992) 49-55, 135 (1992), http://dx.doi.org/10.1016/03702693(92)90540-K.

[177] G. Papadopoulos, P.K. Townsend, Intersecting M-branes, Phys. Lett. B 380 (1996) 273-279, 279 (1996), http://dx.doi.org/10.1016/0370-2693(96) 00506-0, arXiv:hep-th/9603087.

[178] A.A. Tseytlin, Harmonic superpositions of M-branes, Nuclear Phys. B 475 (1996) 149-163, 286 (1996), http://dx.doi.org/10.1016/0550-3213(96) 00328-8, arXiv:hep-th/9604035.

[179] J.P. Gauntlett, D.A. Kastor, J.H. Traschen, Overlapping branes in M theory, Nuclear Phys. B 478 (1996) 544-560, http://dx.doi.org/10.1016/05503213(96)00423-3, arXiv:hep-th/9604179.

[180] M.J. Duff, R.R. Khuri, J.X. Lu, String solitons, Phys. Rep. 259 (1995) 213-326, http://dx.doi.org/10.1016/0370-1573(95)00002-X, arXiv:hep-th/ 9412184.

[181] R. Bryant, Pseudo-Riemannian metrics with parallel spinor fields and vanishing Ricci tensor, Semin. Congr., Soc. Math. France, Paris 4 (2000) 53-94, arXiv:math/0004073.

[182] J.M. Figueroa-O'Farrill, Breaking the M waves, Classical Quantum Gravity 17 (2000) 2925-2948, http://dx.doi.org/10.1088/0264-9381/17/15/306, arXiv:hep-th/9904124.

[183] J.P. Gauntlett, S. Pakis, The geometry of D = 11 killing spinors, J. High Energy Phys. 04 (2003) 039, http://dx.doi.org/10.1088/1126-6708/2003/04/039, arXiv:hep-th/0212008.

[184] J.P. Gauntlett, J.B. Gutowski, S. Pakis, The geometry of D = 11 null Killing spinors, J. High Energy Phys. 12 (2003) 049, http://dx.doi.org/10.1088/11266708/2003/12/049, arXiv:hep-th/0311112.

[185] U. Gran, G. Papadopoulos, D. Roest, Systematics of M-theory spinorial geometry, Classical Quantum Gravity 22 (2005) 2701-2744, http://dx.doi.org/ 10.1088/0264-9381/22/13/013, arXiv:hep-th/0503046.

[186] U. Gran, J. Gutowski, G. Papadopoulos, The spinorial geometry of supersymmetric IIB backgrounds, Classical Quantum Gravity 22 (2005) 2453-2492, http://dx.doi.org/10.1088/0264-9381/22/12/010, arXiv:hep-th/0501177.

[187] U. Gran, J. Gutowski, G. Papadopoulos, The G(2) spinorial geometry of supersymmetric IIB backgrounds, Classical Quantum Gravity 23 (2006) $143-$ 206, http://dx.doi.org/10.1088/0264-9381/23/1/009, arXiv:hep-th/0505074.

[188] U. Gran, J. Gutowski, G. Papadopoulos, D. Roest, Systematics of IIB spinorial geometry, Classical Quantum Gravity 23 (2006) 1617-1678, http: //dx.doi.org/10.1088/0264-9381/23/5/012, arXiv:hep-th/0507087.

[189] U. Gran, G. Papadopoulos, C. von Schultz, Supersymmetric geometries of IIA supergravity I, J. High Energy Phys. 05 (2014) 024, http://dx.doi.org/10. 1007/JHEP05(2014)024, arXiv:1401.6900.

[190] U. Gran, G. Papadopoulos, C. von Schultz, Supersymmetric geometries of IIA supergravity II, J. High Energy Phys. 12 (2015) 113, http://dx.doi.org/10. 1007/JHEP12(2015)113, arXiv:1508.05006.

[191] U. Gran, G. Papadopoulos, C. von Schultz, Supersymmetric geometries of IIA supergravity III, J. High Energy Phys. 06 (2016) 045, http://dx.doi.org/ 10.1007/JHEP06(2016)045, arXiv:1602.07934.

[192] J.H. Schwarz, P.C. West, Symmetries and transformations of chiral N=2 D=10 supergravity, Phys. Lett. 126B (1983) 301-304, http://dx.doi.org/10. 1016/0370-2693(83)90168-5.

[193] J.H. Schwarz, Covariant field equations of chiral N=2 D=10 supergravity, Nuclear Phys. B 226 (1983) 269, 269 (1983), http://dx.doi.org/10.1016/05503213(83)90192-X.

[194] M. Huq, M.A. Namazie, Kaluza-Klein supergravity in ten-dimensions, Classical Quantum Gravity 2 (1985) 293, 293 (1983), http://dx.doi.org/10.1088/ 0264-9381/2/3/007.

[195] F. Giani, M. Pernici, N=2 supergravity in ten-dimensions, Phys. Rev. D 30 (1984) 325-333, http://dx.doi.org/10.1103/PhysRevD.30.325.

[196] I.C.G. Campbell, P.C. West, N=2 D=10 nonchiral supergravity and its spontaneous compactification, Nuclear Phys. B 243 (1984) 112-124, http: //dx.doi.org/10.1016/0550-3213(84)90388-2.

[197] L.J. Romans, Massive N=2a supergravity in ten-dimensions, Phys. Lett. B 169 (1986) 374, 374 (1985), http://dx.doi.org/10.1016/0370-2693(86) 90375-8.

[198] V.G. Kac, A sketch of Lie superalgebra theory, Comm. Math. Phys. 53 (1977) 31-64, http://dx.doi.org/10.1007/BF01609166.

[199] J.P. Gauntlett, R.C. Myers, P.K. Townsend, Supersymmetry of rotating branes, Phys. Rev. D 59 (1998) 025001, http://dx.doi.org/10.1103/PhysRevD. 59.025001, arXiv:hep-th/9809065.

[200] J.M. Figueroa-O'Farrill, On the supersymmetries of Anti-de Sitter vacua, Classical Quantum Gravity 16 (1999) 2043-2055, http://dx.doi.org/10.1088/ 0264-9381/16/6/330, arXiv:hep-th/9902066.

[201] J.M. Figueroa-O'Farrill, G. Papadopoulos, Plucker type relations for orthogonal planes, J. Geom. Phys. 49 (2004) 294, http://dx.doi.org/10.1016/S03930440(03)00093-7, arXiv:math/0211170.

[202] P.G.O. Freund, M.A. Rubin, Dynamics of dimensional reduction, Phys. Lett. B 97 (1980) 233-235, 80 (1980), http://dx.doi.org/10.1016/0370-2693(80) 90590-0.

[203] M.J. Duff, C.N. Pope, Kaluza-Klein supergravity and the seven sphere, in: September School on Supergravity and Supersymmetry Trieste, Italy, September 6-18, 1982, 1983, pp. 183-228.

[204] K. Pilch, P. van Nieuwenhuizen, P.K. Townsend, Compactification of $d=11$ supergravity on S(4) (Or $11=7+4$, too), Nuclear Phys. B $242(1984)$ 377-392, http://dx.doi.org/10.1016/0550-3213(84)90400-0.

[205] J. Kowalski-Glikman, Vacuum states in supersymmetric Kaluza-Klein theory, Phys. Lett. 134B (1984) 194-196, http://dx.doi.org/10.1016/03702693(84)90669-5. 
[206] M. Blau, J.M. Figueroa-O'Farrill, C. Hull, G. Papadopoulos, Penrose limits and maximal supersymmetry, Classical Quantum Gravity 19 (2002) L87-L95, http://dx.doi.org/10.1088/0264-9381/19/10/101, arXiv:hep-th/0201081.

[207] M. Blau, J.M. Figueroa-O'Farrill, C. Hull, G. Papadopoulos, A new maximally supersymmetric background of IIB superstring theory, J. High Energy Phys. 01 (2002) 047, http://dx.doi.org/10.1088/1126-6708/2002/01/047, arXiv:hep-th/0110242.

[208] V. Filippov, n-Lie algebras, Sibirsk. Mat. Zh. 26 (1985) 126-140.

[209] U. Gran, J. Gutowski, G. Papadopoulos, D. Roest, N=31, D=11, J. High Energy Phys. 02 (2007) 043, http://dx.doi.org/10.1088/1126-6708/2007/02/043, arXiv:hep-th/0610331.

[210] I.A. Bandos, J.A. de Azcarraga, O. Varela, On the absence of BPS preonic solutions in IIA and IIB supergravities, J. High Energy Phys. 09 (2006) 009 , http://dx.doi.org/10.1088/1126-6708/2006/09/009, arXiv:hep-th/0607060.

[211] U. Gran, J. Gutowski, G. Papadopoulos, D. Roest, IIB solutions with N > 28 Killing spinors are maximally supersymmetric, J. High Energy Phys. 12 (2007) 070, http://dx.doi.org/10.1088/1126-6708/2007/12/070, arXiv:0710.1829.

[212] U. Gran, J. Gutowski, G. Papadopoulos, Classification of IIB backgrounds with 28 supersymmetries, J. High Energy Phys. 01 (2010) 044, http: //dx.doi.org/10.1007/JHEP01(2010)044, arXiv:0902.3642.

[213] I. Bena, R. Roiban, Supergravity pp wave solutions with twenty eight supercharges and twenty four supercharges, Phys. Rev. D 67 (2003) 125014 http://dx.doi.org/10.1103/PhysRevD.67.125014, arXiv:hep-th/0206195.

[214] U. Gran, J. Gutowski, G. Papadopoulos, M-theory backgrounds with 30 Killing spinors are maximally supersymmetric, J. High Energy Phys. 03 (2010) 112, http://dx.doi.org/10.1007/JHEP03(2010)112, arXiv:1001.1103.

[215] B. Carter, Black holes equilibrium states, in: Proceedings, Ecole d'Eté de Physique Théorique: Les Astres Occlus: Les Houches, France, August, 1972, 1973, pp. 57-214.

[216] G.W. Gibbons, G.T. Horowitz, P.K. Townsend, Higher dimensional resolution of dilatonic black hole singularities, Classical Quantum Gravity 12 (1995) 297-318, http://dx.doi.org/10.1088/0264-9381/12/2/004, arXiv:hep-th/9410073.

[217] A. Sen, Black hole entropy function, attractors and precision counting of microstates, Gen. Relativity Gravitation 40 (2008) 2249-2431, http: //dx.doi.org/10.1007/s10714-008-0626-4, arXiv:0708.1270.

[218] S.W. Hawking, Black holes in general relativity, Comm. Math. Phys. 25 (1972) 152-166, http://dx.doi.org/10.1007/BF01877517.

[219] V. Moncrief, J. Isenberg, Symmetries of cosmological Cauchy horizons, Comm. Math. Phys. 89 (3) (1983) 387-413, http://dx.doi.org/10.1007/ BF01214662.

[220] H. Friedrich, I. Racz, R.M. Wald, On the rigidity theorem for space-times with a stationary event horizon or a compact Cauchy horizon, Comm. Math. Phys. 204 (1999) 691-707, http://dx.doi.org/10.1007/s002200050662, arXiv:gr-qc/9811021.

[221] J. Gutowski, G. Papadopoulos, Index theory and dynamical symmetry enhancement of M-horizons, J. High Energy Phys. 05 (2013) 088, http: //dx.doi.org/10.1007/JHEP05(2013)088, arXiv:1303.0869.

[222] U. Gran, J. Gutowski, G. Papadopoulos, Index theory and dynamical symmetry enhancement near IIB horizons, J. High Energy Phys. 11 (2013) 104, http://dx.doi.org/10.1007/JHEP11(2013)104, arXiv:1306.5765.

[223] U. Gran, J. Gutowski, U. Kayani, G. Papadopoulos, Dynamical symmetry enhancement near IIA horizons, J. High Energy Phys. 06 (2015) 139, http://dx.doi.org/10.1007/JHEP06(2015)139, arXiv:1409.6303.

[224] U. Gran, J. Gutowski, U. Kayani, G. Papadopoulos, Dynamical symmetry enhancement near massive IIA horizons, Classical Quantum Gravity 32 (23) (2015) 235004, http://dx.doi.org/10.1088/0264-9381/32/23/235004, arXiv:1411.5286.

[225] A. Fontanella, J.B. Gutowski, G. Papadopoulos, Anomaly corrected heterotic horizons, J. High Energy Phys. 10 (2016) 121, http://dx.doi.org/10.1007/ JHEP10(2016)121, arXiv:1605.05635.

[226] J. Grover, J.B. Gutowski, G. Papadopoulos, W.A. Sabra, Index theory and supersymmetry of 5D horizons, J. High Energy Phys. 06 (2014) 020, http://dx.doi.org/10.1007/JHEP06(2014)020, arXiv:1303.0853.

[227] J. Gutowski, T. Mohaupt, G. Papadopoulos, Dynamical symmetry enhancement near $\mathcal{N}=2$, D = 4 gauged supergravity horizons, J. High Energy Phys 03 (2017) 150, http://dx.doi.org/10.1007/JHEP03(2017)150, arXiv:1607.02877.

[228] U. Kayani, Symmetry enhancement of extremal horizons in D=5 supergravity, Classical Quantum Gravity 35 (12) (2018) 125013, http://dx.doi.org/ 10.1088/1361-6382/aac30c, arXiv: 1801.08833.

[229] M.J. Duff, B.E.W. Nilsson, C.N. Pope, Kaluza-Klein supergravity, Phys. Rep. 130 (1986) 1-142, http://dx.doi.org/10.1016/0370-1573(86)90163-8.

[230] M. Grana, Flux compactifications in string theory: A comprehensive review, Phys. Rep. 423 (2006) 91-158, http://dx.doi.org/10.1016/j.physrep.2005 10.008, arXiv:hep-th/0509003.

[231] O. Aharony, S.S. Gubser, J.M. Maldacena, H. Ooguri, Y. Oz, Large N field theories, string theory and gravity, Phys. Rep. 323 (2000) 183-386, http://dx.doi.org/10.1016/S0370-1573(99)00083-6, arXiv:hep-th/9905111.

[232] U. Gran, J. Gutowski, G. Papadopoulos, AdS backgrounds from black hole horizons, Classical Quantum Gravity 30 (2013) 055014, http://dx.doi.org/ 10.1088/0264-9381/30/5/055014, arXiv: 1110.0479.

[233] L. Castellani, L.J. Romans, N.P. Warner, A classification of compactifying solutions for $d=11$ supergravity, Nuclear Phys. B 241 (1984) 429-462, http://dx.doi.org/10.1016/0550-3213(84)90055-5.

[234] I.R. Klebanov, E. Witten, Superconformal field theory on three-branes at a Calabi-Yau singularity, Nuclear Phys. B 536 (1998) 199-218, http: //dx.doi.org/10.1016/S0550-3213(98)00654-3, arXiv:hep-th/9807080.

[235] B.S. Acharya, J.M. Figueroa-O'Farrill, C.M. Hull, B.J. Spence, Branes at conical singularities and holography, Adv. Theor. Math. Phys. 2 (1999) 12491286, http://dx.doi.org/10.4310/ATMP.1998.v2.n6.a2, arXiv:hep-th/9808014.

[236] M. Cvetic, H. Lu, C.N. Pope, J.F. Vazquez-Poritz, AdS in warped space-times, Phys. Rev. D 62 (2000) 122003, http://dx.doi.org/10.1103/PhysRevD.62 122003, arXiv:hep-th/0005246.

[237] J.P. Gauntlett, D. Martelli, J. Sparks, D. Waldram, Supersymmetric AdS(5) solutions of M theory, Classical Quantum Gravity 21 (2004) 4335-4366, http://dx.doi.org/10.1088/0264-9381/21/18/005, arXiv:hep-th/0402153.

[238] J.P. Gauntlett, D. Martelli, J. Sparks, D. Waldram, Supersymmetric AdS(5) solutions of type IIB supergravity, Classical Quantum Gravity 23 (2006) 4693-4718, http://dx.doi.org/10.1088/0264-9381/23/14/009, arXiv:hep-th/0510125.

[239] J.P. Gauntlett, O.A.P. Mac Conamhna, T. Mateos, D. Waldram, AdS spacetimes from wrapped M5 branes, J. High Energy Phys. 11 (2006) 053, http://dx.doi.org/10.1088/1126-6708/2006/11/053, arXiv:hep-th/0605146.

[240] D. Gaiotto, J. Maldacena, The gravity duals of N=2 superconformal field theories, J. High Energy Phys. 10 (2012) 189, http://dx.doi.org/10.1007/ JHEP10(2012)189, arXiv:0904.4466.

[241] D. Lust, D. Tsimpis, New supersymmetric AdS(4) type II vacua, J. High Energy Phys. 09(2009) 098, http://dx.doi.org/10.1088/1126-6708/2009/09/098, arXiv:0906.2561.

[242] K. Pilch, N.P. Warner, A New supersymmetric compactification of chiral IIB supergravity, Phys. Lett. B 487 (2000) 22-29, http://dx.doi.org/10.1016/ S0370-2693(00)00796-6, arXiv:hep-th/0002192.

[243] N. Kim, J.-D. Park, Comments on AdS(2) solutions of D=11 supergravity, J. High Energy Phys. 09 (2006) 041, http://dx.doi.org/10.1088/11266708/2006/09/041, arXiv:hep-th/0607093.

[244] G. Itsios, C. Nunez, K. Sfetsos, D.C. Thompson, On non-abelian t-duality and new N=1 backgrounds, Phys. Lett. B721 (2013) 342-346, http://dx.doi. org/10.1016/j.physletb.2013.03.033, arXiv:1212.4840. 


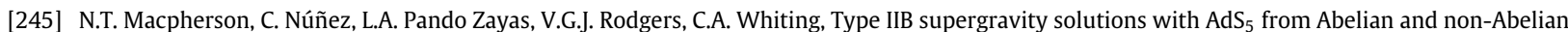
T dualities, J. High Energy Phys. 02 (2015) 040, http://dx.doi.org/10.1007/JHEP02(2015)040, arXiv:1410.2650.

[246] E. D’Hoker, M. Gutperle, C.F. Uhlemann, Holographic duals for five-dimensional superconformal quantum field theories, Phys. Rev. Lett. 118 (10) (2017) 101601, http://dx.doi.org/10.1103/PhysRevLett.118.101601, arXiv:1611.09411.

[247] F. Apruzzi, M. Fazzi, A. Passias, A. Rota, A. Tomasiello, Six-dimensional superconformal theories and their compactifications from type IIA supergravity, Phys. Rev. Lett. 115 (6) (2015) 061601, http://dx.doi.org/10.1103/PhysRevLett.115.061601, arXiv:1502.06616.

[248] F. Apruzzi, M. Fazzi, A. Passias, D. Rosa, A. Tomasiello, AdS 6 solutions of type II supergravity, J. High Energy Phys. 11 (2014) 099, http://dx.doi.org/ 10.1007/JHEP11(2014)099, arXiv:1406.0852; JHEP 05 (2015) 012, (erratum), http://dx.doi.org/10.1007/JHEP05(2015)012.

[249] S.W. Beck, J.B. Gutowski, G. Papadopoulos, Geometry and supersymmetry of heterotic warped flux AdS backgrounds, J. High Energy Phys. 07 (2015) 152, http://dx.doi.org/10.1007/JHEP07(2015)152, arXiv:1505.01693.

[250] J.B. Gutowski, G. Papadopoulos, Supersymmetry of AdS and flat backgrounds in M-theory, J. High Energy Phys. 02 (2015) 145, http://dx.doi.org/10. 1007/JHEP02(2015)145, arXiv:1407.5652.

[251] S.W. Beck, J.B. Gutowski, G. Papadopoulos, Supersymmetry of AdS and flat IIB backgrounds, J. High Energy Phys. 02 (2015) 020, http://dx.doi.org/10. 1007/JHEP02(2015)020, arXiv:1410.3431.

[252] S. Beck, J.B. Gutowski, G. Papadopoulos, Supersymmetry of IIA warped flux AdS and flat backgrounds, J. High Energy Phys. 09 (2015) 135, http: //dx.doi.org/10.1007/JHEP09(2015)135, arXiv:1501.07620.

[253] U. Gran, J.B. Gutowski, G. Papadopoulos, On supersymmetric Anti-de-Sitter, de-Sitter and Minkowski flux backgrounds, Classical Quantum Gravity 35 (6) (2018) 065016, http://dx.doi.org/10.1088/1361-6382/aaac8c, arXiv:1607.00191.

[254] U.H. Danielsson, T. Van Riet, What if string theory has no de Sitter vacua? 2018, arXiv: 1804.01120.

[255] S. Beck, U. Gran, J. Gutowski, G. Papadopoulos, All killing superalgebras for warped AdS backgrounds, JHEP 12 (2018) 047, http://dx.doi.org/10.1007/ JHEP12(2018)047, arXiv:hep-th/1710.03713.

[256] R. D'Auria, P. Fre, Spontaneous generation of osp(4/8) symmetry in the spontaneous compactification of $D=11$ supergravity, Phys. Lett. 121B (1983) 141-146, http://dx.doi.org/10.1016/0370-2693(83)90903-6.

[257] D. Montgomery, H. Samelson, Transformation groups of spheres, Ann. of Math. (2) 44 (1943) 454-470.

[258] J. Simons, On the transitivity of holonomy systems, Ann. of Math. (2) 76 (1962) 213-234.

[259] S.W. Beck, J.B. Gutowski, G. Papadopoulos, AdS 5 backgrounds with 24 supersymmetries, J. High Energy Phys. 06 (2016) 126, http://dx.doi.org/10. 1007/JHEP06(2016)126, arXiv:1601.06645.

[260] A.S. Haupt, S. Lautz, G. Papadopoulos, $\mathrm{AdS}_{4}$ backgrounds with $\mathrm{N}>16$ supersymmetries in 10 and 11 dimensions, J. High Energy Phys. 01 (2018) 087 , http://dx.doi.org/10.1007/JHEP01(2018)087, arXiv:1711.08280

[261] A.S. Haupt, S. Lautz, G. Papadopoulos, A non-existence theorem for $N>16$ supersymmetric $\mathrm{AdS}_{3}$ backgrounds, 2018, arXiv: 1803.08428.

[262] B.E.W. Nilsson, C.N. Pope, Hopf fibration of eleven-dimensional supergravity, Classical Quantum Gravity 1 (1984) 499, 499 (1984), http://dx.doi.org/ $10.1088 / 0264-9381 / 1 / 5 / 005$.

[263] S. Klaus, Einfachzusammenh angende kompakte homogene Raume bis zur Dimension 9 (Diploma thesis), University of Mainz, 1988.

[264] S.G. Nikonorov, Compact homogeneous Einstein 7-manifolds, Geom. Dedicata 109 (2004) 7, http://dx.doi.org/10.1007/s 10711-004-3559-4.

[265] U. Gran, J. Gutowski, G. Papadopoulos, All superalgebras for warped $\mathrm{AdS}_{2}$ and black hole near horizon geometries, 2017, arXiv:1712.07889.

[266] H.B. Lawson, M.L. Michelsohn, Spin Geometry, Princeton University Press, 1998,

[267] F.R. Harvey, Spinors and Calibrations, Academic Press, 1990,

[268] M.Y. Wang, Parallel spinors and parallel forms, Ann. Global Anal. Geom. 7 (1989) 59

[269] S. Kobayashi, K. Nomizu, Foundations of Differential Geometry, vol. 2, Wiley Classics Library Edition, USA, 1996,

[270] L. Brink, P.S. Howe, Eleven-dimensional supergravity on the mass-shell in superspace, Phys. Lett. 91B (1980) 384-386, http://dx.doi.org/10.1016/ 0370-2693(80)91002-3. 